\title{
Probing the interior physics of stars through asteroseismology
}

\author{
C. Aerts $\mathbb{0}^{*}$ \\ Institute of Astronomy, Department of Physics and Astronomy, KU Leuven, \\ Celestijnenlaan 200 D, 3001 Leuven, Belgium
}

\section{(published 21 January 2021)}

\begin{abstract}
Yearslong time series of high-precision brightness measurements have been assembled for thousands of years with telescopes operating in space. Such data have allowed astronomers to measure the physics of stellar interiors via nonradial oscillations, opening a new avenue to study the stars in the Universe. Asteroseismology, the interpretation of the characteristics of oscillation modes in terms of the physical properties of the stellar interior, brought entirely new insights in how stars rotate and how they build up their chemistry throughout their evolution. Data-driven space asteroseismology has delivered a drastic increase in the reliability of computer models mimicking the evolution of stars born with a variety of masses and metallicities. Such models are critical ingredients for modern physics as a whole because they are used throughout various contemporary and multidisciplinary research fields in space science, including the search for life outside the Solar System, archaeological studies of the Milky Way, and the study of single and binary supernova progenitors, among which are future gravitational wave sources. The specific role and potential of asteroseismology for those modern research fields are illustrated. The review concludes with current limitations of asteroseismology and highlights how they can be overcome with ongoing and future large infrastructures for survey astronomy combined with new theoretical research in the era of high-performance computing. This review presents results obtained through major community efforts over the past decade. These breakthroughs were achieved in a collaborative and inclusive spirit that is characteristic of the asteroseismology community. The review's aim is to make this research field accessible to graduate students and readers coming from other fields of physics, with incentives to enjoy and join future applications in this domain of astrophysics.
\end{abstract}

DOI: 10.1103/RevModPhys.93.015001

\section{CONTENTS}

I. Looking Deep into Stars

A. Stars and their "good" vibrations

B. The beginnings of space asteroseismology

C. Asteroseismology to improve stellar evolution

D. Working in the Fourier domain truly helps

1. Mode damping and mode lifetimes

2. Undamped oscillations with quasi-infinite lifetimes

3. Damped oscillations with short lifetimes

II. Nonradial Oscillations of Stars

A. Stars and their hydrodynamics

1. The stellar structure equations

2. Simplification to 1D stellar models

3. Standard 1D stellar models in hydrostatic equilibrium

4. Nonstandard 1D models with microscopic atomic diffusion

5. Nonstandard 1D models with rotation and waves

6. One-dimensional equilibrium models as input for asteroseismology

B. Linear nonradial oscillation modes

1. Pressure and gravity modes

*Also at Department of Astrophysics, IMAPP, Radboud University Nijmegen, Heyendaalseweg 135, 6525 AJ Nijmegen, Netherlands and Max Planck Institute for Astronomy, Koenigstuhl 17, 69117 Heidelberg, Germany.

Conny.Aerts@kuleuven.be; https://fys.kuleuven.be/ster/staff/connyaerts
2. Asymptotic representations of high-order modes 25

3. Rotational splitting in a perturbative approach 28

4. Gravitoinertial modes in the traditional approximation

5. Rossby modes

III. Principles of Asteroseismic Modeling

A. Excitation mechanisms 31

1. Heat mechanisms and stochastic driving 31

2. Nonlinear resonant mode excitation 32

3. Convectively driven internal gravity waves 33

4. Tidal excitation of nonradial modes 34

B. Mode identification 36

1. Mode identification from échelle diagrams 36

2. Mode identification from rotationally split multiplets

3. Mode identification from period-spacing patterns 36

C. Asteroseismic modeling using mode frequencies $\quad 37$

1. Some modeling preliminaries 37

2. Setup of the modeling approach 38

3. Considering individual stars and ensembles $\quad 39$

IV. Applications of Asteroseismic Modeling 40

A. Sizing, weighing, and aging stars with convective envelopes

B. Assessing sharp features in stellar structure 43

C. Improving the physics of cool-star surface convection 45

D. Improving the theory of angular momentum transport 46

E. Inference of internal mixing from $g$ modes 48

F. The beginnings of tidal asteroseismology 49

V. Road Map for the Future 51

Acknowledgments $\quad 52$

References $\quad 52$ 


\section{LOOKING DEEP INTO STARS}

In his "News and Views" published in the journal Nature, Gough (1985b) announced the "beginnings of asteroseismology." "He explained that this is "the science of determining the internal structure of stars from the properties of dynamical oscillations," following the earlier introduction of the term in the scientific community by Christensen-Dalsgaard (1984) during a conference in Meudon. Gough ends his Nature article with the exciting prospect of "getting direct information about the stratification of the energy-generating core of a distant star," which sounded like a revolutionary idea at the time. A decade later, a less optimistic view was expressed by Brown and Gilliland (1994) in the introduction of their review: "The Sun is (and will likely remain) the outstanding example of the progress that can be made using seismological methods."

It is remarkable that a cosmologist was more optimistic on the matter than the experts, as expressed by Longair (2001) in his invited reflection: "At the same time, we need to understand the internal structures of the stars. In 1915, the breakthrough came with the plotting of the Hertzsprung-Russell diagram for a few hundred stars for which distances had been measured. The counterpart for the 21 st century will be asteroseismology, the direct measurement of the internal structure of the stars by measuring their normal modes of oscillation. It is salutary to note that helioseismology has revolutionized our understanding of the interior of the Sun in ways which could not necessarily have been predicted. The precise location of the boundary between the radiation- and convection-dominated zones and their three-dimensional structures are spectacular advances-a major goal of the astronomy of the future must be to perform the same studies on the stars present in the Hipparcos Hertzsprung-Russell diagram."

Meanwhile, we have Gaia Hertzsprung-Russell diagrams (HRDs) based on space astrometry with microarcsecond precision for more than $10^{9}$ stars in the Milky Way and beyond (Babusiaux et al., 2018), and asteroseismology for tens of thousands of those. Indeed, the past decade has seen the assembly of long-duration (up to four uninterrupted years) photometric data thanks to dedicated space missions, leading to the time-variable properties of stars derived with precisions of micromagnitude ( $\mu \mathrm{mag})$. This corresponds to flux variations at levels of parts per million ( $\mathrm{ppm}$ ). The primary research goal of several of those missions was the search for exoplanets around distant stars, but this is fine: the machinery delivered the data appropriate for asteroseismology (quite often, derogatorily, called "stellar noise" by exoplanetologists, while it actually concerns beautiful stellar signals; see the later discussion of Fig. 3. Asteroseismology based on these space photometric light curves meanwhile delivered interior rotation rates, stratification properties, and ages of thousands of distant stars, with impressive relative precisions unachievable by other methods; see Aerts, Mathis, and Rogers (2019), Table 1 . The past few years, we have even reached the status of

\footnotetext{
${ }^{1}$ For the etymology of this specific term in astrophysics, see Gough (1996) in response to the anecdote on the terminology raised by Trimble and Leonard (1996).
}

being able to derive the core rotation frequency for more than a thousand stars and the near-core mixing for many of them. The Sun is no longer the outstanding example to assess the properties of the deep interiors of stars. Those properties have become accessible thanks to the detection and interpretation of hundreds of nonradial oscillations with probing capacity of the deepest internal layers of the stars. Such modes have now been identified in stars across almost the entire stellar mass range and have turned stellar interiors into observational territory.

History has shown the optimistic daring views to be visionary and at the same time entirely justified. The study of stellar interiors is currently a data-driven modern topic. As we highlight in this review, asteroseismology not only reveals the need for better models of stellar structure and evolution but is paving the way toward them. It brings us into the renaissance of stellar evolution theory and does so from a multidisciplinary approach relying on space science and technology coupled with mathematical modeling. More particularly, asteroseismology involves data analysis methods such as time-series analysis, pattern recognition, and statistical modeling, while relying on various fields of physics and chemistry such as thermodynamics, nuclear and atomic physics, and quantum mechanics. The bridging of these scientific fields, starting from the appropriate observational input, allows us to achieve the long-awaited calibration of the physical properties of stellar interiors. We anticipate that the asteroseismically calibrated stellar models will be highly beneficial for various fields of research in astronomy, and in computational physics in general.

We now provide concise discussions of some key observational and data analysis aspects of asteroseismology, omitting many of the details, which can be found in the referenced literature. The bulk of the review then focuses on calibrating and improving the physics of stellar interiors, much in line with the previous quotations and with the purpose of Reviews of Modern Physics.

\section{A. Stars and their "good" vibrations}

Stellar variability is omnipresent in the HRD, which is a key diagnostic diagram used to evaluate stellar evolution theory. Such evaluations are often done by comparing the position of observed stars in this diagram with evolutionary tracks, such as the ones indicated by the full lines in Fig. 1. These tracks are based on particular versions of stellar evolution theories, of which there are many variants, as further outlined in Sec. II.A. Those types of comparisons between observations and theory are merely a crude evaluation because the HRD relies on only two quantities: the effective temperature of the star $T_{\text {eff }}$ and its luminosity $L$ (usually expressed in solar luminosity $L_{\odot}$ ). As detailed in Sec. II.A, stellar models contain a multitude of free parameters and rely on input physics suffering from uncertainties. The evaluation of these models therefore requires additional observational diagnostics to accompany the position of an observed star in the HRD. Surface abundances derived from high-precision spectroscopy offer important constraints in this respect, among various other observational diagnostics of the stellar atmosphere. These typically reach 


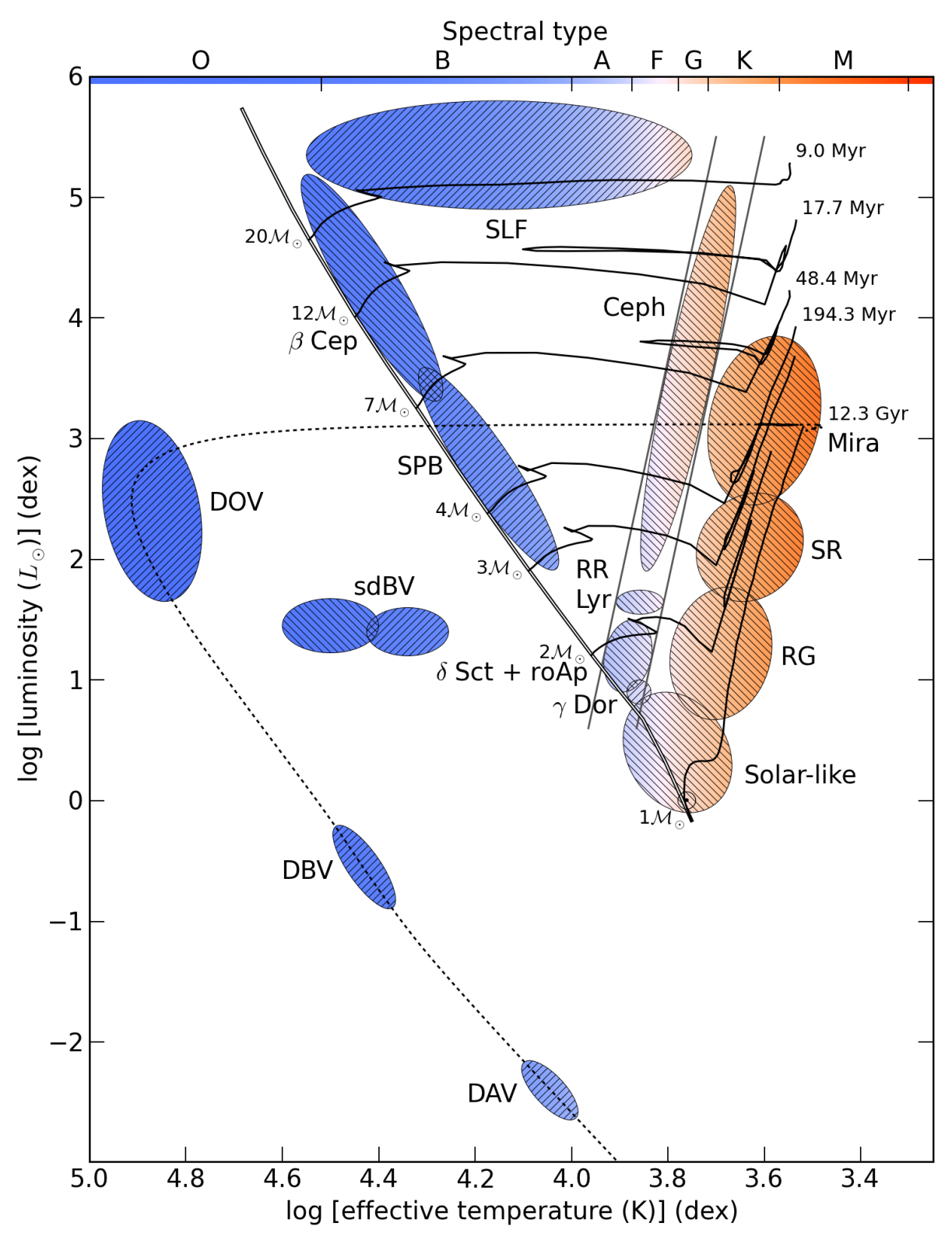

FIG. 1. Hertzsprung-Russel diagram (HRD) showing the position of different classes of pulsating stars. The abbreviation of the classes follows the nomenclature used by Aerts, Christensen-Dalsgaard, and Kurtz (2010) in Chap. 2, to which we refer for extensive discussions of all indicated classes in terms of the excitation mechanisms, along with the typical periods and amplitudes of the oscillations. The hatching line style used in each of the ellipses marks the dominant type of oscillation mode in each class: // for gravity modes and $\backslash \backslash$ for pressure modes. The recently discovered stochastic low-frequency (SLF) variability in O-type stars and blue supergiants is discussed in the text and has been added as a compararison with previous versions of this plot. The solid black lines and the black dotted line represent standard evolutionary model tracks, with birth masses and evolutionary timescales as indicated. The borders of the classical instability strip are plotted with gray lines, while the double line represents the zero-age main sequence (ZAMS). Early versions of this figure were made by Jørgen Christensen-Dalsgaard (Aarhus University) and Pieter Degroote (KU Leuven). Adapted from Pápics, 2013.

relative precisions of $1 \%$ to $5 \%$ for the best cases; see Table 1 given by Aerts, Mathis, and Rogers (2019).

A new view on stellar variability in the HRD is offered by data from the European Space Agency (ESA) Gaia satellite (Eyer et al., 2019). Using 22 months of calibrated photometric, spectrophotometric, and astrometric Gaia data, this study showed how the large-amplitude radial modes of classical variables, such as Cepheids, RR Lyrae stars, and Miras (indicated in Fig. 1), makes them "move" in the observational analog of the HRD, i.e., a color-absolute magnitude diagram, during their pulsation cycle. This introduces a new "time" dimension in the evaluation of stellar evolution theory. These radial pulsators remain of vast interest and importance for observational cosmology (Soszyński et al., 2016; Anderson and Riess, 2018) but are not considered in this review. Our attention is directed entirely to stars exhibiting multiple nonradial oscillations, which in the context of asteroseismology deserve to be called "good vibrations" after the eponymous 1966 song by the Beach Boys. 
From a physical viewpoint, nonradial oscillation modes are solutions to the equation of motion of a star that gets perturbed from its equilibrium. The modes are classified into two main groups according to which of the two forces, the pressure force or the buoyancy force of Archimedes, is dominant in restoring the equilibrium. Modes dominantly restored by the pressure force are called pressure modes, or "p modes" for short. These mainly have large amplitude in the envelopes of stars and are characterized by dominant radial motions. Gravity modes, or "g modes," are dominantly restored by the buoyancy force of Archimedes and attain large amplitudes in the deep interior of the star; they are characterized by dominant horizontal motions. As stars evolve, a powerful type of mode having a pressuremode character in the envelope and a gravity-mode character in the deep interior emerges. These so-called mixed modes have excellent probing power throughout the entire star.

A formal mathematical definition of nonradial oscillation modes is given in Sec. II. However, it is instructive to already know what the modes look like. One can consider nonradial modes of a 3D spherical star as the analogy of the vibration modes of a 1D string. Each vibration mode of a string makes it deviate from its equilibrium position and is characterized by three numbers: its frequency, its amplitude, and its number of nodes $n$. The nodes are points where the string does not move during the vibration cycle. One adopts the terminology that $n=0$ corresponds to the fundamental vibration mode of the string: $n=1$ to the first overtone, $n=2$ to the second overtone, etc. Each nonradial mode of a 3D star makes the gas particles in this star deviate from their equilibrium position and is also characterized by a frequency and an amplitude, but now three integer numbers are needed to indicate the positions of the nodes of the displacement vector with respect to a symmetry axis of the star. Given that it concerns a 3D spherically symmetric body whose fluid elements get displaced from their equilibrium position by a vector $\xi=\left(\xi_{r}, \xi_{\theta}, \xi_{\phi}\right)$, the angular geometry of this vector is described in terms of a spherical harmonic function, containing a Legendre polynomial $P_{l}^{m}$ as a function of colatitude $\theta$ and a harmonic function in terms of azimuth $\phi$. The rotation axis is usually taken as the symmetry axis of the modes. Thus, for each nonradial oscillation mode, three labels $(l, m, n)$ are used to indicate the nodes of the mode, where $l$ is the total number of nodal lines on the stellar surface and $|m|$ of those nodal lines pass through the symmetry axis. The $n$ value again indicates the overtone of the mode, which now concerns the number of nodal shells situated inside the star that do not move during the oscillation cycle. The special case of a radial mode has $l=m=0$ and displaces the fluid elements inside the star in the radial direction only.

The symmetry axis of the oscillations is "inclined" with the line of sight of a distant observer by an unknown angle called the inclination angle $i$. Figure 2 gives a visual representation of the radial component of the displacement vector $\xi_{r}$ for some typical nonradial modes "observed" under an inclination angle of $60^{\circ}$. The term observed is a bit misleading here because stellar surfaces cannot be resolved well enough to study the majority of nonradial oscillations of stars, except for the Sun. Rather, the signatures of the oscillation modes are "detected" in observables that are stellar quantities integrated over the part of the stellar disk that is visible for an observer.

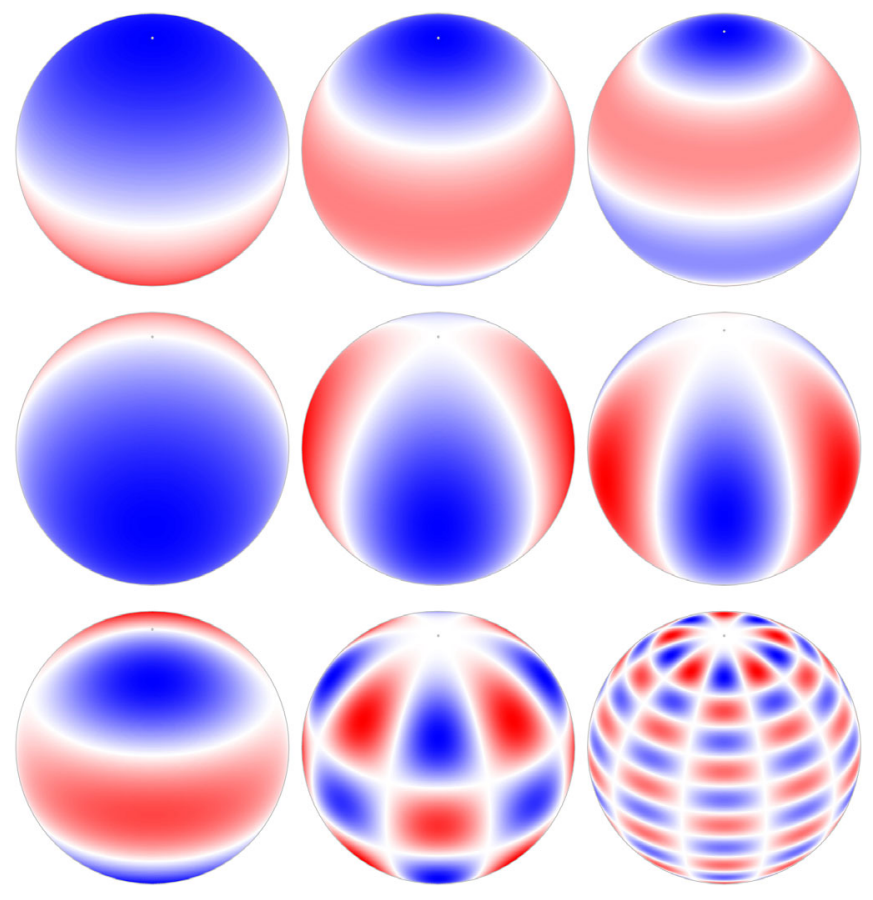

FIG. 2. Snapshot of the angular dependence of the radial component of the displacement vector $\xi_{r}$ at one point in the oscillation cycle for various nonradial modes, seen under an inclination angle of $60^{\circ}$. White bands indicate the positions where $\xi_{r}=0$; red and blue represent areas at the stellar surface moving in (out) at the chosen time. Shown from left to right are first row, axisymmetric $(m=0)$ modes with $l=1,2,3$; second row, sectoral $(l=|m|)$ modes with $l=1,2,3$; third row, tesseral $(l \neq|m|)$ modes with $(l,|m|)=(3,1),(6,4),(15,5)$. High-degree modes such as the two in the third row are usually not detected in space photometry due to cancellation effects when integrating the flux variations across the visible stellar disk.

The nonradial oscillations make some parts of the star move up (indicated in blue in Fig. 2), while others are going down (red patches in Fig. 2) periodically according to the eigenfrequency of the mode. Such motions imply small local changes in the velocity, temperature, and radius of the stellar gas, creating local flux variations. These flux variations change periodically in time during the oscillation cycle, i.e., half a cycle further the red patches in Fig. 2 will have become blue, and vice versa. The surface-integrated effect due to each nonradial mode measured in flux or velocity variations by an observer depends on the inclination angle because it is determined by the position of the surface nodal lines in the line of sight. This interplay between the geometry of the mode and the value of $i$ gives rise to so-called partial cancellation due to integration over the visible stellar disk, which increases as the degree of the mode increases; see Figs. 1.4 and 1.5 given by Aerts, Christensen-Dalsgaard, and Kurtz (2010). In particular, when nonradial modes are seen under their angle of complete cancellation, they do not lead to variability, while the latter is maximal when seen under their optimal angle of least cancellation. For the values of these special mode angles, we refer to Table B.1 in Appendix B of Aerts, ChristensenDalsgaard, and Kurtz (2010). It is also noteworthy that partial cancellation works differently in photometric versus 
spectroscopic data, because the integrated flux is highly sensitive to limb darkening but the integrated velocity is less sensitive to it. In addition, the effect is different for $\mathrm{p}$ and $\mathrm{g}$ modes. The sensitivity to limb darkening is smaller for $\mathrm{p}$ modes because their $\xi_{r}$ is dominant in the line of sight, while $\mathrm{g}$ modes have dominant $\xi_{\theta}$ and $\xi_{\phi}$ and are hence much more prone to limb-darkening effects for an observer.

Measuring the small flux or velocity variations during an oscillation-mode cycle allows us to derive the mode's period without having to resolve the stellar surface. This is how the time-variability aspect of asteroseismology works. It is in principle an easy aspect of the research provided that one has data with a high duty cycle, which is defined as the fraction of the mode period covered with data expressed as a percentage. In practice, the overall beating cycle encapsulating the global pattern due to all active modes of the star has to be covered with a high duty cycle. Moreover, the data need to have noise levels below the amplitudes of the modes in the appropriate frequency regime. These scientific requirements become easier to meet the longer the time series and the more data points that one has available. Detecting oscillation mode frequencies and estimating their uncertainty is also much easier to do from uninterrupted data with a high duty cycle than from gapped time-series data with a low duty cycle.

Ledoux (1951) proposed the occurrence of two nonradial $p$ modes in a rotating star as the explanation for the detected variable velocity behavior of the star $\beta$ Canis Majoris. His landmark paper provided the first correct interpretation and understanding of the observed biperiodic variability (i.e., caused by two simultaneously active $\mathrm{p}$ modes) of a rotating star in terms of the physics of nonradial oscillations. As a member of the class of $\beta$ Cep stars, $\beta$ Canis Majoris was thus the first star with confirmed nonradial modes occupying the proper ellipse in Fig. 1. It took another 41 years until the excitation mechanism of those nonradial oscillations was understood in terms of a heat mechanism, also known as the opacity mechanism (Moskalik and Dziembowski, 1992). We return to mode excitation mechanisms in Sec. III.A.

During the half century following Ledoux's insightful 1951 paper, the search for and identification of nonradial oscillations in time-series observations became an active research field. Inventories of nonradial modes and their identification in terms of the spherical wave numbers $l$ and $m$ (see Sec. II and Fig. 2) grew steadily. Nevertheless, asteroseismology in the spirit of Gough and Longair, i.e., with the aim to improve the interior physics of stars undergoing nuclear fusion, was nowhere near the horizon. Major successes were, however, booked for $\mathrm{g}$ modes of white dwarfs along their cooling track in the HRD; see Fig. 1. Brown and Gilliland (1994) indeed discussed the category of the stars "unlike the Sun." They illustrated that white-dwarf asteroseismology based on weekslong ground-based multisite monitoring of nonradial oscillations was furthest advanced, and that the next best cases of the rapidly oscillating Ap (roAp) and $\delta$ Sct stars (see Fig. 1) were still limited in terms of physical interpretation. For none of the other classes of nonradial pulsators in Fig. 1 did one come anywhere near making inferences on how to improve the physics of their interiors from exploitation of the available detected nonradial oscillations.
Although major achievements were obtained in the decade after this first review on asteroseismology by Brown and Gilliland (1994), mainly from ground-based multisite network campaigns for pulsating white dwarfs (Winget et al., 1991), hot subdwarfs (Kilkenny et al., 1999; Brassard et al., 2001), roAp stars (Kurtz et al., 2005), $\delta$ Sct stars (Breger et al., 2005), and $\beta$ Cep stars (Handler et al., 2006), the plea by Brown and Gilliland (1994) to replace photometric ground-based network observations with data to be taken with spaceborne telescopes was fully justified. Space data not only would provide much lower noise by avoiding disturbances due to Earth's atmospheric variability but also would allow one to increase the duty cycles of the data significantly, without large daily interruptions of the time series that plague data from ground-based observatories. Indeed, even successful multisite campaigns remained below $50 \%$ duty cycle, meaning that the oscillation cycles were never covered appropriately, except for white dwarfs, subdwarfs, and roAp stars, all of whose oscillations have periods of only a few to tens of minutes and dominant mode amplitudes of the order of millimagnitudes, corresponding with levels of parts per thousand (ppt) when considering the star's flux variability rather than its change in brightness expressed in magnitude. In retrospect, the gain from space photometry was illustrated by Zwintz et al. (2000), who analyzed ten years of Fine Guidance Sensors photometry of tens of thousands of supposedly constant guide stars observed with the Hubble Space Telescope to stabilize the satellite. They found variability in about 20 stars, among them four $\mathrm{K}$ giants revealing periods of a few hours. They reported this to be incompatible with rotational variability but did not interpret it in terms of oscillations, which we now know are the cause.

While awaiting space photometry, the hunt for solarlike oscillations in solar twins from radial-velocity time series grew fast after the predictions published in the seminal paper by Kjeldsen and Bedding (1995). The ever increasing precision reached by spectrographs led to the first firm discoveries of individual solarlike oscillation modes in the nearby Sun-like stars $\eta$ Boo (Kjeldsen et al., 1995), $\beta$ Hyi (Bedding et al., 2001), and $\alpha$ Cen A (Bouchy and Carrier, 2001), after earlier unconfirmed attempts to find them in Procyon (Brown et al., 1991). Oscillation modes were also detected in radialvelocity variations of the red giant $\xi$ Hya (Frandsen et al., 2002). By the time of space asteroseismology, detections of solarlike oscillations had been achieved for about 25 bright stars; see Figs. 2.2 and 2.3 given by Aerts, ChristensenDalsgaard, and Kurtz (2010) for summary plots. All those spectroscopic data revealed mode frequencies as expected from scaling those of the Sun, which was in line with the predictions made by Kjeldsen and Bedding (1995).

\section{B. The beginnings of space asteroseismology}

A half century of intense monitoring of pulsators with nonradial oscillation modes from ground-based observatories since Ledoux's 1951 analysis took place. Despite heroic achievements in terms of number of detected nonradial mode frequencies in $\delta$ Sct stars [as summarized byBreger (2000), its pioneer], the struggle with daily alias frequencies due to periodic gaps in the data and the lack of unambiguous identification of their $(l, m)$ could hardly be overcome. 
This disappointing situation got placed in a new light thanks to an opportunity that occurred by accident, and that is to be taken literally. The NASA Wide Field Infra Red Explorer (WIRE) lost its coolant after launch and could not perform the science it was designed for. Buzasi convinced NASA to reorient the WIRE satellite project into a proof-of-concept asteroseismology mission by using its onboard $5 \mathrm{~cm}$ tracker telescope and camera to monitor the variability of various kinds of bright stars uninterruptedly and with high cadence during several weeks. Despite major instrumental effects due to telescope jitter (the machinery was absolutely not built to do what it was used for), this blessing in disguise immediately showed the potential gain that could be achieved should a dedicated specifically designed asteroseismology space mission become available: Buzasi (2000) discovered oscillation modes in the red giant $\alpha \mathrm{UMa}$, as anticipated by Brown and Gilliland (1994). Despite having been an unplanned pioneer, WIRE achieved ppt-level amplitude detections and above all illustrated the great improvement of being able to observe uninterruptedly from space. Among other results, it led to detections of oscillations in $\mathrm{K}$ giants (Stello et al., 2008), showed nonradial modes to be present in the bright $\delta$ Sct star Altair (Buzasi et al., 2005), where ground-based monitoring had failed to find any, and drastically improved light curves of eclipsing binaries (Southworth, Bruntt, and Buzasi, 2007).

Canada's first space mission, Microvariability and Oscillations of Stars (MOST, launched in 2003) (Walker et al., 2003), was also the first space mission dedicated to space asteroseismology, although it observed all sorts of stellar variability. Given its modest aperture of $15 \mathrm{~cm}$, it is known in the asteroseismology community as the HST ("Humble Space Telescope") baptized as such by its principal investigator (PI) Jaymie Matthews. MOST data revealed numerous oscillation modes in stars belonging to almost all classes indicated in Fig. 1, such as red giants (Barban et al., 2007), roAp stars (Huber et al., 2008), $\delta$ Sct and $\gamma$ Dor stars (Rowe et al., 2006; Sódor et al., 2014), emission-line OB stars (Walker et al., 2005a, 2005b; Saio et al., 2007), isolated and cluster slowly pulsating B stars (SPBs) (Aerts et al., 2006; Cameron et al., 2008; Gruber et al., 2012), pre-main-sequence (pre-MS) stars (Zwintz, 2008, 2009), and many more [see Matthews (2007) for an early status report]. Even though it could monitor stars for a period of only about six weeks maximally (limiting the precision of the oscillation frequencies) and its photometric precision in the time domain was of the order of ppt, it revealed many more oscillation modes than what had been achieved from ground-based campaigns. In many respects MOST was a highly successful (and inexpensive) planned pioneering mission.

The first "major" space mission dedicated to the monitoring of numerous nonradial pulsators with the aim of space asteroseismology (along with exoplanet hunting) was the French-led CoRoT mission. It was launched in 2006 into a low-Earth orbit and was operational until 2012 (Auvergne et al., 2009; Baglin et al., 2009). Its original acronym CoRot stood for "convection and rotation," but later in the project the exoplanet hunting was added to get the mission funded and hence the "t" was changed to uppercase to represent "convection, rotation, and exoplanetary transits." CoRoT carried a $27 \mathrm{~cm}$ telescope and was dedicated to asteroseismology of tens of bright stars ( $V$ magnitude between 5 and 9) monitored with a cadence of $32 \mathrm{~s}$ and exoplanet hunting around thousands of faint stars ( $V$ magnitude between 11 and 16) measured every $15 \mathrm{~min}$ during each of its pointings; see Auvergne et al. (2009) for the technical and operational details pertaining to the mission. Because of its construction and lowEarth orbit, CoRoT was able to point in the center or anticenter direction of the Milky Way over five uninterrupted months (its so-called long runs), between which it did short runs of about a month in duration. This meant that the target selection and the choice of the fields of view (FOVs) to point at were critical and had to be optimized to meet the wishes of two until then hardly collaborating communities, the asteroseismologists and the exoplanet hunters, from the numerous countries that funded the mission. This "astrosociological" aspect of the mission led to heated debates (in various languages) during the so-called CoRoT weeks, which were preparatory workshops held twice a year to optimize the mission planning and exploitation. Had it not been for the heroic leadership of the mission PI Annie Baglin, we would have kept on changing our minds about the pointings until the day of the launch.

CoRoT was a major success on various fronts. It properly allowed asteroseismology of Sun-like stars, as done by Appourchaux et al. (2008), Michel et al. (2008), Benomar et al. (2009), García et al. (2009), Deheuvels et al. (2010), Mathur et al. (2010), and Ballot et al. (2011), where the last study treated an exoplanet host star. It also led to the discovery of nonradial oscillations in red giants (De Ridder et al., 2009), opening up the major unexploited parameter space of socalled solarlike oscillations in evolved stars, as studied by Barban et al. (2009), Hekker et al. (2009), Miglio et al. (2009), Kallinger et al. (2010), and Mosser et al. (2010). Applications to other types of nonradial pulsators are too numerous to mention, but a few breakthroughs were the discovery of outbursts with accompanying mass loss in $\mathrm{Be}$ stars due to the nonlinear interaction between nonradial modes observed in real time (Huat et al., 2009), the occurrence of stochastic nonradial oscillations in B-type stars [see Belkacem et al. (2009), Degroote et al. (2010b), including in the gravitoinertial regime, Neiner et al. (2012); explanation of these types of modes is given later], asteroseismic modeling of an O9 star (Briquet et al., 2011), the discovery of lowfrequency variability in O-type stars that remained unexplained at that time [see Blomme et al. (2011); interpretations are given later], and several eclipsing binaries with tidally induced or tidally affected nonradial oscillations, such as given by Maceroni et al. $(2009,2013)$ and da Silva et al. (2014). Many other results remain unmentioned here. A special volume of the journal Astronomy and Astrophysics ${ }^{2}$ was dedicated to 55 CoRoT papers and offers the reader an extensive review on the mission's instrument performance and scientific results.

And then came the successful NASA Kepler mission (Koch et al., 2010), launched in 2009, delivering light curves of unprecedented quality, as shown in Fig. 3 for a few stars observed in its long-cadence mode (29.43 min). Kepler

\footnotetext{
${ }^{2}$ Journal volume available in open access at https://www.aanda .org/component/toc/?task=topic\&id=9.
} 


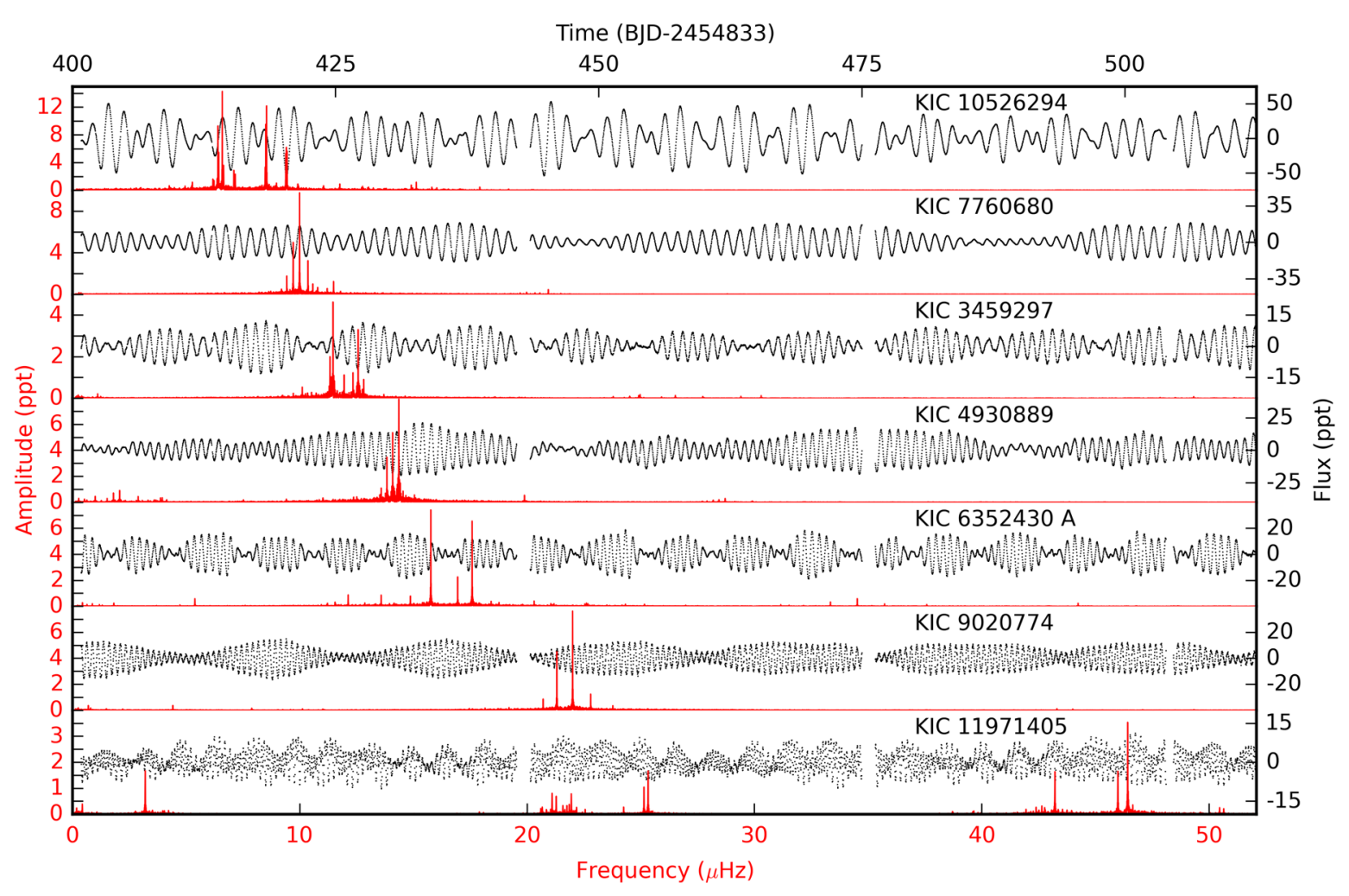

FIG. 3. Excerpts of $110 \mathrm{~d}$ duration (of the total $\sim 1500 \mathrm{~d}$ ) extracted from the Kepler long-cadence ( $\sim 30$ min per point) light curves (in black dots) of seven slowly pulsating B stars (cf. Fig. 1) indicated with their Kepler input catalog identification (KIC) (Brown et al., 2011). The amplitude spectrum obtained from a Fourier transform of the full Kepler light curves is overplotted in red. These stars exhibit nonradial gravity modes with individual mode periods of the order of $1 \mathrm{~d}$. The light curves reveal a gallery of diverse beating patterns among the modes and a gradual shift in maximum amplitude from low to high frequency as their rotation frequency changes from low in the top panel to high in the bottom panel. Despite their large amplitudes of several to $12 \mathrm{ppt}$, none of these stars were known to have nonradial oscillations prior to the Kepler mission; it is notoriously difficult to detect modes with such periodicities from ground-based data given their similarity with the rotation period of Earth. Adapted from Pápics et al., 2017.

delivered light curves with a duration of four years, for about 200000 low- and intermediate-mass stars in one FOV in the northern sky. These data have a 10 times longer time base and deliver a factor $\sim 100$ better precision for the oscillation frequencies than the CoRoT data, thanks to the larger aperture of the telescope $(0.95 \mathrm{~m})$, the longer pointing, and the more stable Earth-trailing orbit. The nominal Kepler mission lasted four years and had a dedicated asteroseismology program (Gilliland et al., 2010), monitoring several hundred low-mass stars at a short cadence of $58.85 \mathrm{~s}$. After the nominal four-year mission, the Kepler spacecraft lost two of its four working reaction wheels. The mission was then repurposed as a space project monitoring fields in the ecliptic, making clever use of the solar radiation pressure to stabilize the satellite. This mission operated under the name K2 and monitored 19 fields, each of which during maximally $\sim 80 \mathrm{~d}$ between February 2014 and October 2018, adopting the same cadence types as Kepler (Howell et al., 2014). Because of its superior quality, most of the results discussed in this review are based on Kepler (or K2) data, so we do not summarize results here as we did for the other space missions.

The Bright Target Explorer constellation (BRITE; launched in 2014 and currently operational) is a set of Austrian, Canadian, and Polish nanosatellites assembling multicolor photometry of the brightest stars in the sky for variability studies and asteroseismology (Weiss et al., 2014). BRITE is unique in that it offers two-band photometry based on a narrow blue and a broad red filter. While the data reduction was initially a challenge, given the limited weight and pointing stability of the small satellites, its photometric precision currently reaches ppt per data point. It can monitor selected stars during about half the year (Pablo et al., 2016). BRITE is monitoring a variety of bright variables. Its data of high-mass stars is complementary to the Kepler data in terms of targets. BRITE revealed several more nonradial oscillation modes than what has been found in ground-based data for OB- and Be-type pulsators (Baade et al., 2016; Pigulski et al., 2016; Handler et al., 2017; Kallinger et al., 2017; Ramiaramanantsoa et al., 2018). Combined BRITE data and archival data assembled from extensive ground-based (multisite) campaigns or data that are currently being assembled by the NASA Transiting Exoplanet Survey Satellite (TESS) (Ricker et al., 2016) hold good potential for asteroseismology of the highest-mass nearby stars with highamplitude oscillation modes to perform modeling of their interior properties.

Handler et al. (2019) illustrated the TESS potential in revisiting bright B-type pulsators discovered from the ground but lacking sufficient identified pulsation modes. The nominal TESS mission is scanning almost the full sky, delivering highprecision space photometry for millions of stars with time 
bases between 27 and $352 \mathrm{~d}$ for each of the hemispheres, with similar cadences as Kepler. This difference in duration of the monitoring is due to its operational scheme of observing in sectors (13 per hemisphere), each monitored over $27 \mathrm{~d}$. Increasing partial overlap between the consecutive sectors occurs for areas in the sky closer to the ecliptic pole, with a maximum of continuous observation over $352 \mathrm{~d}$ for stars in the TESS continuous viewing zone (CVZ). TESS samplings changed to $10 \mathrm{~min}$ and $20 \mathrm{~s}$ for the long- and short-cadence modes, respectively, in the extended mission (started in mid 2020). Owing to the more limited time base it delivers far less precise oscillation frequencies for asteroseismology than Kepler, but it opens up the entire sky to provide large samples of pulsators. Some of these samples were not yet treated by CoRoT and Kepler, including metal-poor high-mass stars in the Large Magellanic Cloud (LMC). Thus far large asteroseismology samples have essentially been limited to redgiant pulsators. In that sense, TESS will bring major advances for a variety of stars across the HRD in Fig. 1, from low-mass unevolved Sun-like stars (Schofield et al., 2019), including exoplanet hosts (Campante et al., 2016b), all the way up to the most massive stars.

The past decade brought us to a golden era for asteroseismology, with the BRITE and TESS missions ongoing, the Planetary Transits and Oscillations of stars mission (PLATO) (Rauer et al., 2014) on the horizon, and an immense amount of Kepler data yet to be interpreted in the true meaning of asteroseismology, i.e., with the aim of improving the physics of stellar interiors. How to achieve that is discussed in the rest of the review.

\section{Asteroseismology to improve stellar evolution}

The HRD in Fig. 1 reveals that pulsational variables occupy many phases of stellar evolution. The periods of nonradial oscillations covered by stars and stellar remnants range from seconds to months, or even years, and their amplitudes of brightness variations cover the range of a magnitude to the current detection threshold of $\mu$ mag (corresponding to hundreds of ppt to ppm in flux variability). We refer to Table A.1 given by Aerts, Christensen-Dalsgaard, and Kurtz (2010) for a summary of the pulsation characteristics. The basic properties and the excitation mechanisms of all known classes of nonradial pulsators indicated in Fig. 1 were discussed in great detail in Chap. 2 given by Aerts, Christensen-Dalsgaard, and Kurtz (2010). Noteworthy discoveries of nonradial oscillations not yet firmly established from ground-based data were made for red giants from CoRoT (De Ridder et $a l ., 2009$ ) and for blue supergiants from MOST (Saio et al., 2006), Hipparcos (Lefever, Puls, and Aerts, 2007), and Kepler (Aerts et al., 2017). While oscillations were already discovered in red giants from ground-based spectroscopy, it was still heavily debated whether or not it concerned radial or nonradial oscillations (Frandsen et al., 2002). Both red giants and blue supergiants were established as nonradial pulsators from early Kepler (Bedding et al., 2010), K2 (Bowman et al., 2019b), and TESS data (Pedersen et al., 2019). Hence they received their own ellipse and Fig. 1 was adapted accordingly.

The Kepler spacecraft led to the discovery of more than 20 000 red giants with nonradial oscillations (Hon et al., 2019), and TESS will undoubtedly provide a factor of 10 more. Numbers for blue supergiants discovered from K2 and TESS are much lower, of the order of a few hundred, because these are rare objects. Moreover, they were omitted from the nominal Kepler FOV so as not to "disturb" the exoplanet hunting. The variability of O-type dwarfs and blue supergiants is caused by a complex interplay between various phenomena, which may involve internal gravity waves (IGWs) (Rogers et al., 2013), rotational modulation (Ramiaramanantsoa et al., 2018), subsurface convection (Grassitelli et al., 2015), wind variability (Krtička and Feldmeier, 2018), magnetism (Sundqvist et al., 2013), nonradial g modes (Moravveji, Moya, and Guinan, 2012), and binarity (Sana et al., 2012). The discovery of ubiquitous low-frequency power excess in hundreds of OB dwarfs and supergiants in the upper HRD from K2 and TESS space photometry by Bowman et al. (2019b) and Pedersen et al. (2019) granted them an ellipse in Fig. 1 labeled "SLF" for stochastic low-frequency variability.

Gautschy and Saio (1995, 1996) announced that the importance of nonradial oscillation studies would grow as monitoring capacities to detect ever smaller amplitude variability would improve: a visionary outlook a few years before space asteroseismology came about with WIRE. Figure 1 shows that stars across almost the entire mass range will encounter nonradial oscillations at particular stages of their evolution. The characteristics of these nonradial oscillation modes (their periods or frequencies, amplitudes, and mode lifetimes) offer great diagnostic value for inferences of the stellar interior. As outlined in detail in Sec. II, the mode frequencies or periods allow high-precision views of the physical properties inside stars that are not accessible by classical "snapshot-type" data assessing only the surface or atmospheric properties at a particular time of the variability cycle (such as single-epoch spectroscopy, color indices, or interferometry). The combination of high-cadence time-series data covering the overall pulsational variability cycle, along with observables representing the position in the HRD $\left(T_{\text {eff }}, L / L_{\odot}\right)$ and surface abundances from spectroscopy, constitute an optimal starting point for asteroseismic modeling. The power of combining such a classical and asteroseismic approach for stellar modeling was illustrated by Lebreton, Goupil, and Montalbán (2014) in their Fig. 13, with major improvement for stellar aging. In practice, the addition of asteroseismology to stellar modeling permits one to reach levels of $\sim 10 \%$ precision in stellar ages. Precise values for the stellar luminosity $L$ or the stellar radius $R_{\star}$ of low- and intermediate-mass stars can now be obtained from Gaia astrometry (Brown et al., 2018) and interferometry, respectively. Following the extensive review on how to bridge asteroseismology and interferometry by Cunha et al. (2007), this synergy turns out to be highly successful for the brightest pulsators with space asteroseismic data, as highlighted by North et al. (2007), Bazot et al. (2011), Huber et al. (2012), and White et al. (2013, 2018).

To conclude this section, nonradial oscillations occur all over the HRD. This offers the opportunity to perform asteroseismology for members of the classes indicated in Fig. 1 and to couple the conclusions into a coherent picture across stellar evolution. Such a metastudy has been done for 
asteroseismic estimates of the interior rotation of low- and intermediate-mass stars, leading to the conclusion that the theory of angular momentum transport in stellar interiors already needed improvement from the earliest stages of stellar evolution. We come back to this in Sec. III but stress here that a global perspective of stellar evolution across all life phases proved to be necessary to assess the weaknesses of one particularly important aspect of stellar evolution theory: the transport of angular momentum is much more efficient than predicted by theory; see Fig. 4 given by Aerts, Mathis, and Rogers (2019), which summarizes a global result based on major efforts by many scientists in the asteroseismology community. This is just one example of how asteroseismology paves the way toward better stellar evolution models. Data are now being assembled by the TESS mission to reach the same impact for high-mass stars. For the rest of this review, we adopt the same definition as Aerts, Mathis, and Rogers (2019) to discriminate between stars of low $\left(M_{\star} \lesssim 1.3 M_{\odot}\right)$, intermediate $\left(1.3 \lesssim M_{\star} \lesssim 8 M_{\odot}\right)$, and high $\left(M_{\star} \gtrsim 8 M_{\odot}\right)$ mass.

\section{Working in the Fourier domain truly helps}

Figure 3 is a textbook illustration of how stars of intermediate mass exhibiting $\mathrm{g}$ modes behave in flux as their interior rotation increases (from top to bottom in the plot). We explain in Sec. II how to arrive at such a conclusion about their interior rotation, but for now the message is that this result cannot be "seen" easily in the time domain (i.e., in the light curve in gray), while it can be directly distilled from the Fourier spectrum overplotted in red. It is impressive how much information about the stellar interior is contained in a Fourier transform of a light curve: the art is to get it out, and asteroseismologists are experts in this regard.

We see in Fig. 3 that the duty cycle of the Kepler data is high, but not $100 \%$, because the satellite had to be turned every three months to keep its solar panels pointed to the Sun. Moreover, one-month data downlink and momentum dump desaturation interruptions occurred approximately every $3 \mathrm{~d}$. Moreover, the Kepler photometer consisted of 21 CCD modules (Koch et al., 2010), but one of them (Module 3) broke down less than a year into the mission, causing gaps in the data for stars on that module. The Kepler spacecraft sampled the stars at a constant cadence, delivering data at precise time stamps. The data are modulated with $1-y r$ periodicity when transformed to the barycenter of the Solar System (Murphy, Shibahashi, and Kurtz, 2013). Given that stellar oscillations are periodic because they occur at the "eigenfrequencies" of the star, Fourier analysis offers an optimal frequency extraction method. It is extensively described in Chap. 5 given by Aerts, Christensen-Dalsgaard, and Kurtz (2010) in the general case of nonequidistant gapped time series of ground-based data. An excellent crash course on the topic was given by Appourchaux (2014), who tuned it to the modern era of space asteroseismology, while the effect of interruptions in the Kepler light curves on the frequency analysis for asteroseismology was thoroughly assessed by García et al. (2014). Basu and Chaplin (2017) provided even more detailed information and focused on data analysis in the case of solarlike oscillations. Here we limit ourselves to the bare minimum and pay specific attention to the aspects of time-series analysis from the viewpoint of having two major categories of nonradial oscillation modes: those that are damped and have short lifetimes and those that are undamped and, to a good approximation, have infinite lifetimes. These two cases require different data analysis approaches.

As explained later, the three components of the Lagrangian displacement vector due to a nonradial oscillation mode in the absence of rotation contain a common time-dependent factor $\exp (-\mathrm{i} \omega t)$, with $\omega=2 \pi \nu$ the angular frequency of the mode, $\nu$ its cyclic frequency, and $P=2 \pi / \omega=1 / \nu$ its period. In general, $\omega$ is an imaginary quantity $\omega=\omega_{r}+\mathrm{i} \omega_{i}$, but for the study of the periodic behavior of the mode to be derived from data we consider its real part (for simplicity denoted as $\omega$ in the rest of the review). Imagine that we want to extract the frequencies present in time-series data representing a continuous and finite function $x(t)$ (the flux variations in the case of space asteroseismology, indicated with the black dots in Fig. 3). The Fourier transform of $x(t)$ is given by

$$
F(\nu) \equiv \int_{-\infty}^{+\infty} x(t) \exp (2 \pi \mathrm{i} \nu t) d t
$$

By performing this transformation, we move from the time domain (black dots in Fig. 3) to the frequency domain (shown in red in Fig. 3). In the following case where $x(t)$ is a sum of harmonic functions with frequencies $\nu_{1}, \ldots, \nu_{M}$ and amplitudes $A_{1}, \ldots, A_{M}$ :

$$
x(t)=\sum_{k=1}^{M} A_{k} \exp \left(2 \pi \mathrm{i} \nu_{k} t\right)
$$

we find that

$$
F(\nu)=\sum_{k=1}^{M} A_{k} \delta\left(\nu-\nu_{k}\right)
$$

where $\delta$ is Dirac's delta function for which $\delta\left(\nu-\nu_{k}\right) \neq 0$ only for the frequencies $\pm \nu_{1}, \ldots, \pm \nu_{M}$. No matter how good the Kepler data are, the time series (1) contains discrete data points, (2) has finite duration, and (3) has gaps (even if they are small). This implies that one cannot compute the integral in Eq. (1). However, we can rely on the so-called window function defined by the data, measured at time points $t_{j}$, $j=1, \ldots, N$ during the time interval $[0, T]$, to obtain

$$
w_{N}(t) \equiv \frac{1}{N} \sum_{j=1}^{N} \delta\left(t-t_{j}\right)
$$

This allows us to write the discrete Fourier transform (DFT) of the function $x(t)$ as

$$
F_{N}(\nu)=N \int_{-\infty}^{+\infty} x(t) w_{N}(t) \exp (2 \pi \mathrm{i} \nu t) d t
$$

The following DFT of the window function is called the spectral window $W_{N}(\nu)$ : 


$$
W_{N}(\nu)=\frac{1}{N} \sum_{j=1}^{N} \exp \left(2 \pi \mathrm{i} \nu t_{j}\right)
$$

The DFT of the data is hence the convolution of its spectral window and its Fourier transform

$$
\begin{aligned}
F_{N}(\nu) & =N\left(F * W_{N}\right)(\nu) \\
& =\sum_{j=1}^{N} x\left(t_{j}\right) \exp \left(2 \pi \mathrm{i} \nu t_{j}\right) \Delta t_{j},
\end{aligned}
$$

where $\Delta t_{j} \equiv t_{j}-t_{j-1}$ (Deeming, 1975). Frequencies are often searched from the power density (PD) instead of the Fourier transform. The PD is defined as the power present in the signal as a function of frequency per unit frequency. Relying on Eq. (7), we get

$$
\mathrm{PD}(\nu) \equiv \frac{1}{T}\left|F_{N}(\nu)\right|^{2}
$$

The PD is hence expressed in $\mathrm{ppm}^{2} / \mu \mathrm{Hz}$ when the time series concerns flux measurements expressed in ppm and frequencies are expressed in microhertz.

For equidistant data, $t_{j}=j \Delta t$, where $\Delta t_{j}=\Delta t$ is a constant sampling interval between two consecutive measurements. In that case, we have

$$
\begin{gathered}
F_{N}(\nu)=\Delta t \sum_{j=1}^{N} x(j \Delta t) \exp [2 \pi \mathrm{i} \nu(j \Delta t)], \\
W_{N}(\nu)=\exp [\pi \mathrm{i} \nu \Delta t(N+1)] \frac{\sin (\pi \nu N \Delta t)}{N \sin (\pi \nu \Delta t)} .
\end{gathered}
$$

The Nyquist frequency of such data is half of the sampling rate $\nu_{\mathrm{Ny}}=1 / 2 \Delta t$. This frequency is defined as the upper limit of the frequency range over which the Fourier transform is unique. In principle this sets an upper limit for the interval of frequencies to search for. However, a particular frequency detected below $\nu_{\mathrm{Ny}}$ may be an alias of the true frequency that occurs above $\nu_{\mathrm{Ny}}$. Alias frequencies are frequency values given by the difference between the actual frequency of the signal and integer multiples of the sampling rate. Thus, aliasing allows the detection of true frequencies, even though they occur above $\nu_{\mathrm{Ny}}$ (Murphy, Shibahashi, and Kurtz, 2013).

Equations (9) and (10) reveal that $F_{N}(\nu)$ reaches maxima for alias frequencies $\nu_{j}=j / \Delta t$. Ground-based data are not evenly sampled but they give rise to 1-d and 1-yr alias frequencies whose values of $F_{N}\left(\nu_{j}\right)$ may be similar, preventing from unraveling the "true" frequency from the one introduced by the periodic gaps in the data. This has been a major showstopper for ground-based asteroseismology, particularly in the case of stars with "slow" g modes with periods of approximately $1 \mathrm{~d}$ as in Fig. 3. While these g modes have amplitudes that are easily detectable with ground-based instruments, the 1-d aliasing problems are so severe that one can deduce a few mode frequencies at best, even after yearslong (often multisite) observations; see Zerbi et al. (1999), De Cat and Aerts (2002), Mathias et al. (2004), and Cuypers et al. (2009). This is why
Kepler was so groundbreaking in the field of slow multiperiodic g-mode pulsators. Kepler data also revealed that a good fraction of dwarf pulsators with a convective core (indicated as $\gamma$ Dor, $\delta$ Sct, SPB, and $\beta$ Cep stars in Fig. 1) are actually hybrid pulsators; i.e., they have both short-period (lasting hours) p modes and long-period (lasting days) g modes. Such pulsators have major potential, as their $\mathrm{p}$ and $\mathrm{g}$ modes offer local in situ measurements in different regions in the star: p modes probe the envelope, while $g$ modes probe the radiative near-core regions and allow us to turn the study of deep stellar interiors into observational astronomy.

In practice, applications of frequency analysis to space photometric data are always done after preprocessing and postprocessing of the light curves deduced from the raw data. Satellite repointings (every three months in the case of Kepler, so-called quarters) imply jumps in the time series. Moreover, satellite drift has to be corrected for each quarter. Subsequently, outlier removal and detrending are applied as standard processing to get interpretable light curves (García et al., 2011). Such corrections and processing were applied to get the light curves in the version shown in Fig. 3.

\section{Mode damping and mode lifetimes}

Thus far we have considered a multiperiodic harmonic signal. For stars like those in Fig. 3, this is relatively straightforward because they have modes with extremely long lifetimes. However, a distinction has to be made between two cases: damped modes with lifetimes of the order of or shorter than the duration of the time series $T$ and undamped (or socalled self-excited) modes that never die out and are always active with constant phase throughout the data gathering. The first option occurs for stars such as the Sun with oscillation modes triggered by turbulent motions in their outer convective envelopes. Such "solarlike" oscillations are expected to occur for all stars whose convective envelopes contain sufficient mass to be highly turbulent. These oscillations are of stochastic nature in the sense that they are regularly but randomly excited to more or less the same amplitude, but they damp out relatively quickly, in a time span of the order of days to months, while continuously being reexcited. At each time stamp, the phase within the oscillation cycle is perturbed stochastically relative to the previous measurements.

Stars with radiative envelopes can excite oscillations via a heat mechanism because some of their partial ionization layers manage to transform radiative energy, created in their deep interior by nuclear fusion, into mechanical energy. This can excite modes that resonate inside a mode cavity. This occurs because of local opacity peaks in partial ionization layers of hydrogen, helium, or ironlike species in the outer envelope. Such mode excitation works along the lines of a valve mechanism (also known as thermodynamical Carnot cycle) and may excite radial and nonradial oscillation modes. Because of the key role played by the opacity in getting the modes excited, it is usually called the $\kappa$ mechanism. To a good approximation such resonating modes do not die out as long as radiative energy is delivered to the excitation layer. Hence these modes have "quasi-infinite" lifetimes (i.e., they are infinite to a good approximation compared to the duration of the dataset; hence we drop "quasi" in the text). In such a 
case, it is predictable at what phase in its oscillation cycle the mode will be throughout time.

We return to mode excitation in Sec. III, but in any case we take a data-driven approach to asteroseismology; i.e., we use as many independent eigenmode frequencies as possible, as long as we can extract them from the data, irrespective of how the star managed to excite these oscillations.

\section{Undamped oscillations with quasi-infinite lifetimes}

We first treat the case of heat-driven undamped modes. In this case [see Eq. (3)] we seek to extract the optimal sum of harmonic functions with frequencies $\nu_{1}, \ldots, \nu_{M}$ and amplitudes $A_{1}, \ldots, A_{M}$, where the number of modes $M$ is unknown, keeping in mind the presence of instrumental noise in the data. Under the optimistic assumption of uncorrelated data with white Gaussian noise, a convenient approximation of the Fourier transform is the Lomb-Scargle (LS) periodogram, defined as

$$
\begin{aligned}
P_{\mathrm{LS}}(\nu)= & \frac{1}{2} \frac{\left\{\sum_{i=1}^{N} x\left(t_{i}\right) \cos \left[2 \pi \nu\left(t_{i}-\tau_{0}\right)\right]\right\}^{2}}{\sum_{i=1}^{N} \cos ^{2}\left[2 \pi \nu\left(t_{i}-\tau_{0}\right)\right]} \\
& +\frac{1}{2} \frac{\left\{\sum_{i=1}^{N} x\left(t_{i}\right) \sin \left[2 \pi \nu\left(t_{i}-\tau_{0}\right)\right]\right\}^{2}}{\sum_{i=1}^{N} \sin ^{2}\left[2 \pi \nu\left(t_{i}-\tau_{0}\right)\right]},
\end{aligned}
$$

where $\tau_{0}$ is chosen such that $P_{\mathrm{LS}}(\nu)$ becomes invariant with respect to the starting date of the dataset as follows:

$$
\tan \left(4 \pi \nu \tau_{0}\right)=\frac{\sum_{i=1}^{N} \sin \left(4 \pi \nu t_{i}\right)}{\sum_{i=1}^{N} \cos \left(4 \pi \nu t_{i}\right)}
$$

(Scargle, 1982). Along with the DFT, the LS periodogram is widely used in asteroseismology of stars with heat-driven modes. Both formalisms are suitable to treat gapped nonequidistant time-series data while requiring only a short computation time (Kurtz, 1985). Horne and Baliunas (1986) provided guidance for estimation of the number of independent frequencies as well as a method for detecting the presence of alias frequencies caused by the interaction between the window function and the observed data values. In the limit of $N \rightarrow \infty$, one has $P_{\mathrm{LS}}\left(\nu_{k}\right) \approx A_{k}^{2} N / 4$ for each of the modes $k=1, \ldots, M$. The LS amplitude spectrum $A_{\mathrm{LS}}(\nu) \equiv \sqrt{4 P_{\mathrm{LS}}(\nu) / N}$ thus gives the physically relevant quantities to perform asteroseismology, i.e., the frequencies and amplitudes of the modes. This is the amplitude spectrum shown in red for the seven B-type pulsators in Fig. 3.

Scargle (1982) showed the maxima of $A_{\mathrm{LS}}(\nu)$ to lead to amplitudes $A_{k}$ that are statistically equivalent to those obtained by performing a least-squares optimization to the light curve in the time domain in the limit of large $N$. Even modern datasets may consist of only a limited number of data points. One therefore best performs an optimization to estimate the amplitudes and frequencies once an approximate value of $\nu_{k}$ is known from the DFT or LS periodogram, as a good starting value to perform a least-squares fitting in the time domain. This gives rise to the method of "prewhitening."

We now consider the dominant mode with frequency $\nu_{1}$ deduced from the DFT or LS amplitude spectrum. Minimizing the sum of squares of the residuals

$$
\begin{aligned}
R^{2}\left(\nu_{1}\right) & \equiv \sum_{i=1}^{N}\left[x\left(t_{i}\right)-x^{c}\left(t_{i}\right)\right]^{2} \\
& =\sum_{i=1}^{N}\left[\left(A_{1} \cos \left\{2 \pi\left[\nu_{1}\left(t_{i}-\tau\right)+\psi_{1}\right]\right\}+c\right)\right]^{2}
\end{aligned}
$$

leads to optimized values for $\nu_{1}, A_{1}, \psi_{1}$, and $c$ and provides the residual light curve with an average value of zero

$$
x_{R}\left(t_{i}\right)=x\left(t_{i}\right)-x^{c}\left(t_{i}\right)
$$

A second frequency is then searched for by computing the LS amplitude spectrum for the residual light curve $\left[t_{i}, x_{R}\left(t_{i}\right)\right]$ and optimizing its values $\nu_{2}, A_{2}, \psi_{2}$, etc. This procedure is repeated until the periodogram no longer leads to frequencies that are significant for a specified criterion, such as the ones discussed by Horne and Baliunas (1986), Breger et al. (1993), and Degroote et al. (2009). For a Kepler light curve, this procedure is tedious and time consuming, as it leads to hundreds of significant frequencies. To finalize the list of frequencies due to independent oscillation modes and their uncertainties, great care must be taken to properly account for the occurrence of combination frequencies and harmonics $r \nu_{i}+s \nu_{j}$, with $i, j \in \mathbb{N} ; r$, and $s \in \mathbb{Z}$ due to nonlinearities in the light curve rather than independent mode frequencies, keeping in mind the spectral window and the introduction of spurious frequencies due to limited resolution (Loumos and Deeming, 1978) during the prewhitening process. This was thoroughly discussed by Pápics (2012), Balona (2014), Kurtz et al. (2015), and Bowman (2017). Moreover, one should correct the error estimation of the frequencies and their amplitudes for the correlated nature of the data [as outlined by Degroote et al. (2009) for the highly oversampled CoRoT data]. Although this correction is often omitted, one should apply it to the error estimates of the derived amplitudes, phases, and frequencies. For the case of $N$ data points, one has [cf. Montgomery and O’Donoghue (1999)]

$$
\sigma_{\nu}=\frac{D \sqrt{6} \sigma_{N}}{\pi \sqrt{N} A T}, \quad \sigma_{A}=D \sqrt{\frac{2}{N}} \sigma_{N}, \quad \sigma_{\psi}=\frac{D \sigma_{N}}{\pi \sqrt{2 N A}}
$$

In these three expressions, $\sigma_{N}$ can be approximated by the standard deviation of the final residual light curve. The correction factor due to the correlated nature of the data $D$ depends on the instrument properties and the sampling rate and can be estimated as the square root of the average number of consecutive data points of the same sign in the final residual light curve, as explained by Schwarzenberg-Czerny (1991) and as applied to ground-based photometry by SchwarzenbergCzerny (1998) and to space photometry by Degroote et al. (2009). Values for $D$ are typically between two and ten for the Kepler long-cadence and CoRoT asteroseismology datasets.

\section{Damped oscillations with short lifetimes}

The frequency analysis for stochastically excited damped solarlike oscillations requires a different approach. Because of the random excitation and the damping of the modes, the 
functional form of the light curve changes. The simplest case of one damped oscillation mode with frequency $\nu_{1}$ can be described as

$$
x\left(t_{i}\right)=A_{1} \cos \left[2 \pi\left(\nu_{1} t_{i}+\psi_{1}\right)\right] \exp \left(-\eta_{1} t_{i}\right)+c,
$$

with $\eta_{1}$ the damping rate of the mode, which is the inverse of the mode lifetime. The latter is unknown and hence must be estimated along with the frequency. In the hypothetical case of having continuous observations of such a signal over an infinite amount of time, the power spectrum is given by

$$
P(\nu)=\frac{1}{4} \frac{A_{1}^{2}}{\left[4 \pi^{2}\left(\nu-\nu_{1}\right)^{2}+\left(\eta_{1}\right)^{2}\right]} .
$$

In such a simplified (actually, unrealistic) case, the power spectrum thus takes a Lorentzian profile around the frequency $\nu_{1}$ with the linewidth of the mode given by the full width at half maximum $\Gamma \equiv 2 \eta_{1} / 2 \pi$. Estimation of $\Gamma$ can be accompanied by major uncertainty, unless long and high-quality datasets, such as those assembled by Kepler, are available.

Solarlike oscillation frequencies are usually superimposed onto background power due to low-frequency variability caused by the convective envelope of the star, such as granulation and/or magnetic activity leading to rotational modulation. Stellar granulation occurs in stars with outer convection zones due to the difference between hotter rising gas and cooler downward moving gas. Rotational modulation observed in light or velocity curves is attributed to star spots on the stellar surface, which can have a different temperature, pressure, or chemistry than their surroundings. When the spots have properties that are slowly evolving with time compared to the rotation period, they give rise to rotational modulation at the frequency of the surface rotation and its (sub)harmonics. However, the spots may migrate and/or vary in size over time, leading to low-frequency power excess in the Fourier spectrum.

Both granulation and spots may reach amplitudes that are dominant over those of the oscillations. Any Fourier transform of a light curve undergoing these various aspects of variability will be composed of the superposition of all the harmonic and nonharmonic signals. In such a situation, the method of prewhitening is not meaningful due to the stochastic nature of the variability. Rather, one works with the PD and fits this to extract the oscillation frequencies. In Eq. (17) we have assumed for simplicity that the mode linewidth is independent of frequency. However, it may vary with frequency according to some functional form depending on the mode properties, as discussed for mixed modes in red giants by Mosser et al. (2018).

Different models to describe the oscillation modes and the overall "background" variability were developed and improved as the Kepler data got extended. Kallinger et al. (2014) investigated the PD of a large and homogeneous sample of 1364 stars observed with the Kepler spacecraft, covering almost all evolutionary stages of stars born with a mass between approximately $0.7 M_{\odot}$ and $2.5 M_{\odot}$. All these stars are expected to exhibit stochastically excited oscillations and granulation triggered by their extended convective outer envelope. Hence, Kallinger et al. (2014) searched for one global optimal fitting prescription for the background variability, instead of relying on the background model used for the Sun and adopted in red-giant studies based on early Kepler data releases (Mathur et al., 2011). Kallinger et al. (2014) considered various options for the statistical model formulations of the PD spectra; see their Eq. (2) and Table 1. In line with the findings given by Karoff et al. (2013) for mainsequence stars, the optimal fits to the PD spectra of bright redgiant pulsators require more than one Lorentzian profile to describe the granulation or activity, in addition to a Gaussian power excess caused by oscillations. This is illustrated for one of the red giants in their sample in Fig. 4, where we show the Kepler light curve in the upper left panel, revealing the stochasticity of the variability and the PD in the lower left panel.

Bastien et al. (2013) revealed that the scaling of the granulation amplitude delivers a proper diagnostic for the surface gravity $g$ of the star. The scaling was found to be consistent with that of the pulsation amplitudes. Moreover, the effective temperature has only a marginal additional effect on those amplitudes. This brings us to an important quantity in low-mass star asteroseismology that is further outlined in Sec. IV: the frequency of maximum power $\nu_{\max }$. This quantity was shown to scale to a good approximation as $g T_{\mathrm{eff}}^{-1 / 2}$ by Brown and Gilliland (1994), Kjeldsen and Bedding (1995), and Belkacem et al. (2011). It can be deduced from the Gaussian fit represented by the yellow dotted line in Fig. 4.

Any asteroseismic modeling requires the oscillation frequencies of the damped modes to be extracted from the PD. Various methods and implemented pipelines to do so have been constructed. As said, one does not rely on the method of prewhitening to achieve this. Instead so-called peak-bagging ${ }^{3}$ is done, where the part of interest in the PD is fit with Lorentzian functions for each of the modes as in Eq. (17), either after subtraction of or along with the fit to the granulation background. These methods result in the individual mode frequencies and the mode lifetimes from the linewidths in the PD. Extensive literature covers the methodologies, where the use of a Markov Chain Monte Carlo (MCMC) technique was adopted in this context by Handberg and Campante (2011). Intercomparison of the results obtained by the various methods is taken into account as part of the uncertainties of the mode frequencies, amplitudes, and lifetimes; see Hekker et al. (2011, 2012) and Appourchaux et al. (2012a, 2012b). Particular care of the correlation structure in the frequency analysis of exoplanet host stars was taken using a Bayesian unsupervised approach developed by Davies et al. (2016).

The Kepler observations led to numerical refinements and testing of early analytical expressions for uncertainty estimates of the frequencies of stochastic modes, confirming that they behave as $\sim 1 / \sqrt{T}$ [cf. Eq. (5.57) given by Aerts, Christensen-Dalsgaard, and Kurtz (2010) instead of $\sim 1 / T$

\footnotetext{
${ }^{3}$ This term became standard use in helioseismology and asteroseismology after "peak bagger" Jesper Schou made the analogy between the detection of new solar oscillation frequencies in data obtained from the SoHO satellite and the addition of newly climbed $+14000 \mathrm{ft}$ summits to his personal backpack.
} 

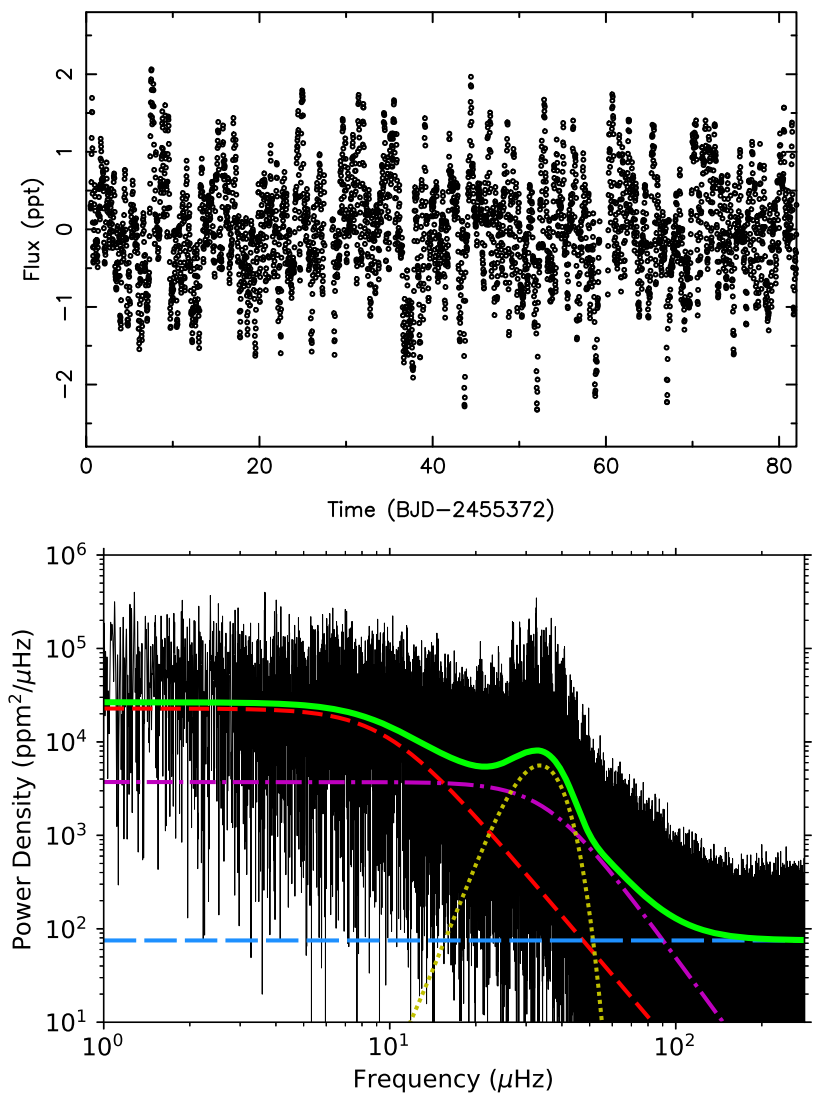
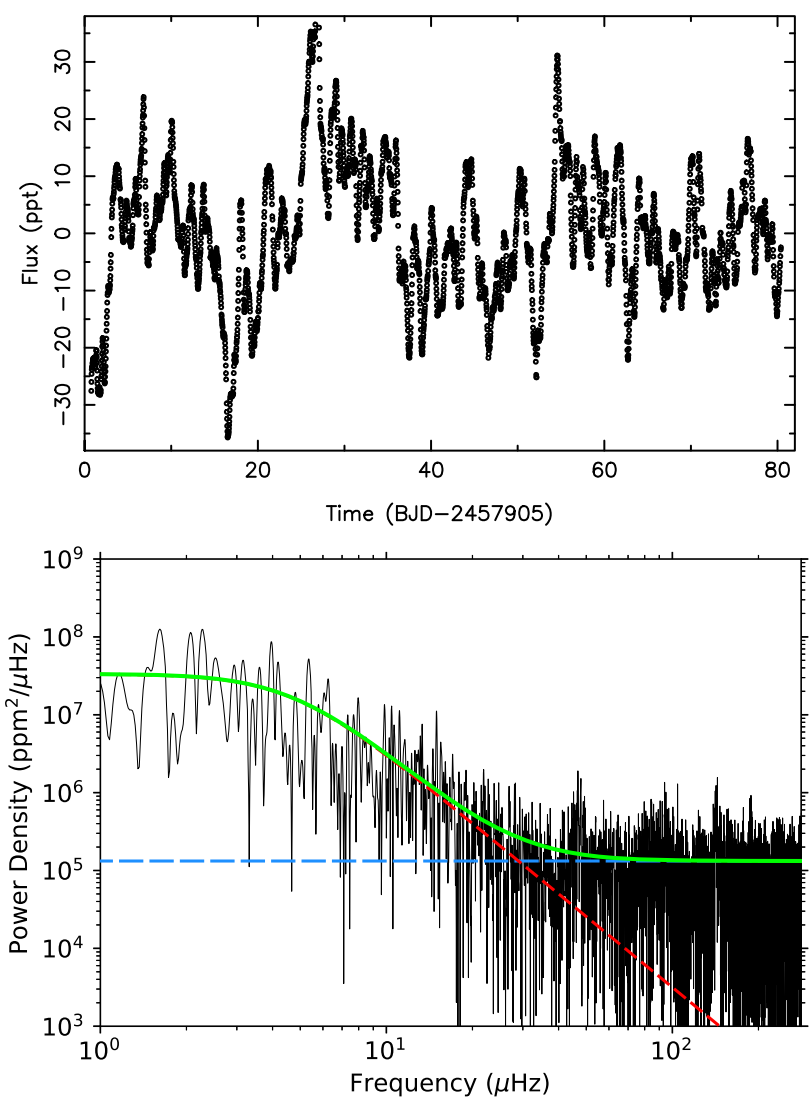

FIG. 4. Part of the Kepler light curve (top left panel) and the power density spectrum (bottom left panel, black) of the red-giant star KIC 007949599. Overplotted in the bottom left panel are the model fits from Table 1 given by Kallinger et al. (2014), based on their Eq. (2), representing two Lorentzian components due to granulation variability at low frequencies (dashed red and dot-dashed purple lines) and a Gaussian component due to stochastic p-mode oscillations (dotted yellow line). K2 light curve (top right panel) and its power density spectrum (bottom right panel, black) of the blue supergiant star $\rho$ Leo. Overplotted in the bottom right is a Lorentzian model fit (dashed red line) using the formalism given by Bowman et al. (2019a) to represent the power excess due to the low-frequency stochastic variability. In both bottom panels, the blue dashed horizontal lines indicate the photon noise level and the solid green lines represent the superposition of the individual model components representing the variability. Adapted from Kallinger et al., 2014, Aerts et al., 2018a, and Bowman et al., 2019b.

as in Eq. (15)]. To get a factor of 2 better frequency precision for damped oscillations one must hence observe 4 times as long, as opposed to only 2 times as long in the case of undamped modes. For elaborate discussions on how the mode linewidths of $\mathrm{p}$ modes were derived from Kepler light curves for dwarfs, subgiants, and red giants, see Chaplin et al. (2009), Appourchaux et al. (2012a), Corsaro, De Ridder, and García (2015), and Vrard et al. (2018). Values translate into mode lifetimes from a fraction of a day for dwarfs to several tens of days for red giants, which is in good agreement with theoretical predictions made by Belkacem et al. (2012). Mosser et al. (2018) succeeded in deriving the mode lifetimes for the challenging case of mixed dipole modes in red giants, with results up to $\sim 100 \mathrm{~d}$. Chaplin et al. (2014) and Yu et al. (2020), respectively, provided summaries of results for about 500 main-sequence stars and 20000 red giants observed with the Kepler spacecraft.

To place the stochastic variability of evolving low-mass stars into a more global context, SLF (Fig. 1) variability was also found to occur in high-mass stars, but for different physical reasons. While all stars develop a convective outer envelope after core-hydrogen burning, young high-mass stars are born with a radiative envelope on top of a convective core. Hence, one does not expect granulation to be present in their envelope. As originally discovered from CoRoT data, young hot O stars reveal SLF in their PD spectra (Blomme et al., 2011). This signature is different from the frequency spectrum for $p$-mode oscillations in $\beta$ Cep stars (cf. Fig. 1), as found for the O9V CoRoT target HD 46202 (Briquet et al., 2011) in the same pointing of the satellite from which the SLF signal was found, so an instrumental cause was excluded. Tkachenko et al. (2014) and Aerts and Rogers (2015) interpreted the signal detected in the space photometry of the close massive binary V380 Cyg and of the O dwarfs as due to convectively driven IGWs. These waves are excited stochastically at the interface between the convective core and the bottom of the radiative envelope in intermediate- and high-mass stars (Rogers et al., 2013). The simulations by Edelmann et al. (2019) and Horst et al. (2020) are representative for young unevolved stars of high mass and deliver frequency spectra of IGWs and eigenmodes as observed in dwarfs with CoRoT and Kepler for stars with masses in the range $[3,25] M_{\odot}$.

Bowman et al. (2019a, 2019b, 2020) performed a systematic study of OBA-type stars from their CoRoT, K2, and TESS data 
to search for SLF, keeping in mind that such a signal is hard to find when it occurs beneath the signal of self-excited modes in the same frequency regime, as for stars in Fig. 3. It was found that SLF variability is ubiquitous in space photometry of most of these stars, irrespective of whether they reside in the Milky Way or in the Large Magellanic Cloud. The K2 light curve and PD spectrum of one such case is shown in the right panels of Fig. 4. It concerns the bright blue supergiant $\rho$ Leo (spectral type B1 Iab) studied by Aerts et al. (2018a) and Pope et al. (2019) at different levels of sophistication. The fit to the PD spectrum of $\rho$ Leo shown in Fig. 4 reveals a characteristic frequency $\nu_{\text {char }}=16.624 \pm 0.007 \mu \mathrm{Hz}$, corresponding to a characteristic timescale of $0.7 \mathrm{~d}$. The K2 light curve of $\rho$ Leo also reveals rotational modulation with a period of $26.8 \mathrm{~d}$ (Aerts et al., 2018a), which corresponds to a frequency of $0.432 \mu \mathrm{Hz}$. The astrophysical interpretation of SLF in intermediate- and high-mass stars may involve a variety of physical causes given the wide range of evolutionary stages covered by the sample.

Armed with the knowledge of how to derive the oscillation frequencies $\omega_{n l m}^{\text {obs }}$ of a pulsating star from high-precision space photometry, we now move on to their exploitation in terms of the star's interior physical properties. This requires that we dive into the theory of nonradial oscillations predicted from equilibrium models of stars. In Secs. II and III, we explain how this can be achieved and under which assumptions. Subsequently we summarize some of the impressive recent results of asteroseismic modeling.

\section{NONRADIAL OSCILLATIONS OF STARS}

The diagnostic power of nonradial oscillations to probe stellar interiors is immense, particularly when compared to observations that probe only the stellar atmosphere. For example, estimates of $T_{\text {eff }}$ of slowly rotating single stars are dependent on atmosphere models and may reach $1 \%$ precision for the best cases. Dynamical masses of binary components are model-independent observables and may reach 1\% accuracy; see Serenelli et al. (2021) for a summary. Oscillation frequencies can be measured directly from data, without any model dependence, at the level of $0.001 \%$ for $\mathrm{p}$ modes of low-mass stars and $0.1 \%$ for $\mathrm{g}$ modes of intermediate-mass stars; see Aerts, Mathis, and Rogers (2019), Table 1. These precisions of mode frequencies lie at the heart of the revolution brought by space asteroseismology.

The interpretation of detected oscillation modes requires a good understanding of the theory of nonradial oscillations and how the modes depend on the properties of stellar interiors. This dependence is currently studied from numerical computations of stellar equilibrium models and their predicted oscillations, for different sets of input physics and free parameters. However, various forms of analytical expressions for the mode properties are highly insightful for the understanding of the mode behavior. In fact, asymptotic approximations of the mode frequencies offer an important basis to interpret the observations, even in current times of large computational power. This was stressed by Cunha et al. (2015) and illustrated by Van Reeth, Tkachenko, and Aerts (2016), Ouazzani et al. (2017), Christophe et al. (2018), and Cunha, Avelino et al. (2019).
Extensive textbooks on the theory of nonradial oscillations of stars were produced by Unno et al. (1989) and Smeyers and Van Hoolst (2010), to which we refer for the historical developments and for mathematical derivations. Here we limit ourselves to the bare minimum required to understand applications of asteroseismology. Aerts, Christensen-Dalsgaard, and Kurtz (2010) provided detailed descriptions of the general methodology and applications covering all masses and types of nonradial oscillations, while Basu and Chaplin (2017) covered applications based on space photometry but limited to stochastically excited solarlike oscillations. Tong and García (2015) covered synergies between planetary and stellar seismology. As outlined in the Introduction, the asteroseismology revolution of the past decade is so immense that we focus the rest of this review on applications based on the recent space photometry, even though this does injustice to numerous studies and efforts prior to 2010 .

\section{A. Stars and their hydrodynamics}

The equations describing the oscillations of stars are perturbed versions of the equations of hydrodynamics applied to a gaseous self-gravitating sphere. We introduce the basics of stellar hydrodynamics before moving on to stellar oscillations. We omit the derivation of these basic equations here, as this is the topic of various books on fluid dynamics. A seminal introduction to hydrodynamics with specific attention to stellar oscillations was given by Ledoux and Walraven (1958). Here we limit ourselves to the ingredients needed to move on to asteroseismic modeling while omitting unnecessary details.

\section{The stellar structure equations}

The equations to be solved to compute stellar models throughout the evolution of stars are the general equations of physics, expressing conservation of mass, momentum, and energy. In stellar interiors, the circumstances are such that viscosity can be ignored and the conservation laws can be limited to gaseous objects. The derivation of the equations expressing the conservation laws for stellar structure and evolution was covered by Cox and Giuli (1968), Hansen, Kawaler, and Trimble (2004), Maeder (2009), and Kippenhahn, Weigert, and Weiss (2012).

Conservation of mass leads to the equation

$$
\frac{\partial \rho}{\partial t}+\nabla(\rho \boldsymbol{v})=0
$$

where $\rho(\boldsymbol{r}, t)$ is the local density at position vector $\boldsymbol{r}$ and $\boldsymbol{v}(\boldsymbol{r}, t)$ is the local velocity vector, both at time $t$. The equations of motion, expressing conservation of momentum, can be written as

$$
\rho \frac{\partial \boldsymbol{v}}{\partial t}+\rho \boldsymbol{v} \cdot \nabla \boldsymbol{v}=-\nabla p-\rho \nabla \Phi+\rho \boldsymbol{f}
$$

where $f$ is body force per unit mass and $\Phi$ is the gravitational potential satisfying the Poisson equation

$$
\nabla^{2} \Phi=4 \pi G \rho
$$


and where it is assumed that internal friction in the gas can be ignored (i.e., zero viscosity). In general, $\boldsymbol{f}$ stands for the electromagnetic and possibly external forces such as tidal forces in multiple systems. The energy equation is derived from the thermodynamical properties and the energetics of the gas and can be formulated as

$$
\rho T \frac{\partial S}{\partial t}+\rho T \boldsymbol{v} \cdot \nabla S=\rho \varepsilon-\nabla \boldsymbol{F},
$$

with $S$ the entropy per unit mass, $\varepsilon$ the energy generation rate per unit mass taking into account the energy loss from neutrinos, and $\boldsymbol{F}$ the energy flux.

Further, an equation for the overall energy transport throughout the star needs to be added. This is fairly straightforward in radiative zones of the star, because the mean free path of a photon is ultrashort compared to the length scales over which the stellar structure changes $(\sim 2 \mathrm{~cm}$ in the solar interior, for instance). In such a case the radiative energy transport is well described by a diffusion approximation. For stellar interiors, this is given by

$$
\boldsymbol{F}=-\frac{4 \pi}{3 \kappa \rho} \nabla B=-\frac{4 a c T^{3}}{3 \kappa \rho} \nabla T
$$

where $B=(a c / 4 \pi) T^{4}$ results from integrating Planck's radiation function, $\kappa$ is the flux-weighted opacity, $c$ is the speed of light, and $a$ is the radiation density constant.

In convection zones of the stellar interior, the turbulent gas motions transport the energy in an efficient yet complex manner. In the absence of a proper theory for the dynamical effect of convection for stellar interiors, the turbulent pressure is usually ignored and the treatment of convective energy transport in stellar evolution codes is time independent. This approach is a crude approximation: it is based on pragmatism rather than sophistication. Although various versions exist for the description of convective instabilities, the most popular treatment of time-independent convection is the so-called mixing-length theory (mlt); see Houdek and Dupret (2015) for a historical overview. It is characterized by the free parameter $\alpha_{\text {mlt }}$ (expressed in units of the local pressure scale height $H_{p}$ ), which stands for the mean free path over which the convective eddies travel before dissolving in their environment. Asteroseismology allows one to infer the extent of convective regions via estimation of the free parameters of the convection formulation used for the modeling. This was done for mlt by Joyce and Chaboyer (2018) and Viani et al. (2018).

Whenever the diffusion of photons is insufficiently efficient as an energy transport mechanism, convection not only takes over the energy transport but also changes the temperature gradient relative to the radiative one in Eq. (22). From a computational point of view, the calculation of the energy transport must hence be split up for the radiative and convective zones inside the stellar model. This is done by testing whether a zone with temperature gradient

$$
\nabla \equiv \frac{\mathrm{d} \ln T}{\mathrm{~d} \ln p}
$$

is stable or unstable against convection. The general condition to test for convective stability is the so-called Ledoux criterion

$$
\nabla_{\mathrm{rad}}<\nabla_{\mathrm{ad}}+\frac{\varphi}{\delta} \nabla_{\mu}
$$

where we have introduced

$$
\begin{aligned}
\nabla_{\mathrm{rad}} & =\frac{3}{16 \pi a c G} \frac{\kappa p}{T^{4}} \frac{L(r)}{m(r)}, \quad \nabla_{\mathrm{ad}}=\left(\frac{\partial \ln T}{\partial \ln p}\right)_{S}, \\
\nabla_{\mu} & =\frac{\mathrm{d} \ln \mu}{\mathrm{d} \ln p}, \quad \delta=-\left(\frac{\partial \ln \rho}{\partial \ln T}\right)_{p, \mu}, \quad \varphi=\left(\frac{\partial \ln \rho}{\partial \ln \mu}\right)_{p, T},
\end{aligned}
$$

with $\mu$ the mean molecular weight of the ionized gas. For zones with a homogeneous chemical composition, the Ledoux criterion reduces to the Schwarzschild criterion

$$
\nabla_{\mathrm{rad}}<\nabla_{\mathrm{ad}}
$$

Stars born with a mass above $\sim 1.7 M_{\odot}$ have a receding convective core as they evolve throughout the core-hydrogen-burning phase because the opacity $\kappa$ decreases as the hydrogen depletes, reducing $\nabla_{\text {rad }}$. The resulting composition gradient gives rise to $\nabla_{\mu} \neq 0$ and increases stability in that zone. On the other hand, the change of $\nabla_{\text {rad }}$ for stars born with a mass below $\sim 1.3 M_{\odot}$ is dominated by the factor $L(r) / m(r)$, which increases faster than $\kappa$ decreases. The interplay in importance between $\kappa$ and $L(r) / m(r)$ in the expression of $\nabla_{\text {rad }}$, and along with it the growth or shrinkage of the convective core, depends on the physical circumstances for masses between $1.3 M_{\odot}$ and $1.7 M_{\odot}$ (Mombarg et al., 2019).

In a zone that is stable against convection, a fluid element that gets displaced by moving up will be pulled back down until it is again situated at its equilibrium position, thanks to the action of the buoyancy force of Archimedes. This oscillatory motion of the fluid elements depends on the local density, pressure, and chemical composition of the gas and happens with the so-called Brunt-Väisälä frequency, or buoyancy frequency for short, which can be approximated as

$$
N^{2} \simeq \frac{g}{H_{p}}\left[\delta\left(\nabla_{\mathrm{ad}}-\nabla\right)+\varphi \nabla_{\mu}\right]
$$

with $g$ the local gravity. The $\mu$ gradient affects the local behavior of $N(r)$ in the radiatively stratified layers of the star. As discussed later, this will affect stellar oscillations, notably internal gravity waves. In the case of instability, i.e., $N^{2}<0$, the speed of the fluid element increases exponentially with time until it breaks up, causing complete and instantaneous mixing of the chemical species.

Even though Eq. (24) allows us to derive the zones where convection takes place inside the star, complications occur in the transition layers between convective and radiative zones, hereafter termed convective boundary layers. The fluid elements inside a convection zone experience a turbulent motion with velocity $\boldsymbol{v}_{\text {conv }}$. When they reach the convective boundary layer, their inertia will prevent them from stopping abruptly; i.e., they will "overshoot" from the convection zone into the radiative layer over an unknown distance, which we denote as 
$\alpha_{\mathrm{ov}}$ (in analogy to $\alpha_{\mathrm{mlt}}$, it is expressed in the unit $H_{p}$ ). The way in which the fluid elements overshoot the convective boundary depends on the location of the convection zone inside the star and the physical circumstances at that position. For extensive discussions, see Zahn (1991), Viallet et al. (2015), Cristini et al. (2016), Constantino, Campbell, and Lattanzio (2017), and Arnett et al. (2019). Three-dimensional simulation studies have indicated at least three physical processes that may come into play: penetration by plumes leading to superadiabatic mixing over a distance $d_{\text {pen }}$ (Zahn, 1991), subadiabatic thermal diffusion over a distance described by means of an exponentially decaying mixing profile with parameter $f_{\text {ov }}$ (Freytag, Ludwig, and Steffen, 1996; Herwig, 2000), or turbulent entrainment that occurs over a dissipation length scale expressed as a distance $l_{\mathrm{d}}$ (Meakin and Arnett, 2007; Viallet et al., 2013). These imply a different and uncalibrated level and functional form of convective boundary mixing (CBM) and have a different temperature gradient in the transition layer. We use the notation of the free parameter $\alpha_{\mathrm{ov}}$ to express the unknown length scale over which the fluid elements move from inside a convective region into the radiative adjacent zone, representing any of $d_{\text {pen }}, f_{\text {ov }}, l_{\mathrm{d}}$, or other formulations; cf. Augustson and Mathis (2019).

The rate of change of species of type $i$ with relative mass fraction $X_{i}$ is caused by various processes, some of which are diffusive but others that are not. When the rate of change happens much faster than the nuclear timescale, it is customary to approach $\partial X_{i} / \partial t$ by a diffusion equation for computational convenience. In the simplest case of changes due to convective motions, along with nuclear fusion in a spherically symmetric star, we can write

$$
\frac{\partial X_{i}}{\partial t}=\mathcal{R}_{i}+\frac{1}{\rho r^{2}} \frac{\partial}{\partial r}\left[\left(D_{\text {conv }}+D_{\text {ov }}\right) \rho r^{2} \frac{\partial X_{i}}{\partial r}\right]
$$

where the rate of change of $X_{i}$ due to nuclear reactions is denoted symbolically as $\mathcal{R}_{i}$. The diffusion coefficient associated with the convective mixing described by mlt is given by

$$
D_{\text {conv }}=\frac{1}{3} \alpha_{\text {mlt }} H_{p} v_{\text {conv }} \text {. }
$$

The unknown profile of CBM due to the overshooting of the fluid elements beyond the convective boundary is denoted here as $D_{\text {ov }}$. Each of the profiles $D_{\text {conv }}(r)$ and $D_{\text {ov }}(r)$ (expressed in the physical units $\mathrm{cm}^{2} \mathrm{~s}^{-1}$ ) is in general an unknown function of $r$ and involves at least one free parameter $\left(\alpha_{\text {mlt }}\right.$ and/or $\left.\alpha_{\text {ov }}\right)$.

For stars with a convective core, the lack of calibration of the physics in the convective boundary layers implies a serious limitation. Indeed, the CBM influences the amount of matter that can be brought into the central regions where nuclear fusion takes place. The higher the CBM, the more fresh fuel reaches the nuclear reactor and hence the longer the nuclear fusion can go on. This has a major impact on the star's core mass and its age. For this reason, calibration of the amount of matter in the convective core of a star $M_{\mathrm{cc}}$, via an observational estimation of the profile $D_{\mathrm{ov}}(r, t)$ and its feedback throughout the evolution of the star, is a crucial piece of information to predict a star's life and age. It was shown that space asteroseismology has the capacity to deliver such an estimation across a large mass range by Deheuvels et al. (2016) and Pedersen et al. (2018), including assessment of the temperature gradient in the near-core boundary layer (Michielsen et al., 2019). This potential had already been pointed out by Dziembowski and Pamyatnykh (1991) but remained without practical application until recently. Concrete applications to derive $D_{\mathrm{ov}}(r)$ based on space asteroseismology are discussed in Sec. IV.

\section{Simplification to 1D stellar models}

Because of the immense range in timescales and spatial scales occurring in the interiors of stars, stellar models must necessarily remain a simplified version of reality. Indeed, the computation of 3D models across stellar evolution is not yet feasible. One thus needs to adopt simplifications in the computation of stellar structure models. With asteroseismic applications in mind, we make two important approximations: we assume that any equilibrium model, which will be perturbed to compute a star's oscillations, is spherically symmetric and does not have a dynamical atmosphere. The first simplification implies that we can rely on 1D models in hydrostatic equilibrium computed for stars that do not rotate close to their so-called critical rotation rate. The second simplification allows us to use a static atmosphere model to connect to the stellar interior as outer boundary at each time step in a star's evolution.

In practice, stellar evolution codes rely on mass-loss or accretion rates described by parametrized laws, such that an amount $\dot{M} \Delta t$ is peeled off or added to the stellar model after a duration $\Delta t$ of stellar life has passed. For each particular instance in time, the stellar model is considered to have a static atmosphere on top of its interior structure. In this way, the models are built while taking mass loss or accreted mass into account and ignoring the dynamical properties due to a stellar wind or an accretion disk. This simplifies the boundary conditions adopted to close the set of equations to be solved. This basic assumption is justified for the majority of applications in asteroseismology, because nonradial oscillations are usually undetectable for stars that have a strong dynamical wind or high levels of accretion.

Ignoring fast rotation needs more justification than neglecting the dynamics of the wind, because rotation is common in stars throughout their lives. Rotation acts upon stellar structure in at least three ways: it deforms the star from spherical symmetry, it leads to higher polar than equatorial flux due to gravity darkening, and it induces various instabilities and mixing in the stellar interior. The level of confidence in how to treat these effects is different for the three aspects. Gravity darkening was first discussed by von Zeipel (1924). It stands for a reduction in the flux and hence in the effective temperature of the star resulting from the reduced gravity in the equatorial regions relative to the polar ones. The von Zeipel effect is expressed as

$$
T_{\text {eff }}=T_{\text {eff }, \mathrm{p}}\left(\frac{g_{\text {eff }}}{g_{\text {eff }, \mathrm{p}}}\right)^{\beta},
$$


where $T_{\text {eff,p }}$ and $g_{\text {eff,p }}$ are the effective temperature and effective gravity at the pole of the star. For a radiative envelope as considered by von Zeipel, $\beta \simeq 0.25$. In the presence of a convective envelope, $\beta$ is usually assumed to be $\beta<0.1$. This limited knowledge of the exponent $\beta$, and along with it a nonsymmetrical stellar wind, implies a nontrivial treatment in stellar evolution computations in the presence of rotation.

By definition, the critical (or breakup) velocity is reached when the outwardly directed centrifugal acceleration is equal to the inward effective gravitational acceleration at any one point of the stellar surface. Here we work with the critical rotation frequency since we are making a comparison to the frequencies of oscillations. Usually the Roche approximation is adopted, which assumes that the mass concentration inside the star is not distorted by the rotation. In this case, the polar $\left(R_{\mathrm{p}}\right)$ and equatorial $\left(R_{\mathrm{e}}\right)$ radii of the star differ by less than a factor of $3 / 2$, where $R_{\mathrm{e}, \text { crit }} / R_{\mathrm{p}, \text { crit }}=3 / 2$. This leads to the critical rotation frequency given by $\Omega_{\text {crit }}=\sqrt{G M_{\star} / R_{\mathrm{e}, \text { crit }}^{3}}=$ $\sqrt{8 G M_{\star} / 27 R_{\mathrm{p}, \text { crit }}^{3}}$, with $M_{\star}$ the mass of the star and $R_{\mathrm{e}, \text { crit }}, R_{\mathrm{p}, \text { crit }}$ its critical equatorial and polar radii. This is the solution for the critical rotation frequency when the Eddington parameter $\Gamma=\kappa L / 4 \pi c G M_{\star}<0.639$ (Maeder, 1999). The other solution is not considered here, as almost all applications in asteroseismology thus far occur for stars without a strong radiation-driven wind.

The prediction $R_{\mathrm{e}, \text { crit }} / R_{\mathrm{p}, \text { crit }}<3 / 2$, along with von Zeipel's formula (29), can be evaluated directly from interferometric measurements of stellar surfaces. Such observations indeed show that fast rotators are oblate (Domiciano de Souza et al., 2018) and that their surface properties and winds are not spherically symmetric, as revealed by Kervella and Domiciano de Souza (2006) and Domiciano de Souza et al. (2014). However, fast rotating stars do not necessarily comply with the Roche approximation. Interferometry of the Be star Archernar (Domiciano de Souza et al., 2003) led to $R_{\mathrm{e}} / R_{\mathrm{p}} \simeq 1.56$. Moreover, von Zeipel's law is not adhered to by the stars having gravity darkening measurements from interferometry (Domiciano de Souza et al., 2018). This led Gagnier et al. (2019) to perform a new evaluation of the critical rotation of a star from 2D static models, with the conclusion that $\beta$ decreases from 0.25 to 0.13 for rotation rates evolving from slow to critical.

In conclusion, the computation of 2D equilibrium models in the presence of rotation comes with major uncertainty, even in its simplest aspects of the local surface and its flux. For this reason, computations of stellar oscillations from $2 \mathrm{D}$ equilibrium models are often restricted to static polytropic models (Lignières, Rieutord, and Reese, 2006; Reese, Lignières, and Rieutord, 2006). Although progress is steadily achieved (Ouazzani, Dupret, and Reese, 2012; Reese et al., 2013), fitting of measured frequencies to perform $2 \mathrm{D}$ asteroseismic modeling is not within reach yet. We thus do not treat $2 \mathrm{D}$ equilibrium models as input for oscillation-mode computations. Rather, we focus in this review on tuning the stellar interior quantitatively by taking into account the Coriolis acceleration at the level of the mode computations while relying on $1 \mathrm{D}$ equilibrium models. Lignières, Rieutord, and
Reese (2006), Ballot et al. (2010), and Ouazzani et al. (2017) provided comparisons between oscillation frequency predictions of rapid rotators from $1 \mathrm{D}$ versus $2 \mathrm{D}$ equilibrium models using higher-order perturbative expressions for the effect of rotation. These studies revealed that a 1D treatment for $\mathrm{p}$ modes leads to appropriate oscillation predictions for stars rotating up to $\sim 15 \%$ of the critical rotation frequency. For high-order g modes, the 1D treatment is justified up to 70\% of the critical rotation frequency (Henneco et al., 2021). Within these regimes, it is justified to work with 1D equilibrium models as input for the computation of oscillation modes, where no rotation or only the spherically symmetric component of the centrifugal force is included in Eq. (19). In the latter case, the simplified equation of hydrostatic equilibrium reads

$$
\frac{\partial p}{\partial r}=-\frac{G m \rho}{r^{2}}+\frac{2}{3} \rho r \Omega^{2} .
$$

\section{Standard 1D stellar models in hydrostatic equilibrium}

Thus far we have focused on the internal structure of the star, but we have hardly considered its chemical evolution. The chemical composition inside the star at time $t$ is described by relative mass fractions of species $i, X_{i}=X_{i}(r, t)$, where $r \in\left[0, R_{\star}(t)\right]$, with $R_{\star}(t)$ the radius of the spherically symmetric star at time $t$ in its evolution. These profiles are an important aspect of stellar models, because they determine the opacity, thermodynamical characteristics, and energy production $\varepsilon$ due to nuclear reactions as in Eq. (21). These reactions, in turn, change the chemical composition and rule the life of the star.

To solve the stellar structure equations (18)-(21) along with the energy transport equation(s) and the changes in the chemical profiles $X_{i}=X_{i}(r, t)$, the microscopic properties of the stellar matter need to be known as a function of $p(r, t)$, $T(r, t)$, etc. This requires adopting an equation of state, various thermodynamical properties, opacity tables to compute the Rosseland mean opacity, a network of nuclear reaction rates, etc. This is jointly referred to as "input physics" when one computes stellar models. Further, proper boundary conditions at the center and surface of the star and initial conditions characterizing the star's properties at birth $(\tau \equiv 0)$ when it has arrived at the so-called zero-age main sequence (ZAMS) have to be chosen [this is not discussed here; see Kippenhahn, Weigert, and Weiss (2012)]. The solution of the equations for chosen boundary conditions delivers what is called a stellar equilibrium model at age $\tau$ described by $m(r, \tau), p(r, \tau), L(r, \tau), T(r, \tau), X_{i}(r, \tau)$, and by all other relevant functions that can be derived from these solutions, with $m(r, \tau)$ the mass enclosed by the shell positioned at $r \in$ $\left[0, R_{\star}(\tau)\right]$ inside the star.

The ZAMS $\tau \equiv 0$ is defined as the point in time when hydrogen fusion occurs in full equilibrium in the center of the star. At the ZAMS, the star has a specific yet unknown chemical mixture of species $X_{i}$ in its interior. This mixture is the result from the initial chemistry it received from its birth cloud when it started as a fully convective protostar on the socalled Hayashi track [see Kippenhahn, Weigert, and Weiss (2012) for a definition], and from changes in this mixture due 
to nuclear reactions and due to mixing taking place during the contraction phase from the Hayashi track toward the ZAMS. Often, the computation of stellar models for low-mass stars adopts the solar mixture using the Sun's current or initial surface abundances (Asplund et al., 2009). On the other hand, the surface abundances of B-type stars in the solar neighborhood (Przybilla et al., 2013) constitute a logical choice for the initial chemical mixture when computing high-mass stellar models. With a specified chemical mixture, the initial composition is an input for the 1D evolutionary computations. We denote this initial composition as $X_{\text {ini }}, Y_{\text {ini }}, Z_{\text {ini }}$, which stand for the initial hydrogen, helium, and metal mass fractions, complying with $X_{\text {ini }}+Y_{\text {ini }}+Z_{\text {ini }}=1$. For most of the phases of stellar evolution, the stars do not change on a dynamical timescale, as it is much shorter than the contraction timescale and the nuclear timescale. Whenever this is the case, the lefthand side of Eq. (19) is zero and the resulting stellar model is in hydrostatic equilibrium.

In the simplest case of a nonrotating nonmagnetic single star without a stellar wind, there is no additional body force $f$ in Eq. (19) such that the pressure and gravity forces are the actors that compensate for each other. Such simplifications lead to equilibrium models that resemble reality well for many of the stars and during large fractions of their life. Stellar models computed with those simplifications for the interior and with a static atmosphere as outer boundary are called standard stellar models. Evolutionary tracks representing such standard models are included as full lines in Fig. 1. Extensive comparisons of stellar evolution models computed with independently developed codes have been done for low-mass stars in the context of CoRoT (Monteiro, 2009) and show impressive agreement when the same input physics is considered. This is in sharp contrast to the major differences occurring for stellar evolution computations based on similar input physics for high-mass stars, even for nonrotating models (Martins and Palacios, 2013).

\section{Nonstandard 1D models with microscopic atomic diffusion}

Composition changes do not occur only in regions where nuclear reactions take place. In addition to full and instantaneous mixing in convective regions and full or partial mixing in convective boundary layers, the chemical profiles in radiative regions may also change due to microscopic and macroscopic transport processes (Pinsonneault, 1997; Salaris and Cassisi, 2017). Which of those is dominant depends on the timescales upon which they act. Macroscopic mixing may be induced by turbulence, magnetic fields, waves, rotation, etc. In this section, we focus on the microscopic scale and consider element transport caused by microscopic atomic diffusion. The accompanying local chemical composition changes induced by it are caused by gradients operating in the radiative layers of the star. These gradients may introduce lower or higher concentrations of particular chemical species, in particular, layers of the radiative envelope. Section II.A.5treats macroscopic element transport.

A key aspect of assessing the importance of microscopic diffusion is that the timescales upon which it acts are significantly different for the atmosphere than for the interior of the star (Michaud, Alecian, and Richer, 2015). Diffusion timescales are typically less than a century for the stellar atmosphere, while they are millions to billions of years for the interior regions. Given that we focus on asteroseismic applications and on the tuning of stellar interiors, we do not consider modeling surface abundances affected by atomic diffusion as observed in some intermediate-mass stars (socalled Ap and Bp stars). Rather, we limit ourselves to those aspects of atomic diffusion that act on long timescales in radiative parts of the stellar interior, keeping in mind the importance of atomic diffusion for the solar case, as demonstrated from helioseismology by Christensen-Dalsgaard, Proffitt, and Thompson (1993).

Following numerous studies (Thoul, Bahcall, and Loeb, 1994; Chayer, Fontaine, and Wesemael, 1995; Richer, Michaud, and Turcotte, 2000; Richard, Michaud, and Richer, 2002; Richard et al., 2002; VandenBerg et al., 2002; Michaud et al., 2004; Hu et al., 2011; Théado et al., 2012; Deal, Richard, and Vauclair, 2016), four different aspects of microscopic atomic diffusion are considered in stellar models. These occur due to pressure, temperature, and concentration gradients, on the one hand, and radiative forces, on the other hand. While pressure and temperature gradients augment the concentration of more massive species toward the center of the star, concentration gradients have the opposite effect. On the other hand, radiative forces levitate species with an efficiency that depends on the details of the atomic structure of the involved isotopes. The calculation of the appropriate radiative accelerations is therefore challenging in terms of computation times. The accelerations can be computed from atomic data by treating the appropriate multicomponent gas (Burgers, 1969). This requires evaluations of the frequency-dependent absorption coefficients derived from a screened Coulomb potential (Paquette et al., 1986), taking into account partial ionization, and this for all the layers inside the star; cf. Thoul, Bahcall, and Loeb (1994). Once the overall local velocities $w_{i}$ for each of the species $i$ involved in the atomic diffusion are computed, they can be inserted into the following equation governing the time evolution of the mass fraction $X_{i}$ :

$$
\begin{aligned}
\frac{\partial X_{i}}{\partial t}= & \mathcal{R}_{i}-\frac{1}{\rho r^{2}} \frac{\partial}{\partial r}\left(\rho r^{2} X_{i} w_{i}\right) \\
& +\frac{1}{\rho r^{2}} \frac{\partial}{\partial r}\left[\left(D_{\text {conv }}+D_{\text {ov }}\right) \rho r^{2} \frac{\partial X_{i}}{\partial r}\right]
\end{aligned}
$$

where the second term on the right-hand side is the result of the microscopic atomic diffusion acting upon species $X_{i}$ and the third term is the result of macroscopic transport of the chemical species due to convection and overshooting.

If atomic diffusion can be treated without the radiative effects, which is a good approximation for cool stars with extended convective envelopes such as the Sun, its impact on the computation time required for evolutionary model calculations is modest. As a consequence, the use of such simplified microscopic diffusion computations without levitation in evolutionary models is widespread (Chaboyer, Demarque, and Pinsonneault, 1995; Pinsonneault, 1997). With levitation included, the computation of $w_{i}$ and the solution of the set of equations (31) at each step of the evolution is a major 


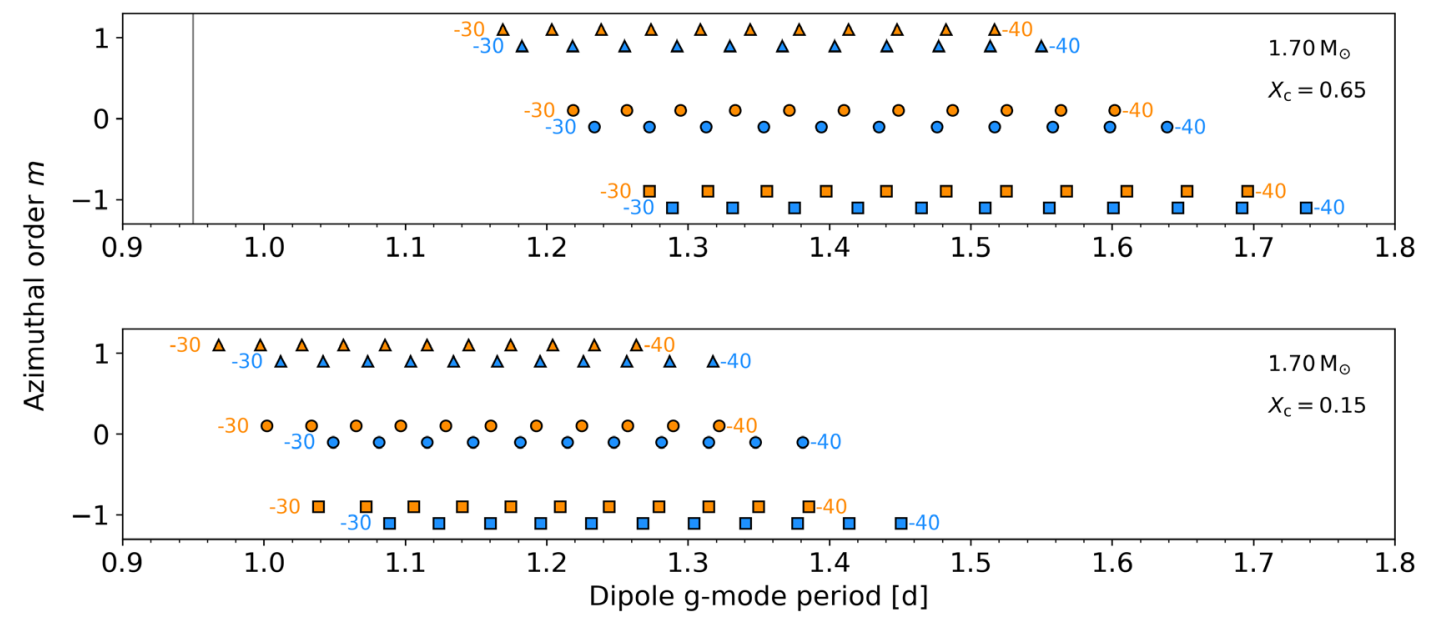

FIG. 5. Shifts in the periods of dipole $(l=1)$ triplet g modes of two equilibrium models [blue (orange), without (with) atomic diffusion, including radiative levitation] with the same input physics for parameters $M=1.7 M_{\odot}, X_{\text {ini }}=0.7154, Z_{\text {ini }}=0.022$, and at two different evolutionary stages expressed in terms of the central hydrogen fraction $X_{c}$. The g modes were computed assuming rigid rotation with a period of $14.4 \mathrm{~d}$. The radial orders $n$ are labeled. The vertical line indicated on the left side in the upper panel denotes the measurement uncertainty for such mode periods from a 4-yr nominal Kepler light curve. Adapted from Mombarg et al., 2020.

challenge. Nevertheless, such computations have been done with the specific aim of asteroseismic applications, adopting various levels of complexity. Studies of stellar interiors of Aand F-type stars were given by Turcotte, Richer, and Michaud (1998), Deal et al. (2018), and Verma and Silva Aguirre (2019), subdwarf B stars were studied by Hu et al. (2011) and Bloemen et al. (2014), and white dwarfs were studied by Romero et al. (2017) and De Gerónimo et al. (2019), where the last two papers did not include radiative levitation. Figure 5 shows the influence of atomic diffusion on g-mode frequencies of intermediate-mass stars whose rotation period is about 10 times longer than its dipole-mode periods. The frequency shifts induced by atomic diffusion are much larger than the measurement uncertainties, highlighting the fact that asteroseismology has the capacity to evaluate the need (or not) of radiative levitation in models of such stars, as illustrated by Mombarg et al. (2020).

It is instructive, particularly for the later nuclear burning stages, to compare the asteroseismic results based on evolutionary models with those obtained from static structure models that are more sophisticated in some aspects of the structure yet less prone to unknown aspects of the physics in the models that accumulate throughout the evolution. This approach was followed by Charpinet et al. (2011) and Van Grootel et al. (2013), as well as by Giammichele et al. (2018) and Charpinet et al. (2019) for subdwarfs and white dwarfs, respectively. Differences in the stellar structure profiles from such static models $\left[m(r), p(r), T(r), L(r)\right.$, and $\left.X_{i}(r)\right]$ compared to those obtained from evolutionary models can then be used to improve the input physics adopted for full evolutionary computations via an iterative loop between asteroseismology and the equilibrium models, as given by Timmes et al. (2018) and De Gerónimo et al. (2019).

Atomic diffusion impacts the concentration of the species in the stellar interior on timescales that are relevant for stellar evolution. Its effect is hard to unravel from a star's luminosity and effective temperature, which are the two quantities that define the evolutionary tracks in a HRD. Models with and without atomic diffusion (either with or without levitation) usually differ far less than typical observational errors of $L$ or $\log g$ plotted versus $T_{\text {eff }}$, as shown by Dotter et al. (2017) and Deal et al. (2018). As a confrontation between data and theory in the HRD is commonly the only assessment to evaluate stellar evolutionary theory, and given the computational requirements, microscopic atomic diffusion is often ignored in stellar and galactic astrophysics. Its inclusion is, however, critical when evaluating surface abundances for archaelogical chemical tagging (Dotter et al., 2017) and to interpret asteroseismic data, as was done by Verma et al. (2017), Deal et al. (2018, 2020), and Mombarg et al. (2020).

\section{Nonstandard 1D models with rotation and waves}

Rotation has a major effect on stellar evolution (Maeder, 2009). Yet in this era of space asteroseismology, it has become clear that its treatment in stellar interiors is up for improvement. Computations based on local conservation of angular momentum and rotational mixing, both of which have been used extensively in stellar evolution models the past few decades, lead to predictions that are incompatible with asteroseismology, as discussed later. Even 1D models of slowly rotating stars face challenges. Improving them is a major aim of asteroseismology, and of stellar astrophysics in general. We have now reached the stage where asteroseismic inferences based on high-quality space photometry can be used to derive $\Omega(r)$ for stars and to provide a calibration of the poorly known physical ingredients of rotating stellar models, which is in line with Gough's quote in the Introduction: that is, asteroseismology in action.

To compute equilibrium models including rotation, we need to know $\Omega(r)$ and how it changes throughout stellar evolution. Asteroseismology is a major and thus far unique game changer on this front. While we discuss methods to deduce $\Omega(r)$ in Sec. IV.D, we provide the current status of the rotation frequencies in the region just outside the convective core 

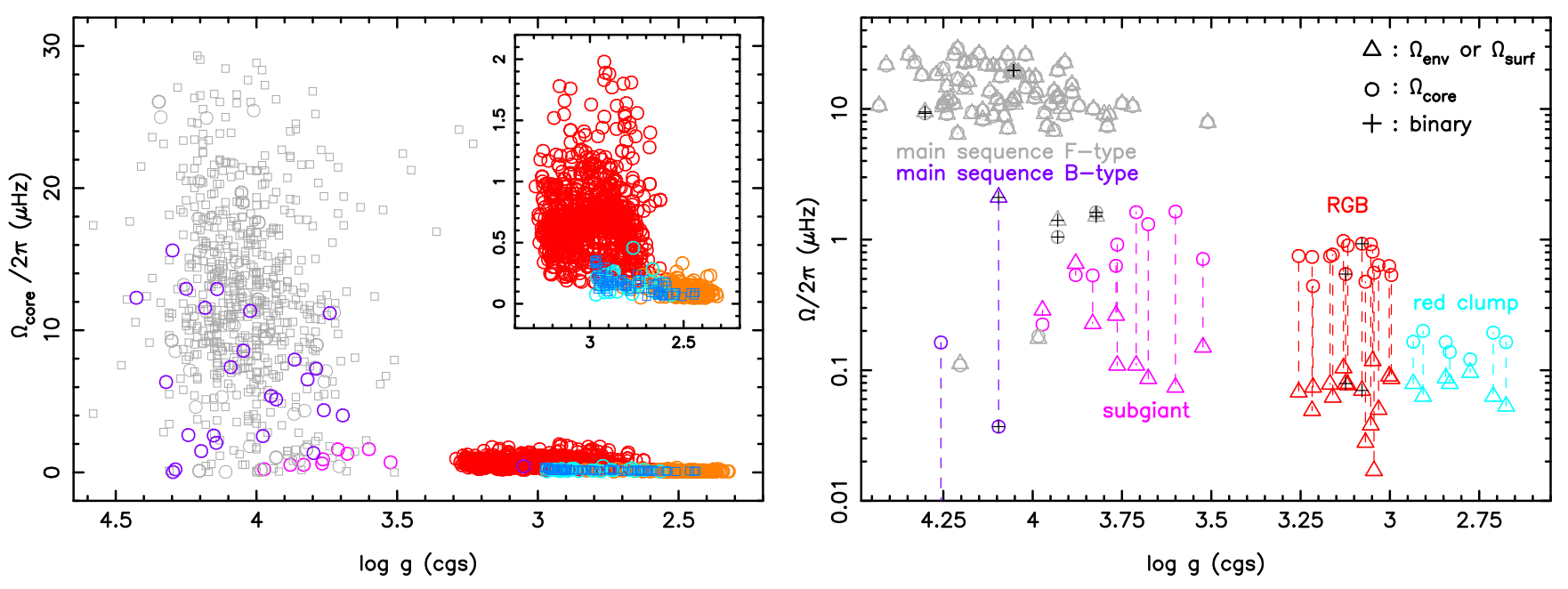

FIG. 6. Left panel: core (near-core) rotation rates derived from mixed (gravity) modes for stars in core-hydrogen burning (purple and gray), hydrogen-shell burning (pink and red), and core-helium burning (after the helium flash in orange and avoiding the helium flash in cyan). The circles indicate stars with asteroseismic estimates of $\Omega_{\text {core }}$ and $\log g$ taken from Aerts, Mathis, and Rogers (2019); their errors are smaller than the symbol size. The squares are additional stars with asteroseismic determinations for $\Omega_{\text {core }}$ but with less reliable values for $\log g$ from spectroscopy and/or stellar models from Li et al. (2020) (622 $\gamma$ Dor stars, indicated in gray) and from Tayar et al. (2019) (72 core-helium-burning red giants in blue). Their uncertainties for $\log g$ range from 0.2 (blue squares) to 0.5 dex (gray squares) and are omitted for clarity. Right panel: all stars with an additional measurement of the envelope (from $p$ modes) or surface (from rotational modulation) rotation frequency. Adapted from Aerts, Mathis, and Rogers, 2019.

(denoted as $\Omega_{\text {core }}$ throughout the review) in Fig. 6. We also highlight the envelope $\left(\Omega_{\text {env }}\right)$ or surface $\left(\Omega_{\text {surf }}\right)$ rotation frequency for the stars with this information. Figure 6 updates the work of Aerts, Mathis, and Rogers (2019), who presented these asteroseismic measurements for low- and intermediatemass stars distributed across all evolutionary stages from Kepler photometry. We discuss these results extensively in Sec. IV, but we point out here that almost all single stars in the covered mass range of $[0.8,3.3] M_{\odot}$ were found to rotate nearly uniformly during the core-hydrogen-burning and core-heliumburning phases and that the angular momentum of the heliumburning core of these stars is in agreement with the angular momentum of white dwarfs. Figure 6 implies a strong decrease of core angular momentum in the stars when stars have a convective core. Current stellar evolution theory of rotating stars cannot explain these asteroseismic results. This calls for a reevaluation of $1 \mathrm{D}$ models with rotation.

Stellar models with rotation often adopt the approximation of shellular rotation, following Zahn (1992). In this approximation, one assumes that the chemical composition and the angular velocity remain constant on isobars. As such, the ratio of the rotation frequency of the star $\Omega(r)$ with respect to $\Omega_{\text {crit }}$ [or the accompanying $v(r) / v_{\text {crit }}$ ] is used as input for the numerical computations of the stellar models. Given the limited knowledge on angular momentum evolution during the contraction phase, the input ratio $\Omega\left(R_{\star}\right) / \Omega_{\text {crit }}$ is usually taken at the ZAMS, assuming a rigid rotation profile to start the evolutionary computations.

From a theoretical perspective, rotation is expected to induce a myriad of processes and instabilities in the stellar interior, leading to transport of angular momentum and of chemical species. This was extensively discussed by Maeder (2009). As recently reviewed in the modern context of asteroseismology, these macroscopic processes can be classified into four main categories (Aerts, Mathis, and Rogers, 2019, Sec. 3): meridional circulation, hydrodynamical instabilities, magnetorotational instabilities, and IGWs. However, the concept of "rotational mixing" in stellar evolution computations and in the literature often stands for the macroscopic element transport due to the action of circulation and all instabilities together. Further, in analogy to rotational mixing, we will use the term "pulsational mixing" for element transport caused by waves. Because rotational or pulsational mixing is expected to homogenize the chemical mixture in the layers where they are active on short timescales, models including these ingredients often ignore the microscopic atomic diffusion effects leading to concentrations of species. However, there is no justified physical reason for this "computationally convenient" simplification when the timescales of these processes are similar; see Deal et al. (2020).

The transport equation controlling the evolution of the angular momentum $r^{2} \Omega(r)$ reads

$$
\begin{aligned}
\frac{\partial}{\partial t}\left(r^{2} \Omega\right)= & \frac{1}{5 \rho r^{2}} \frac{\partial}{\partial r}\left[\rho r^{4} \Omega U(r)\right] \\
& +\frac{1}{\rho r^{2}} \frac{\partial}{\partial r}\left(\rho r^{4} D_{\text {shear }} \frac{\partial \Omega}{\partial r}\right) .
\end{aligned}
$$

Here $U(r)$ is the radial component of the velocity due to meridional circulation and the diffusion coefficient $D_{\text {shear }}$ represents a variety of vertical shear instabilities occuring between layers subject to different velocities (Maeder, 2009). In addition to these instabilities, IGWs also occur in the radiative zones of stellar interiors. Given that the dominant restoring force for an $\mathrm{IGW}$ is the buoyancy force of Archimedes, the frequencies of IGWs are below $N(r)$. These IGWs propagate in the radiative zones of the star, 

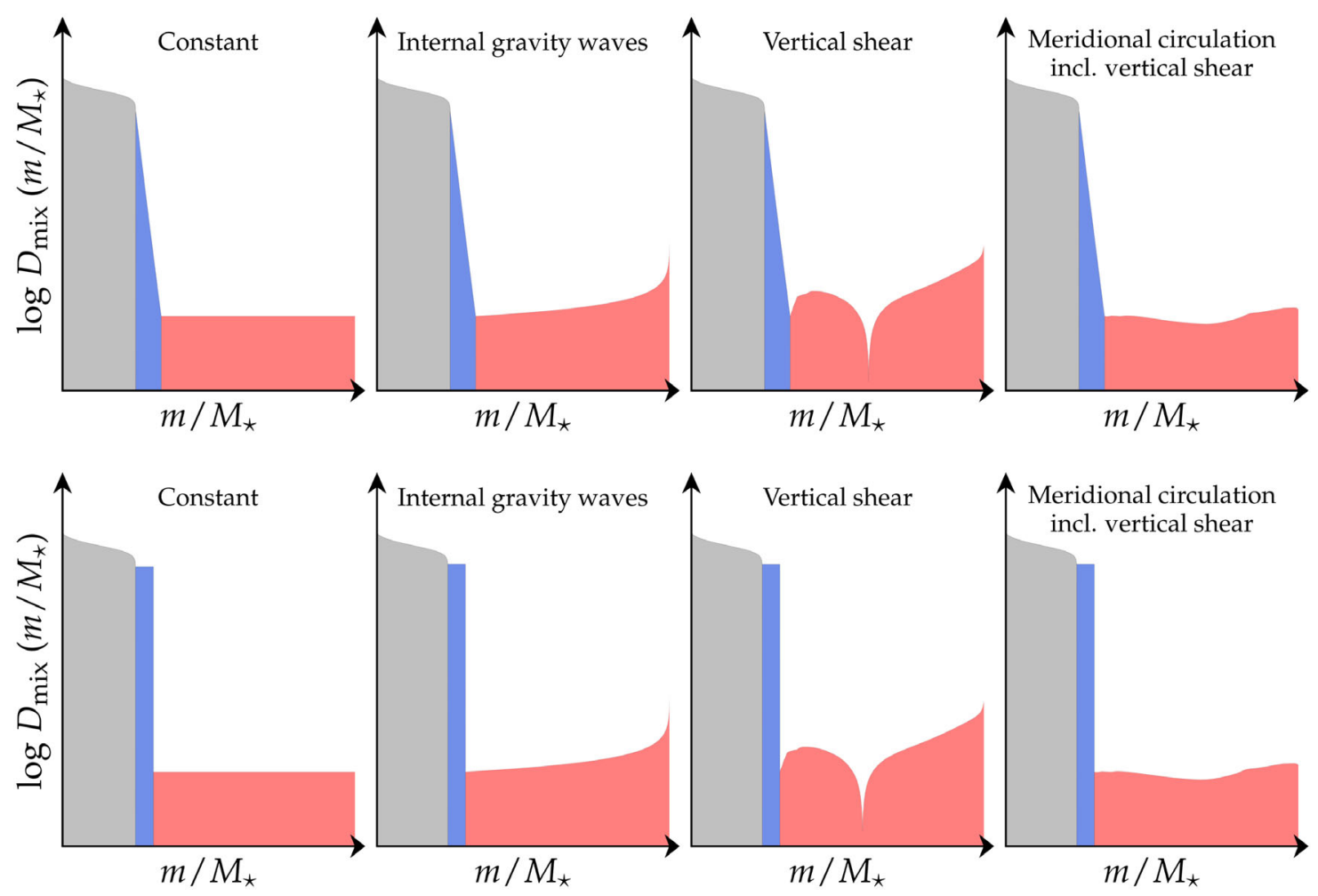

FIG. 7. Schematic representation of mixing profiles due to various transport processes in stars with a convective core (indicated in gray) and a radiative envelope for exponentially decaying diffusive core overshooting (upper panels) and convective penetration (lower panels) as CBM (purple). Four types of envelope mixing based on different theoretical frameworks are considered (pink), as labeled. Based on envelope mixing profiles for $5 M_{\odot}$ and $3 M_{\odot}$ ZAMS models computed by Georgy et al. (2013) and Rogers and McElwaine (2017), respectively. Adapted from Pedersen et al., 2021.

where they dissipate, depositing angular momentum efficiently in the layers where they break. A pioneering study of the excitation and propagation of IGWs in Sun-like stars was presented by Charbonnel and Talon (2005). It demonstrated the capacity of IGWs to transport angular momentum in an efficient way, explaining the flat rotation profile of the Sun derived from helioseismology.

As for the transport of the chemical species due to rotation, Chaboyer and Zahn (1992) showed that it can be approximated as a diffusive process in the presence of strong horizontal turbulence due to shear instabilities. For this reason, the diffusive part in the chemical composition equations in Eq. (27) gets extra terms due to various effects of rotation, each with its own diffusion coefficient (Maeder, 2009). Aside from rotation, additional causes of element mixing are also considered, particularly in transition layers that are stable against the Schwarzschild criterion, but unstable against the Ledoux criterion, for the cases of both $\nabla_{\mu}>0$ (called semiconvective mixing) and $\nabla_{\mu}<0$ (called thermohaline mixing). Magnetism and IGWs may also affect the mixing. Overall, this brings a multitude of extra diffusion coefficients that affect the chemical composition profiles of the star, aside from $D_{\text {conv }}(r)$ and $D_{\text {ov }}(r)$ included in Eq. (27). For rotation, these have been grouped as $D_{\text {shear }}(r)$ and $D_{\text {eff }}(r)$ adopting the notation by Maeder (2009), where the latter is due to meridional circulation in the approximation of strong horizontal turbulence and the former stands for the joint effect of vertical shear due to all sorts of rotational (and possibly magnetic) instabilities. Pulsational mixing profiles due to IGWs, adopting a diffusion approximation, were derived from hydrodynamical simulations for a $3 M_{\odot}$ ZAMS star by Rogers and McElwaine (2017), resulting in a diffusion coefficient depending on the density as $D_{\mathrm{IGW}}(r) \sim$ $D_{\text {IGW }} \rho^{-\gamma}(r)$ with $\gamma \in[0.5 ; 1]$.

Figure 7 offers a schematic representation of mixing profiles adopted in stellar evolution computations, where the envelope mixing profiles were stitched to the CBM at an arbitrary level. The two rightmost panels show profiles for a $5 M_{\odot}$ ZAMS model rotating at $50 \%$ of the critical rate taken from Georgy et al. (2013); the particular shape of the third panels from the left in Fig. 7 is due to the drop in $U(r) \simeq 0$ in the envelope layers near $m / M_{\star} \simeq 0.5$; cf. Maeder (2003). Similar sharp-peaked mixing profiles based on independently developed stellar evolution codes were found by Heger, Langer, and Woosley (2000), Chieffi and Limongi (2013), and Paxton et al. (2013), among others. The profile labeled "Internal gravity waves" is from a $3 M_{\odot}$ nonrotating model computed by Rogers and McElwaine (2017). In general, the mixing profiles indicated in Fig. 7 vary strongly during the evolution of the star, but it is poorly understood how. In this sense, none of these profiles are calibrated. Asteroseismology offers a major tool to infer the overall mixing profiles throughout stars, denoted as of now as $D_{\text {mix }}(r, t)$.

Inferences of the internal mixing in stars received less attention than the probing of $\Omega(r, t)$ thus far. The reason is simple: estimation of $D_{\text {mix }}(r, t)$ is much harder than of $\Omega(r, t)$. The latter can be achieved in a quasi-model-independent way 
and almost directly from the Fourier transform of the data, as explained in Sec. II.B. Given that the levels of $D_{\text {mix }}(r, t)$ as displayed in Fig. 7 differ by orders of magnitude in the literature, it is highly beneficial to infer asteroseismic levels of mixing (and the accompanying convective core mass) to bring the models into agreement with measurements of nonradial oscillations of intermediate- and high-mass stars. Asteroseismology of $\gamma$ Dor, SPB, and $\beta$ Cep stars has the potential to provide the answer if proper ensembles of such pulsators are subjected to asteroseismic inference. We return to this potential and its first applications in Sec. IV.

\section{One-dimensional equilibrium models as input for asteroseismology}

For stars with detected nonradial oscillations, space asteroseismology brings an entirely new way to assess the rotation frequency $\Omega(r, t)$ and the overall chemical mixing $D_{\text {mix }}(r, t)$ in the radiative zones of stars. Indeed, mode frequencies provide high-precision observational constraints coming directly from the deep stellar interior. Assembling asteroseismic data for stars in various evolutionary stages allows one, in principle, to assess the change of $\Omega(r)$ and $D_{\text {mix }}(r)$ as a function of stellar age. Yet asteroseismic probing capacities for $\Omega(r, t)$ and $D_{\text {mix }}(r, t)$ are different for low-mass stars with a radiative core and a convective envelope than for high-mass stars with a convective core and a radiative envelope. They also differ for young stars burning hydrogen in their core and for old stars close to their final fate as stellar remnant. To understand why, it is necessary to dive into the nature of nonradial oscillations based on 1D equilibrium models.

As discussed earlier, the simplest versions of 1D stellar equilibrium models are nonrotating nonmagnetic models having only six free parameters for fixed choices of the input physics: the stellar birth mass $M_{\star}$, the initial chemical composition guided by a galactic enrichment law [such as the one given by Verma et al. (2019)] and expressed as relative mass fractions $\left(X_{\text {ini }}, Y_{\text {ini }}\right)$ (or equivalently $\left.X_{\text {ini }}, Z_{\text {ini }}\right)$, the mixing-length value $\alpha_{\mathrm{mlt}}$ that gives rise to the mixing profile $D_{\text {conv }}(r)$, the convective overshoot length scale $\alpha_{\text {ov }}$ that leads to the CBM profile $D_{\mathrm{ov}}(r)$, and the age $\tau$. Asteroseismic modeling will then consist of determining the maximum likelihood estimators (MLEs) of these six free parameters from measured oscillation mode frequencies (often accompanied by other observables). For a rotating star, at least one additional parameter has to be added ( $\Omega$ for the simplest case of rigid rotation). Once the most likely 6D or 7D parameter vector $\theta \equiv\left(M_{\star}, X_{\mathrm{ini}}, Y_{\mathrm{ini}}, \alpha_{\mathrm{mlt}}, \alpha_{\mathrm{ov}}, \tau\right)$ has been found, the exercise can be repeated for other choices of the input physics to come to an overall selection of the best stellar models for an ensemble of stars. Any residual values between the measured and theoretically predicted oscillation frequencies of unambiguously identified modes can then be exploited to assess shortcomings in $\Omega(r, \tau)$ and $D_{\text {mix }}(r, \tau)$ for the fixed chosen input physics. Once a sufficiently large and unbiased (in terms of rotation, initial chemical composition, etc.) sample of nonradial pulsators with suitable modes is available from observations, we can investigate whether they adhere to the same theory of stellar structure and evolution or instead need different internal mixing profiles as in Fig. 7. We return to this procedure of "ensemble asteroseismology" and discuss simplifications and applications of it for various types of stars in Sec. IV. An overall scheme representing this approach is graphically visualized in Fig. 16 and is discussed in Sec. III.

\section{B. Linear nonradial oscillation modes}

We now consider small perturbations to $1 \mathrm{D}$ spherically symmetric stellar models in hydrostatic equilibrium, whose quantities we assume to have been derived from solving the stellar structure equations. We denote the equilibrium solutions at age $\tau$ as $m_{0}(r), p_{0}(r), L_{0}(r), T_{0}(r), X_{i, 0}(r)$. We assume that the oscillations cause $3 \mathrm{D}$ periodic deviations from equilibrium with amplitudes that justify a linear approach in the derivation of the pulsation equations. In practice this implies that we perturb Eqs. (18), (19), and (21) while retaining only the linear terms in the perturbations. For example, a fluid element at position vector $\boldsymbol{r}_{0}$ in the equilibrium model of the star is displaced due to the 3D stellar oscillations to the vector $\boldsymbol{r}_{0}+\boldsymbol{\delta} \boldsymbol{r}$, where $\delta \boldsymbol{r}$ is the Lagrangian perturbation of the position vector. The Lagrangian perturbation to the pressure then becomes

$\delta p(\boldsymbol{r})=p\left(\boldsymbol{r}_{0}+\delta \boldsymbol{r}\right)-p_{0}\left(\boldsymbol{r}_{0}\right)=p\left(\boldsymbol{r}_{0}\right)+\boldsymbol{\delta} \boldsymbol{r} \cdot \nabla p_{0}-p_{0}\left(\boldsymbol{r}_{0}\right)$.

All perturbed quantities that occur in Eqs. (18), (19), and (21) can be deduced in a similar way. The linearized versions of the perturbed equations are obtained by inserting expressions like Eq. (33) into the full equations, subtracting the version of those equations for the static equilibrium solutions, and neglecting all terms of order higher than 1 in the perturbed quantities. Aerts, Christensen-Dalsgaard, and Kurtz (2010) gave full derivations in Chap. 3; we adopt the notations from that book. Additional extensive discussions on the theory of nonradial oscillations were given by Cox (1980), Unno et al. (1989), and Smeyers and Van Hoolst (2010), where the last work includes a particularly extensive historical perspective of the topic.

We argued in Sec. II.A that it is meaningful to ignore the nonradial components of the centrifugal force for stars that rotate up to $\sim 70 \%$ of their critical rotation frequency and to treat the Coriolis and Lorentz forces only at the level of the 3D perturbations to computed nonradial g modes, but not for the equilibrium models. For $\mathrm{p}$ modes, this validity already breaks down above $\sim 15 \%$. In the following sections, we gradually build up the complexity of the treatment of the oscillations. An obvious simplification occurs when we consider the adiabatic approximation for the computation of the modes. This means that we can ignore the perturbations of the entropy $S$ in Eq. (21). We do so in the rest of this section. Working in the adiabatic approximation is good and fully justified as long as we consider modes that are mostly sensitive to the physics in the deep stellar interior where adiabaticity is well met. This restriction is a point of attention when dealing with modes that have their dominant energy in the envelope of the star, close to the stellar surface; cf. p modes in low-mass stars as in Fig. 9, which is discussed later. 


\section{Pressure and gravity modes}

We simplify the perturbed stellar structure equations maximally by ignoring the Lorentz and Coriolis forces. In that case, the only forces at play are the pressure force and gravity. These simplifications offer maximal separability in terms of spherical polar coordinates $(r, \theta, \phi)$ and time $t$, where $r$ is the distance to the center of the star, $\theta$ is the angle from the polar axis, which is taken to coincide with the rotation axis of the star, and $\phi$ is the longitude. The displacement $\delta r$ can then be separated into radial and horizontal components as

$$
\boldsymbol{\delta} \boldsymbol{r}=\xi_{r} \boldsymbol{a}_{r}+\boldsymbol{\xi}_{h}
$$

where $\boldsymbol{a}_{r}$ is a unit vector directed radially outward. The solutions to the resulting perturbed versions of the equations, along with proper boundary conditions for the center and for the surface of the star [not discussed here; see Unno et al. (1989)], lead to nontrivial solutions only for the nonzero eigenfrequencies $\omega$ of the stellar equilibrium model. Each of these eigenfrequencies corresponds to a so-called time-dependent spheroidal mode of oscillation. Because the equations are homogeneous, the eigensolutions are determined only up to a constant factor.

Each of the nonradial eigenmodes of the equilibrium model corresponds to a displacement vector $\boldsymbol{\xi}$ whose components are written in terms of a mode degree $l$, azimuthal order $m$, and radial order $\quad n \quad$ as $\boldsymbol{\xi}(r, \theta, \phi, t)=\left[\left(\xi_{r, n l} \boldsymbol{a}_{r}+\xi_{h, n l} \nabla_{h}\right) Y_{l}^{m}(\theta, \phi)\right] \times$ $\exp \left(-i \omega_{n l m} t\right)$. Modes with $m=0$ are called axisymmetric (or zonal) modes; these reveal $l$ latitudinal surface nodal lines. For $|m|=l$, all surface nodal lines are lines of longitude. These modes are called sectoral modes. Modes with $0 \neq|m|<l$ are called tesseral modes and have $|m|$ longitudinal and $l-|m|$ latitudinal nodal lines. As a special case, radial oscillations have $l=0$; i.e., they do not reveal any nodal lines on the stellar surface. The angular dependence of the radial eigenvector component $\left(\xi_{r}\right)$ of some nonradial modes was graphically illustrated in Fig. 2. Space photometry has predominantly given rise to the detection of low-degree modes, typically with $l<4$. As discussed in Fig. 2, the higher the degree of the mode, the more the detection is prone to partial cancellation due to the integration of the mode's overall perturbation over the visible stellar surface in the line of sight. The cancellation gets more pronounced as $l$ increases, because more and smaller patches with opposite sign occur in the spherical harmonic $Y_{l}^{m}$ that represents $\xi_{r}$. The level of cancellation also depends on the angle between the rotation axis and the line of sight (chosen as $60^{\circ}$ in Fig. 2). The radial order of the mode $n$ represents the number of nodes of $\xi_{r}$ in the stellar interior, where these nodes are counted positively for $\mathrm{p}$ modes and negatively for $g$ modes. For unevolved stars, the assignment of $n$ is straightforward in that modes with $n=0$ have no nodes aside from the stellar center. These modes are called fundamental modes, abbreviated as "f modes." For mixed modes in evolved stars, however, one could obtain $n=0$ from the occurrence of pairs of nodes for $\xi_{r}$ in the p- and g-mode cavities. Since the assignment of the radial order $n$ is used to classify modes, such a classification may be subject to complex issues, as explained in Sec. 3.5.2 given by Aerts, Christensen-Dalsgaard, and Kurtz (2010), to which we refer for additional details. Further, we adopt the convention that the sign of $m$ distinguishes prograde $(m>0)$ from retrograde modes $(m<0)$, where the former represent motions along the rotation of the star and the latter represent motions against it.

The general system of differential equations that lies at the basis of the eigenvalue problem describing nonradial oscillation modes is of fourth order in the unknown perturbed quantities, which are $\xi_{r}$ and the perturbations to the pressure $\delta p$, gravitational potential $\delta \Phi$, and the derivative of $\delta \Phi$. These equations have $Y_{l}^{m}(\theta, \phi) \exp \left(-\mathrm{i} \omega_{n l m} t\right)$ as a common factor. Hence this factor can be divided out. The resulting ordinary differential equations to solve for the radial component of the unknown eigenfunctions do not depend on the azimuthal order $m$ due to the assumption of having a spherically symmetric equilibrium model. This fourth-order system of equations needs four boundary conditions to be solved. However, it is often appropriate to ignore the perturbation to the gravitational potential because this perturbation is sufficiently small relative to the perturbation to the density. This is known as the Cowling approximation (Cowling, 1941). It renders the system of equations to second order and thus requires only two boundary conditions to get physically meaningful solutions. These are $\xi_{r} \simeq l \xi_{h} \sim r^{l-1}$ for $r \rightarrow 0$ and $\delta p=0$ for $r \rightarrow R_{\star}$ (Unno et al., 1989). This also allows for the derivation of an analytical expression for the ratio of the horizontal to the radial displacement at the stellar surface, which depends only on the frequency of the mode

$$
\frac{\xi_{h}\left(R_{\star}\right)}{\xi_{r}\left(R_{\star}\right)} \simeq \frac{G M_{\star}}{\omega_{n l 0}^{2} R_{\star}^{3}} .
$$

This ratio is called the " $\mathrm{K}$ value" by observers. Typical values for this ratio are below 0.001 for high-order p modes as in the Sun and 10-1000 for high-order g modes of core-hydrogenburning stars. Mathematically, the Cowling approximation is valid only for modes of high radial order $n$ and of "high" degree $l$. One should therefore not expect this to be an optimal approximation for low-order low-degree modes, and, in particular, not for $l=1, n=0 \mathrm{f}$ modes; see Sec. 3.4.1 given by Aerts, Christensen-Dalsgaard, and Kurtz (2010). Hence, observed stars may reveal frequency values for their $\mathrm{f}$ modes that do not coincide with those computed in the Cowling approximation.

The two pulsation equations resulting from adoption of the Cowling approximation can be combined into a single approximative second-order differential equation for $\xi_{r}$ as follows:

$$
\frac{\mathrm{d}^{2} \xi_{r}}{\mathrm{~d} r^{2}} \simeq \frac{\omega^{2}}{c_{\mathrm{s}}^{2}}\left(1-\frac{N^{2}}{\omega^{2}}\right)\left(\frac{S_{l}^{2}}{\omega^{2}}-1\right) \xi_{r}
$$

with $N(r)$ as given in Eq. (26) and where we have introduced the following local characteristic acoustic frequency (also called the Lamb frequency) for the mode with degree $l$ :

$$
S_{l}^{2}(r) \equiv \frac{l(l+1) c_{\mathrm{s}}^{2}}{r^{2}}
$$

with $c_{\mathrm{s}}$ the sound speed in the stellar interior. While Eq. (36) is the simplest form in which nonradial oscillations can be 

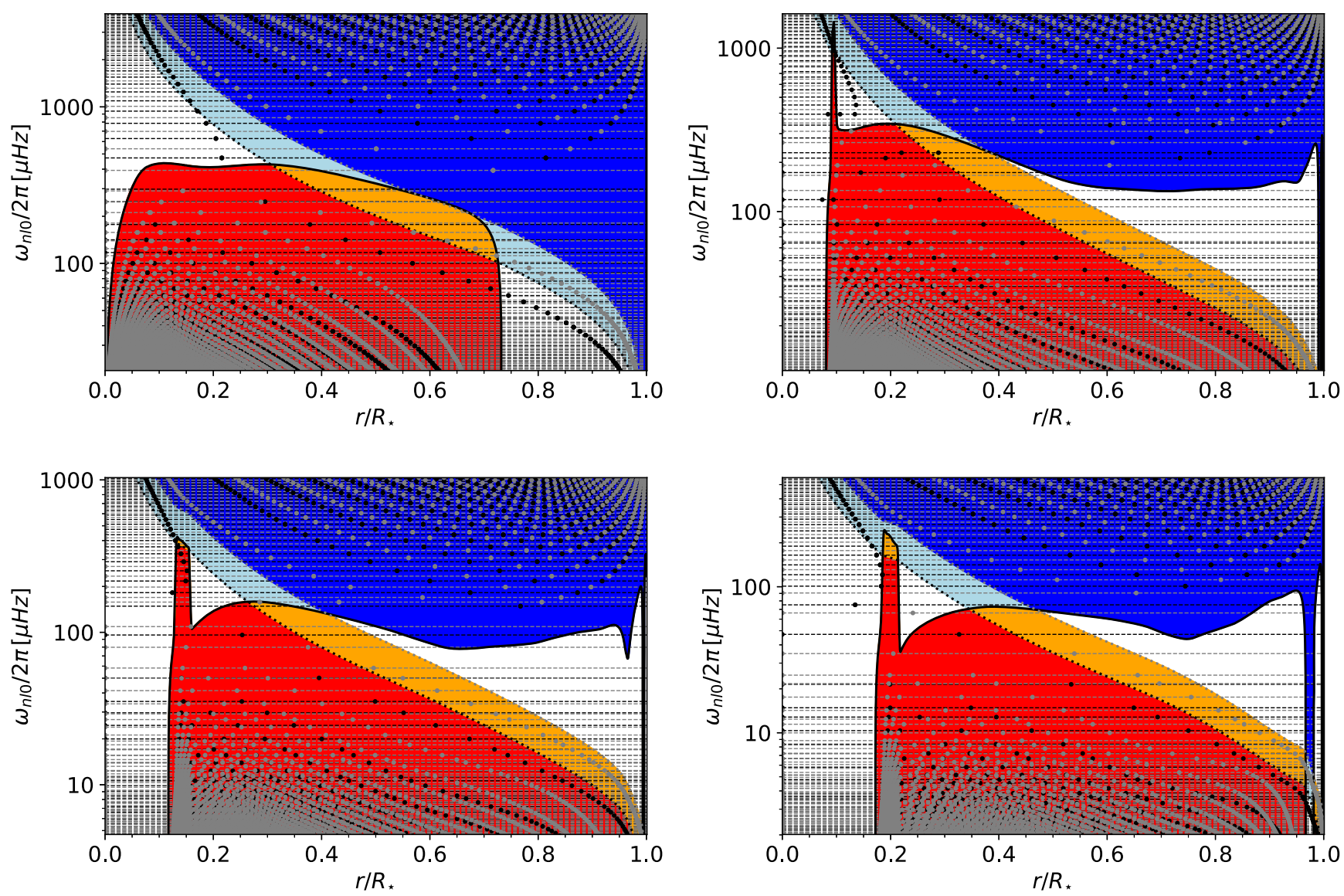

FIG. 8. Propagation diagrams showing the mode cavities of axisymmetric $\mathrm{p}$ and $\mathrm{g}$ modes in four stellar models halfway through the core-hydrogen-burning stage of evolution. The models have masses of $1 M_{\odot}, 1.7 M_{\odot}, 5 M_{\odot}$, and $15 M_{\odot}$ from top left to bottom right. The thick solid black line indicates $N(r)$, while the dotted black and gray lines represent $S_{1}(r)$ and $S_{2}(r)$, respectively. The values of the dipole (quadrupole) mode frequencies are indicated as black (gray) horizontal lines. The position of the nodes of $\xi_{r}$ are indicated as thick black and gray dots for $l=1$ and 2, respectively. The red region is the g-mode cavity for dipole modes; it is extended by the orange part for quadrupole modes. The dark blue region is the mode cavity of quadrupole $(l=2) \mathrm{p}$ modes. It is extended by the light blue region for dipole $(l=1) \mathrm{p}$ modes. The modes correspond to evanescent waves in the white regions in the stellar envelope. The $g$ modes cannot propagate in the convective core of the three most massive stellar models, nor in the outer $\sim 26 \%$ of the convective envelope of the $1 M_{\odot}$ model, where $N^{2}(r)<0$. Partially based on models from Mombarg et al. (2019).

described, it still leads to a good approximation for the mode frequencies, and more importantly to insightful interpretations and an elegant way to introduce the so-called mode cavities. These are illustrative when plotted in propagation diagrams. Solutions for $\xi_{r}$ from solving Eq. (36) are oscillatory as a function of $r$ when (a) $|\omega|>|N|$ and $|\omega|>S_{l}$ or when (b) $|\omega|<|N|$ and $|\omega|<S_{l}$. The positions inside the star where these conditions are met correspond with the zones in the stellar interior where the modes resonate inside a cavity. In this sense, the modes correspond to standing waves in their mode cavity and are said to be trapped there. The modes that meet conditions (a) are dominantly restored by the pressure force and are therefore called pressure modes, usually labeled as $\mathrm{p}$ modes. Within their mode cavity, these modes are resonating sound waves (also called acoustic waves). By convention, we denote the number of nodes in the stellar interior as $n>0$. Buoyancy is the dominant restoring force when conditions (b) are met, and these modes are therefore called gravity modes, labeled as g modes. Their radial order is denoted by $n<0$, which means that they have $-n>0$ nodes in the interior of the star. Within their mode cavity, they behave like low-frequency (i.e., slow) internal gravity waves with a dominant horizontal displacement in a gas that is radially stratified due to gravity. Finally, solutions for $\xi_{r}$ when solving Eq. (36) have an exponential behavior when $|N|<$ $|\omega|<S_{l}$ or $S_{l}<|\omega|<|N|$. The zones in which the modes behave exponentially are called evanescent regions, and the eigensolutions decrease or increase exponentially the farther away they are from the mode cavities.

Figure 8 shows propagation diagrams for four stellar models that represent stars about halfway through their core-hydrogenburning stage, with birth masses of $1 M_{\odot}, 1.7 M_{\odot}, 5 M_{\odot}$, and $15 M_{\odot}$ and with solar chemical composition and mixture. The oscillation modes were computed with the open source pulsation code GYRE (Townsend and Teitler, 2013; Townsend, Goldstein, and Zweibel, 2018), coupled to equilibrium models computed with the open source code Modules for Experiments in Stellar Astrophysics (MESA) (Paxton et al., 2011, 2013, 2015, 2018, 2019). The results in Fig. 8 were obtained not by relying on the Cowling approximation but rather by solving the fourth-order set of equations, as in modern applications of asteroseismology. The mode cavities for 

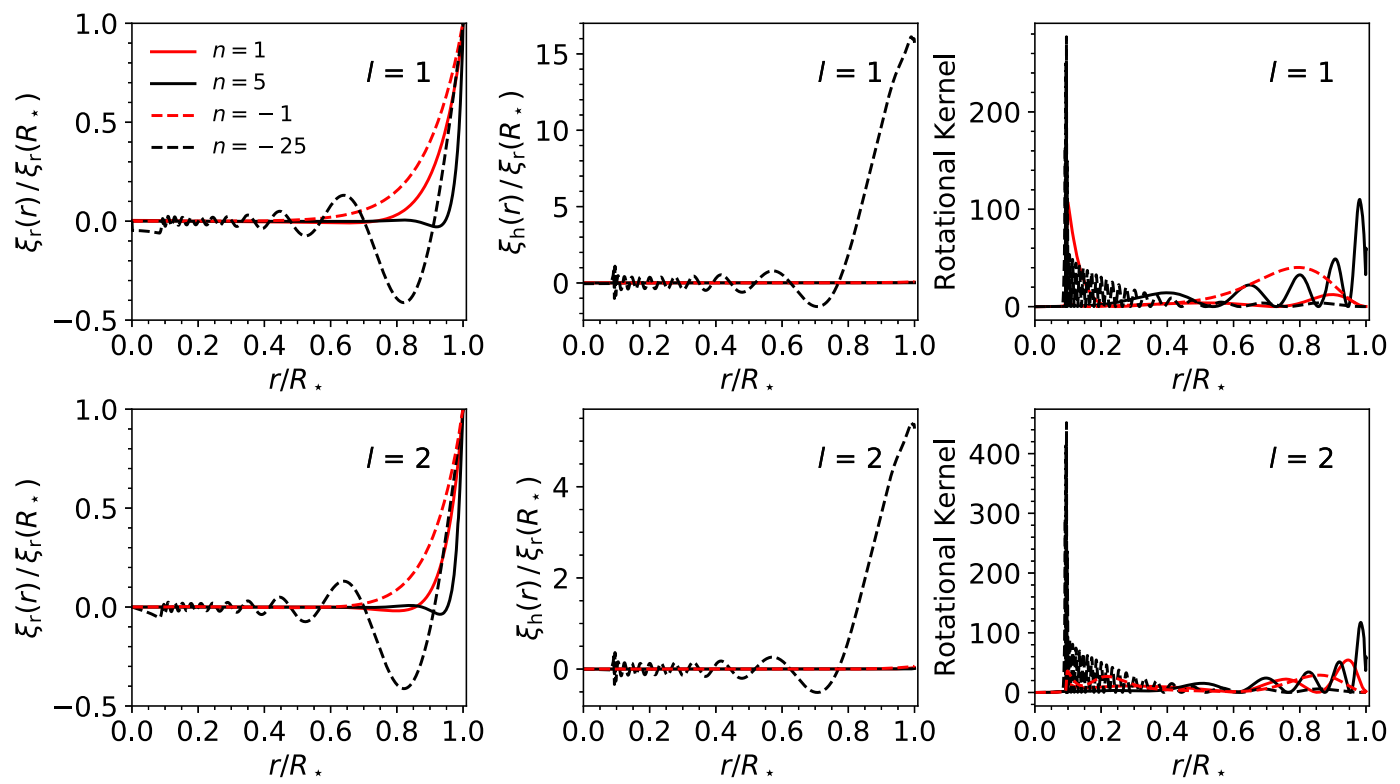

FIG. 9. Radial (left panels) and horizontal components (middle panels) of the Lagrangian displacement of four indicated axisymmetric $(m=0) \mathrm{p}$ and g modes for $l=1$ (top panels) and $l=2$ (bottom panels) of a stellar model with $M_{\star}=1.7 M_{\odot}$ halfway through its corehydrogen-burning stage of evolution. The right panels show the rotation kernel defined by Eq. (45), which represents the probing power of an oscillation mode. Partially based on pulsation computations from Mombarg et al. (2020).

axisymmetric dipole $(l=1)$ and quadrupole modes $(l=2)$ are indicated, as are the mode's eigenvalues (horizontal lines) and positions of the radial nodes (dots). The importance of the receding convective core and the accompanying shape of $N(r)$ for the g-mode oscillations in intermediate- and high-mass models is visible in the lower panels of Fig. 8.

Eigenfunctions for eight modes are shown in the left and middle panels of Fig. 9 for the stellar model whose mode cavities are displayed in the upper right panel of Fig. 8. In the absence of predictive power for the mode amplitudes, we normalize the modes such that $\xi_{r}\left(R_{\star}\right)=1$ in Fig. 9. It can be seen by comparing the left and middle panels that high-order $\mathrm{g}$ modes have dominant horizontal displacements, while it is the opposite for p modes. This is in line with the predictions based on the Cowling approximation. It is a general property of $\mathrm{p}$ and $g$ modes. Moreover, Fig. 9 shows that $\mathrm{p}$ modes have higher amplitudes in the outer stellar envelope than in the inner regions, while g modes have their highest amplitude in the regions near the convective core.

The mode cavities change as a star evolves, reflecting the increased density contrast in the stellar interior. This drastically changes the profile of the sound speed $c_{\mathrm{s}}(r)$, and hence the profile of $S_{l}(r)$ as well. As a result, the p-mode cavities decrease in frequency and the evanescent zones become narrower. Their exponential decay may hence be limited, allowing them to reach the g-mode cavity and couple to the eigenfrequencies of the $\mathrm{g}$ modes. Such is the case for dipole modes in red-giant stars. These modes are therefore called mixed modes: they have a p-mode character in the outer envelope and a g-mode character in the inner regions of the star. We refer to Figs. 2-4 in the Supplemental Material given by Aerts, Mathis, and Rogers (2019) for propagation diagrams of mixed modes in red-giant stars and refrain from repeating such diagrams here for brevity. This mixed character of these dipole modes was predicted theoretically by Dziembowski
(1971) and Shibahashi (1979). Dupret et al. (2009) pointed out their probing power for the center of evolved stars following the discovery of nonradial oscillations in red giants from CoRoT (De Ridder et al., 2009) prior to their actual detection in Kepler data. We return to this capacity in Sec. IV.

\section{Asymptotic representations of high-order modes}

As discussed in Sec. III, mode identification is a critical step to be taken before any asteroseismic inference can be made. Indeed, a comparison between the detected and theoretically computed oscillation mode frequencies $\omega_{n l m}$ can be made only after the mode labels $(n, l, m)$ have been derived. Given that we cannot resolve the surfaces of pulsating stars in sufficient detail (except for the Sun), we cannot identify the spherical wave numbers $(l, m)$ of the nonradial modes from maps of the eigenfunctions, as in the graphical representation in Fig. 2. We somehow have to derive the mode identification from the observables. To this end, asymptotic representations of high-order modes help a great deal, although other, more empirical methods for mode identification of modes exist as well; see Chap. 6 given by Aerts, Christensen-Dalsgaard, and Kurtz (2010). Here we limit the discussion to mode identification based on patterns deduced among the detected oscillation mode frequencies or mode periods.

The asymptotic theory of nonradial oscillations is based on second-order differential equations describing the modes, which illustrates again why the Cowling approximation is so useful for asteroseismology. The convenience of asymptotic representations of high-order modes was initially considered for the case of linear radial modes by Ledoux (1962) (in French). He recognized that the radial-mode properties can be derived from a second-order differential equation, which constitutes a Sturm-Liouville eigenvalue problem with singular end points at $r=0$ and $r=R_{\star}$. The asymptotic 
properties of nonradial oscillation modes have been studied more generally ever since and are well covered in the literature, at various levels of mathematical detail. See the extensive papers by Tassoul (1980, 1990), and see Sec. III.4 and Appendix E given by Aerts, Christensen-Dalsgaard, and Kurtz (2010) for a general background and results. Smeyers and Van Hoolst (2010) provided in their Chaps. 14-18 thorough mathematical details and comparisons for the different regimes of validity while considering different types of modes and various types of equilibrium models.

High-order $\mathrm{p}$ modes.-We first consider the case of low-degree high-order axisymmetric p modes. To leading order in the asymptotics, the frequencies of such modes comply with

$$
\nu_{n l} \equiv \frac{\omega_{n l}}{2 \pi} \simeq\left(n+\frac{l}{2}+\frac{1}{4}+\alpha\right) \Delta \nu
$$

where we have dropped the $m=0$ wave number in the notation and where we use the cyclic frequencies of the oscillation modes. In Eq. (38)

$$
\Delta \nu=\left(2 \int_{0}^{R} \frac{\mathrm{d} r}{c_{\mathrm{s}}}\right)^{-1}
$$

is called the large frequency separation. It is the inverse of twice the sound travel time between the center and the surface of the star. On the basis of this theoretical prediction, one expects the frequencies of the p modes with sufficiently high $n$ to be equally spaced and modes with the same value of $n+l / 2$ to have almost the same frequency values, since $\nu_{n l} \simeq \nu_{n-1 l+2}$. Such frequency patterns have indeed been observed for the solar low-degree p modes and these observational findings have given rise to the research field of helioseismology; see Christensen-Dalsgaard (2002) for an extensive review, including historical aspects of the development of asteroseismology of "our own" star.

Given that the excitation and damping of the solar oscillations is due to the turbulent convection in its outer envelope, we expect similar asymptotic behavior for the high-order $\mathrm{p}$ modes of all stars with a convective envelope. This was confirmed almost two decades ago from ground-based velocity data for $\beta$ Hydri (Bedding et al., 2001) and $\alpha$ Cen A (Bouchy and Carrier, 2001), and prior to space photometry for several tens of stars (Aerts, Christensen-Dalsgaard, and Kurtz, 2010). Space photometry confirms that stars with a convective envelope comply with asymptotic theory, from dwarfs to the bottom of the asymptotic giant branch. One of the best datasets of solarlike oscillations in a star other than the Sun was assembled for $16 \mathrm{Cyg} \mathrm{A}$; its PD spectrum in Fig. 10 illustrates the validity of the asymptotic theory.

Equation (38) is based on the dominant term in the asymptotic representation of low-order $p$ modes. The second-dominant term in the expansion leads to the so-called small frequency separation, given by

$$
\delta \nu_{n l} \equiv \nu_{n l}-\nu_{n-1 l+2} \simeq-(4 l+6) \frac{\Delta \nu}{4 \pi^{2} \nu_{n l}} \int_{0}^{R} \frac{\mathrm{d} c_{\mathrm{s}}}{\mathrm{d} r} \frac{\mathrm{d} r}{r},
$$

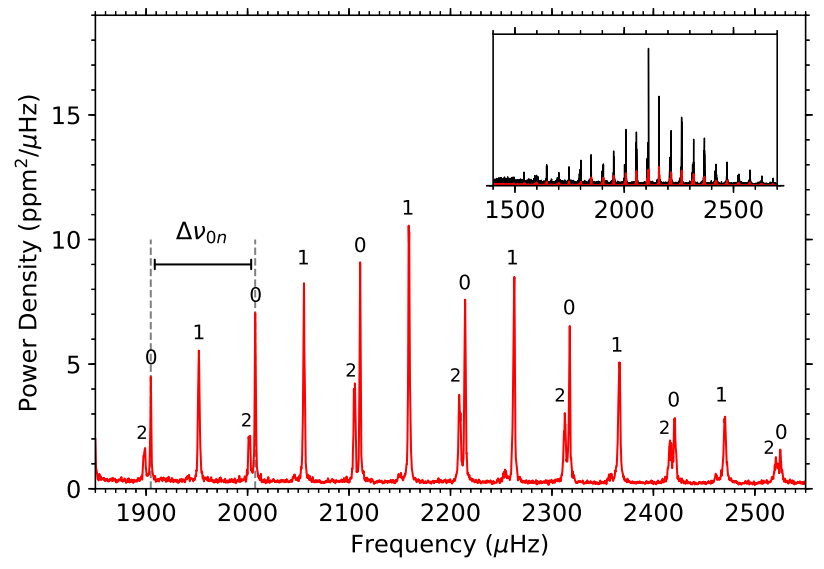

FIG. 10. Enlargement (red) of the observed envelope of oscillation signal revealed by the power density spectrum (black) of the solar analog $16 \mathrm{Cyg} \mathrm{A}$ as deduced from data assembled with the Kepler satellite. The $p$ modes are labeled by their degree $l$. The large frequency separation based on the detected radial mode frequencies is indicated. Adapted from Chaplin and Miglio, 2013.

where $c_{\mathrm{s}}\left(R_{\star}\right) \simeq 0$ was assumed to arrive at this approximation. From this expression, it is clear that $\delta \nu_{n l}$ probes the soundspeed gradient in the deep stellar interior. For stars in the corehydrogen-burning stage, $d c_{\mathrm{s}} / d r$ is highly sensitive to the hydrogen and helium composition profiles, which are directly impacted by the nuclear fusion. It is then readily understood that $\delta \nu_{n l}$ is of major diagnostic value to estimate the age of the exoplanet host star by comparing its observed values with predictions of this quantity based on equilibrium models. In the case of 16 Cyg A, as can be seen in Fig. 10, $\delta \nu_{n l}$ can be measured with high precision from the radial and quadrupole modes. This, and more sophisticated diagnostics for additional modes, was used by Bellinger et al. (2017) to find an asteroseismic estimate of $\tau=6.9 \pm 0.4$ Gyr. This is in excellent agreement with other methods for this well-characterized bright exoplanet host binary (Maia et al., 2019).

The similarity of the nonradial oscillations of $16 \mathrm{Cyg}$ A to those of the Sun as illustrated in Fig. 10 is representative of low-mass dwarfs with convective envelopes. This observational finding is of key diagnostic importance to estimate stellar masses, radii, and ages of such stars, as we discuss further in Sec. IV.

High-order g modes.-For high-order low-degree axisymmetric g modes, $\omega \ll N$ over most of the mode cavity; cf. Fig. 8. We denote with $r_{1}$ and $r_{2}$ the inner and outer positions of the g-mode cavity. In this case, the asymptotic analysis based on the Cowling approximation by Tassoul (1980) led to

$$
P_{n l}=\frac{\Pi_{0}}{\sqrt{l(l+1)}}\left(|n|+\alpha_{1, \mathrm{~g}}\right),
$$

where

$$
\Pi_{0} \equiv 2 \pi^{2}\left(\int_{r_{1}}^{r_{2}} N \frac{\mathrm{d} r}{r}\right)^{-1} .
$$

The quantity $\Pi_{0}$ stands for the buoyancy travel time and represents a characteristic period for the g modes of the star 
(as inverse of a frequency, it is expressed in the unit of time). In this case, the mode periods are asymptotically equally spaced in the order of the mode and the periodspacing value decreases with increasing $l$. The phase term $\alpha_{\mathrm{l}, \mathrm{g}}$ depends on whether the star has a radiative or a convective core.

Long before space asteroseismology, g-mode period-spacing patterns have been extensively exploited for pulsating white dwarfs, based on photometric data assembled from the Whole Earth Telescope (Winget et al., 1991, 1994). The short periods of their g modes (a few to tens of minutes) imply beating patterns in the light curves of only a few days, while the modes may have high amplitudes of percentage level. This led to the detection of tens of dipole and quadrupole modes that are subject to strong mode trapping in the outer thin $\mathrm{H}$ and He layers of these objects, where $N(r)$ experiences spikes due to strong changes in $\mu$. In the context of white dwarfs, polytropes can be taken as equilibrium models, leading to analytical expressions for $\alpha_{1, q}$ that allow detailed interpretation of the mode trapping in terms of the chemical composition and mass of the outer layers of such pulsators; see Brassard et al. (1992) for a seminal paper. Ground-based asteroseismology was therefore already highly successful for white dwarfs in the early 1990s.

For applications to SPB stars and $\gamma$ Dor stars, which are both core-hydrogen-burning g-mode pulsators with a convective core (cf. Figs. 1 and 8), $\alpha_{1, g}=\alpha_{\mathrm{g}}$ turns out to be independent of the mode degree $l$ and one gets $\Delta P \equiv P_{n l}-P_{n-1 l}=$ $\Pi_{0} / \sqrt{l(l+1)}$. Smeyers and Moya (2007) provided more sophisticated asymptotic analyses based on the full fourthorder theory of nonradial oscillations; i.e., they omitted the Cowling approximation. They developed the asymptotic approximations for both the cases of a radiative (SPB stars) and a convective ( $\gamma$ Dor stars) envelope. Although they derived more complicated expressions, the patterns to be expected from observations are well captured by Eq. (41). However, the number of nodes may differ by 1 compared with the simpler treatment from Tassoul (1980) based on the Cowling approximation. This implies that one should consider an uncertainty in the assignment of the radial order $n$ of at least 1 in any practical asteroseismic modeling based on observed g-mode period spacings. In general, modern pulsation codes usually adopt the Takata (2012) classification scheme to assign the radial order $n$ to modes.

The period-spacing pattern of high-order g modes offers a direct probe of the physical conditions in the region near the convective core of main-sequence stars. This offers interesting applications to assess the mixing at the bottom of the radiative envelope of core-hydrogen-burning stars, which is one of the major uncertainties in the theory of stellar evolution, as discussed in Sec. II.A. A seminal paper on this probing capacity was written by Miglio et al. (2008). In retrospect, this paper offered a preview of the major insights to come from $\mathrm{g}$ mode space asteroseismology when turned into practice. Aside from the somewhat controversial detection and interpretation of $\mathrm{g}$ modes in the Sun (García et al., 2007), the first g-mode period-spacing pattern detection for an intermediatemass dwarf came from CoRoT data of the $\sim 7 M_{\odot} \mathrm{B} 3 \mathrm{~V}$ star HD 50230. Eight axisymmetric g modes with consecutive

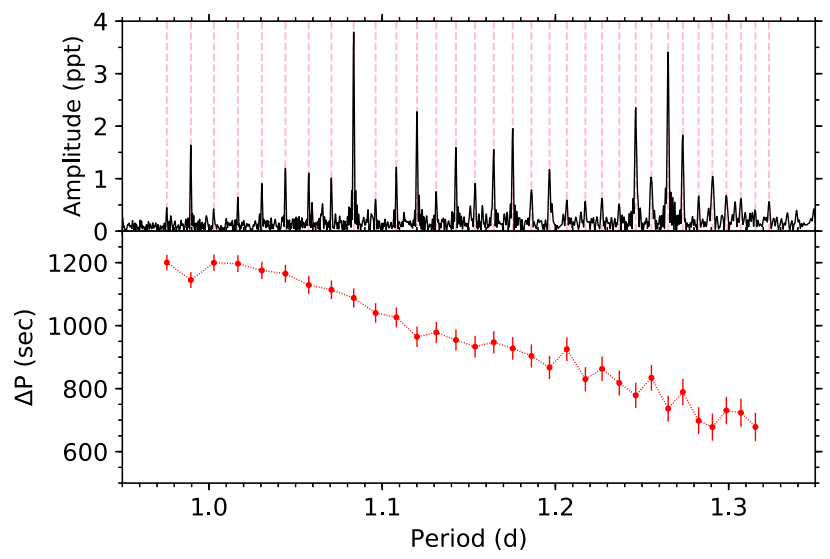

FIG. 11. Top panel: observed amplitude spectrum (black) in terms of the period for the $\gamma$ Dor star KIC 11721304 from its light curve observed with the Kepler satellite. The mode periods with dominant amplitude are indicated with red dashed vertical lines as a guide for the eye. Bottom panel: the period-spacing pattern deduced from the dipole sectoral prograde modes of consecutive radial order $n$ indicated in the top panel. Adapted from Van Reeth et al., 2015.

radial order could be deduced from a 137-d-long light curve by Degroote et al. (2010a). This star revealed periodic deviations from a uniform spacing, which is in line with the theoretical predictions by Miglio et al. (2008). This detection allowed researchers to assess the level of $D_{\mathrm{ov}}$ and to derive an upper limit for $D_{\text {mix }}(r)$ in the radiative envelope. These results were confirmed by independent asteroseismic modeling by $\mathrm{Wu}$ and $\mathrm{Li}(2019)$.

Given the immense asteroseismic potential of g-mode period-spacing patterns, the CoRoT discovery opened the floodgates in the hunt for such patterns in SPB and $\gamma$ Dor stars, once the 4-yr light curves of the Kepler spacecraft became available. Meanwhile $\Pi_{0}$ has been measured for hundreds of stars, one of which is shown in Fig. 11. It can be seen that a clear pattern emerges from the data but that $\Delta P$ is not constant as predicted by Eq. (41). Rather, it decreases for increasing mode period and reveals substructures. Such "tilted" $\Delta P$ patterns turn out to be common in Kepler data of SPB stars, as found by Pápics et al. $(2015,2017)$ and Szewczuk and Daszyńska-Daszkiewicz (2018) and of $\gamma$ Dor stars as revealed by Bedding et al. (2015), Keen et al. (2015), Van Reeth et al. (2015), Ouazzani et al. (2017), Li, Bedding et al. (2019), Li, Van Reeth et al. (2019), and Li et al. (2020). The slope in these observed g-mode $\Delta P$ patterns is caused by the rotation frequency of the star as deduced by Van Reeth, Tkachenko, and Aerts (2016), Ouazzani et al. (2017), Christophe et al. (2018), and Li et al. (2020). This rotation frequency turns out to be of a similar order as the $g$-mode frequencies and puts these modes into the gravitoinertial regime (Aerts, Van Reeth, and Tkachenko, 2017). This implies the need to include the Coriolis force into the theory at the level of the pulsation equations for a proper asteroseismic interpretation. We do so in Secs. II.B.3 and II.B.4, following the recent review on angular momentum transport by Aerts, Mathis, and Rogers (2019), which includes more details and illustrations. 


\section{Rotational splitting in a perturbative approach}

Thus far we have simplified the equations to compute the stellar oscillations by ignoring the stellar rotation. Rotation affects the observed oscillation frequencies in several ways. We choose a reference frame with the polar axis along the rotation axis of the star and corotating with the star under the assumption of a constant rotation frequency $\Omega$. This leads to a purely geometric shift of

$$
\omega_{n l m}=\omega_{n l}+m \Omega
$$

for a mode with frequency $\omega_{n l}$ in the nonrotating case. Further, both the Coriolis and centrifugal forces come into play in the stellar structure equations; see Eq. (30). The Coriolis force lifts the degeneracy of the modes in the star with respect to the azimuthal order $m$. Each mode frequency $\omega_{n l}$ of the eigenvector $\xi_{n l}=\left(\xi_{r, n l}, \xi_{h, n l}\right)$ for the nonrotating case gets split into $2 l+1$ frequency multiplet components due to the influence of the Coriolis force. Hence, each mode degree $l$ can occur with $2 l+1$ different values for $m$, namely, $-l,-l+1, \ldots,-1,0$, $1, \ldots, l-1, l$. Moreover, each of these multiplet components gets shifted over $m \Omega$ as in Eq. (43) in the inertial coordinate system of the observer.

We now consider the case where the so-called spin parameter $s=2 \Omega / \omega \ll 1$ for all involved mode frequencies, with $\omega$ an abbreviated global notation for the oscillation frequencies in the corotating frame. This allows us to treat the Coriolis force as a small perturbation in the pulsation equations. This condition is usually met for $\mathrm{p}$ modes in low-mass stars with convective envelopes, for $\mathrm{p}$ and mixed modes in red giants, and for g modes in subdwarfs and white dwarfs, all of which are slow rotators. This simplification is not justified for the $\mathrm{g}$ modes observed in the majority of intermediate- and high-mass dwarfs, as these modes occur in the gravitoinertial regime and require the Coriolis force to be treated nonperturbatively (Aerts, Van Reeth, and Tkachenko, 2017). We return to the case of gravitoinertial modes later but first treat the easier case of a perturbative treatment of the Coriolis force.

Following the same arguments as for the shellular rotation, we simplify the problem to be solved by assuming that the rotation profile depends only on the radial coordinate $\Omega(r)$. In this case, multiplet components in the inertial coordinate system are, up to first order in $\Omega(r)$, given by [see Unno et al. (1989), Chap. 6 and Sec. 3.8, and Aerts, ChristensenDalsgaard, and Kurtz (2010) for the derivations]

$$
\omega_{n l m}=\omega_{n l}+m\left(1-C_{n l}\right) \int_{0}^{R} K_{n l}(r) \Omega(r) \mathrm{d} r,
$$

where

$$
K_{n l}(r)=\frac{\left\{\xi_{r}{ }^{2}+[l(l+1)] \xi_{h}^{2}-2 \xi_{r} \xi_{h}-\xi_{h}^{2}\right\} r^{2} \rho}{\int_{0}^{R}\left\{\xi_{r}{ }^{2}+[l(l+1)] \xi_{h}^{2}-2 \xi_{r} \xi_{h}-\xi_{h}^{2}\right\} r^{2} \rho \mathrm{d} r}
$$

is the rotational kernel and

$$
C_{n l}=\frac{\int_{0}^{R}\left(2 \xi_{r} \xi_{h}+\xi_{h}^{2}\right) r^{2} \rho \mathrm{d} r}{\int_{0}^{R}\left\{\xi_{r}^{2}+[l(l+1)] \xi_{h}^{2}\right\} r^{2} \rho \mathrm{d} r}
$$

is the Ledoux constant (Ledoux, 1951). Rotational kernels for dipole and quadrupole modes of four radial orders are plotted in the right panels of Fig. 9 for a $1.7 M_{\odot}$ star halfway through its core-hydrogen-burning stage. It can be seen that the highorder $g$ modes have far better probing potential for the core regions of the star than the low-order modes. It is then understood from the profile shape of $K_{n l}(r)$, which acts as a weighting function to the rotation profile, why it is far easier to estimate the near-core values of $\Omega(r)$ than the envelope values for $\mathrm{g}$ modes in stars with a convective core and a radiative envelope once rotational splitting has been detected from data. Finally, we see from Eqs. (45) and (46) that they depend on the equilibrium model via its density profile $\rho(r)$ and its influence on the eigenfunctions.

In the limit of high-order or high-degree $p$ modes, one can show that $C_{n l} \simeq 0$ and $\xi_{r}>\xi_{h}$; see Aerts, ChristensenDalsgaard, and Kurtz (2010), Sec. 3.8. On the other hand, for high-order high-degree $g$ modes, one has $\xi_{r}<\xi_{h}$ as shown in Fig. 9 and one may neglect the terms with $\xi_{r}$ in Eqs. (45) and (46). In this way, the simplification

$$
C_{n l} \simeq \frac{1}{l(l+1)}
$$

emerges. For uniform rotation, this implies that the measured rotational splitting provides a good measure of the average of $\Omega(r)$, weighted with the squared eigenfunction. A further simplification is useful, as Van Reeth et al. (2018) showed that intermediate-mass stars with a convective core are quasiuniform rotators. In the case of constant $\Omega$ one has

$$
\omega_{n l m}=\omega_{n l}+m\left(1-C_{n l}\right) \Omega,
$$

and $C_{n l}$ fully determines the shifts of the frequencies due to the Coriolis force; i.e., those shifts do not depend on the rotational kernels. This means that the adjacent frequencies in a high-order p-mode multiplet belonging to $m=-l, \ldots,+l$ give a direct measure of the average rotation frequency in the stellar envelope without depending on the equilibrium model (because $C_{n l} \simeq 0$ ). In the case of high-order dipole $\mathrm{g}$ modes, $\Omega$ is found to be twice the splitting value in a triplet since $C_{n l} \simeq 1 / 2$.

More complicated perturbative approaches treating the Coriolis force up to second and third order at the level of the pulsation computations, while still relying on 1D equilibrium models, have been developed. We refrain from including the results here for conciseness and refer the interested reader to Saio (1981), Dziembowski and Goode (1992), Lee and Baraffe (1995), Soufi, Goupil, and Dziembowski (1998), DaszyńskaDaszkiewicz et al. (2002), and Suárez, Goupil, and Morel (2006), and Suárez et al. (2010). Few of those theories have been applied to modern space photometric data because the stars for which they are most appropriate are rapidly rotating p-mode pulsators, such as $\delta$ Sct stars and $\beta$ Cep stars; cf. Fig. 1. In the case of the $\delta$ Sct stars, lack of mode identification prevents applications, although Bedding et al. (2020) managed to overcome this hurdle for a limited sample of such stars. The few observed $\beta$ Cep stars with precise space photometry either lack mode identification (Burssens et al., 2019) or rotate slow enough to stick to the first-order perturbative approach. Suárez 
et al. (2010) made a careful analysis of second-order effects in $\Omega$ for stochastic p modes and found those to become important for equatorial rotation velocities above some $15 \mathrm{~km} \mathrm{~s}^{-1}$. This is also the limiting value for the treatment of $\mathrm{g}$ modes in intermediate-mass stars derived by Schmid and Aerts (2016). For rotation speeds above this value, a perturbative analysis should be abandoned as illustrated from their asteroseismic modeling of the high-order $g$ modes in the two F-type p- and g-mode hybrid pulsators in the eccentric binary KIC 10080943. For faster rotation, the g modes enter into the gravitoinertial regime, where one can no longer treat the Coriolis force perturbatively $(s>1)$. As outlined by Aerts, Van Reeth, and Tkachenko (2017) and shown in Fig. 6, this is the case for the observed $g$ modes in almost all intermediateand high-mass stars. We thus conclude that the treatment of $g$ modes in stars with a convective core requires a nonperturbative treatment of the Coriolis force.

\section{Gravitoinertial modes in the traditional approximation}

A major achievement resulting from the 4-yr light curves assembled with the Kepler satellite is the discovery of $g$ modes with period-spacing patterns such as the one illustrated in Fig. 11 in hundreds of stars covering spectral types early-F to early-B along the main sequence; cf. Fig. 1. Except for the few (less than 10\%) stars for which a surface magnetic field was detected in this range of spectral type (Wade et al., 2016), such stars are in general moderate to fast rotators. They are indeed not subject to braking due to the lack of a magnetic field, which does occur in low-mass stars with an appreciable convective envelope. The high-order g modes in these stars of intermediate mass have periods similar to their rotation period so the oscillations are gravitoinertial modes; cf. Fig. 5 given by Aerts, Mathis, and Rogers (2019). Van Reeth, Tkachenko, and Aerts (2016) and Aerts, Van Reeth, and Tkachenko (2017) computed the spin parameters for more than $1650 \mathrm{~g}$ modes in $37 \gamma$ Dor stars and found the majority to have subinertial values, defined as the regime for which $s>1$. In practice, the spin parameter covered values $s=2 \Omega / \omega_{n l m}^{\mathrm{co}} \in[1,15]$, where $\omega_{n l m}^{\mathrm{co}}$ is the mode frequency in the corotating frame.

Taking full account of the Coriolis force in the equation of momentum conservation, even in the adiabatic and Cowling approximations while ignoring the centrifugal force, does not lead to separability of the pulsation equations in terms of the coordinates $(r, \theta, \phi)$. This is why Lee and Saio (1987a, 1987b, 1989) considered the so-called traditional approximation of rotation (TAR) in their theoretical studies of low-frequency $g$ modes. In the TAR, one ignores the horizontal component of the rotation vector such that the equations can be separated in each of the coordinates. This approximation leads us to the Laplace tidal equations (Laplace, 1799), which are commonly used in geophysics (Eckart, 1960). The TAR is a particularly good approximation for the $\mathrm{g}$ modes in intermediate- and high-mass main-sequence stars [as well as in neutron stars; cf. Bildsten, Ushomirsky, and Cutler (1996)], given that their Lagrangian displacement vector is dominantly horizontal; cf. Fig. 9. For derivations of the pulsation equations in the TAR and their asymptotic analysis in a modern numerical context, see Lee and Saio (1997), Townsend (2003a, 2003b), and Mathis (2013). Here we provide the outcome in concise notation that allows for easy comparison with Eq. (41). For uniform rotation, the TAR leads to the following g-mode period-spacing pattern in the corotating frame of reference:

$$
\Delta P_{l, m, s}^{\mathrm{co}}=\frac{\Pi_{0}}{\sqrt{\lambda_{l m s}}},
$$

with $\lambda_{l m s}$ the eigenvalue of the Laplace tidal equation for the $g$ mode with quantum numbers $(l, m)$ in a star with spin parameter $s$. In the limit of $s \rightarrow 0, \lambda \rightarrow l(l+1)$ is recovered. Numerical computation of the eigenvalues $\lambda_{l m s}$ for a chosen 1D equilibrium model of the star then allows for the identification of $(l, m)$, as well as estimation of the spin parameter along with $\Omega$ from an observed period-spacing pattern as in Fig. 11. This opportunity was developed theoretically by Bouabid et al. (2013) and was put into practice for the past five years after careful frequency analysis based on the 4-yr light curves assembled with the Kepler spacecraft. We highlight some of the recent achievements on asteroseismic derivations of $\Omega(r)$ along with opportunities to estimate $D_{\text {mix }}(r)$ from the periodspacing diagnostics in Sec. IV.

Mathis (2009) generalized the TAR to take into account differential rotation with a profile $\Omega(r, \theta)$, while Mathis and Prat (2019) included the centrifugal force for slightly deformed stars in the case of close-to-uniform rotation, deriving an analytical expression for the period-spacing patterns in the Cowling and other justified approximations. In addition, Prat et al. (2017) derived an asymptotic period spacing for axisymmetric gravitoinertial waves taking into account all the components of the rotation vector; i.e., they went beyond the treatment of the TAR. This work was further generalized by Prat et al. (2018) into an asymptotic theory for gravitoinertial waves for a differential rotation profile $\Omega(r, \theta)$. Finally, Prat et al. (2019) derived a period-spacing expression in the presence of uniform rotation on top of an axisymmetric fossil magnetic field with poloidal and toroidal components. None of these recent new theoretical developments have yet been applied to measured g-mode frequencies. This obviously constitutes several future paths for improved asteroseismic modeling compared to the current state of the art. The Kepler data of gravitoinertial pulsators are currently under study with this purpose.

As a noteworthy side step, we point out that only one intermediate-mass g-mode pulsator with a detected surface magnetic field has been the subject of magnetogravito asteroseismology thus far (Buysschaert et al., 2018). This led to the conclusion that the frequency shifts for g modes due to the Lorentz force are far smaller than those due to the Coriolis force for meaningful values of the interior magnetic field strength (Prat et al., 2019). This is quite different from the case of high-frequency magnetoacoustic modes, which occur on the other side of the frequency spectrum in terms of the validity (or lack thereof) of a perturbative approach to treat the Coriolis and Lorentz forces; cf. Fig. 5 given by Aerts, Mathis, and Rogers (2019). Inspired by the solar oscillations, Gough and Thompson (1990) derived expressions for the pertubation to the eigenfunctions caused by rotation and a magnetic field in the stellar interior. Their asymptotic analysis and numerical results for high-order solar acoustic modes for various magnetic field configurations, including a localized 
magnetic field at the base of the convection zone, provide estimates for the frequency splitting when the magnetic field and rotation vary smoothly. This work is a convenient guide to compare with observations.

Aside from Sun-like stars, the best known magnetic pulsators are the roAp stars, which were discovered in 1978 by Kurtz [see Kurtz (1990) for his review on these stars] and later studied in great detail; see Saio (2014) for a more recent review. These core-hydrogen-burning stars oscillate in high- $n$ low- $l \mathrm{p}$ modes according to an axis that may be misaligned with respect to both the magnetic and rotation axes, although it is usually close to the magnetic axis. Their magnetic field strengths are up to a few thousand gauss, while they are slow rotators and this implies that the Lorentz force is more important than the Coriolis force. This is thus a case where the symmetry axis for the oscillations is inclined with respect to the rotation axis. Although their magnetoacoustic modes have sufficiently high amplitudes and periods of only a few minutes, making them easily accessible from ground-based asteroseismology, their recent studies have benefited greatly from modern space photometry. The oblique pulsator model of roAp stars has constantly been in need of improvement as more data become available, as shown by Shibahashi and Takata (1993) and Bigot and Dziembowski (2002). This model was again challenged and refined thanks to the high-frequency precision obtained from space asteroseismology, which revealed that some roAp stars seem to have multiple pulsation axes (Kurtz et al., 2011) and others oscillate in distorted pulsation modes (Holdsworth et al., 2016). Recent TESS data have been used to find the shortest period roAp star, with a pulsation period of only $4.7 \mathrm{~min}$ (Cunha et al., 2019). A growing number of roAp stars have been found to pulsate above their acoustic cutoff frequency, which presents another challenge to current pulsation theory.

\section{Rossby modes}

We now return to the maximally simplified version of the stellar pulsation equations deduced from perturbing Eq. (19) in the absence of rotation and magnetism. This approach allowed us to introduce the time-dependent spheroidal modes of oscillation known as $\mathrm{p}$ and $\mathrm{g}$ modes. However, if we keep the Coriolis force in Eq. (19) and perturb that version of the equation, then two families of eigenvalue problems actually result, each with nonzero eigenvalues. The first family is the one we have been discussing, leading to spheroidal normal modes of a star. We now pick up the second family of eigensolutions, termed toroidal normal modes. In particular, we consider the Rossby modes, also termed and abbreviated as "r modes" by Papaloizou and Pringle (1978). This is a family of toroidal normal modes that become time dependent (and hence nonzero) only in a rotating star. The dominant restoring force of these modes is the Coriolis force. This is why they cannot be deduced from Eq. (19) unless a nonzero rotation vector is considered.

Toroidal modes comply with $\operatorname{div} \xi=0$ and $\xi_{r}=0$. Therefore, just as with the gravitoinertial modes discussed in Sec. II.B.4, the eigenvalues of Rossby modes can be deduced with excellent precision by adopting the TAR and solving the Laplace tidal equations. For the eigenfrequencies of the spheroidal $\mathrm{g}$ modes we had the limiting case of $\lambda \rightarrow$ $l(l+1)$ as $s \rightarrow 0$. For the Rossby modes, one obtains $\lambda \rightarrow 0$ as $s \rightarrow[l(l+1)] / m$ (Papaloizou and Pringle, 1978). For this reason, Lee and Saio (1997) adopted an ordering of the eigenvalues by introducing a labeling scheme that allows one to treat the cases of gravitoinertial $\mathrm{g}$ modes and pure inertial modes with one set of indices $(k, m)$, with $k=l-|m| \geq 0$ for gravitoinertial modes and $k<0$ for purely inertial modes; see Townsend (2003b) for the various types of low-frequency modes in rotating pulsators. Rossby modes have frequencies below the rotation frequency in the corotating frame and are therefore always retrograde modes in the inertial frame of the observer (Saio, 1982). The occurrence of the temperature variations at the stellar surface due to Rossby modes and for various values of the spin parameter is shown in Fig. 2 given by Saio, Kurtz et al. (2018) and omitted here for conciseness.

With the labeling scheme introduced by Lee and Saio (1997), the period-spacing pattern of Rossby modes becomes

$$
\Delta P_{k m s}^{\mathrm{co}}=\frac{\Pi_{0}}{\sqrt{\lambda_{k m s}}},
$$

with $\lambda_{k m s}$ again the eigenvalues of the Laplace tidal equation. It was shown by Townsend (2003a) that the eigenvalues for Rossby modes comply with $\lambda_{k m s} \approx m^{2}(2|k|-1)^{-2}$ for $s \gg 1$ and $k \leq-2$. From this, it is found that the period-spacing value of Rossby modes of consecutive radial order as seen by an observer increases with increasing mode period. This is illustrated for the $\gamma$ Dor star KIC 12066947 observed by the Kepler spacecraft in Fig. 12. This star has both prograde gravitoinertial dipole modes with $k=0$ and retrograde Rossby modes with $k=-2$. For its sectoral gravitoinertial g modes, just as for the ones observed for KIC 11721304 shown in Fig. 11, the label is $k=0$ and we recover the treatment of the period-spacing pattern represented by Eq. (49).

Van Reeth, Tkachenko, and Aerts (2016) made the first discovery of Rossby modes in Kepler data. This was achieved for ten $\gamma$ Dor stars, all of which were found to have spin parameters $s \in[14,30]$ (Aerts, Van Reeth, and Tkachenko, 2017). Meanwhile, Rossby modes were found to be common in F-, B-, Be-, and A-type stars, as well as in eccentric binaries, all of which were observed using the Kepler spacecraft and studied by Saio, Kurtz et al. (2018) and Li, Van Reeth et al. (2019). These discoveries offer the opportunity to assess whether the interior rotation is constant or differential from combined g- and r-mode asteroseismology. We also note in passing that retrograde Yanai modes have been discovered in Kepler data of seven $\gamma$ Dor stars thus far by Van Reeth et al. (2018) and Li, Van Reeth et al. (2019). As explained by Townsend (2003a), this family of modes behaves like gravitoinertial modes when they are prograde, while the retrograde Yanai modes behave as Rossby modes but have $k=-1$. We show the results for KIC 6425437, which is one such star revealing a period-spacing pattern of Yanai modes, in Fig. 13 (Van Reeth et al., 2018). Just as with the Rossby modes, the full potential of Yanai-mode frequencies in terms of asteroseismic probing of the properties of the interior physics has yet to be investigated and exploited, given their recent discoveries. 

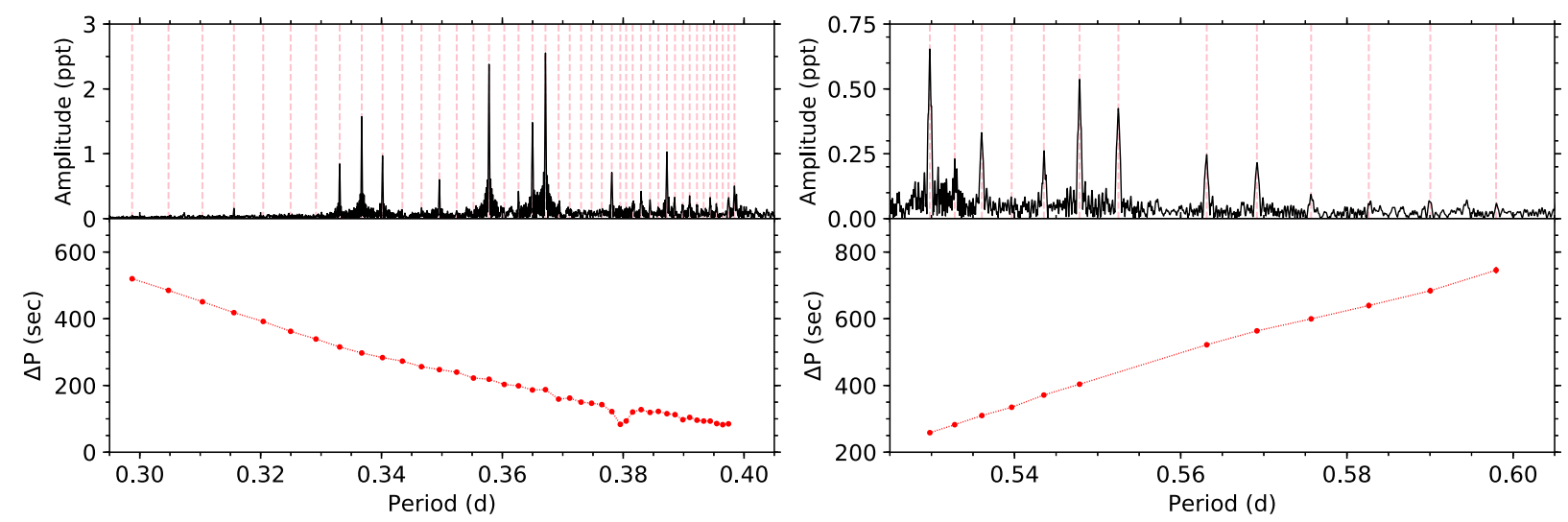

FIG. 12. Same as Fig. 11, but for the $\gamma$ Dor star KIC 12066947 exhibiting both prograde dipole gravitoinertial modes with $(k, m)=$ $(0,+1)$ and retrograde Rossby modes with $(k, m)=(-2,-1)$. In contrast to the case of KIC 11721304 shown in Fig. 11, the errors in the period-spacing pattern are smaller than the symbol size. Adapted from Van Reeth et al., 2015.

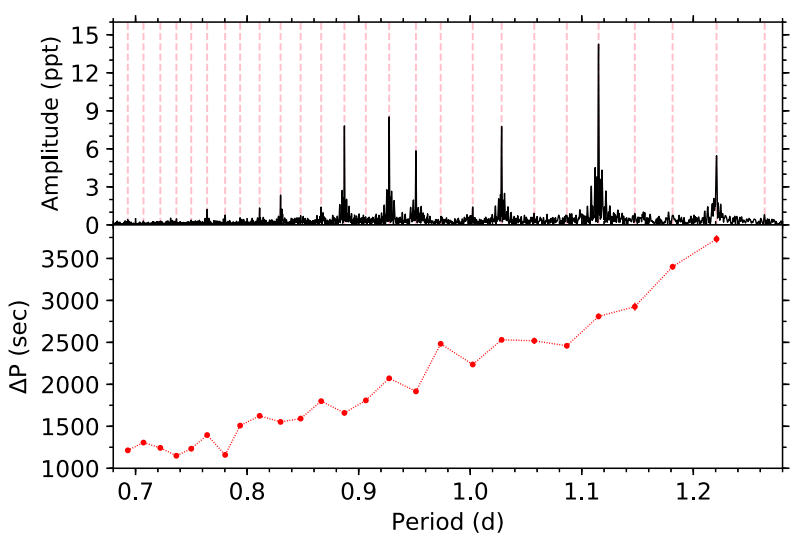

FIG. 13. Same as Fig. 11, but for the $\gamma$ Dor star KIC 6425437 exhibiting retrograde Yanai modes with $(k, m)=(-1,-1)$. In contrast to the case of KIC 11721304 shown in Fig. 11, the errors in the period-spacing pattern are smaller than the symbol size. Adapted from Van Reeth et al., 2015.

\section{PRINCIPLES OF ASTEROSEISMIC MODELING}

The theory of nonradial oscillations outlined in Sec. II rests on the assumption of linearity. Although the amplitudes of stochastically excited modes of the Sun can now be predicted from the damping rate and the stochastic energy from 3D convection simulations (Zhou, Asplund, and Collet, 2019), this is not the case for the amplitudes of the modes excited by other mechanisms. Thus, most interpretations in asteroseismology rely on the properties of the detected mode frequencies and not on their amplitudes. Asteroseismic modeling is therefore usually done in a linear adiabatic framework. After having derived the frequencies of the modes $\omega$ and their uncertainties $\sigma_{\omega}$ from the data, the interpretations in terms of the theory of nonradial oscillations computed from perturbing stellar equilibrium models (cf. Sec. II) can be done only if the modes' identifications have been achieved. This means that we must be able to label the radial order, the degree, and the azimuthal order $(n, l, m)$ for each of the modes corresponding to the measured oscillation frequencies $\omega_{n l m}^{\mathrm{obs}}$.
Mode identification is usually based on patterns recognized from the list of adiabatic frequencies $\omega_{n l m}^{\text {theo }}$, predicted from the perturbation of 1D equilibrium models as outlined in Sec. II. Easily interpretable patterns concern those due to rotational splitting or corresponding to the predictions from the asymptotic theory, as outlined in Sec. II. Comparison between predicted and detected patterns such as those shown in Figs. 10-12 can then be fed with the "wisdom" of the applicant to identify $(n, l, m)$. This wording already indicates why asteroseismologists tend to be "Bayesian minded" when identifying modes and performing asteroseismic modeling; see Bazot, Bourguignon, and Christensen-Dalsgaard (2012), Gruberbauer, Guenther, and Kallinger (2012), Appourchaux (2014), and Aerts et al. (2018b) for thorough discussions. Nevertheless, MLE and model selection with so-called noninformative (flat) priors is often enlightening and sometimes necessary to avoid too much prejudice in the prior, particularly on the appropriateness of the equilibrium models used to compute the mode predictions.

Despite our inability to predict reliably which of the eigenmodes should get excited to observable amplitudes, the mode excitation mechanisms are understood in general terms for the classes in Fig. 1. Therefore, even though the nonadiabatic treatment of the oscillations is not sufficiently established to derive perfect mode excitation and amplitude predictions, it is still instructive to consider the regimes of mode excitation before tackling the task of mode identification and asteroseismic modeling.

\section{A. Excitation mechanisms}

\section{Heat mechanisms and stochastic driving}

Thus far we have ignored the perturbation of the entropy in Eq. (21), which greatly simplifies the theory of nonradial oscillations. However, to get an understanding of mode excitation, nonadiabatic theory needs to be considered. This is extensively discussed in Chaps. IV and V given by Unno et al. (1989) and also in Aerts, Christensen-Dalsgaard, and Kurtz (2010), Sec. 3.7, which addressed the general problem and the development of the so-called quasiadiabatic approximation. The prediction for a mode to get excited relies on the computation of its growth rate. This quantity is positive for 
modes that get excited (or modes that are unstable, as is often used as terminology), while it is negative for modes that are overdamped. Derivation of the growth rate of a mode requires the computation of the imaginary part of its eigenfrequency; see Eq. (3.282) given by Aerts, Christensen-Dalsgaard, and Kurtz (2010). When considering the theoretical expression, one finds that excitation occurs whenever the compression of the gas and its heating happen in phase with each other. This is completely in line with the operation of a thermodynamical heat engine.

The perturbations to both the flux (radiative + convective, $\left.\boldsymbol{F}=\boldsymbol{F}_{\text {rad }}+\boldsymbol{F}_{\text {conv }}\right)$ and the energy generation stemming from Eq. (21) go into the overall expression for the heat. For each of these three contributions, one adopts a specific terminology. When the perturbation of the energy generation is dominant in the heat term that sets the imaginary part of the mode frequency [cf. Eq. (21)] the $\varepsilon$ mechanism may operate. This can obviously happen only in the deep stellar interior. In the case in which the radiative flux delivers most of the heat, it is often due to the increased opacity that acts as the heat engine in the thin partial ionization layers in the envelope of the star. This driving of oscillations is therefore often called the $\kappa$ mechanism, giving rise to self-excited modes with infinite lifetimes. Theoretical predictions of nonradial mode excitation via the $\kappa$ mechanism in intermediate-mass stars along the main sequence are generally good. Pamyatnykh (1999), Bouabid et al. (2013), and Szewczuk and Daszyńska-Daszkiewicz (2017) conducted extensive studies. Yet they are not perfect: we observe more modes than predicted for OB-type pulsators, particularly in the $g$-mode regime. Bringing theory and observations into agreement requires either higher-than-standard opacities in the partial ionization zones of ironlike species situated in the layers with temperatures $\sim 2 \times 10^{5} \mathrm{~K}$, as shown by Moravveji (2016) and Daszyńska-Daszkiewicz et al. (2017), or higher metal abundances in the local region of the excitation (for instance, as a consequence of atomic diffusion). Similarly, iron and nickel opacity enhancements are needed to explain the $\mathrm{g}$ modes observed in cool pulsating subdwarf B stars, as emphasized by Fontaine et al. (2003), Jeffery and Saio (2006), and Bloemen et al. (2014). Their hotter counterparts were predicted theoretically by Charpinet et al. (1997) in terms of $\mathrm{p}$ modes excited by the $\kappa$ mechanism at about the same time as, but independently of, their observational discovery by Kilkenny et al. (1997). Opacity bumps due to carbon and oxygen in layers of $\sim 10^{6} \mathrm{~K}$ result in heat-driven mode excitation of helium-rich subdwarfs (Saio and Jeffery, 2019) and GW Vir variables (also known as DO white dwarfs). Córsico et al. (2019) provided a recent summary of pulsating white dwarfs, and Montgomery et al. (2020) inferred nonstatic convection zones in white dwarfs from observational limits on their mode coherence.

The perturbation to the convective flux and its contribution to the heat presents a much larger challenge than the case of radiative flux, because it is coupled to the properties of the turbulent pressure. For the deep stellar interior, one may assume that this is time independent and well described by mlt. However, for convective outer envelopes, the perturbations to the convective flux and the turbulent pressure render the modes stable such that the heat-engine mechanism does not drive oscillations. Instead, excitation occurs through stochastic forcing, where the energy in the acoustic noise in the outer convection zone triggers some of the global eigenmodes. This stochastic forcing happens in stars with an outer convective envelope, leading to the excitation of damped and continuously reexcited oscillation modes. In this case, predictions of the excitation and properties of the modes are challenging due to the limited knowledge of the timedependent properties of $\boldsymbol{F}_{\text {conv }}$ in the equilibrium models. This propogates into theoretical uncertainty for the perturbation of the time-variable convective envelope of a pulsating star.

In the limit of extremely long convective timescales relative to the periods of the oscillations, the convective flux does not react to the pulsations and convective flux blocking becomes an efficient excitation mechanism. This excites g modes in the thin convective envelopes of the $\gamma$ Dor stars, as shown by Guzik et al. (2000) and further elaborated upon by Dupret et al. (2005). Gravitoinertial modes in the radiative envelope of such stars may couple resonantly to inertial modes in the convective core of rapid rotators (Ouazzani et al., 2020). On the other hand, Lee and Saio (2020) found mode excitation due to resonant coupling between convective $\mathrm{g}^{-}$modes active in the core of rapidly rotating $2 M_{\odot}$ stellar models and $\mathrm{g}$ modes in the radiative envelope, for frequencies $|m| \Omega_{\text {core }}$. For the intermediate-mass $\delta$ Sct and $\gamma$ Dor stars, the time dependence in the pulsationconvection interaction is known as the problem of the red edge of the instability strip; cf. Fig. 1. The convective timescales in the thin outer convection zones of DA and DB white dwarfs are much shorter than those of their g-mode pulsation periods, leading to mode excitation (Goldreich and $\mathrm{Wu}, 1999)$.

The general case where the convective and mode timescales are similar is much more challenging to treat in terms of stochastic mode excitation by the turbulent pressure perturbation. This was developed by Houdek et al. (1999) and Dziembowski et al. (2001). Major improvements in excitation theory were achieved for low-mass stars across stellar evolution by Belkacem et al. (2008, 2011, 2012), Dupret et al. (2009), and Grosjean et al. (2014); Houdek and Dupret (2015) provided a good summary. Despite this progress, considerable uncertainty in the predictions for mode excitation and damping properties, as well as for the amplitudes, remain due to uncertainties in the equilibrium structure of the superadiabatic outer envelope and its coupling to the atmosphere. Convection and nonadiabaticity also affect the oscillation frequencies, and despite major efforts and progress on this front for the Sun (Houdek et al., 2017), current theoretically predicted frequency values are not yet at a level such that they can be fitted to the observed frequencies. This is known as the problem of the "surface effects" in asteroseismology of stars with solarlike oscillations.

A rough global summary of the observed mode periods and amplitudes in pulsators excited by heat mechanisms and stochastic driving for the classes indicated in Fig. 1 is provided in Table A.1 given by Aerts, Christensen-Dalsgaard, and Kurtz (2010). The periods range from minutes to months. While this is already a broad range, at least three more additional cases of mode excitation are in order.

\section{Nonlinear resonant mode excitation}

Many of the CoRoT and Kepler light curves reveal nonlinear effects. Combination frequencies are omnipresent in the 
oscillation spectra of $\kappa$-driven pulsators along the main sequence, as discussed by Degroote et al. (2009), Pápics (2012), Kurtz et al. (2015), and Bowman et al. (2016). Combination frequencies got lost in ground-based data, as they often have amplitudes below ppt. They may be due to nonlinearities in the light curves due to deviations from sinusoidal variations because the modes have amplitudes beyond the linear regime. However, given the density of gmode eigenfrequency spectra, combination frequencies may also occur at actual eigenmode frequencies of the star that get excited by nonlinear resonant mode coupling. A distinction between these two cases is not evident when dealing with hundreds of frequencies deduced from a long-duration light curve.

Excitation of nonradial "daughter" modes via nonlinear "parent" mode coupling is expected for particular low-order combination frequencies from theoretical considerations based on the method of amplitude equations, as developed by Buchler and Goupil (1984), Goupil and Buchler (1994), Van Hoolst (1994), and Buchler, Goupil, and Hansen (1997). This mode excitation may give rise to time-variable mode amplitudes, as commonly observed in space photometry of the higher-amplitude nonradial pulsators. Mainly due to a lack of proper data, theoretical predictions on nonlinear mode excitation remained largely unexploited prior to space asteroseismology, with the notable exception of $\mathrm{g}$ modes in white dwarfs. For those, the phenomenon of nonlinear mode coupling was already accessible from ground-based data, thanks to their short pulsation periods. This allowed one to assess the depth of the outer convection zones following $\mathrm{Wu}$ (2001) and Montgomery (2005). Meanwhile, nonlinear mode interactions were detected in the short-cadence Kepler data in the cool pulsating DA white dwarfs KIC 4552982 (Bell et al., 2015) and PA $1149+057$ (Hermes et al., 2015). Both of these DA pulsators revealed large-amplitude regular outbursts with timescales of days, which is much longer than the individual pulsation-mode periods. This nonlinear behavior in pulsating white dwarfs remained unknown prior to space asteroseismology, even though large flux variations up to $\sim 20 \%$ recur. Nonlinear asteroseismology was also performed for the DB white-dwarf star KIC 8626021 (Zong, Charpinet, and Vauclair, 2016) and for the pulsating subdwarf B star KIC 10139564 (Zong et al., 2016), where rotation was found to be a key actor in the detected resonances of the latter.

Nonlinear mode coupling behaves in diverse ways, as predicted by theory, and it is a phenomenon occurring across the entire HRD. Weinberg and Arras (2019) invoked cascades of daughter modes resulting from nonlinear mixed-mode parents as an explanation for the suppression of mixed-mode amplitudes observed in about a quarter of the pulsating red giants. This study was triggered by the suggestion made by Mosser, Belkacem et al. (2017) that not all suppressed dipole modes found in red-giant pulsators with this phenomenon can be explained by the magnetic greenhouse effect originally proposed by Stello et al. (2016). Indeed, some of the stochastic dipole modes with depressed amplitudes are mixed modes with a g-mode character in the stellar interior rather than $\mathrm{p}$ modes, as assumed in theoretical developments by Fuller et al. (2015) and Cantiello, Fuller, and Bildsten (2016). Although progress in interpretations based on a strong internal magnetic field was achieved by Loi (2020), Loi and Papaloizou (2020), and Bugnet et al. (2021), the interpretation by Weinberg and Arras (2019) does not require core magnetism. This nonlinear theory is an alternative and complementary explanation, which is in line with Kepler data of intermediate-mass dwarfs revealing $\mathrm{g}$ modes (SPB and $\gamma$ Dor stars in Fig. 1). Their period-spacing patterns would be affected by a strong magnetic field, following the theory by Prat et al. $(2019,2020)$ and accompanying predictions by Van Beeck et al. (2020). To date there has been no observational evidence of internal magnetic fields from observed g-mode period spacings of intermediate-mass stars revealed by Van Reeth et al. (2015), Pápics et al. (2017), Li, Bedding et al. (2019), Li, Van Reeth et al. (2019), and Li et al. (2020).

This brings us to nonlinear nonradial asteroseismology for $\kappa$-driven main-sequence stars from Kepler data. This underdeveloped research field within asteroseismology holds great potential, but the theory is still to be refined up to the level of the Kepler data. Unraveling nonlinear effects in the light curves from nonlinear mode coupling via resonant excitation is possible in principle for modes with an infinite lifetime, as the two lead to distinguishable properties. A distinction between the two cases can be made via the phase behavior of the combination frequencies of parent and daughter modes. One expects phase locking to take place whenever low-order combination frequencies occur exactly at another eigenfrequency of the star, such that the latter gets excited by energy exchange between the two or more parent and daughter modes involved in the resonance. Such phase locking was observed in the CoRoT data of the large-amplitude $\beta$ Cep star HD 180642 (Degroote et al., 2009). Energy exchange due to resonantly coupled nonradial g modes was invoked as the cause of outbursts in pulsating Be stars, a phenomenon first observed in a Be star by the CoRoT satellite (Huat et al., 2009), and later for several Be pulsators with the BRITE constellation (Baade et al., 2018). Detailed mode coupling studies are currently being undertaken from Kepler longcadence data of $\mathrm{p}$ - and g-mode pulsators along the main sequence; see Saio, Bedding et al. (2018) for a $\gamma$ Dor pulsator. Given the observed amplitude and frequency modulations in numerous $\delta$ Sct and $\gamma$ Dor stars (Bowman, 2017), the prospects for data-driven nonlinear asteroseismology to be put into practice are excellent. Even if mode excitation by nonlinear resonances is not completely understood, one can take the pragmatic approach of exploiting the detected combination frequencies involved in resonance locking and, once identified, test to see whether adding them to the list of identified pulsation modes used for asteroseismic inferences improves in terms of precision the inferred internal physics. A good target to test this is the Kepler eclipsing binary KIC 3230227 (Guo, 2020).

\section{Convectively driven internal gravity waves}

Traveling damped IGWs can be generated at the interfaces between convective and radiative zones by turbulent convective flux forcing, as studied for the Sun by Rogers and Glatzmaier (2005) and for solar-type stars by Dintrans et al. (2005). The pioneering study by Charbonnel and Talon (2005) showed that inward traveling IGWs in low-mass stars result in 
retrograde waves with the capacity to impose near-rigid rotation on timescales much shorter than the evolutionary timescale. For intermediate- and high-mass stars, IGWs travel outward from the convective core with a similar capacity (Rogers et al., 2013), which explains observed asteroseismic rotation properties (Rogers, 2015).

Although the 3D simulations of solarlike stars by Alvan, Brun, and Mathis (2014) and Alvan et al. (2015) and of intermediate-mass stars by Edelmann et al. (2019) and Horst et al. (2020) showed clear modal structure of internal g modes, it remains unclear if and which of the internal waves become resonant modes. This depends on the profile of the wave's eigenfunction, on its propagation and dissipation properties, on the efficiency of the radiative damping, and on the onset of nonlinearity (Ratnasingam, Edelmann, and Rogers, 2019). A snapshot of the temperature variations accompanying the driving of IGWs and their propagation from the 3D hydrodynamical simulations by Edelmann et al. (2019) is shown in Fig. 14. This gives the reader a grasp of the large-scale fluctuations induced in the stellar interior. The theoretical predictions coupled to the setup of the 3D hydrodynamical simulations is subject of intense debate among various research teams, because it is hard to drive IGWs from convective flows by heating. For this reason, most numerical simulations adopt artificial luminosity boosting in the convective region to get the flows going into the radiative region at velocities compliant with mlt. The dependence of IGW behavior on the level of boosting remains to be studied in detail. The fully compressible 2D simulations by Horst et al. (2020) have lower numerical viscosity and a factor of 1000 lower luminosity boosting than the 3D simulations by Edelmann et al. (2019), yet lead to similar results in terms of IGW properties. Moreover, these simulations lead to

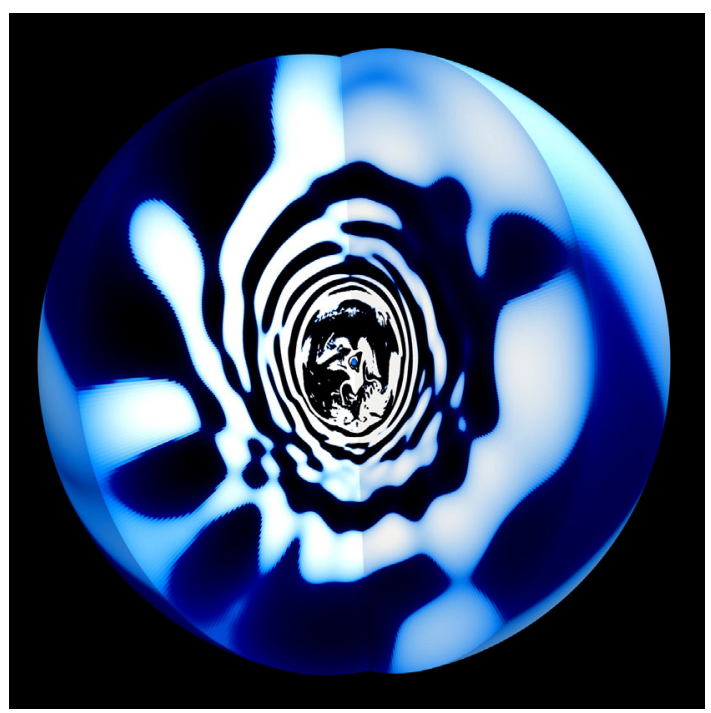

FIG. 14. Snapshot of 3D hydrodynamical simulations representing the temperature fluctuations induced by IGWs excited by stochastic forcing at the transition layer between the convective core and the bottom of the radiative envelope of a $3 M_{\odot}$ ZAMS star. The color coding represents fluctuations up to $10^{5} \mathrm{~K}$ with respect to an equilibrium model. Adapted from Edelmann et al., 2019. appropriate predictions for $\mathrm{p}$ modes and for SLF variability in agreement with space observations of high-mass stars (Bowman et al., 2019a, 2019b, 2020).

The overall spectra of IGWs can be triggered by convective cores, convective envelopes, or thin convection zones due to shell burning or opacity bumps in radiative envelopes. These various cases of IGW generation were extensively discussed by Talon and Charbonnel (2008), Cantiello et al. (2009), Rogers et al. (2013), and Fuller et al. (2014), respectively. Because of an inability to predict which of the waves within the entire generated spectrum of IGWs could get excited as resonant $\mathrm{g}$ modes with observable amplitude, we are still far from pinpointing their $\lambda_{l m s}$ values from SLF detected in space photometry, as shown in the right panels of Fig. 4. Just as with the observed g modes, convectively triggered waves will occur mostly in the gravitoinertial regime for the majority of mainsequence intermediate- and high-mass stars, because these waves have spin parameters $s>1$ for the measured rotation rates of such stars. In view of this, theoretical and numerical studies should consider the driving, propagation, and dissipation of stochastic gravitoinertial waves (GIWs) in rotating stars (Augustson, Mathis, and Astoul, 2020), rather than IGWs in nonrotating stars. Synergies between GIWs predicted from 3D simulations and nonradial nonadiabatic oscillation modes computed in the TAR from 1D stellar equilibrium models are yet to be explored, starting with the observational constraints on the detected frequency regimes, in the spirit of Fig. 1 given by Aerts, Mathis, and Rogers (2019). In that way, one may develop asteroseismology based on the observed spectra of the GIWs. Neiner et al. (2020) offer a step in this direction with their application of GIW asteroseismology to the rapidly rotating pulsating Be star HD 49330 observed by CoRoT.

\section{Tidal excitation of nonradial modes}

The tidal action of a companion in a close binary is yet another way to excite nonradial oscillation modes. This was realized by Cowling (1941) when he introduced "his" Cowling approximation. Tidally excited nonradial oscillations and their effect on stellar evolution have been studied extensively in the literature from a theoretical viewpoint for various types of close binaries; see Zahn (1975), Papaloizou and Savonije (1997), Savonije and Papaloizou (1997), Terquem et al. (1998), Witte and Savonije (1999), Willems (2003), and Fuller and Lai (2011) for studies across the stellar mass range prior to the Kepler mission. Fuller (2017) covered the case of eccentric binaries discovered from space photometry. For this excitation mechanism to work, the properties of the nonradial eigenmodes must be "suitable" compared to the period and the eccentricity of the binary orbit. The component masses and radii must also be in the proper regime to trigger nonradial modes by the tidal forces. The tide-generating potential within an eccentric binary is related to an infinite number of partial dynamic tides with forcing frequencies and it is dominated by spherical harmonics of degree $l=2$. Whenever one of those forcing frequencies gets into resonance with an eigenfrequency of a free oscillation mode of one of the components, the tidal action exerted by the companion may excite this mode. 
Tidally excited oscillations are expected to occur at exact multiples of the orbital frequency. This makes them easy to spot in the Fourier transforms of the light curves, particularly when they occur in eclipsing binaries for which the orbital period is directly accessible from the space photometry. The occurrence of resonances between partial dynamic tides and free oscillation modes is particularly relevant for the excitation of $g$ modes, because of their similarity in period compared to orbital periods of close binaries. Just as for the $\mathrm{g}$ modes in single stars, CoRoT already allowed us to discover tidally excited modes in binaries, but the 4-yr-long time base of the Kepler mission implied the true beginnings of tidal asteroseismology. Numerous cases have meanwhile been discovered and analyzed, the most spectacular one shown in Fig. 15 (another one is discussed in Sec. IV.F and is shown in Fig. 19). The stunning light curve of KIC 8112039, also known as Kepler object of interest number 54 (KOI-54) (Welsh et al., 2011), shown in Fig. 15 was the first object of a new class of high-eccentricity pulsating binaries whose Kepler light curve resembles the signal of a human heartbeat measured in a cardiogram, hence this class was named "heartbeat stars" by Thompson et al. (2012). However, we prefer a naming based on the physical properties, and hence refer to the class as higheccentric binaries. KOI-54 has more than 100 tidally excited $g$ modes, indicated by the red vertical lines in Fig. 15. Those with dominant amplitude occur at 90 and 91 times the orbital frequency and are interpreted in terms of prograde sectoral quadrupole modes excited by dynamical tides in a system where the rotation axis of the primary star is almost aligned with the orbital axis. These two dominant modes are locked in resonance with the orbit. Such an interpretation leads to the other $\mathrm{g}$ modes being near-resonant quadrupole zonal modes, while the two modes whose frequency is not a multiple of the orbital frequency are due to three-mode nonlinear mode coupling (Fuller and Lai, 2012). With the discovery of numerous close binaries revealing multiple oscillation modes, tides are an important excitation mechanism for nonradial modes in binaries, while they also affect free oscillation modes that would occur if the star were single (Guo et al., 2020). The interpretation of tidally excited or tidally affected nonradial modes is a tedious job, because the tidal forces imply deformations of the mode cavities relative to the cavities for single stars; cf. Fig. 8. This bare fact has yet to be exploited in detail.

The opposite situation of having fewer modes than expected also occurs and may be connected to binarity. Evidence for suppression or even absence of solarlike oscillations in lowmass stars with convective envelopes by stellar companions was found (Derekas et al., 2011; Gaulme et al., 2016; Schonhut-Stasik et al., 2020). Systematic large-scale observational studies with spectroscopy, interferometry, or adaptive optics are required to deduce the cause(s) of the absence of expected oscillations in unknown spectroscopic binaries, e.g., to discriminate between tidal changes of the mode cavities versus dilution of oscillation amplitudes due to contaminating flux from visual companions. Evidence for damping of solarlike oscillations or amplitude suppression due to magnetic activity has also piled up for both single and binary lowmass stars (Mathur et al., 2019).

As a general conclusion, one cannot rely on current theory to deliver a complete list of unstable mode frequencies $\omega_{n l m}^{\text {theo }}$ to be compared with the observed ones to identify the mode wave numbers belonging to each detected frequency. We observe more eigenmodes than predicted by the current nonadiabatic
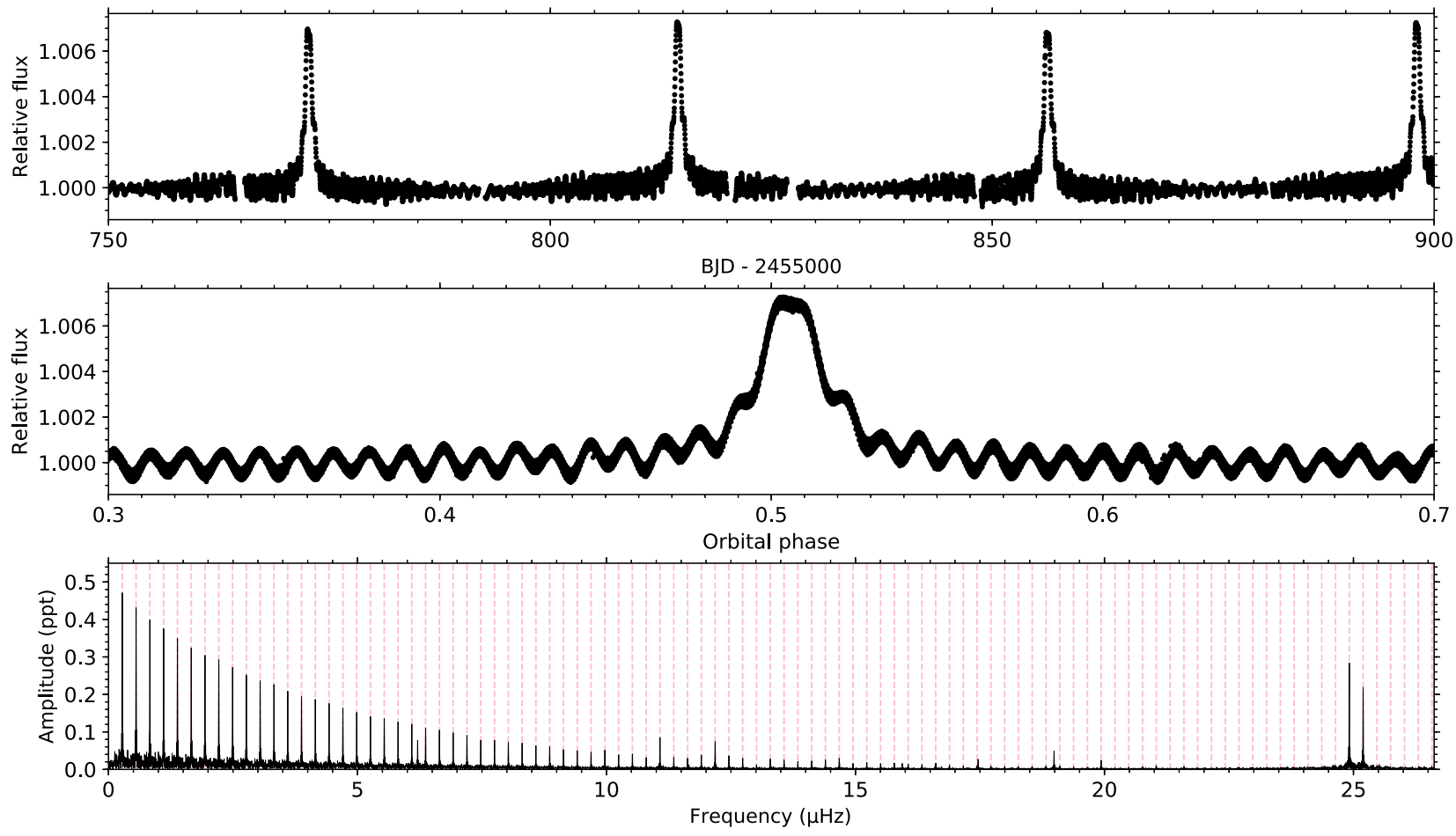

FIG. 15. Excerpt of the Kepler light curve (top panel), phase folded according to the orbital frequency (middle panel), and LS amplitude spectrum (bottom panel) of the eccentric eclipsing binary KIC 8112039. All but two frequencies (not indicated by a thin red vertical line) in the bottom panel are caused by tidal excitation. Adapted from Welsh et al., 2011. 
nonradial oscillation theory, particularly in the g-mode regime. This observational fact points to current limitations in nonadiabatic mode excitation and damping computations, due to missing opacity, to overinterpretation of radiative damping, or to yet other unknown physical phenomena in the outer envelopes of stars. It is therefore premature to rely on nonadiabatic predictions of mode excitation when performing asteroseismic modeling. Instead, the mode excitation predictions should be used as a good but not perfect guideline of the modes to be expected from the current knowledge of input physics in equilibrium models while being aware that modes predicted not to be excited do occur in real stars, and the other way around. Once the best stellar model has been found from adiabatic asteroseismic modeling, one can check its nonadiabatic mode excitation and damping predictions and use those modes that are predicted to be stable yet observed as an excellent guide to improve the input physics of the models and the excitation theory.

\section{B. Mode identification}

Inferences on stellar interiors from asteroseismology provide tighter constraints the more oscillation modes are involved in the modeling. For this reason, asteroseismic modeling takes a pragmatic data-driven approach: we thankfully use all detected frequencies offered by the stars to work with as long as we can label their wave numbers $(l, m, n)$ or $(k, m, n)$ from adiabatic eigenfrequency predictions. When uncertainty in the labeling occurs, the frequency can still be used but the best equilibrium model selection should be done in a Bayesian way, encapsulating the uncertain mode identification in the prior(s). Thanks to space photometry, identification of $(l, m)$ can often be achieved from pattern recognition, notably when rotational multiplets as in Eq. (44) are detected. We now highlight a few of the current methods to deduce the mode identification. These depend on the kind of pulsator and type of mode(s).

It is noteworthy that asteroseismic modeling to estimate stellar properties other than rotation is usually done from axisymmetric $(m=0)$ modes. These tend to be available in low-mass stars with stochastically excited modes. Such modes are often not detected in the case for $\mathrm{p}$, g, or $\mathrm{r}$ modes in heatdriven pulsators. Moderate to fast rotators tend to reveal mostly prograde or retrograde modes with $m \neq 0$ in the data. For such cases, identification of $m$ is also needed, aside from labeling $n$ and $l$ (or $k$ in the case of Rossby modes).

\section{Mode identification from échelle diagrams}

For low-mass stars with stochastically-excited modes, as those shown in Fig. 10, one uses so-called échelle diagrams to identify the $l$ and $n$ values of the modes. An échelle diagram is a plot of the detected mode frequencies as a function of the frequencies modulo the large frequency separation as given in Eqs. (38) and (39) and readily accessible from the PD, as illustrated in Fig. 10. In practice, an observed PD spectrum is cut into segments of length $\Delta \nu$, and these segments are subsequently stacked on top of each other to make a 2D $\operatorname{map}$ of $\nu \operatorname{vs} \nu \bmod (\Delta \nu)$. When doing that, modes of the same degree $l$ "line up" along quasivertical ridges. This was found to be a convenient way to represent and identify the solar oscillation frequencies by Grec, Fossat, and Pomerantz (1980), who introduced the terminology of échelle (French for "ladder") diagram. Échelle diagrams are commonly used ever since to identify $l$ and $n$ in low-mass pulsators with stochastically excited modes [see Fig. 2 given by Chaplin and Miglio (2013), for examples from Kepler data], and recently also for $\mathrm{p}$ modes of young $\delta$ Sct stars (Bedding et al., 2020).

A computationally convenient way to identify modes after derivation of the large and small frequency separation in the case of noisy data was developed by Roxburgh and Vorontsov (2006). This method relies on the autocorrelation function (ACF) and allows one to deduce the diagnostics $\Delta \nu$ and $\delta \nu$ without being capable to derive the individual mode frequencies. The ACF is defined as the Fourier spectrum of a filtered Fourier transform of the time series, where the choice of the filter function can be optimized according to the envelope of the observed signal in the PD spectrum, as shown in Fig. 10. Mosser and Appourchaux (2009) provided a formal definition of the ACF and additional details. The ACF method to derive the large and small frequency separations as a way to achieve the mode identification is efficient, relies on the physical properties of the wave behavior in the mode cavities, and allows one to suppress disturbing effects of the noise in the PD spectrum. This is why the ACF is currently being used in frequency analysis pipelines, although échelle diagrams remain visually attractive and insightful.

Any departure from the asymptotic relation given by Eq. (38), such as considering the lowest frequency regime of $\mathrm{p}$ modes, will introduce curvature in the echelle diagrams. This curvature is also found as the star evolves and mixed modes occur, creating "bumps" in the échelle diagram. These phenomena are effectively illustrated in Fig. 13 given by García and Ballot (2019). For a discussion of a doubtful identification for the CoRoT F-type pulsator HD 49333 due to mode bumping, and how to treat this "doubt" in the context of a Bayesian prior, see Appourchaux (2014).

\section{Mode identification from rotationally split multiplets}

Rotational splitting following Eq. (44) gives rise to multiplet structures in the data: $l=1$ triplets, $l=2$ quintuplets, etc. This has long been known from ground-based data of selfexcited modes and is also observed from space photometry (Kurtz et al., 2014; Pápics et al., 2014; Reed et al., 2014). The detection of complete multiplets with an odd $(2 l+1)$ number of components as in these examples immediately reveals the $l$ and $m$ values of the modes from the Fourier transform of the data. Rotational splitting also gives information on $\Omega(r)$, as discussed by Aerts, Mathis, and Rogers (2019), which includes illustrations based on Kepler data.

\section{Mode identification from period-spacing patterns}

Period spacings $\Delta P$ for low-degree zonal gravitoinertial modes of main-sequence F-type stars typically range from 2000 to $4000 \mathrm{~s}$ for dipole modes and from 1000 to $2500 \mathrm{~s}$ for quadrupole modes. These ranges are obtained when varying the mass (from $1.3 M_{\odot}$ to $2.0 M_{\odot}$ ), age, metallicity, and mixing properties of models in appropriate regimes according to the observed mode frequencies (Van Reeth, Tkachenko, and 
Aerts, 2016). Main-sequence B-type g-mode pulsators, on the other hand, reveal a much broader range covering roughly $\Delta P \in[1000,15000]$ s (Pápics et al., 2017; Szewczuk and Daszyńska-Daszkiewicz, 2017). These stars have masses between $3 M_{\odot}$ and $10 M_{\odot}$.

Gravity-mode period-spacing patterns such as those shown in Figs. 11-13 immediately reveal the sign of $m$. Indeed, prograde modes have a $(P, \Delta P)$ pattern with a downward trend (Fig. 11), while the pattern of retrograde modes reveal an upward trend (Figs. 12 and 13). The slope of these patterns allows estimation of the rotation frequency in the region where the g-mode kernels $K_{n l}(r)$ are dominant. As shown in Fig. 9, the kernels of high-order g modes are sharply peaked near the convective core. Hence, observed gravitoinertial g modes, Rossby modes, and Yanai modes allow $\Omega_{\text {core }}$ to be assessed from the slope of the period-spacing patterns by exploiting the relationship between the observed series of $\lambda_{l m s}$ and $\Pi_{0}, l$ (or $k$ for Rossby or Yanai modes), and $m$. Indeed, $\lambda_{l m s}$ depends on the spin parameter and the value of the asymptotic period spacing, as revealed by Eq. (49). Slightly different methods to identify the mode numbers $l$ (or $k$ ) and $m$, along with an estimation of $\Omega_{\text {core }}$, were developed by Van Reeth, Tkachenko, and Aerts (2016) based on model grids with varying $M, Z, X_{\mathrm{ini}}$, and $D_{\mathrm{ov}}$, and by Ouazzani et al. (2017) and Christophe et al. (2018) based on stretching the $\Delta P$ patterns to obtain $\Pi_{0}$. This stretching is done by searching the value of $\lambda$ such that $\sqrt{\lambda} P_{l m s}^{\text {co }}$ are equally spaced by $\Pi_{0}$. Both methods give excellent agreement on the estimation of $\Omega_{\text {core }}$; see Ouazzani et al. (2019), Fig. 5. Takata et al. (2020) came up with yet another tool for mode identification. It is based on a diagram in which the frequency is plotted against the square root of the frequency. This allows one to identify prograde sectoral modes and deduce at once the average rotation rate and $\Pi_{0}$, which is in line with the numerical method used by Van Reeth, Tkachenko, and Aerts (2016) that delivers mode identification along with estimation of $\Omega_{\text {core }}$ and $\Pi_{0}$.

As discussed earlier, Rossby modes are always retrograde in the inertial reference frame of an observer. These modes occur at similar radial order ( $n$ typically between -10 and -80) (Li et al., 2020) but have higher spins (Aerts, Van Reeth, and Tkachenko, 2017) (values between 15 and 30) than gravitoinertial g modes ( $n$ roughly between -10 and -100 and spins between 1 and 15). Saio, Kurtz et al. (2018) studied the observational appearances of even and odd Rossby modes by computing their visibilities. It was found that the amplitude distributions of odd $(k=-1)$ modes are located at lower frequencies than those of even $(k=-2)$ modes for any given $m$ and that the amplitudes decrease strongly as $m$ increases; see their Fig. 4. These theoretical predictions offer a good way to identify the wave numbers $(k, m)$ for these modes.

\section{Asteroseismic modeling using mode frequencies}

\section{Some modeling preliminaries}

Seeking agreement between the detected identified oscillation mode frequencies $\omega_{n l m}^{\mathrm{obs}, i} \pm \sigma_{\omega_{n l m}^{\mathrm{obs}, i}}$ and those predicted by equilibrium models $\omega_{n l m}^{\text {theo, } i}$ for $i=1, \ldots, N_{\omega}$, with $N_{\omega}$ the number of detected identified oscillation frequencies, constitutes a multivariate (nonlinear) regression problem. Fitting these identified frequencies can generally be done with or without the addition of other seismic diagnostics (such as mean frequency separations, frequency ratios, or other combinations for particular modes) or by adding other observables into the fitting process $\left[T_{\text {eff }}, \log g, \log \left(L / L_{\odot}\right)\right.$, an interferometrically deduced $R_{\star}$, a dynamical binary component mass $M_{\star}$, etc.]. In general, we consider an observed vector $Y^{\mathrm{obs}}$ consisting of $i=$ $1, \ldots, N_{\omega}+M$ components $Y_{i}^{\text {obs }}$ derived from $N_{\omega}$ observed and identified oscillation frequencies and $M \geq 0$ additional observational constraints. Comparison of $Y^{\text {obs }}$ with the corresponding $Y^{\text {theo }}$ predicted by model computations is an extremely powerful method to determine the interior and global properties of stars, including their rotation, mixing, and composition profiles as well as their mass, radius, bulk metallicity, and age. Nonradial oscillations occur in different types of stars in almost all phases of stellar evolution; cf. Fig. 1. This, along with the availability of long-duration high-precision space photometry, has turned the potential of an asteroseismic calibration of the theory of stellar structure and evolution into a reality. The level of sophistication adopted for asteroseismic modeling is highly variable. Here we summarize methodology that can handle the challenging case of pulsating stars having a convective core and rotating up to considerable fraction of their critical rate.

In our description of asteroseismic modeling via regression, we follow the notations and concepts given by Aerts et al. (2018b); i.e., we denote equilibrium models generically as $\mathcal{M}(\boldsymbol{\theta}, \psi)$, where $\boldsymbol{\theta}$ stands for the vector containing the free parameters to be estimated for the fixed choices of the input physics $\psi$ (i.e., frozen microscopic and macroscopic input physics). The goal is to fit as closely as possible the observed and identified oscillation frequencies and other observables by theoretical values derived from the 3D perturbation of $\mathcal{M}(\boldsymbol{\theta}, \psi)$. We keep in mind the following important aspects:

(1) Theoretically predicted oscillation mode frequencies have uncertainties $\sim 0.001 \mathrm{~d}^{-1}(\sim 0.01 \mu \mathrm{Hz})$ due to limitations in our knowledge of physics and due to numerical implementations. The observed oscillation frequencies from space asteroseismology are typically one to several orders of magnitude more precise than the theoretical predictions; cf. Table 1 given by Aerts, Mathis, and Rogers (2019).

(2) The components of $\boldsymbol{Y}^{\text {theo }}$ are strongly correlated.

(3) The components of $\boldsymbol{Y}^{\text {theo }}$ and $\boldsymbol{Y}^{\text {obs }}$ may have significantly different variances, i.e., heteroscedasticity has to be included in the formalism.

(4) The components of $\boldsymbol{\theta}$ may also be strongly correlated. These four properties result in a challenging modeling problem. Indeed, the theoretical uncertainties stemming from limitations in $\boldsymbol{\psi}$ and from numerical implementation to compute the equilibrium models dominate over the measurement uncertainties. This fact is often ignored in the modeling procedure. Moreover, the correlated nature of the fitting problem implies that the uncertainty regions for the parameters in $\boldsymbol{\theta}$ tend to be of multidimensional elongated shape, and therefore hard to interpret. We provide a mathematical scheme that takes into account these challenges.

The limitations of the equilibrium models are due to restrictions to nonrotating $1 \mathrm{D}$ models, missing atomic physics, poor opacities, imperfect numerical schemes to solve the 
differential equations, etc. Some of the distributions of systematic uncertainties in the theoretical predictions of oscillation frequencies are shown in Figs. 2-10 given by Aerts et al. (2018b) for the case of $\mathrm{g}$ modes in stars with a convective core. An assessment of some of the systematic biases in the case of solarlike oscillations was provided by Gruberbauer et al. (2013). From the viewpoint of improving stellar structure and evolution theory, the aim is to select the most likely physical model $\mathcal{M}(\boldsymbol{\theta}, \boldsymbol{\psi})$ from an unbiased sample of stars without introducing a priori bias by restricting too narrowly the choice of the input physics $\boldsymbol{\psi}$.

The general procedure of asteroseismic modeling of an ensemble of stars is graphically depicted in the flowchart in
Fig. 16. We discuss this framework in the rest of this section, but it is not necessary to digest the details of this flowchart to understand the applications treated in Sec. IV.

\section{Setup of the modeling approach}

In the stellar modeling problem at hand, the choices of $\boldsymbol{\theta}$ and $\boldsymbol{\psi}$ are different for stars with a radiative versus a convective core. Moreover, the probing power of $\mathrm{p}$ and $\mathrm{g}$ modes is different; cf. Fig. 9. Hence the choice of the parameters in the vector $\boldsymbol{\theta}$ to be estimated and the level of redundancy in the observables to be used also differ; see Angelou et al. (2017) for a discussion on this topic. While the

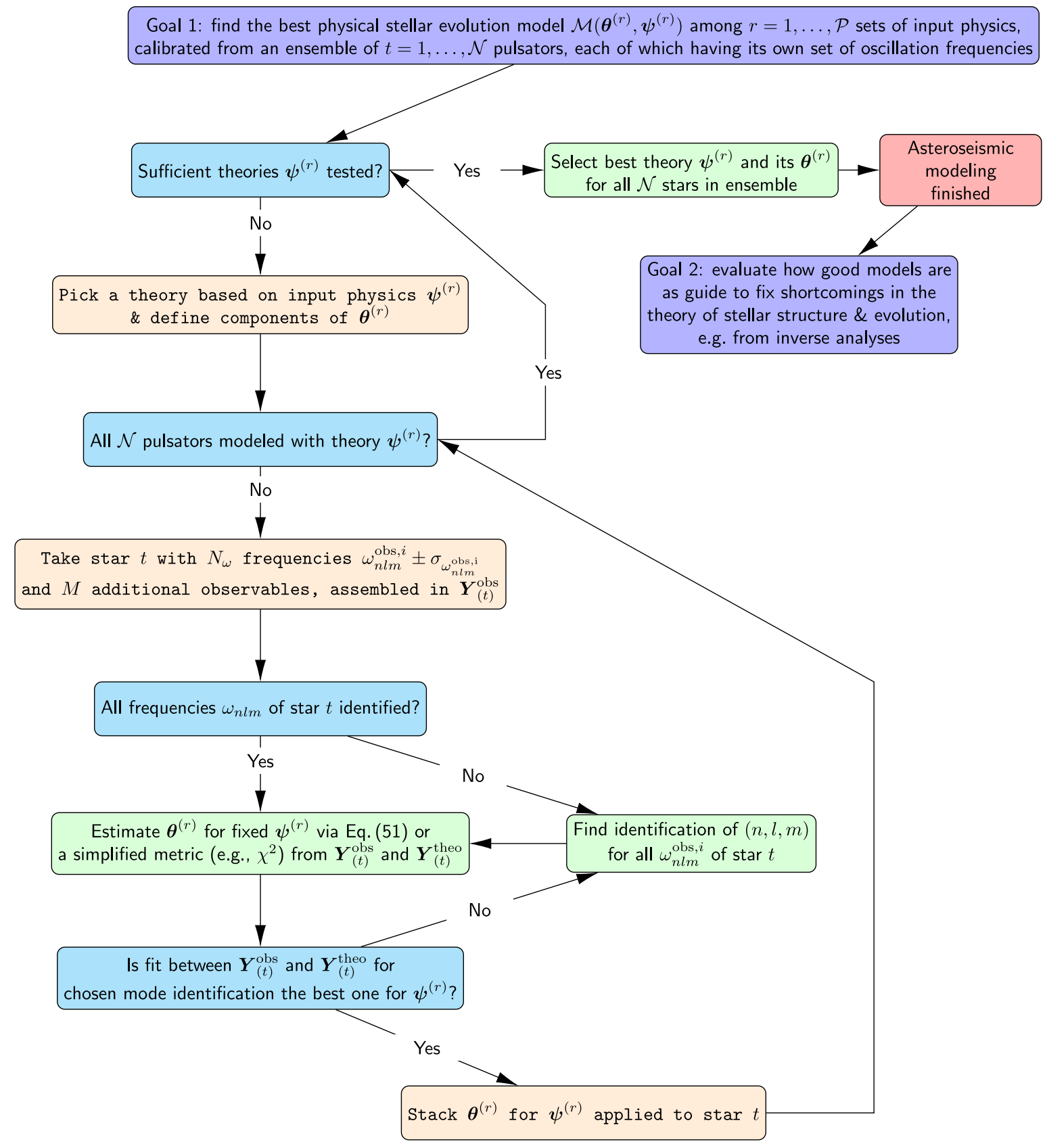

FIG. 16. Schematic representation of the procedure of asteroseismic modeling for an ensemble of stars that summarizes the steps discussed in Sec. III.C. See the text for the meaning of the notation. The green boxes involve statistical methods in the topics of maximum likelihood estimation, pattern recognition, and model selection. 
observed mode frequencies in slowly rotating stars can be condensed into a few "observables" derived from asymptotic approximations as in Eq. (38) or (41), this is not the case for moderate to fast rotators because their patterns depend on the interior rotation and are different for different $l$ and $m$, as revealed by Eq. (49). Even though a stretching method has been devised to transform the observed period-spacing patterns due to $g$ modes into one observable (Christophe et al., 2018), its value depends strongly on $D_{\text {ov }}$ and $Z$ (Mombarg et al., 2019). One can therefore not assume that the frequencies of $g$ modes can be transformed into simple "summary" diagnostics as in the case of solarlike oscillations in slow rotators (where $\Delta \nu$ and $\nu_{\max }$ play that role). To treat asteroseismic modeling in general terms, including applications to hybrid pulsators with both $\mathrm{p}$ and $\mathrm{g}$ modes, we consider a formulation for the matching of individual mode properties. We denote it here for the frequencies, but it can be done for any observable (e.g., period spacings).

Asteroseismic modeling of an individual star is done from an observed vector $\boldsymbol{Y}^{\text {obs }}$, which includes a set of $N_{\omega}$ observables based on the observed frequencies $\omega_{n l m}^{\text {obs, } i}$, where each mode has its own mode cavity, lifetime, and probing power for the interior physics; see Fig. 9. Moreover, one often chooses to add $M$ nonseismic observables to the modeling, depending on their capacity to assess the models and their correlation with respect to other components already chosen for $\boldsymbol{Y}^{\text {obs }}$. In setting up the problem to solve, keep in mind that several observables may be measured independently from each other and occur without covariance, while they do not provide extra information about the star. Singular value decomposition (SVD) methods, among them principal component analysis (PCA), are therefore useful techniques to reduce the dimensionality or to plan (or not plan) follow-up data once asteroseismic information has been deduced. A SVD approach was introduced for helioseismic inversions by Christensen-Dalsgaard and Thompson (1993), while PCA applications for solarlike and g-mode oscillations were done by Angelou et al. (2017) and Mombarg et al. (2019), respectively.

The length of the vector $\boldsymbol{Y}^{\mathrm{obs}}$ is in general star specific and each of its components is accompanied by its own error measurement $\epsilon_{i}^{\mathrm{obs}}$, for which we assume normality, i.e., $\epsilon_{i}^{\mathrm{obs}} \sim \mathcal{N}\left(0, \sigma_{i}^{2}\right)$, as justified by Gruberbauer et al. (2013), Appourchaux (2014), and Aerts et al. (2018b). For each pulsator in an ensemble of stars, the aim is to find the value for $\boldsymbol{\theta}$ that best predicts the observables $\boldsymbol{Y}^{\mathrm{obs}}$, with corresponding value $\boldsymbol{Y}_{0} \equiv \boldsymbol{Y}_{0}\left(\theta_{0}, \boldsymbol{\psi}\right)$. We need to select $\theta_{0}$ such that the distance between $Y^{\text {obs }}$ and $\boldsymbol{Y}^{\text {theo }}$ is minimal for $\boldsymbol{\theta}=\boldsymbol{\theta}_{0}$, keeping in mind the correlated nature of the parameters and the observables, as well as any systematic uncertainties in the theoretical predictions. In such a case, a natural merit function to minimize for the estimation of $\boldsymbol{\theta}$ is the Mahalanobis distance (Johnson and Wichern, 2007). This merit function represents a generalized distance. It has been introduced in stellar variability classification studies for CoRoT data (Debosscher et al., 2007). In the current context of asteroseismic modeling, it takes the form

$\boldsymbol{\theta}_{0}=\arg \min _{\boldsymbol{\theta}}\left\{\left[\boldsymbol{Y}(\boldsymbol{\theta})-\boldsymbol{Y}^{\mathrm{obs}}\right]^{\top}(V+\Sigma)^{-1}\left[\boldsymbol{Y}(\boldsymbol{\theta})-\boldsymbol{Y}^{\mathrm{obs}}\right]\right\}$, where $V=\operatorname{var}(\boldsymbol{Y})$ is the variance-covariance matrix of the vector $\boldsymbol{Y}(\boldsymbol{\theta}, \psi)$ and $\Sigma$ is the matrix with diagonal elements $\sigma_{i}^{2}$ for $i=1, \ldots, N_{\omega}+M$. The notation $X^{\top}$ stands for the transpose of $X$. The matrix $V$ can be estimated so as to capture the variance for each of the components of the theoretically predicted vector $\boldsymbol{Y}^{\text {theo }}$, keeping in mind that the uncertainties due to the limitations of the input physics $\psi$ are much larger than the measurement errors, and that correlations among the vector components occur. The components of $\boldsymbol{Y}^{\text {theo }}$ must cover an appropriate range due to the free parameter ranges of $\boldsymbol{\theta}$. For these reasons, the matrix $V$ can be assessed from grids of models $\mathcal{M}(\boldsymbol{\theta}, \boldsymbol{\psi})$ covering a broad range of $\boldsymbol{\theta}$ for various $\boldsymbol{\psi}$, as illustrated by Aerts et al. (2018a) for the case of $\mathrm{g}$ modes. The Mahalanobis distance defined by Eq. (51) provides a more sophisticated merit function than the often used $\chi^{2}$ based on an Euclidian distance, because it takes into account the variance-covariance structure connected with $\boldsymbol{Y}^{\text {theo }}$ and uncertainties stemming from the limitations of $\boldsymbol{\psi}$. It also considers the overall correlated nature of $\boldsymbol{\theta}, \boldsymbol{Y}^{\text {theo }}$, and their interconnection.

Asteroseismic modeling has thus far mostly been done from minimizing a $\chi^{2}$ merit function relying only on the measurement uncertainties $\epsilon_{i}^{\text {obs }}$. This was improved upon by Gruberbauer, Guenther, and Kallinger (2012) by taking into account unknown systematic uncertainties of $\boldsymbol{Y}^{\text {theo }}$ in a Bayesian framework, using a $\chi^{2}$ formulation. The advantage of minimization as in Eq. (51) is that it allows for heteroscedasticity in and correlation structures among the components of $\boldsymbol{Y}^{\text {theo }}$. Minimizations by Eq. (51) and $\chi^{2}$ were compared by Aerts et al. (2018b), Table 3 and Fig. 12, for a case of g-mode asteroseismology of a SPB star, leading to a somewhat different best solution for $\boldsymbol{\theta}$. Care must be taken when estimating $\boldsymbol{\theta}_{0}$ due to the correlated nature of $\boldsymbol{Y}^{\text {theo }}$ and $\boldsymbol{\theta}$, keeping in mind systematic biases in the theory of stellar interiors and ensuring that $V$ is of proper rank.

Estimation of the uncertainty regions for the components of $\boldsymbol{\theta}_{0}$ is hard to achieve if only one star is modeled, even if many identified frequencies and high-precision classical observables are jointly included in $\boldsymbol{Y}^{\text {obs }}$. This is due to $\boldsymbol{\theta}$ being of high dimension and containing correlated vector components, as stressed by Angelou et al. (2017) and Aerts et al. (2018b). The multi-D error regions are usually elongated. In such a case, inference on the errors of $\boldsymbol{\theta}_{0}$ is conveniently achieved from a MCMC approach; see Foreman-Mackey et al. (2013) for a popular tool used in astronomy. For such applications, clever ways to sample are in order to avoid getting stuck in too few local minima in the case of strong covariances (Handberg and Campante, 2011). For this reason, nested sampling in a Bayesian setting is often considered (Corsaro and De Ridder, 2014). A practical MCMC application to asteroseismology of $\alpha$ Cen A was made by Bazot, Bourguignon, and Christensen-Dalsgaard (2012).

\section{Considering individual stars and ensembles}

A major challenge for low-mass stars with a convective outer envelope is to deal with the 1D treatment of this envelope in the equilibrium models. The outer boundary condition adopted to compute the equilibrium model should 
come from a proper 3D and time-dependent treatment of convection, while it is usually simplified with a timeindependent mlt and a 1D atmosphere model. This aspect of asteroseismic modeling for stars with damped modes excited by turbulent convection is known as the problem of the "unknown surface effects." Clever ways to deal with this, with the attitude of getting rid of the problem, have been developed by using specific combinations of mode frequencies, such as ratios of frequency separations (Roxburgh and Vorontsov, 2003). These ratios were shown to have probing power for the deeper layers of the star while being less sensitive to the physics in the outer layers. In the case of a star with a radiative envelope, the challenge for the modeling is not so much the outer boundary, for which the simple approximation of an Eddington gray atmosphere is fine, but rather how to deal with the near-core boundary mixing and for the high-mass stars also with mass loss due to a radiatively driven and possibly dynamical wind (for $M>15 M_{\odot}$ ).

In the case of solarlike oscillations in low-mass stars, the input physics $\psi$ of the models $\mathcal{M}(\boldsymbol{\theta}, \boldsymbol{\psi})$ is often taken to be similar to that of solar models calibrated from helioseismology. This is fine because one can rely on the reasonable assumption that such stars adhere to similar physics as the Sun, given that they are slow rotators with an extended convective outer envelope. In that case, one can limit the estimation to the minimal set of free parameters to compute the equilibrium models $\boldsymbol{\theta}=\left(M_{\star}, X_{\text {ini }}, Y_{\text {ini }}, \tau\right)$. More sophisticated applications based on machine-learning techniques treating higher dimensions, e.g., by including $\alpha_{\mathrm{mlt}}$ and $D_{\text {mix }}$, are done as well (Bellinger et al., 2016).

For intermediate- and high-mass stars, $\boldsymbol{\theta}$ is always more than four dimensional due to non-negligible interior rotation, core overshooting, and envelope mixing. Even when one can ignore rotation in the computation of the oscillation modes, one deals with a higher-dimensional problem than with low-mass stars. For pulsators with a convective core, the MLE has to be done minimally with $\boldsymbol{\theta}=\left(M_{\star}, X_{\mathrm{ini}}, Z_{\mathrm{ini}}, D_{\mathrm{ov}}, D_{\text {mix }}, \tau\right)$ in the case where rotation can be ignored ( Moravveji et al., 2015). When dealing with gravitoinertial or Rossby modes, i.e., beyond the perturbative treatment of rotation, an estimation of $\Omega(r)$ has to be included in $\boldsymbol{\theta}$, increasing dimensionality even further; see Moravveji et al. (2016) and Van Reeth, Tkachenko, and Aerts (2016) for examples.

The multidimensional uncertainty regions of $\boldsymbol{\theta}_{0}$ are hard to determine for such a complex modeling problem; see Johnston, Tkachenko et al. (2019) for a detailed discussion. It is also challenging to discriminate among candidate theories $\boldsymbol{\psi}$ from the modeling of only one or a few stars. This is why applications optimally consider ensembles of stars. In that case, one has the opportunity to derive the error regions for the individual members or to consider one global average error estimate for each of the components of $\boldsymbol{\theta}$ for the entire population. This approach was applied by Silva Aguirre et al. (2017) and Mombarg et al. (2019) to solarlike and $\gamma$ Dor pulsators, respectively.

As graphically shown in Fig. 16, an important aspect of the ensemble modeling is to assess the quality of a collection of candidate theories, i.e., to consider the following:
- observables $\boldsymbol{Y}_{(t)}^{\text {obs }}$ for $t=1, \ldots, \mathcal{Q}$ members of a representative sample of pulsators, and

- theories $\mathcal{M}\left(\boldsymbol{\theta}^{(r)}, \boldsymbol{\psi}^{(r)}\right), \quad r=1, \ldots, \mathcal{P}$, each of which delivering predicted values $\boldsymbol{Y}_{(r)}^{\text {theo }}$.

In such a setting, the goal is to select the most appropriate theory among the $\mathcal{P}$ candidate theories after applying Eq. (51) or a simplified version of it (e.g., $\left.\chi^{2}\right)$ to every star $t$. This can be done using a grid-based approach where extensive grids of models $\mathcal{M}\left(\boldsymbol{\theta}^{(r)}, \boldsymbol{\psi}^{(r)}\right)$ are computed (Pedersen et al., 2021), or from optimizations "on the fly" via a genetic algorithm approach (Metcalfe et al., 2014), or via Bayesian methods coupled to MCMC (Bazot, Bourguignon, and ChristensenDalsgaard, 2012). Akaike or Bayesian information criteria are proper statistical tools to select the best physical model $\psi$ (Claeskens and Hjort, 2008). Care should always be taken to penalize for higher degrees of freedom when doing the model selection, keeping in mind the dimension of $\boldsymbol{\theta}$.

The ultimate goal of ensemble asteroseismology is to have a pathway to improve the input physics of the theoretical models (indicated as the second goal in Fig. 16). Hence, once the best of the currently available model sets $\psi$ is chosen according to the first goal in the scheme in Fig. 16, one should evaluate how good or bad it represents the data in the details of each of the individual stars in the sample and for the sample as a whole. Stellar models can subsequently be improved from inversion methods originally developed in the framework of helioseismology (Gough, 1985a). Such methods are usually applied on a star-by-star basis once the best 1D model for the appropriate $\boldsymbol{\theta}_{0}$ has been found; see Basu and Chaplin (2017) for a discussion of the methodology. Initial applications of this technique have led to the interior rotation profiles of six subgiants and young red giants (Deheuvels et al., 2014) and in core-helium-burning red giants (Deheuvels et al., 2015). Detailed analyses resulting in profiles $\Omega(r)$ were obtained for the SPB star KIC 10526294 from g-mode triplets (Triana et al., 2015), the red-giant star KIC 4448777 from dipole mixed modes (Di Mauro et al., 2016), and the differential envelope rotation of $16 \mathrm{Cyg}$ A and B by Bazot et al. (2019). Inversion methods have led to an evaluation of the interior structure for $16 \mathrm{Cyg} \mathrm{A}$ and B, revealing discrepancies between the sound speed in the cores of these two stars with respect to those in the 1D models at the level of 5\% (Bellinger et al., 2017), although this binary-exoplanet system is the best calibrated solar ana$\log$ (Davies et al., 2015). Inversions applied to the exoplanet host star Kepler-444 (also known as KOI-3158) resulted in high-precision mass and radius estimates of $M_{\star}=(0.75 \pm 0.03) M_{\odot}, R_{\star}=(0.75 \pm 0.01) R_{\odot}$ and revealed that this star must have had a convective core during the first 8 Gyr of its 11-Gyr lifetime (Buldgen et al., 2019).

\section{APPLICATIONS OF ASTEROSEISMIC MODELING}

At least four reviews and a book have recently been published on the topic of asteroseismic applications based on Kepler or K2 data. Low- or intermediate-mass stars with an outer convective envelope reveal p modes or mixed modes. The solarlike oscillations of these stars were reviewed by Chaplin and Miglio (2013), Hekker and ChristensenDalsgaard (2017), and García and Ballot (2019), and a book 
on the data analysis methodology was written by Basu and Chaplin (2017). We revisit some general results based on solarlike oscillations, focusing on what asteroseismology of such stars can deliver to other fields in astrophysics and on opportunities to improve the theory of stellar interiors. The evolution and $g$ modes of white dwarfs were recently reviewed by Córsico et al. (2019), to which we refer for asteroseismic modeling applications to the stellar remnants of low- and intermediate-mass stars. All of these reviews focused on "fast" modes, i.e., high-frequency modes in the sense that their periods are much shorter than the rotation period of the star. In such a case, the Coriolis force can be ignored or treated using a perturbative approach. For this reason, such applications are relatively easy compared to cases where the rotation and oscillation-mode periods are comparable, demanding a nonperturbative treatment.

We start this section with the simplest applications of asteroseismology and gradually increase the level of complexity, putting more emphasis on applications that have been less summarized in reviews thus far. We focus here on "convenience of use" for the nonexpert while highlighting selected striking results and opportunities to improve stellar physics. The topics of Secs. IV.A-IV.F were chosen without any attempt to be exhaustive for logistical reasons.

\section{A. Sizing, weighing, and aging stars with convective envelopes}

The rotation of stars with $M \lesssim 1.3 M_{\odot}$ slows down efficiently, as first reported by Skumanich (1972) and studied from space photometry (Meibom et al., 2015). Although the details of their rotational evolution are not yet fully understood (Van Saders et al., 2016), this efficient slowdown is interpreted in terms of magnetic braking induced by the dynamo created in their convective envelope and angular momentum loss via a thin stellar wind. These stars, as well as all evolved stars, have extended convective envelopes that are the seeds of stochastic driving of modes by turbulent convection. Chaplin et al. (2014) provided a summary of the asteroseismic properties of the ensemble of dwarfs and subgiants observed with the Kepler spacecraft and found the modes occurring near the frequency of maximum power in the PD spectra to have radial orders ranging from $n=17$ to 19 for dwarfs and from $n=15$ to 19 for subgiants. For stars born with a radiative core $\left(M \lesssim 1.1 M_{\odot}\right)$ and similar metallicity as the Sun, the transition from hydrogen-core to hydrogen-shell burning occurs near $\nu_{\max } \simeq 2000 \mu \mathrm{Hz}$. This transition gradually shifts to lower frequencies for larger and more massive stars. The transition from core to shell burning occurs at $\nu_{\text {max }} \simeq 800 \mu \mathrm{Hz}$ for stars near the upper mass for which solarlike oscillations still occur $\left(M \simeq 1.5 M_{\odot}\right)$.

Basic key ingredients of asteroseismic applications based on solarlike oscillations are the global seismic scaling relations relying on solar values. These were derived prior to space asteroseismology by Kjeldsen and Bedding (1995). These scaling relations are based on the large frequency separation defined in Eq. (39) and the frequency of maximum power $\nu_{\max }$ discussed in Sec. I:

$$
\begin{aligned}
\frac{\Delta \nu}{\Delta \nu_{\odot}} & \approx\left(\frac{M}{M_{\odot}}\right)^{1 / 2}\left(\frac{R}{R_{\odot}}\right)^{-3 / 2}, \\
\frac{\nu_{\max }}{\nu_{\max , \odot}} & \approx\left(\frac{M}{M_{\odot}}\right)\left(\frac{R}{R_{\odot}}\right)^{-2}\left(\frac{T_{\mathrm{eff}}}{T_{\mathrm{eff}, \odot}}\right)^{-1 / 2} .
\end{aligned}
$$

In Eqs. (52), the solar reference values have to be computed using the same methodology as for the star(s) under study to achieve meaningful and consistent results. In their Table 1, Pinsonneault et al. (2018) listed solar reference values for various asteroseismic pipelines in use today. It is seen in Eqs. (52) that the large frequency separation scales with the square root of the mean density of the star. Because one relies on the mass, radius, and oscillations of the Sun for a particular choice of input physics $\boldsymbol{\psi}_{\text {Sun }}$, one has a quick and easy way to deduce the mass and radius of the star under study. This type of stellar weighing and sizing implies a major simplification: none of the steps in the procedure shown in Fig. 16 have to be taken, because one assumes that the input physics to model the Sun $\psi_{\text {Sun }}$ is also valid for the star(s) under study and one does not test any models with other choices for the input physics $\psi$. This does not allow one to perform model selection among candidate theories $\boldsymbol{\psi}$, as one freezes the latter to the solar one calibrated from helioseismology. Moreover, by relying on the solar values $\Delta \nu_{\odot}$ and $\nu_{\max , \odot}$ one implicitly assumes that the star under study has the same metallicity and chemical mixture as the Sun. By using the scaling relations in this way, there is no such thing as asteroseismic modeling in the sense of Fig. 16.

For frozen $\boldsymbol{\psi}_{\text {Sun }}$, it follows from the scaling relations in Eq. (52) that a measurement of $T_{\text {eff }}, \Delta \nu$, and $\nu_{\max }$ suffices to deduce the mass and radius of a star. For such an application, it is not even necessary to know the identification of the individual $\mathrm{p}$ modes, as long as one can estimate $\Delta \nu$ and $\nu_{\max }$ from the PD spectrum, with or without the help from an ACF. This has major applications, as asteroseismic $M_{\star}$ and $R_{\star}$ values can be computed easily for use in various fields of astrophysics, even from short and/or gapped time series. For this reason, substantial effort has been put into testing the scaling relations from independent methods, notably from an interferometric radius, as done by Huber et al. (2012) and White et al. (2013), or from an astrometric radius, as treated by Silva Aguirre et al. (2012), Huber et al. (2017), and Zinn et al. (2019). Overall agreements are excellent for stars with a fairly large range in radii, from about $0.8 R_{\odot}$ to above $10 R_{\odot}$ : this is a tribute to the trio of asteroseismology, astrometry, and interferometry.

Thanks to their simplicity, the scaling relations in Eq. (52) have been used extensively to deduce the masses and radii of stars with solarlike oscillations observed with space photometry. Aside from the recent Kepler catalog papers on dwarfs and subgiants (Chaplin et al., 2014) and red giants (Yu et al., 2018), we refer the interested reader to earlier CoRoT catalogs for red giants by Kallinger et al. (2010) and Mosser et al. (2010, 2012), as well as to the K2 catalog by Stello et al. (2017). The scaling relations were even expanded to the extremely low frequencies of solarlike oscillations in $\mathbf{M}$ giants belonging to the class of semiregular variables; cf. Fig. 1. This allowed researchers to assess and interpret the period-luminosity relations derived from ground-based microlensing 


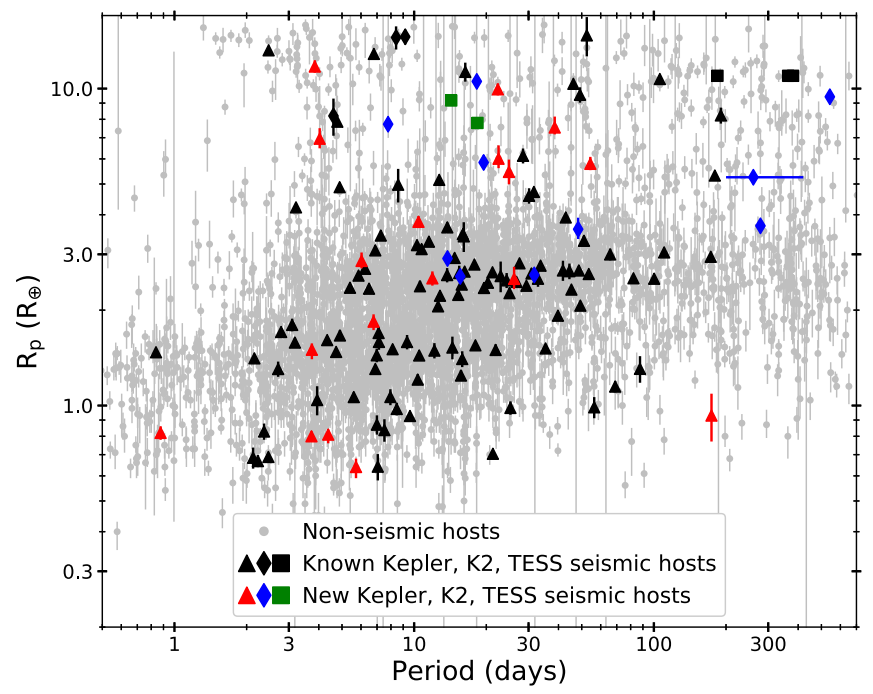

FIG. 17. Planetary radii as a function of orbital period, where the properties of exoplanet host stars with and without asteroseismic estimation are compared. Asteroseismology of the host star not only provides the age of the exoplanetary system but also improves the planetary radii by a factor of $\sim 2$ compared to the case where such data are not available. Adapted from Chontos et al., 2019.

surveys in terms of the interior structure of such highly evolved stars (Mosser et al., 2013). Analyses of M giants observed with Kepler led to the discovery of nonradial oscillations of low inertia in such stars, interpreted as f modes by Stello et al. (2014). This discovery holds major potential and has yet to be explored further. In particular, it paves the way to perform extragalactic asteroseismology from observations of $\mathrm{M}$ giants in the Magellanic Clouds.

As another key application, masses and radii for exoplanet hosts based on the scaling relations have been published in a number of studies, among them Huber et al. (2013), Van Eylen et al. (2014, 2018), Silva Aguirre et al. (2015), Campante et al. (2016a), and Lundkvist et al. (2016). A radius estimate of an exoplanet host star from the scaling relations propagates directly into a radius estimate of its exoplanets for which a transit has been measured. Asteroseismic sizing from scaling relations is therefore particularly convenient for exoplanet studies. Once an exoplanet has been detected in space photometry, the latter can be revisited in an attempt to measure its host star's values of $\Delta \nu$ and $\nu_{\max }$ (Chontos et al., 2019). Figure 17 shows the planet radii versus orbital periods for an assembly of Kepler, K2, and TESS exoplanets, comparing those with and without asteroseismology of the host star. As highlighted in the figure, more measurements of $\Delta \nu$ and $\nu_{\max }$ from the PD spectra are achieved as detection methods are refined and spectroscopy (to estimate $T_{\text {eff }}$ ) are assembled. The gain in precision of the exoplanetary radius is typically a factor of $\sim 2$ when asteroseismic sizing of the host star from scaling relations can be done relative to the case where no oscillations are detected.

Once the mass and radius of a star have been estimated from the scaling relations, its age and hence its evolutionary stage can be assessed. This requires evolutionary models and was originally done for the Sun. Christensen-Dalsgaard (1988) introduced the so-called CD diagram to estimate the age of solar-type stars from their large and small frequency separations. Chaplin et al. (2014) made a thorough analysis to estimate the ages of the more than 500 dwarfs and subgiants observed with Kepler. This ensemble analysis was based on six different data analysis pipelines and 11 stellar model grids in order to assess the combined effect of observational and model uncertainties by taking into account $V+\Sigma$, as discussed after Eq. (51). This is an application of Fig. 16 where one does not use the individual frequencies $\omega_{n l m}^{\mathrm{obs}, i} \pm \sigma_{\omega_{n l m}^{\mathrm{obs}, i}}$ as input, but rather $\boldsymbol{Y}^{\mathrm{obs}}=\left(\nu_{\max }, \Delta \nu, \delta \nu, T_{\mathrm{eff}},[\mathrm{Fe} / \mathrm{H}]\right)$ to estimate the parameters $\boldsymbol{\theta}=(M, R, \tau)$ and quantities that can be derived from these three (such as the mean density and gravity). For this application, Chaplin et al. assumed that the components of $\boldsymbol{Y}^{\text {theo }}$ are not subject to uncertainties and are not correlated with each other, such that $\chi^{2}$ can be used as a merit function. Under these assumptions, this ensemble modeling led to average relative precisions of approximately $5.4 \%$ in mass, $2.2 \%$ in radius, and $\sim 10 \%-20 \%$ in age. Such relative precisions are within reach when spectroscopic estimates of $T_{\text {eff }}$ and $[\mathrm{Fe} / \mathrm{H}]$ are available. If only $\nu_{\max }$ and $\Delta \nu$ are available, the relative precisions are downgraded by about a factor of 2, which is still excellent and often the only way to get an age estimate of isolated stars in the Milky Way.

Bellinger $(2019,2020)$ derived scaling relations for the ages of dwarfs, subgiants, and red giants from $\boldsymbol{Y}^{\text {obs }}=\left(\nu_{\max }\right.$, $\left.\Delta \nu, \delta \nu, T_{\text {eff }},[\mathrm{Fe} / \mathrm{H}]\right)$. For the dwarfs, the relations were deduced from fits to these quantities for 80 stars whose measurement uncertainties for $\delta \nu$ and $\nu_{\max }$ are better than $10 \%$ and $5 \%$, respectively. This leads to age precision estimates of about $10 \%$ for dwarfs. These formulas are easy to use (e.g., for exoplanet host aging), but users have to keep in mind that the relations explicitly rely on the solar input physics via homology relations. Thus, the fact that the interior rotation, mixing, and magnetism of the stars might be different than those of the Sun is ignored.

A major breakthrough in asteroseismology was achieved upon the detection of mixed dipole modes in Kepler data of evolved low-mass stars (Beck et al., 2011; Bedding et al., 2011) after they had been theoretically predicted in the context of CoRoT by Dupret et al. (2009). The mixed modes can have a gravity-dominated or a pressure-dominated character, depending on the extent and shape of their propagation cavity. Such dipole mixed modes in evolved stars occur together with radial and quadrupole $\mathrm{p}$ modes, which obey the asymptotic relation in Eq. (39) and probe the convective envelope of the star. Measurement of $\Delta \nu_{n l}$ and $\Delta P_{n l}$ thus allows one to derive the mass and radius of the star from scaling relations, as well as its evolutionary stage (Bedding et al., 2011). Indeed, the gravity-dominated mixed modes probe the deep stellar interior and have different values for hydrogen-shell-burning red giants than for core-helium-burning red giants. This allows one to deduce the nuclear burning stage of these two types of red giants, while their surface properties are the same. Period spacings of dipole mixed modes lead to higher-precision age estimates than the large frequency separation from $\mathrm{p}$ modes. Moreover, the period spacings are a sensitive probe for internal mixing in intermediate-mass dwarfs and allow one 
to calibrate the core overshooting efficiency using lowluminosity red-giant stars (Hjørringgaard et al., 2017). We return to this capacity for $g$ modes in Sec. IV.E.

Estimation of $R_{\star}$ from asteroseismology combined with a spectroscopic measurement of $T_{\text {eff }}$ allows one to deduce the luminosity of the star and hence derive an asteroseismic parallax (Silva Aguirre et al., 2012). Comparisons between such asteroseismic parallax with the one from Gaia astrometry reveals excellent agreement for dwarfs (De Ridder et al., 2016) and red giants (Huber et al., 2017). This allows one to probe the deep end of the Milky Way with luminous pulsating stars (Mathur et al., 2016). The capacity of joint asteroseismic aging, sizing, and distance estimation opened up the field of galactic archaeology, which had already been jump-started prior to the Gaia era by Miglio et al. (2009). Their study of various populations of core-helium-burning red giants in the galactic disk observed with CoRoT opened a new field of mapping and dating stellar populations from red-giant asteroseismology (Miglio et al., 2013). Meanwhile, extensive progress has been made in archaeological studies for the pointing directions in the Milky Way covered by CoRoT, Kepler, and K2 coupled with large spectroscopic surveys and/ or Gaia data; see Stello et al. (2015), Anders et al. (2017a, 2017b), Serenelli et al. (2017), Stello et al. (2017), Pinsonneault et al. (2018), Sahlholdt and Silva Aguirre (2018), Silva Aguirre et al. (2018), Rendle et al. (2019), Sharma et al. (2019), and Zinn et al. (2019). Major potential for extending this topic toward all-sky coverage is being offered by the ongoing TESS mission (Ricker et al., 2016) and the future PLATO mission (Rauer et al., 2014). These surveys should optimally be coupled with spectroscopic surveys with multiobject spectrographs to target hundreds of thousands of asteroseismically aged and sized red giants. The beginnings of such large-scale asteroseismic archaeology have already revealed abundances and distances that allowed one to separate high- and low- $[\alpha / \mathrm{Fe}]$ populations in the Milky Way disk (Chiappini et al., 2015). In this way, asteroseismology has become a key ingredient in the study of the multiple populations and of the chemical evolution of our Milky Way.

To circumvent computationally intensive age derivation from evolutionary models, age scaling relations were derived by Bellinger (2020). These rely on the asteroseismic properties of 1000 red giants and are convenient for galactic archaeologists. These age relations assume that stars adhere to $\psi_{\text {Sun. }}$. Their quoted precision of $\sim 15 \%$ does not take into account systematic uncertainty due to the unknown evolutionary properties on the main sequence. As highlighted by Fig. 7, uncalibrated descriptions of internal mixing in dwarfs with a convective core are used in the models. Moreover, asteroseismology revealed the theory of angular momentum transport to be limited (Aerts, Mathis, and Rogers, 2019); see also Fig. 6. As long as users of "recipe-type" aging recognize this major culprit stemming from fixing the input physics, the scaling relations are a convenient tool for initial asteroseismic and comparative stellar aging of populations, preventing us from having to go through à la carte modeling, a term introduced by Lebreton and Goupil (2014) and Lebreton, Goupil, and Montalbán (2014) that is represented in Fig. 16. To get the maximum precision out of the data of a particular star, including aging to better than $10 \%$, detailed modeling according to Fig. 16 is in order. Treating populations of $\mathcal{N}$ stars in this way is much more cumbersome than applying age scaling relations, but it is the only way to properly take into account the fact that the internal mixing of stars can be diverse (cf. Fig. 7), even for a population of stars born with the same metallicity and similar rotation; discussed in Sec. IV.E.

\section{B. Assessing sharp features in stellar structure}

Fitting the frequencies or periods of oscillations for individual stars and particularly for an ensemble of stars allows one to learn more about the quality of the input physics $\psi$ of stellar models following Fig. 16. This can be done from fitting some of the individual detected and identified oscillation frequencies and periods, rather than simply using the measured averages of global patterns based on the asymptotic theory as in Eqs. (38) and (39) or Eqs. (41) and (42). An intermediate step between the exploitation of only the average value of the frequency or period spacing and the full-blown fitting of all measured individual oscillation modes is offered by modeling deviations from the expected constant spacings due to so-called structural glitches. Sharp features in the sound speed are called acoustic glitches, while those in the BruntVäisälä frequency are termed buoyancy glitches. These glitches may lead to oscillatory deviations in the patterns of $\mathrm{p}$-mode frequencies or $\mathrm{g}$-mode periods. Interpretation of such measured deviations goes beyond the simple use of scaling relations and provides a good opportunity to derive detailed properties of stellar structure.

Studies of acoustic glitches in the Sun from helioseismology led to the overshoot properties at the base of the solar convective envelope (Monteiro, Christensen-Dalsgaard, and Thompson, 1994) and to the capacity to position the second ionization zone of helium (Monteiro and Thompson, 2005). Following those solar studies, the potential of exploiting measured oscillatory deviations due to acoustic glitches was investigated further by Monteiro, Christensen-Dalsgaard, and Thompson (2000), Basu et al. (2004), and Houdek and Gough (2007) for Sun-like stars covering a mass range of $M_{\star} \in[0.85,1.2] M_{\odot}$. This led to methods to infer the size of the convective envelope and to derive properties of the overshoot transition layer at the bottom of the convective envelope; see Hekker and Christensen-Dalsgaard (2017) for an extensive discussion. This methodology was put into practice for Kepler data of Sun-like stars by Mazumdar et al. (2014). Figure 18 illustrates the measured oscillatory frequency deviations due to acoustic glitches in the structure of 16 Cyg A, following its PD in Fig. 10. In a series of papers exploiting the measured periodic deviations from constant spacings, Verma et al. (2014, 2017) measured the locations of the base of the convection zone and of the helium ionization zones for Sun-like stars observed by Kepler, as well as their helium abundance (Verma et al., 2019) and the level of helium settling due to atomic diffusion (Verma and Silva Aguirre, 2019). In these studies, analytical modeling was compared to numerical asteroseismic modeling following the scheme in Fig. 16 by estimating $\boldsymbol{\theta}=$ $\left(M_{\star}, Y_{\text {ini }},[\mathrm{Fe} / \mathrm{H}], \alpha_{\mathrm{mlt}}, \alpha_{\mathrm{ov}}\right)$ for equilibrium models with and without helium settling (i.e., for two different $\boldsymbol{\psi}$ 's in Fig. 16). The results of such numerical modeling for $16 \mathrm{Cyg} \mathrm{A}$ are shown as a solid line in Fig. 18. This led to $Y_{\text {ini }} \in[0.231,0.251]$, while 


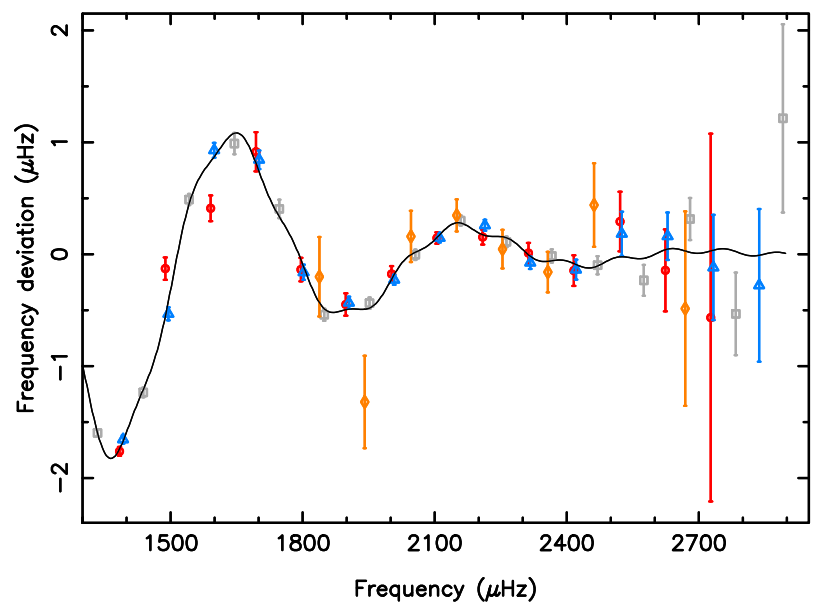

FIG. 18. Frequency deviations with respect to a fourth-order polynomial fit to the measured frequencies for the modes of degree $l=0$ (blue triangles), $l=1$ (gray squares), $l=2$ (red circles), and $l=3$ (orange diamonds) of $16 \mathrm{Cyg} \mathrm{A}$, based on the PD shown in Fig. 10. The full line represents a fit to the oscillatory signal caused by sharp features in the star's sound speed in the second ionization zone of helium. Adapted from Verma et al., 2014.

a similar application to $16 \mathrm{Cyg} \mathrm{B}$ revealed $Y_{\text {ini }} \in[0.218$, 0.266]. The differences in helium mass fraction $\Delta Y$ derived from models with and without helium settling were found to be $\Delta Y \sim 0.038$ for both stars. This result is representative for an additional $~ 30$ Sun-like stars analyzed in the same way. As such, frequency deviations due to acoustic glitches offer a unique way to derive the helium composition of stars too cool to reveal helium spectral lines.

For the solar analogs $16 \mathrm{Cyg} \mathrm{A}$ and B, even more details on the physics of the envelope could be derived, notably latitudinal differential rotation due to a dynamo effect. In an updated study based on the same light curve as the one used by Davies et al. (2015), the measured rotational splitting of 16 Cyg A and B led to envelope rotation rates that are higher at the equator than at the pole. Bazot et al. (2019) found the differences of the rotation frequencies between the equator and the pole to be $320 \pm 269$ and $440 \pm 370 \mathrm{nHz}$ for $16 \mathrm{Cyg}$ $\mathrm{A}$ and $\mathrm{B}$, respectively, while the equatorial rotation frequencies were $535 \pm 75$ and $565 \pm 140 \mathrm{nHz}$. This envelope rotation behavior of both binary components is similar to that of the Sun. The results on turbulence and rotation of this solar-analog binary and exoplanet system illustrate how modeling of individual frequencies provides an opportunity to improve the treatment of envelope convection in cool stars.

Mode trapping due to transition zones with compositional changes is omnipresent in stars at evolved stages. This phenomenon has already been detected from ground-based white-dwarf asteroseismology (Winget et al., 1991, 1994). It was found in large ensembles of Kepler $\gamma$ Dor and SPB stars analyzed by Pápics et al. (2017) and Li et al. (2020), of red giants by Mosser et al. (2015), and of subdwarf B pulsators by Reed et al. (2011), Østensen et al. (2014), Uzundag et al. (2017), and Kern et al. (2018), while it was also confirmed in white dwarfs from K2 photometry (Hermes et al., 2017a). The trapping of $\mathrm{g}$ modes or mixed modes was theoretically investigated for red giants by Cunha et al. (2015), for subdwarfs by Charpinet et al. (2000), and much earlier for white dwarfs in the seminal paper by Brassard et al. (1992). Hence, modeling of mode trapping due to structural glitches can now be done across the entire HRD (Cunha, Avelino et al., 2019).

During the main-sequence phase, buoyancy glitches occur due to the shrinking convective core of intermediate- and highmass dwarfs. These glitches lead to deviations from the constant period spacing of high-order g modes for stars with limited chemical mixing in their radiative envelopes. Such periodic deviations were first observed in a SPB observed with CoRoT (Degroote et al., 2010a). The signature of buoyancy glitches was also found in the period-spacing patterns of $\gamma$ Dor stars (Van Reeth et al., 2015), as illustrated in Fig. 11 for one of them (KIC 11721304). A sharp deviation from a periodspacing pattern can also occur when a pure inertial mode in the convective core of a rapid rotator couples resonantly to a heatdriven gravitoinertial mode in the radiative zone (Ouazzani et al., 2020). Such resonances appear at specific mode frequencies. For evolved dwarfs with a shrinking convective core, the signal due to sharp features in the structure of the equilibrium model or due to resonances with inertial modes may be intertwined with mode bumping or avoided crossings; see Smeyers and Van Hoolst (2010) for a mathematical description of these phenomena. A single resonance between a gravitoinertial mode in the envelope and a pure inertial mode in the core, or a single avoided crossing, leads to just one dip in the period-spacing pattern, as observed in Fig. 12. A $\mu$ gradient zone in the near-core region instead gives rise to recurring quasiperiodic deviations. Modeling of such regular deviations provides an excellent opportunity to derive properties of $D_{\text {mix }}(r)$ in the deep stellar interior. Analytical predictions for the oscillatory deviations due to a receding convective core were derived for high-order $\mathrm{g}$ modes of nonrotating $\gamma$ Dor and SPB stars by Miglio et al. (2008). Bouabid et al. (2013) generalized this to a numerical framework for rapidly rotating $\gamma$ Dor stars. These theoretical studies have been put into practice for dwarfs to estimate their levels of chemical mixing from asteroseismic modeling following Fig. 16, with adopted $D_{\text {mix }}(r)$ profiles as in Fig. 7 . We discuss such recent applications in Sec. IV.E.

Moving beyond the main sequence, periodic components in the oscillation frequencies due to sharp features in the convective envelope of the red giant HR 7349 were found in its CoRoT data. The signal is due to a local depression of the sound speed in the second ionization zone of helium (Miglio et al., 2010). Similar studies from Kepler observations of red giants were summarized by Hekker and ChristensenDalsgaard (2017). A powerful analysis to derive the helium abundance for red giants was presented by McKeever, Basu, and Corsaro (2019) for an ensemble of 27 pulsating red-giant branch (RGB) stars in the metal-rich open cluster NGC 6791 observed by Kepler. The helium abundances and ages for each individual cluster RGB star were combined to create a distribution for the cluster's age and helium abundance, resulting in $Y_{\text {ini }}=0.297 \pm 0.003$ and $\tau=8.2 \pm 0.3$ Gyr. Such precision for aging and helium abundance determination is beyond the reach of classical cluster studies involving 
isochrone fitting (An et al., 2007), even in the Gaia era (Bossini et al., 2019).

Dipole mixed modes in red giants offer additional opportunities. Structural glitches and mode trapping result in deviations from the asymptotic relations of period spacings of $\mathrm{g}$ modes as in Eq. (41), or of gravitoinertial modes as in Eq. (49). Mixed modes comply with a more complex asymptotic spacing pattern due to their mixed g- and p-mode nature. Red giants are slow enough rotators to ignore the Coriolis force for the derivation of their period-spacing expression, of which Eqs. (38) and (41) are the limiting cases for pure pressure and pure gravity modes, respectively. The asymptotic expression for dipole mixed modes depends on the evanescent zone between the g-mode cavity determined by buoyancy as the dominant restoring force and the p-mode cavity where the pressure force is the dominant restoring force. The location, shape, and width of this evanescent zone all play a role in the coupling between these two cavities. The coupling factor $q$ reaches extreme values of zero for no coupling and 1 for full coupling. The asymptotic expression for mixed dipole modes was discussed by Unno et al. (1989). Generalized versions and their use in space asteroseismology were given by Mosser et al. (2014), Takata (2016a, 2016b), and Pinçon, Goupil, and Belkacem (2019). Observational estimations of $q$ from Kepler data were made by Buysschaert et al. (2016), Mosser, Pinçon et al. (2017), Hekker, Elsworth, and Angelou (2018), Jiang, Christensen-Dalsgaard, and Cunha (2018), and Mosser et al. (2018). Hekker, Elsworth, and Angelou (2018) used a description that explicitly relies on the radial order of the modes and allows one to constrain the pmode and g-mode frequency or period offsets observationally. This work revealed the g-mode period offsets to correlate with the core boundary for RGB stars, while the p-mode offsets for core-helium-burning stars require additional mixing that is in line with the suggestions by Constantino et al. (2015) and Bossini et al. (2017). Hekker, Elsworth, and Angelou (2018) also found that $\ln q$ relates linearly to the width of the evanescent zone normalized by its position. These findings provide observational guidance to tweak $N(r)$ and deduce the core mass of red giants in various evolutionary stages. Pinçon, Goupil, and Belkacem (2019) demonstated that $q$ is tightly connected with the width of the evanescent zone and showed how this zone changes when stars evolve from the subgiant to the RGB and further toward the red clump. This study highlighted the capacity of $q$ to probe the dynamics of the zone between the hydrogen-burning shell and the bottom of the convective envelope. To date this analytical work has focused on stars with masses below $1.2 M_{\odot}$. It needs to be extended to stars born with a well-developed convective core for one to understand the full structural and evolutionary properties of the evanescent zone for such stars, including those with $M_{\star} \gtrsim 2 M_{\odot}$, which will end up in the secondary clump.

\section{Improving the physics of cool-star surface convection}

In the case of low-mass stars, one can go beyond the analytical modeling of acoustic glitches, which is in itself an improvement over the use of scaling relations. A major aim of asteroseismology is to let go of the assumption that low-mass stars adhere to $\boldsymbol{\psi}_{\text {Sun }}$. This can be done by comparing the choice of the solar input physics with any other choice of $\boldsymbol{\psi}$ from evaluations between predicted and measured values of the individual oscillation frequencies. Modeling according to Fig. 16 then requires one to "overcome" the surface effects in the case of cool stars with a convective envelope. Indeed, for those stars, the adiabatic approximation of the oscillation frequencies and the use of simplified boundary conditions based on 1D atmosphere models are not good enough relative to reality, as such approaches lead to inappropriate frequency predictions $\omega_{n l m}^{\text {theo, } i}$. Relying on equilibrium models with model atmospheres based on mlt to describe the envelope convection rather than taking full account of the turbulent pressure in the superadiabatic near-surface regions leads to $\mathrm{p}$-mode frequencies that are too high (Christensen-Dalsgaard, 2002). The offset is larger for higher radial orders, i.e., for higher mode frequencies. For the solar oscillations, this leads to offsets for $\Delta \nu_{\odot}$ of the order of $10 \mu \mathrm{Hz}$. Similar offsets are expected to occur for the oscillations of all Sun-like stars with solarlike oscillations. The values of the offsets are much larger than typical uncertainties of the measured oscillation frequencies. The surface effects must hence be assessed to prevent errors in the estimation of $\boldsymbol{\theta}$, even for fixed $\psi_{\text {Sun }}$, when going through Fig. 16.

Methods have been devised to "correct for" or "minimize" the unknown surface effects, guided by their properties regarding the Sun. One way to deal with the surface effects was originally proposed by Roxburgh and Vorontsov (2003), who came up with combinations of $\mathrm{p}$-mode frequencies (the so-called $r_{02}$ and $r_{01}$ indices) that suppress the sensitivity to the outermost layers, decreasing in this way the influence of the limitations of the 1D models in the asteroseismic modeling. More complex indices were subsequently defined with the same aim (Roxburgh, 2005). Several other methods to fit the surface effects with a statistical model, with the aim of "getting rid" of the differences between the measured and model frequencies, were developed. These adopted various levels of sophistication in the fitting following the pioneering paper by Kjeldsen, Bedding, and Christensen-Dalsgaard (2008). Ball and Gizon (2014, (2017), Basu and Kinnane (2018), Compton et al. (2018), and Jørgensen and Weiss (2019) offered ways to treat the surface effects.

Even with the simple pragmatic approach taken by Kjeldsen, Bedding, and Christensen-Dalsgaard (2008), the gain between modeling based on the scaling relations versus fitting the actual frequencies is roughly a factor of 2 in the precision of the mass, radius, and age. Such asteroseismic modeling was done for the CoRoT exoplanet host HD 52265 by Lebreton and Goupil (2014), showcasing how aging (and weighing and sizing) of the host star by individual oscillation frequency fitting can be done at the level of $10 \%$. While this study was applied to an individual CoRoT target star according to the principles of Fig. 16, Lund et al. (2017) and Silva Aguirre et al. (2017) applied this full scheme to an ensemble of 66 stars observed in 1-min cadence Kepler photometry with a time base of up to $4 \mathrm{yr}$ in the so-called legacy sample of the mission. This study covered the following values for $\theta$ : stellar masses between $0.8 M_{\odot}$ and $1.6 M_{\odot}, \quad Y_{\text {ini }} \in[0.2,0.4]$, $Z_{\text {ini }} \in[0.0025,0.05], \alpha_{\text {conv }} / \alpha_{\odot} \in[0.5,1.3]$, and ages between 
1 and 12 Gyr. Silva Aguirre et al. (2017) considered seven different choices for the input physics $\psi$, all of which are nonrotating, nonmagnetic 1D models that ignore radiative levitation in the treatment of the microscopic atomic diffusion; see their Table 1. All pulsation computations were done in the adiabatic approximation and ignored the Coriolis and Lorentz forces. The asteroseismic modeling led to average relative uncertainties of $2 \%$ in radius, $4 \%$ in mass, and $10 \%$ in age and revealed degeneracies between the stellar mass $M_{\star}$ and initial helium abundance $Y_{\text {ini }}$. All seven adopted $\psi$ led to comparable fit quality when considering calibrations from the Sun, angular diameter measurements, Gaia parallaxes, and binarity. An initiative to assess the differences in stellar models computed with various evolution codes by adopting the same input physics with the aim of evaluating the level of numerical uncertainties and comparing them to asteroseismic uncertainties was provided by Christensen-Dalsgaard et al. (2020) and Silva Aguirre et al. (2020), where the results may be found. Such activities are an important aspect of the model uncertainties at play when one performs modeling via Fig. 16.

Currently the focus in asteroseismology of low-mass stars adopts a true asteroseismic spirit: rather than shifting the measured frequencies to remove the surface effects, the latter are considered an "observational gift" to improve the weaknesses in the physics of 1D models. This shift in spirit of the asteroseismologists rather than in the measured oscillation frequencies offers great potential. Indeed, the asteroseismic modeling of stars with an outer convective envelope can be further improved by using the measured surface effect as an opportunity rather than a nuisance. A common procedure adopted in 1D models of low-mass stars is to calibrate an interior model and an atmosphere model so as to be consistent with the Sun for a single value of $\alpha_{\mathrm{mlt}}$ and do the stitching of the two deep enough in the adiabatic part of the atmosphere. However, this procedure has limitations for evolved stars (Choi et al., 2018). Asteroseismology of a variety of stars permits one to do better. Indeed, the shift between the measured oscillation frequencies and those predicted by $3 \mathrm{D}$ hydrodynamical simulations of convection relevant for the outer envelopes of low-mass stars is informative when evaluating such simulations (Zhou, Asplund, and Collet, 2019), assessing nonadiabatic stability analyses (Houdek et al., 2019), and finding out how to "patch" 3D atmosphere models to 1D models of stellar interiors.

Following the initial achievement to match a 3D atmosphere model to a 1D solar interior model by Rosenthal et al. (1999), detailed studies were conducted to achieve optimal patching. The measured surface terms from helioseismology by Magic and Weiss (2016) and from asteroseismology by Sonoi et al. (2015), Ball et al. (2016), and Trampedach et al. (2017) were exploited from the patching of 3D atmosphere models to $1 \mathrm{D}$ interior models. This led to improved boundary conditions based on 3D convection simulations. Guidance from measured surface effects and adiabatic predictions of solarlike oscillation frequencies was used to derive the optimal connection depth in the atmosphere. Although this approach does not yet take into account nonadiabatic effects, it offers good potential to come to a better treatment of the $3 \mathrm{D}$ simulations of envelope convection and their use for stellar evolution computations. Jørgensen et al. (2018, 2019),
Jørgensen and Weiss (2019), and Mosumgaard et al. (2020) presented detailed procedures to include the mean structure of 3D hydrodynamical simulations as the boundary condition of 1D models to improve their outer stratification. In these studies of patched models, an appropriately calibrated solar model with a structure similar to the underlying 3D simulations is achieved from helioseismology. Houdek et al. (2017) included a full treatment of the interaction between convection and the oscillations.

The measured oscillation frequencies of Kepler targets across stellar evolution are now being used to investigate how the convection-oscillation interaction and the transition between envelope and interior can be achieved from 3D convection simulations for stellar evolution models. Such improvements to stellar structure models partly eliminate the structural contribution to the surface effect, with discrepancies having decreased from about 10 to some $2 \mu \mathrm{Hz}$. Hence the patched models do not yet perform to the level of precision of the asteroseismic data. Moreover, the patching procedures do not deliver reliable post-main-sequence evolution models when performed near the bottom of the convective envelope in currently available 3D simulations. More refined 3D simulations for deeper convective envelopes are needed to improve stellar evolution theory of evolved low-mass stars even further, keeping in mind numerical restrictions; cf. Silva Aguirre et al. (2020).

In addition to global frequency shifts due to surface effects, the p-mode frequencies also undergo time-dependent variability connected with magnetic activity. For the Sun this effect was summarized by Christensen-Dalsgaard (2002). Magnetic effects were found for the solarlike $\mathrm{p}$ modes detected in CoRoT data of the F5V star HD 49933 (García et al., 2010). The Kepler data allowed researchers to study connections between activity and pulsations in samples of Ftype stars (Mathur et al., 2014) and in the legacy sample (Santos et al., 2019), revealing that the p-mode frequency shifts increase with increasing chromospheric activity, increasing metallicity and increasing effective temperature. Young rapid rotators reveal larger frequency shifts than old stars. Moreover, the nonspherical nature of the magnetic activity in the stellar convective envelope changes the frequencies of gravitoacoustic modes (Pérez Hernández et al., 2019). While asteroseismic assessments of the physics of stellar activity in terms of its effect on pulsation-mode behavior has progressed significantly, the improved knowledge is not at a level at which it can be used to encode temporal magnetic activity in the theory of stellar evolution. In this sense, the inclusion of the physics of surface convection via patching of time-averaged 3D stellar atmosphere models to $1 \mathrm{D}$ stellar interiors across the evolution of low-mass stars, via calibrations of solarlike oscillations based on space asteroseismology, advances more steadily and more targeted than the inclusion of magnetic activity.

\section{Improving the theory of angular momentum transport}

A major asset of mixed and g modes is their probing power of the deep stellar interior. While this opportunity does not occur for the Sun and Sun-like dwarfs, we now have thousands of stars with the appropriate $\mathrm{g}$ modes delivering 
their interior rotation rates from measured rotational frequency shifts. Such measurements give quasidirect information about $\Omega_{\text {core }}$ without having to go through Fig. 16. In that sense, the internal rotation of stars has become observational astronomy. Once the Kepler data reached a duration of $2 \mathrm{yr}$, prominent detections of rotational splitting for dipole mixed modes were found in subgiants by Deheuvels et al. (2012, 2014, 2020), and in red giants by Beck et al. (2012), Mosser et al. (2012), and Deheuvels et al. (2015). For intermediate-mass dwarfs, both dipole and quadrupole modes with rotationally split multiplets were detected soon after the nominal 4-yr Kepler light curves became available, with initial exploitations by Kurtz et al. (2014), Pápics et al. (2014, 2015), Saio et al. (2015), and Van Reeth et al. (2015). These studies of internal rotation immediately made it clear that the theory of angular momentum transport as we knew it prior to Kepler failed to explain the asteroseismic data, with discrepancies of up to 2 orders of magnitude in the measured $\Omega_{\text {core }}$.

Many observational derivations of $\Omega_{\text {core }}$ have been done in recent years, confirming the early findings. For red giants these were summarized by Mosser et al. (2014) and Gehan et al. (2018). The discrepancy between theory and observations turned out to be independent of the measured rotation rate during the core-hydrogen burning, i.e., the problem regarding slower than expected near-core rotation is derived for both the perturbative and TAR regimes of rotation. A summary of the asteroseismic results from mixed and $\mathrm{g}$ modes, as well as ways to improve the theory, was offered by Aerts, Mathis, and Rogers (2019) and is not repeated here. One major conclusion of that paper, which summarized data covering all stages of stellar evolution, is that lowand intermediate-mass stars are to a good approximation quasirigid rotators during their core-hydrogen-burning phase, while $\Omega_{\text {core }}$ and $\Omega_{\text {env }}$ values differ by less than a factor of 10 during the RGB phase. The theory of local conservation of angular momentum transport does not explain this. The asteroseismic rotation estimates for 1210 stars across stellar evolution assembled by Aerts, Mathis, and Rogers (2019) reveal that the $\mathrm{CO}$ cores built up inside red giants and subdwarfs by the end of their core-helium-burning phase have the same angular momentum as their white-dwarf successors.

Major updates since the summary by Aerts, Mathis, and Rogers (2019) have become available and are shown in Fig. 6. A large increase in the sample of dwarfs was achieved by $\mathrm{Li}$, Bedding et al. (2019), Li, Van Reeth et al. (2019), and Li et al. (2020), who derived the near-core rotation frequencies for more than 600 F-type g-mode pulsators. These are shown in gray in Fig. 6. Almost all of the newly included F-type dwarfs reveal dipole $(l=1)$ prograde modes, while about $30 \%$ show quadrupole $(l=2)$ modes, and $16 \%$ of them show retrograde Rossby modes. Core rotation rates of 72 core-helium-burning stars have been derived from their dipole mixed modes (Tayar et al., 2019), indicated in blue in Fig. 6. For both of the new samples, there is no asteroseismic estimate of $\log g$, as is the case for all 1210 stars in Fig. 4 given by Aerts, Mathis, and Rogers (2019); hence Fig. 6 was constructed differently. For the red giants addressed by Tayar et al. (2019), $\log g$ was derived from near-IR APOGEE spectroscopy, while Li et al. (2020) relied on $T_{\text {eff }}$ values from Mathur et al. (2017), luminosity estimates from Gaia astrometry computed by
Murphy et al. (2019), and a grid of stellar models to derive the gravity. Although this leads to much larger and more systematic uncertainties for $\log g$ than for the asteroseismic $\log g$ values used by Aerts, Mathis, and Rogers (2019), with uncertainties between 0.2 and 0.5 dex for the stars in Fig. 6 (omitted for clarity), the conclusions by Aerts, Mathis, and Rogers (2019) are fully confirmed by these additional recent studies, representing a tenfold increase in the number of dwarfs with $\Omega_{\text {core }}$.

Following Aerts, Mathis, and Rogers (2019), two major paths have been followed thus far to try to fix the theory of stellar rotation, given the prominent results from asteroseismology. On the one hand, angular momentum transport by IGWs as proposed by Kumar and Quataert (1997) and Rogers et al. (2013, 2015), by mixed modes as studied by Belkacem, Marques, Goupil, Mosser et al. (2015) and Belkacem, Marques, Goupil, Sonoi et al. (2015), and by g modes as in Townsend, Goldstein, and Zweibel (2018) was considered. On the other hand, instabilities due to magnetic fields, termed the magnetic Tayler instability, were considered an explanation by Eggenberger et al. (2019), Fuller, Piro, and Jermyn (2019), and Goldstein, Townsend, and Zweibel (2019). Both physical processes lead to a more efficient evacuation of angular momentum from the core to the envelope of the star than any of the processes that were considered in stellar evolution computations prior to space asteroseismology. While these new theoretical ingredients improve the discrepancies between the asteroseismic measurements of $\Omega_{\text {core }}$ and stellar evolution theory, they still require one to tweak the amount of angular momentum transported from the core to the surface with a free parameter in order to achieve compliance with the measured core rotation rates. In this sense, none of the stellar evolution theories are able to explain the quasirigid rotation measured for stars with ratios of $\Omega / \Omega_{\text {crit }} \in[0,75 \%]$ during the core-hydrogen-burning phase, as displayed in the right panel of Fig. 6.

Van Reeth, Tkachenko, and Aerts (2016), Ouazzani et al. (2017), Christophe et al. (2018), Van Reeth et al. (2018), and Li et al. (2020) measured $\Omega_{\text {core }}$ for a sample of $\sim 650 \gamma$ Dor stars. A dedicated study by Van Reeth et al. (2018) on 37 of those pulsators with high-precision spectroscopy allowed them to assess whether the stars have differential envelope rotation while relying on the theoretical formalism derived by Mathis (2009) as a generalization of the TAR. Van Reeth et al. combined g-mode estimation of $\Omega_{\text {core }}$ with either a p-mode estimation of $\Omega_{\text {env }}$ or a derivation of $\Omega_{\text {surf }}$ from rotational modulation. Li et al. (2020) added 58 more stars to conclude that the rotation is almost rigid to within 5\% for all 95 single F-type dwarfs.

Ongoing modeling work considers two more improvements in addition to the TAR with differential rotation. One generalization takes into account the occurrence of an axisymmetric magnetic field with poloidal and toroidal components following the perturbative approach for the magnetism and was elaborated upon by Prat et al. (2019). The new dispersion relation derived by Prat et al. allows one to assess how such a field affects the g-mode period-spacing pattern. It was found that an interior magnetic field with strength above $10^{5} \mathrm{G}$ leads to pertinent spiky deviations from the tilted 
period-spacing patterns (Van Beeck et al., 2020) that are in principle detectable. These deviating signals have not yet been identified in Kepler data on g-mode pulsators such as those shown in Figs. 11 and 12. Another generalization of the TAR was derived by Mathis and Prat (2019), who computed a new dispersion relation for slightly deformed stars including the centrifugal acceleration. The impact of this inclusion is limited relative to the magnetic effects for rotation rates up to $\sim 70 \% \Omega_{\text {crit }}$ (Henneco et al., 2021).

\section{E. Inference of internal mixing from $g$ modes}

In this section we concentrate on stars of intermediate mass with a radiative envelope. Such stars are much more rapid rotators than low-mass stars with a convective envelope because they do not experience magnetic braking. Models of intermediate-mass stars computed by relying on the Schwarschild or Ledoux criteria of convection, without extra mixing in the near-core region, have convective core masses that are too low. This is deduced from comparing highaccuracy model-independent dynamical masses of doublelined eclipsing binaries to those of stellar evolution models across a wide mass range of $M_{\star} \in[1.2,17] M_{\odot}$ covered by Torres, Andersen, and Giménez (2010), Claret and Torres (2019), and Tkachenko et al. (2020). The need for higher convective core masses for eclipsing binaries stands, irrespective of how $D_{\text {ov }}(r)$ is treated in the isochrone fitting (cf. Fig. 7), as discussed by Constantino and Baraffe (2018), Costa et al. (2019), and Johnston, Tkachenko et al. (2019). Masses of fully mixed convective cores may also be derived from a model-dependent isochrone fitting of the observed extended main-sequence turn-offs (eMSTOs). This was done for numerous young open clusters observed by the Hubble and Gaia space telescopes (Goudfrooij et al., 2018; Li, Sun et al., 2019). Interpretation of the shape and diversity of observed eMSTOs was done mainly by including rotational mixing (Bastian et al., 2018; Gossage et al., 2018) or magnetism (Georgy et al., 2019) in the stellar evolution models. Other causes of mixing, such as the pulsational or tidal wave mixing discussed in Sec. II.A, are usually ignored. Inclusion of $D_{\text {ov }}(r)$ and $D_{\text {mix }}(r)$ profiles calibrated by asteroseismology of single field stars can explain some of the eMSTO properties of young open clusters, thereby impacting their aging. Imposing the asteroseismic results on cluster isochrone fitting allows for higher convective core masses for all stars with $M_{\star} \geq 1.2 M_{\odot}$ (Johnston, Aerts et al., 2019). Cluster aging is a typical area where asteroseismology can be of interest to other fields in astrophysics.

Asteroseismology has provided evidence for the need of higher-than-standard masses of convective cores, in all evolutionary stages and for a large range of stellar masses covering $M_{\star} \in[1.1,25] M_{\odot}$. Backtracking the asteroseismic masses of white dwarfs to earlier evolutionary phases requires more massive helium cores, as discussed by Hermes et al. (2014, 2017a, 2017b). The detailed derivation of the larger-thanexpected inferred $\mathrm{CO}$ core mass of $M_{\mathrm{cc}}=0.45 M_{\odot}$ of the pulsating white dwarf KIC 08626021 (Giammichele et al., 2018) is exemplary of the details that can be derived on the chemical stratification (in this case of oxygen, carbon, and helium) from the exploitation of $g$ modes. The core mass of this white dwarf is about $40 \%$ higher than expected from standard evolution models and points to the need for more $\mathrm{CBM}$ at earlier phases of stellar evolution. The immediate progenitors of the white dwarfs, i.e., the red-giant and subdwarf stars, also reveal the need for CBM and higher core masses than those predicted in stellar evolution theory. This was quantified for three subdwarf B pulsators by Van Grootel et al. (2010), Van Grootel, Charpinet et al. (2010), and Charpinet et al. (2011). These case studies resulted in constraints on the inner $\mathrm{He} / \mathrm{C} / \mathrm{O}$ core from their g modes. Thus, stars not only transport more angular momentum when they have a convective core, they also have CBM resulting in more massive mixed cores than anticipated. This need is most outspoken during the core-hydrogen-burning phase of stellar evolution, so we focus on dwarfs in the rest of this section.

Prior to space asteroseismology, estimation of $D_{\mathrm{ov}}(r)$ assuming convective penetration in Zahn's prescription (Zahn, 1991) led to a wide range of values covering $d_{\text {pen }} \in[0.1,0.5] H_{p}$ for $\beta$ Cep pulsators (Aerts, 2015), but uncertainties from groundbased asteroseismology remained large ( $\gtrsim 0.1)$. At the low-mass end, an extreme case requiring a large overshoot is the F5-type $M_{\star} \simeq 1.5 M_{\odot}$ solarlike p-mode pulsator Procyon. Guenther, Demarque, and Gruberbauer (2014) modeled its p modes from equilibrium models with various prescriptions for $D_{\mathrm{ov}}(r)$, as of the ZAMS, considering a radiative or adiabatic temperature gradient and penetration as well as diffusive overshoot. This led to a fully mixed convective core mass of $M_{\mathrm{cc}} / M_{\star}=12.4 \%$. Space asteroseismology delivered a better estimation of $D_{\text {ov }}(r)$ and $D_{\text {env }}(r)$ from an application of the method in Fig. 16. Most studies had not yet been able to deduce the functional form of the profiles for $D_{\text {ov }}(r)$ and $D_{\text {mix }}(r)$ but had assessed the global level of internal mixing using a forward method, adopting parametrized profiles such as those shown in Fig. 7. The CBM levels and $M_{\mathrm{cc}}$ estimates from Kepler g-mode asteroseismology of a sample of $37 \gamma$ Dor stars for which high-resolution spectroscopy is available revealed equally well explained internal mixing by convective penetration as by diffusive overshooting when using the observational trio $Y^{\text {obs }}=\left(\Pi_{0}, \log T_{\text {eff }}, \log g\right)$ in the modeling via Fig. 16, after estimation of $\Omega_{\text {core }}$, as shown in Fig. 6 (gray circles). The results for the asteroseismic estimation of the stellar parameters $\theta=\left(M_{\star}, \Omega_{\text {core }}, D_{\text {ov }}, \tau, Z_{\text {ini }}\right)$ revealed $M_{\text {cc }} / M_{\star} \in$ $[8,12] \%$ for the sample, which covers the mass range $M_{\star} \in[1.3,1.9] M_{\odot}$, rotation rates $\Omega_{\text {core }} \in[0,25] \mu \mathrm{Hz}$ (i.e., $\Omega_{\text {core }} / \Omega_{\text {crit }} \in[0,70] \%$ ), and the entire core-hydrogen-burning phase; see Fig. 7 given by Mombarg et al. (2019). On the other hand, asteroseismology based on solarlike p modes of nine stars analyzed by Deheuvels et al. (2016) and Hjørringgaard et al. (2017) covering $M_{\star} \in[1.12,1.58] M_{\odot}$ resulted in $M_{\mathrm{cc}} / M_{\star} \in$ $[3,18] \%$, again with equally good results for convective penetration and exponential diffusive overshooting and for models without and with atomic diffusion (the latter without radiative levitation). Angelou et al. (2020) revisited aspects of the methodology to derived $M_{\mathrm{cc}}$ and applied it to 13 stars with solarlike oscillations to arrive at $M_{\mathrm{cc}} / M_{\star} \leq 14 \%$, covering the mass range $M_{\star} \in[0.75,1.45] M_{\odot}$.

Pedersen et al. (2021) fitted measured dipole g-mode period spacings for a sample of 26 Kepler SPB pulsators, using eight grids of stellar models with different CBM and envelope mixing profiles (shown in Fig. 7). This homogeneous 
TABLE I. Inferred convective core masses from estimation of $D_{\mathrm{ov}}(r)$ and $D_{\text {mix }}(r)$ via Fig. 16 for three samples of dwarf pulsators discussed in the text. The level of mixing at the bottom of the envelope, $D_{\text {env }}$, covers a wide range for B stars.

\begin{tabular}{lccccc}
\hline \hline Sample & Spectral type & Mass range & $M_{\text {cc }} / M_{\star}$ range & $\Omega / \Omega_{\text {crit }}$ range & $D_{\text {env }}$ range \\
\hline$\sim 20$ solarlike pulsators & Later than F2 & {$[1.1,1.6] M_{\odot}$} & {$[3,18] \%$} & $<10 \%$ & $?$ \\
$\sim 40$ g-mode pulsators & F0-F2 & {$[1.3,1.9] M_{\odot}$} & {$[7,12] \%$} & {$[0,70] \%$} & $<10 \mathrm{~cm}^{2} \mathrm{~s}^{-1}$ \\
$\sim 30$ g-mode pulsators & B3-B9 & {$[3.3,8.9] M_{\odot}$} & {$[6,29] \%$} & {$[3,96] \%$} & {$\left[12,8.7 \times 10^{5}\right] \mathrm{cm}^{2} \mathrm{~s}^{-1}$} \\
\hline \hline
\end{tabular}

asteroseismic study, via application of the method in Fig. 16 for $\mathcal{P}=8$ and $\mathcal{N}=26$, is the first of its kind for this mass regime, covering stars with a convective core and radiative envelope. It covers the entire main-sequence phase and allowed Pedersen $e t$ $a l$. to infer the overall mixing levels calibrated by the detected dipole $g$ modes for each of the stars and for each of the eight model grids, limiting the solutions for each star to its measured spectroscopic and astrometric values of $T_{\text {eff }}, \log g$, and $\log \left(L / L_{\odot}\right)$. Pedersen et al. (2021) found that 17 of the 26 stars were best modeled via convective penetration and 7 out of 26 were best modeled with exponential diffusive overshooting; see Fig. 7. Moreover, stellar models with a stratified envelope mixing profile (due to vertical shear or IGWs, as graphically depicted in Fig. 7) deliver better asteroseismic fits to the data than unstratified mixing profiles. This study revealed asteroseismic estimates $M_{\mathrm{cc}} / M_{\star} \in[6,29] \%$ for the mass range $M_{\star} \in[3.3,8.9] M_{\odot}$ and rotation rates covering $\Omega_{\text {core }} \in$ $[0.35,21.8] \mu \mathrm{Hz}$ corresponding to $\Omega_{\text {core }} / \Omega_{\text {crit }} \in[3,96] \%$. The level of envelope mixing at the bottom of the radiative envelope where the outer boundary of the $D_{\text {ov }}(r)$ profile occurs (i.e., at the interface of the purple and pink profiles in Fig. 7) reveals a large range for this sample of 26 stars, with values between 12 and $8.7 \times 10^{5} \mathrm{~cm}^{2} \mathrm{~s}^{-1}$. In contrast to the results for F-type g-mode pulsators, this highlights the need for considerable envelope mixing in several of these B-type stars. It is found that the level of mixing at the bottom of the envelope is mildly correlated (correlation coefficient of 0.61) with the rotation frequency in that region. A summary of the asteroseismic inferences of $M_{\mathrm{cc}} / M_{\star}$, via an estimation of $D_{\mathrm{ov}}(r)$ and $D_{\text {env }}(r)$ from imposed profiles as in Fig. 7 , is provided in Table I.

Further improvements in asteroseismic modeling can come from the inclusion of microscopic atomic diffusion in the equilibrium models. Deal et al. $(2017,2018,2020)$ assessed the impact of adding radiative accelerations to model $\mathrm{p}$-mode pulsators with a convective envelope. They compared frequency predictions from 1D models based on atomic diffusion to those from 1D models without diffusion or where it is treated in a simplified way such as by restricting to gravitational settling (of helium or heavier elements). Their studies are based on 1D equilibrium models computed with the CESTAM code (Marques et al., 2013), including an advective and diffusive treatment of rotation. Deal et al. (2020) found that the inclusion of radiative levitation is necessary to achieve reliable values for the p modes of the F-type stars in the Kepler legacy sample with $M_{\star}>1.45 M_{\odot}$, even in the presence of macroscopic rotational mixing. The latter was found to be the dominant element transport process in stars with $M<1.3 M_{\odot}$, while microscopic and macroscopic mixing are of equal importance for the mass range $1.3 M_{\odot}<M<1.45 M_{\odot}$. The importance of radiative levitation for $\mathrm{g}$-mode asteroseismology has been assessed only for two slowly pulsating $\gamma$ Dor stars thus far. This also points to the need to include this process (Mombarg et al., 2020); see also Fig. 5.

The capacity to infer internal mixing profiles, as well as the thermal structure in the CBM region of stars with a convective core, was assessed for dwarfs by Pedersen et al. (2018) and Michielsen et al. (2019) and for core-helium-burning stars by Constantino, Campbell, and Lattanzio (2017). These studies have yet to be put into practice. The full potential of the Kepler data on this front remains underexploited, given that g-mode asteroseismology of dwarfs has become possible only in the past five years, and that such modeling for ensembles of stars is a tedious and time-consuming task following the scheme in Fig. 16. Nevertheless, Table I reveals a large range of envelope mixing in stars of similar mass, metallicity, and evolutionary stage during the main sequence, reflecting the fact that nonlinear interactions between rotation, waves, microscopic atomic diffusion, and magnetism may be at work. Refined calibrations of the mixing due to this multitude of phenomena requires ensemble modeling of g-mode pulsators for hundreds of stars treated in a homogeneous way, instead of the few tens addressed thus far.

\section{F. The beginnings of tidal asteroseismology}

In all of the previous cases, we considered oscillations based upon 1D equilibrium models computed under the assumption of a single star. However, a large fraction of stars occur in binaries, where tidal forces and tidal interactions come into play. The binary fraction among stars increases as the stellar birth mass increases. On average, half of the stars occur in binaries but the occurrence rate for high-mass stars is much higher than for low-mass stars, as high as $\sim 80 \%$ for O-type stars. Their evolution is dominated by binary interactions (Sana et al., 2012).

As long as the orbital separation of the two components or the mass ratio is such that tides can be ignored, the asteroseismic modeling can be done as with single pulsators. The orbital motion may offer stringent and model-independent dynamical masses, particularly for detached double-lined spectroscopic eclipsing binaries. Some wide binaries reveal two pulsating components in the Fourier transform (FT) such that isochrone fitting offers extra constraints compared to the case of a single pulsator. The $\alpha$ Cen system is a prototypical example of this (Miglio and Montalbán, 2005). Other binaries with space photometry covering a variety of pulsating components treated as if concerning a single star were analyzed by Telting et al. (2012), Frandsen et al. (2013), Maxted et al. (2013), Beck et al. (2014), Appourchaux et al. (2015), Baran et al. (2016), Gaulme et al. (2016), Brogaard et al. (2018), Kern et al. (2018), and Themeßl et al. (2018). 
These studies led to stringent constraints on the global stellar parameters thanks to the binarity.

Tidal asteroseismology treats the case of binaries for which the tide-generating potential cannot be ignored in the force balance. For a close binary in which the tidal forces occur in the term $f$ in Eq. (19), the tides come into play at the level of the equilibrium models and for the computation of the oscillations. In such a case, the binarity of a pulsating component implies a complication for an asteroseismic analysis. At the same time, it may offer unique opportunities to test the effects of tidal forces on stellar structure and evolution from tidally excited or tidally affected oscillations. The properties of such oscillations differ from those of selfexcited or stochastically excited oscillations in that they are connected with the orbital frequency, and they therefore offer additional opportunities to probe stellar interiors than modes in single stars. Moreover, tidally excited oscillations may get locked into resonance with the orbit and have a major effect on the evacuation of orbital energy, efficiently changing the binary evolution (Papaloizou and Savonije, 1997; Savonije and Papaloizou, 1997; Witte and Savonije, 1999). Given that the tides come into play and that orbital periods are of the order of days, tidally excited oscillations usually are g modes. However, tidally affected oscillations may also occur among p modes, as found in the close binaries U Gru (Bowman, Johnston et al., 2019) and V453 Cyg (Southworth et al., 2020).

Currently, asteroseismic probing to improve the internal structure from tidal oscillations remains limited. This is not due to a lack of candidate pulsators, as systematic searches for oscillations in eclipsing binaries with CoRoT and Kepler revealed hundreds of cases (Gaulme and Guzik, 2019). Rather, the data analysis to deduce the oscillatory properties is extremely challenging. Iterative schemes have to be devised to unravel the frequencies due to the orbital motion, synchronous, subsynchronous, or supersynchronous rotation, and pulsations. Once again, CoRoT paved the way to the first proper monitoring and iterative orbital and pulsational lightcurve modeling of close binaries with $\mathrm{g}$ modes (Maceroni et al., 2009, 2013) and with p modes (da Silva et al., 2014).

The real breakthrough in the discovery and analysis of tidal oscillations in numerous close binaries came for the 4-yr nominal Kepler light curves. We already showed and discussed the light curve and Fourier transform of the prototype of high-eccentric binaries with tidally excited modes found by Welsh et al. (2011) in Sec. III.A (Fig. 15). That was a case where almost all detected frequencies are exact multiples of the orbital frequency, as expected for dynamical tides. Another situation occurs for the binary KIC 4142768 whose light curve is illustrated in Fig. 19 and discussed by Guo et al. (2019). This is an eclipsing binary with two evolved A-type stars in an eccentric orbit with a period of $14 \mathrm{~d}$. This pulsating binary reveals low-frequency g modes, some but not all of which occur at exact multiples of the orbital frequency (indicated with the red vertical lines in Fig. 19). The binary also undergoes $\kappa$-driven $\delta$ Sct-type p modes in the frequency range $170-220 \mu \mathrm{Hz}$. Spectroscopic follow-up with the HIRES spectrograph at Keck revealed a surface rotation rate only one-fifth that of the pseudosynchronous rate at periastron. The tidally excited modes were identified as quadrupole prograde sectoral modes, as anticipated from the theory of dynamical tides; see Fuller (2017) for a paper on tidally excited oscillations. The frequency range of the detected
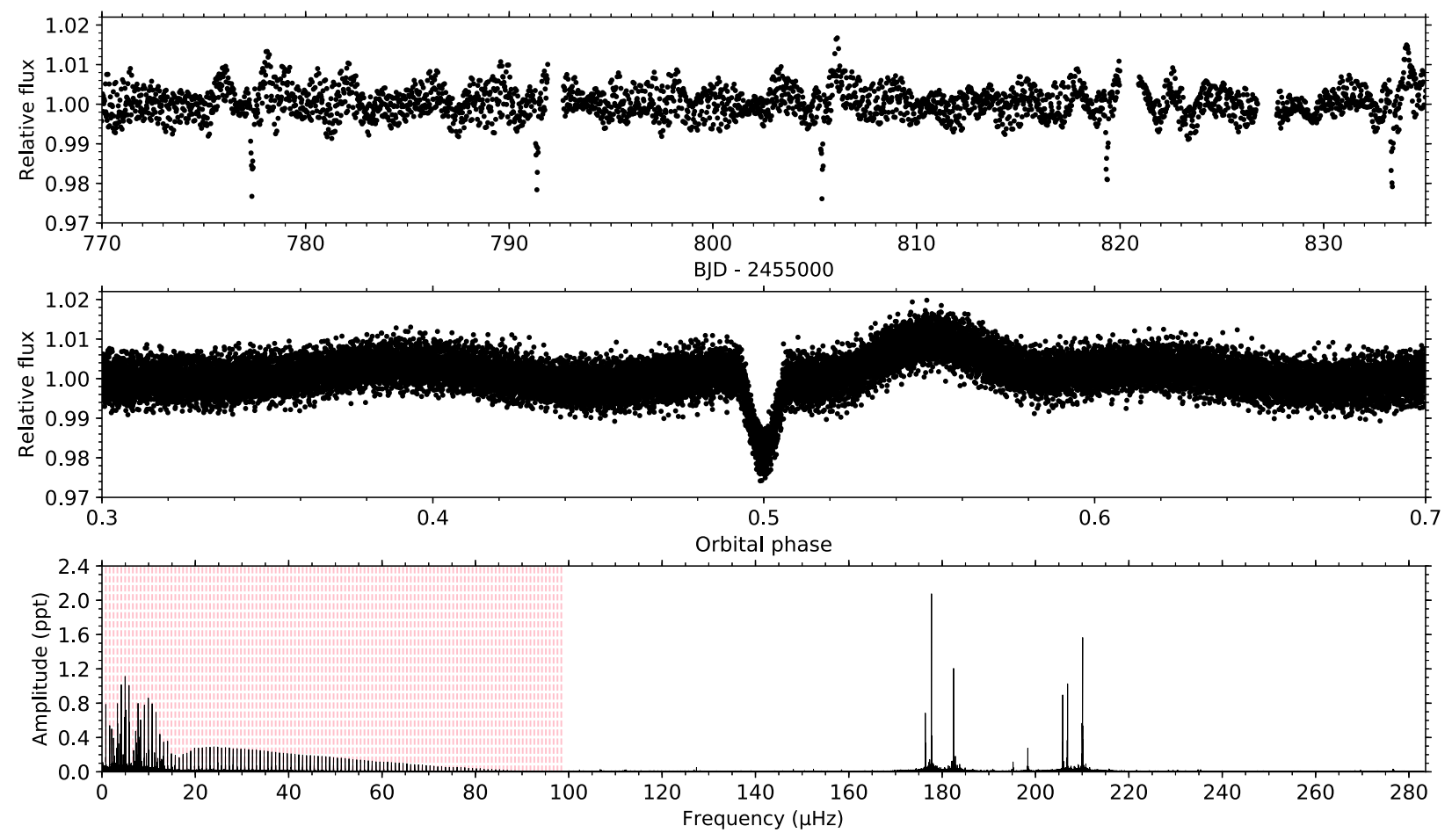

FIG. 19. Excerpt of the Kepler light curve (top panel), phase folded according to an orbital period of $14 \mathrm{~d}$ (middle panel), and the corresponding LS amplitude spectrum (bottom panel) of KIC 4142768. This eclipsing binary reveals self-excited $\kappa$-driven p modes and tidally excited g modes occurring at exact multiples of the orbital frequency (indicated in red). Adapted from Guo et al., 2019. 
self-excited modes is compliant with theoretical predictions for the fundamental parameters of the primary, which is a fairly evolved star close to the terminal-age main sequence. The nearcore rotation rate derived from the fitting of the data to Eq. (49) corresponds to $\Omega_{\text {core }}=0.07 \pm 0.03 \mu \mathrm{Hz}$ and points to an extremely slow rotator, which is in agreement with the spectroscopic surface velocity projected on the line of sight.

Various binaries discovered from Kepler photometry have revealed an oscillation signal at the equilibrium tide, in addition to $\mathrm{g}$ modes triggered by dynamical tides [e.g., KIC 8719324, which was addressed by Thompson et al. (2012)]. Detailed observational analyses were made for several systems similar to KIC 4142768, which is shown in Fig. 19. Examples were given by Debosscher et al. (2013), Hambleton et al. (2013, 2016, 2018), Murphy et al. (2013), Pápics et al. (2013), Borkovits et al. (2014), Fuller et al. (2017), Guo, Gies, and Fuller (2017), Guo, Gies, and Matson (2017), Bowman, Johnston et al. (2019), Guo and Li (2019), Handler et al. (2020), Southworth et al. (2020), and Kurtz et al. (2020), where Handler et al. (2020) and Kurtz et al. (2020) reported the first cases of tip-tilted oblique binary pulsators. Hardly any of these binary systems have yet been modeled asteroseismically, according to Fig. 16, with the exception of the double hybrid $\mathrm{p}$ - and g-mode F-type pulsators KIC 10080943A and KIC 10080943B. Asteroseismic isochrone modeling of the two components revealed the need for extra CBM and higher convective core masses than in standard evolution models for both components, which is in line with Table I (Schmid and Aerts, 2016).

Space photometry continues to deliver a plethora of close binary pulsators, with new discoveries by the day. A large diversity of orbital periods, eccentricities, synchronicities, and oscillation properties have already been found. The extensive paper by Fuller (2017) revisited theories of tidal excitation of nonradial oscillations and provided predictions for flux variations, mode amplitudes, frequencies, phases, and spinorbit misalignment based on a nonadiabatic treatment of the equations, including the Coriolis force. Yet we are only at the beginnings of tidal asteroseismology, because so few systems have been modeled via the scheme in Fig. 16 and we do not yet know how common resonance locking is, nor how important it is in practice for binary evolution and for angular momentum loss. Future modeling work to understand tidal wave transport phenomena in close binaries is in order. Searches for oscillation modes in numerous eclipsing binaries in the TESS data are ongoing. These will undoubtedly uncover objects suitable for better understanding the evolution of massive binaries, including progenitors of future gravitational wave emitters. The TESS sample of high-mass stars observed in its two CVZs holds great potential in this respect.

\section{ROAD MAP FOR THE FUTURE}

The past decade has sparked immense interest in asteroseismology. Following the detection of nonradial oscillation modes in ground-based radial-velocity and/or light curves of a few tens of stars, we have moved on to asteroseismology of tens of thousands of stars covering all evolutionary phases. This success results from uninterrupted long-duration space photometric light curves having ppm-level precision.
Asteroseismology is delivering an observational calibration of internal rotation and mixing across stellar evolution, as a guide for improving the theory of angular momentum and element transport inside stars. The stellar evolution community is currently digesting this flood of asteroseismic information, given the surprises and challenges that it brought. This will eventually lead to better stellar evolution models, as important input for studies of exoplanetary systems and for the chemical evolution of galaxies.

Much more is to come. While past space missions focused on low- and intermediate-mass stars, the current all-sky TESS mission already delivered data for high-mass stars in the Milky Way and LMC during its first two years of operation, covering masses up to $\sim 50 M_{\odot}$. Prospects are excellent that we may embark upon asteroseismology of high-mass binaries on their way to becoming gravitational wave sources, and of blue supergiants nearing their supernova explosion. The methodology in Fig. 16 is in place, but applications should be generalized to a nonadiabatic framework for the oscillations. Moreover, dissection of the maximum amount of information present in the Fourier transforms of the TESS light curves is in order. Asteroseismology based on stochastically excited GIWs looks appealing now that TESS is delivering proper data to guide such a development for high-mass stars. The art will be to distinguish the signatures of coherent gravitoinertial modes and of stochastic GIWs as input for modeling of the stellar interior. Similarly, asteroseismology of pre-main-sequence stars has yet to be put into practice for representative ensembles. Initial studies were based on just a few short light curves (Zwintz et al., 2014), but improvements in the physics of accretion, rotational spin-up, magnetic activity during contraction, and internal mixing of elements as protostars approach their birth are now within reach for asteroseismic scrutiny.

The ongoing NASA TESS and future ESA PLATO missions lift the probing of stellar interiors to all masses and evolutionary stages. With that glorious prospect, asteroseismology is entering the big data era. Machine-learning methods are advantageous for interpreting the massive flux of data but must be applied with proper mathematical modeling, including parameter degeneracies and correlated diagnostics, so as to ascertain appropriate precision estimation of the stellar parameters, among them stellar ages. Ensemble asteroseismology will become ever more powerful when combined with independent and homogeneous nonasteroseismic information coming from all-sky spectroscopic surveys with multiobject spectrographs such as SDSS-V (all-sky, near-IR, time frame 2020-2024) (Kollmeier et al., 2017), WEAVE (Northern Hemisphere, optical, time frame 2020+) (Dalton et al., 2018), and 4MOST (Southern Hemisphere, optical, time frame 2022-2026) (de Jong et al., 2019), along with the final Gaia all-sky space astrometry.

On the theory front, nonlinear asteroseismology has to be redeveloped in this space era. This has major potential given that a high percentage of pulsators reveal departures from linearity and evidence for nonlinear resonant mode coupling. Such coupling occurs in oscillation spectra across all masses and evolutionary stages. Having been found in CoRoT data of $\mathrm{B}$ and Be stars by Degroote et al. (2009) and Huat et al. (2009), it has also been detected in BRITE data of Be stars 
(Baade et al., 2018) and in Kepler photometry of young intermediate-mass stars (Bowman et al., 2016), of subdwarf pulsators (Baran et al., 2012), and in various white dwarfs (Hermes et al., 2015). This observational gold mine is awaiting exploitation in terms of the nonlinear probing of stellar interiors once a modern theoretical framework gets developed, as in Zong, Charpinet, and Vauclair (2016). Similarly, magnetoasteroseismology is still in its infancy. Mode predictions for stellar models with strongly magnetic cores have been triggered to explain mode suppression in red giants by Loi (2020) and Bugnet et al. (2021). Backtracking the results for red giants, their intermediate-mass mainsequence progenitors should also have strong internal magnetic fields. Such fields have a non-negligible effect on $\mathrm{g}$ modes, as shown by the theoretical developments discussed by Prat et al. (2020) and their magnetic signatures predicted by Van Beeck et al. (2020). While these have not yet been found in g-mode period-spacing patterns of Kepler dwarfs, this might be because they have not been looked for with dedicated eyes or with machine-learning artillery.

Finally, we come back to the use of 1D equilibrium models. Several of the stars in Fig. 6 rotate faster than $70 \%$ of their critical rotation frequency. Their asteroseismic modeling will benefit from a 2D treatment. The code ROTORC by Deupree (2001) delivers 2D equilibrium models and was used to make pulsation predictions for $\mathrm{p}$ modes of $\beta$ Cep pulsators (cf. Fig. 1) by Lovekin and Deupree (2008) and Lovekin, Deupree, and Clement (2009). These predictions have not been used in asteroseismic modeling thus far. The public code ESTER (Évolution Stellaire en Rotation) (Rieutord, Espinosa Lara, and Putigny, 2016) is under active development and is advanced in terms of the treatment of transport processes. This code has great potential given the need for improvements in models of the fastest rotating intermediate- and high-mass dwarfs. ESTER delivers 2D axisymmetric static structure models but does not yet treat the chemical evolution of the star, nor 2D mass loss or envelope convection. Proper boundary conditions, including a dynamical wind via 2D nonlocal thermodynamic equilibrium atmosphere models (Petrenz and Puls, 2000), are necessary to improve pulsation predictions for fast rotators. While the current limitations of ESTER can be partially circumvented by fixing the hydrogen mass fraction in the convective core to a seismic estimate of $X_{c}$, as in a recent application to the $\delta$ Sct star Altair (rotating at $\sim 74 \%$ of its critical velocity) (Bouchaud et al., 2020), future developments to turn the code into a full-blown 2D stellar evolution tool would be highly beneficial. This would allow researchers to perform $2 \mathrm{D}$ asteroseismic modeling of the most rapid rotators in Fig. 6, and of the high-mass pulsators discovered by Pedersen et al. (2019), Bowman et al. (2020), Burssens et al. (2020), and Dorn-Wallenstein et al. (2020), in addition to new ones yet to be discovered.

Tremendous progress in our understanding of stellar interiors has been achieved, thanks to nonradial oscillation theory coupled with space photometry of ppm-level precision for thousands of stars. Asteroseismology turned the study of stellar interiors into an observational science. Its future is extremely bright in all aspects of this research field, from instrumentation and ongoing or planned surveys all the way up to fundamental theory. Major improvements for stellar evolution theory based on asteroseismology are under way for single stars, binaries, and star clusters. We thus end with an invitation to those readers whose curiosity might be triggered but who have not yet been active in this field: it is never too late to become an asteroseismologist.

\section{ACKNOWLEDGMENTS}

The writing of this review was initiated while I was lecturing as the 2019 Oort Professor at Leiden Observatory, Netherlands, and at the Max Planck Institute of Astronomy in Heidelberg, Germany. I am grateful for the hospitality at both places, and for the genuine interest in my lectures expressed by young and not-so-young attendees; this made those stays particularly enjoyable. I am also grateful to many colleagues, too numerous to mention, whose inspiring lectures and tutorials educated me in astrophysics in general, and in the computation of numerical stellar models and their oscillation frequencies in particular. Trained as a pure theoretician at master level, I benefited greatly from practical introductions to astronomical observing with various telescopes during my Ph.D. and postdoctorate trajectories. Undertaking the full journey from early instrument concepts to detailed modeling of stellar interiors, in a multicultural and inclusive team spirit, has been crucial for my motivation and inspirational for my supervision and training of the next generations of astrophysicists. The Ph.D. students and postdoctoral researchers in the Leuven asteroseismology team, colleagues from the community, and three referees are thanked for their comments on early versions of the manuscript. I am particularly grateful to my local PYTHON artists in residence, Dominic Bowman, Cole Johnston, Joey Mombarg, Péter Pápics, May Gade Pedersen, and Timothy Van Reeth for producing figures for this review, and to Pieter Degroote for designing the front cover image and animations of nonradial oscillations. Appreciation is also given to Ashley Chontos, Philipp Edelmann, Sylvia Ekström, Gang Li, Tami Rogers, Jamie Tayar, and Kuldeep Verma for providing data or figures in electronic form. Those participants of the conference "Stars and Their Variability Observed from Space," held in Vienna, Austria, in August 2019, who took part in the end-of-conference poll on how our research field should progress, are thanked for their cooperation; the outcome of the poll shaped the final section of this review. I acknowledge funding from the KU Leuven Research Council (Grant No. C16/18/005: PARADISE). The funding received from the European Research Council (ERC) under the European Union's Seventh Framework (FP7/2007-2013/ERC Grant Agreement No. 227224: PROSPERITY, 2009-2013) and Horizon 2020 Research and Innovation Programme (Grant Agreement No. 670519: MAMSIE, 2016-2020) has been essential for the development of my long-term research goals and aspirations. The ERC has allowed me to transform my narrow, bumpy, unconventional path into a broad, royal road.

\section{REFERENCES}

Aerts, C., 2015, in New Windows on Massive Stars, IAU Symposium Vol. 307, edited by G. Meynet, C. Georgy, J. Groh, and P. Stee (Cambridge University Press, Cambridge, England), pp. 154-164, https://doi.org/10.1017/S1743921314006644. 
Aerts, C., J. Christensen-Dalsgaard, and D. W. Kurtz, 2010, Asteroseismology, Astronomy and Astrophysics Library (Springer Science+Business Media, New York), https://doi.org/10.1007/9781-4020-5803-5.

Aerts, C., P. De Cat, R. Kuschnig, J. M. Matthews, D. B. Guenther, A. F. J. Moffat, S. M. Rucinski, D. Sasselov, G. A. H. Walker, and W. W. Weiss, 2006, Astrophys. J. Lett. 642, L165.

Aerts, C., S. Mathis, and T. M. Rogers, 2019, Annu. Rev. Astron. Astrophys. 57, 35.

Aerts, C., and T. M. Rogers, 2015, Astrophys. J. Lett. 806, L33.

Aerts, C., T. Van Reeth, and A. Tkachenko, 2017, Astrophys. J. Lett. 847, L7.

Aerts, C., et al., 2017, Astron. Astrophys. 602, A32.

Aerts, C., et al., 2018a, Mon. Not. R. Astron. Soc. 476, 1234.

Aerts, C., et al., 2018b, Astrophys. J. Suppl. Ser. 237, 15.

Alvan, L., A. S. Brun, and S. Mathis, 2014, Astron. Astrophys. 565, A42.

Alvan, L., A. Strugarek, A. S. Brun, S. Mathis, and R. A. Garcia, 2015, Astron. Astrophys. 581, A112.

An, D., D. M. Terndrup, M. H. Pinsonneault, D. B. Paulson, R. B. Hanson, and J. R. Stauffer, 2007, Astrophys. J. 655, 233.

Anders, F., et al., 2017a, Astron. Astrophys. 600, A70.

Anders, F., et al., 2017b, Astron. Astrophys. 597, A30.

Anderson, R. I., and A. G. Riess, 2018, Astrophys. J. 861, 36.

Angelou, G. C., E. P. Bellinger, S. Hekker, and S. Basu, 2017, Astrophys. J. 839, 116.

Angelou, G. C., E. P. Bellinger, S. Hekker, A. Mints, Y. Elsworth, S. Basu, and A. Weiss, 2020, Mon. Not. R. Astron. Soc. 493, 4987.

Appourchaux, T., 2014, in Asteroseismology, Canary Islands Winter School of Astrophysics Vol. 22, edited by P. L. Pallé and C. Esteban (Cambridge University Press, Cambridge, England), p. 123 [arXiv:1103.5352].

Appourchaux, T., et al., 2008, Astron. Astrophys. 488, 705.

Appourchaux, T., et al., 2012a, Astron. Astrophys. 537, A134.

Appourchaux, T., et al., 2012b, Astron. Astrophys. 543, A54.

Appourchaux, T., et al., 2015, Astron. Astrophys. 582, A25.

Arnett, W. D., C. Meakin, R. Hirschi, A. Cristini, C. Georgy, S. Campbell, L. J. A. Scott, E. A. Kaiser, M. Viallet, and M. Mocák, 2019, Astrophys. J. 882, 18.

Asplund, M., N. Grevesse, A. J. Sauval, and P. Scott, 2009, Annu. Rev. Astron. Astrophys. 47, 481.

Augustson, K. C., and S. Mathis, 2019, Astrophys. J. 874, 83.

Augustson, K. C., S. Mathis, and A. Astoul, 2020, Astrophys. J. 903, 90.

Auvergne, M., P. Bodin, L. Boisnard, J. T. Buey, and S. Chaintreuil, 2009, Astron. Astrophys. 506, 411.

Baade, D., et al., 2016, Astron. Astrophys. 588, A56.

Baade, D., et al., 2018, Astron. Astrophys. 610, A70.

Babusiaux, C., et al. (Gaia Collaboration), 2018, Astron. Astrophys. 616, A10.

Baglin, A., M. Auvergne, P. Barge, M. Deleuil, and E. Michel (CoRoT Exoplanet Science Team), 2009, in Transiting Planets, IAU Symposium Vol. 253, edited by F. Pont, D. Sasselov, and M. J. Holman (Cambridge University Press, Cambridge, England), pp. 71-81, https://doi.org/10.1017/S1743921308026252.

Ball, W. H., B. Beeck, R. H. Cameron, and L. Gizon, 2016, Astron. Astrophys. 592, A159.

Ball, W. H., and L. Gizon, 2014, Astron. Astrophys. 568, A123.

Ball, W. H., and L. Gizon, 2017, Astron. Astrophys. 600, A128.

Ballot, J., F. Lignières, D. R. Reese, and M. Rieutord, 2010, Astron. Astrophys. 518, A30.

Ballot, J., et al., 2011, Astron. Astrophys. 530, A97.
Balona, L. A., 2014, Mon. Not. R. Astron. Soc. 439, 3453.

Baran, A. S., J. H. Telting, P. Németh, R. H. Østensen, M. D. Reed, and F. Kiaeerad, 2016, Astron. Astrophys. 585, A66.

Baran, A. S., et al., 2012, Mon. Not. R. Astron. Soc. 424, 2686.

Barban, C., et al., 2007, Astron. Astrophys. 468, 1033.

Barban, C., et al., 2009, Astron. Astrophys. 506, 51.

Bastian, N., S. Kamann, I. Cabrera-Ziri, C. Georgy, S. Ekström, C. Charbonnel, M. de Juan Ovelar, and C. Usher, 2018, Mon. Not. R. Astron. Soc. 480, 3739.

Bastien, F. A., K. G. Stassun, G. Basri, and J. Pepper, 2013, Nature (London) 500, 427.

Basu, S., and W. J. Chaplin, 2017, Asteroseismic Data Analysis: Foundations and Techniques, Princeton Series in Modern Observational Astronomy (Princeton University Press, Princeton, NJ), https://doi.org/10.1080/00107514.2019.1662096.

Basu, S., and A. Kinnane, 2018, Astrophys. J. 869, 8.

Basu, S., A. Mazumdar, H. M. Antia, and P. Demarque, 2004, Mon. Not. R. Astron. Soc. 350, 277.

Bazot, M., O. Benomar, J. Christensen-Dalsgaard, L. Gizon, S. Hanasoge, M. Nielsen, P. Petit, and K. R. Sreenivasan, 2019, Astron. Astrophys. 623, A125.

Bazot, M., S. Bourguignon, and J. Christensen-Dalsgaard, 2012, Mon. Not. R. Astron. Soc. 427, 1847.

Bazot, M., et al., 2011, Astron. Astrophys. 526, L4.

Beck, P. G., et al., 2011, Science 332, 205.

Beck, P. G., et al., 2012, Nature (London) 481, 55.

Beck, P. G., et al., 2014, Astron. Astrophys. 564, A36.

Bedding, T. R., R. P. Butler, H. Kjeldsen, I. K. Baldry, S. J. O’Toole, C. G. Tinney, G. W. Marcy, F. Kienzle, and F. Carrier, 2001, Astrophys. J. Lett. 549, L105.

Bedding, T. R., S. J. Murphy, I. L. Colman, and D. W. Kurtz, 2015, EPJ Web Conf. 101, 01005.

Bedding, T. R., et al., 2010, Astrophys. J. Lett. 713, L176.

Bedding, T. R., et al., 2011, Nature (London) 471, 608.

Bedding, T. R., et al., 2020, Nature (London) 581, 147.

Belkacem, K., M. A. Dupret, F. Baudin, T. Appourchaux, J. P. Marques, and R. Samadi, 2012, Astron. Astrophys. 540, L7.

Belkacem, K., M. J. Goupil, M. A. Dupret, R. Samadi, F. Baudin, A. Noels, and B. Mosser, 2011, Astron. Astrophys. 530, A142.

Belkacem, K., J. P. Marques, M. J. Goupil, B. Mosser, T. Sonoi, R. M. Ouazzani, M. A. Dupret, S. Mathis, and M. Grosjean, 2015, Astron. Astrophys. 579, A31.

Belkacem, K., J. P. Marques, M. J. Goupil, T. Sonoi, R. M. Ouazzani, M. A. Dupret, S. Mathis, B. Mosser, and M. Grosjean, 2015, Astron. Astrophys. 579, A30.

Belkacem, K., R. Samadi, M. J. Goupil, and M. A. Dupret, 2008, Astron. Astrophys. 478, 163.

Belkacem, K., et al., 2009, Science 324, 1540.

Bell, K. J., J. J. Hermes, A. Bischoff-Kim, S. Moorhead, M. H. Montgomery, R. Østensen, B. G. Castanheira, and D. E. Winget, 2015, Astrophys. J. 809, 14.

Bellinger, E. P., 2019, Mon. Not. R. Astron. Soc. 486, 4612.

Bellinger, E. P., 2020, Mon. Not. R. Astron. Soc. 492, L50.

Bellinger, E. P., G. C. Angelou, S. Hekker, S. Basu, W. H. Ball, and E. Guggenberger, 2016, Astrophys. J. 830, 31.

Bellinger, E. P., S. Basu, S. Hekker, and W. H. Ball, 2017, Astrophys. J. 851, 80.

Benomar, O., et al., 2009, Astron. Astrophys. 507, L13.

Bigot, L., and W. A. Dziembowski, 2002, Astron. Astrophys. 391, 235.

Bildsten, L., G. Ushomirsky, and C. Cutler, 1996, Astrophys. J. 460, 827. 
Bloemen, S., H. Hu, C. Aerts, M. A. Dupret, R. H. Østensen, P. Degroote, E. Müller-Ringat, and T. Rauch, 2014, Astron. Astrophys. 569, A123.

Blomme, R., et al., 2011, Astron. Astrophys. 533, A4.

Borkovits, T., et al., 2014, Mon. Not. R. Astron. Soc. 443, 3068.

Bossini, D., et al., 2017, Mon. Not. R. Astron. Soc. 469, 4718.

Bossini, D., et al., 2019, Astron. Astrophys. 623, A108.

Bouabid, M.-P., M.-A. Dupret, S. Salmon, J. Montalbán, A. Miglio, and A. Noels, 2013, Mon. Not. R. Astron. Soc. 429, 2500.

Bouchaud, K., A. Domiciano de Souza, M. Rieutord, D. R. Reese, and P. Kervella, 2020, Astron. Astrophys. 633, A78.

Bouchy, F., and F. Carrier, 2001, Astron. Astrophys. 374, L5.

Bowman, D. M., 2017, Ph.D. thesis (University of Central Lancashire), https://link.springer.com/book/10.1007\%2F978-3319-66649-5.

Bowman, D. M., S. Burssens, S. Simón-Díaz, P. V. F. Edelmann, T. M. Rogers, L. Horst, F. K. Röpke, and C. Aerts, 2020, Astron. Astrophys. 640, A36.

Bowman, D. M., C. Johnston, A. Tkachenko, D. E. Mkrtichian, K. Gunsriwiwat, and C. Aerts, 2019, Astrophys. J. Lett. 883, L26.

Bowman, D. M., D. W. Kurtz, M. Breger, S. J. Murphy, and D. L. Holdsworth, 2016, Mon. Not. R. Astron. Soc. 460, 1970.

Bowman, D. M., et al., 2019a, Astron. Astrophys. 621, A135.

Bowman, D. M., et al., 2019b, Nat. Astron. 3, 760.

Brassard, P., G. Fontaine, M. Billères, S. Charpinet, J. Liebert, and R. A. Saffer, 2001, Astrophys. J. 563, 1013.

Brassard, P., G. Fontaine, F. Wesemael, and C. J. Hansen, 1992, Astrophys. J. Suppl. Ser. 80, 369.

Breger, M., 2000, Astron. Soc. Pac. Conf. Ser. 210, 3, https://ui .adsabs.harvard.edu/abs/2000ASPC..210....3B.

Breger, M., J. Stich, R. Garrido, B. Martin, S. Y. Jiang, Z. P. Li, D. P. Hube, W. Ostermann, M. Paparo, and M. Scheck, 1993, Astron. Astrophys. 271, 482, http://articles.adsabs.harvard.edu/full/1993A $\% 26$ A...271..482B.

Breger, M., et al., 2005, Astron. Astrophys. 435, 955.

Briquet, M., et al., 2011, Astron. Astrophys. 527, A112.

Brogaard, K., et al., 2018, Mon. Not. R. Astron. Soc. 476, 3729.

Brown, A.G. A., et al. (Gaia Collaboration), 2018, Astron. Astrophys. 616, A1.

Brown, T. M., and R. L. Gilliland, 1994, Annu. Rev. Astron. Astrophys. 32, 37.

Brown, T. M., R. L. Gilliland, R. W. Noyes, and L. W. Ramsey, 1991, Astrophys. J. 368, 599.

Brown, T. M., D. W. Latham, M. E. Everett, and G. A. Esquerdo, 2011, Astron. J. 142, 112.

Buchler, J. R., and M. J. Goupil, 1984, Astrophys. J. 279, 394.

Buchler, J. R., M.-J. Goupil, and C. J. Hansen, 1997, Astron. Astrophys. 321, 159, http://aa.springer.de/papers/7321001/ 2300159/small.htm.

Bugnet, L., V. Prat, S. Mathis, A. Astoul, K. C. Augustson, R. A. Garcia, S. Mathur, L. Amard, and C. Neiner, 2021 (to be published).

Buldgen, G., et al., 2019, Astron. Astrophys. 630, A126.

Burgers, J.M, 1969, Flow Equations for Composite Gases (Academic, New York).

Burssens, S., D. M. Bowman, C. Aerts, M. G. Pedersen, E. Moravveji, and B. Buysschaert, 2019, Mon. Not. R. Astron. Soc. 489, 1304.

Burssens, S., S. Simón-Díaz, D. M. Bowman, G. Holgado, M. Michielsen, A. de Burgos, N. Castro, R. H. Barbá, and C. Aerts, 2020, Astron. Astrophys. 639, A81.

Buysschaert, B., C. Aerts, D. M. Bowman, C. Johnston, T. Van Reeth, M. G. Pedersen, S. Mathis, and C. Neiner, 2018, Astron. Astrophys. 616, A148.
Buysschaert, B., P. G. Beck, E. Corsaro, J. Christensen-Dalsgaard, C. Aerts, T. Arentoft, H. Kjeldsen, R. A. García, V. Silva Aguirre, and P. Degroote, 2016, Astron. Astrophys. 588, A82.

Buzasi, D. L., 2000, in The Third MONS Workshop: Science Preparation and Target Selection, edited by T. Teixeira and T. Bedding (Aarhus University Press, Aarhus, Denmark), p. 9.

Buzasi, D. L., et al., 2005, Astrophys. J. 619, 1072.

Cameron, C., H. Saio, R. Kuschnig, G. A. H. Walker, J. M. Matthews, D. B. Guenther, A. F. J. Moffat, S. M. Rucinski, D. Sasselov, and W. W. Weiss, 2008, Astrophys. J. 685, 489.

Campante, T. L., et al., 2016a, Astrophys. J. 819, 85.

Campante, T. L., et al., 2016b, Astrophys. J. 830, 138.

Cantiello, M., J. Fuller, and L. Bildsten, 2016, Astrophys. J. 824, 14.

Cantiello, M., N. Langer, I. Brott, A. de Koter, S. N. Shore, J. S. Vink, A. Voegler, D. J. Lennon, and S.-C. Yoon, 2009, Astron. Astrophys. 499, 279.

Chaboyer, B., P. Demarque, and M.H. Pinsonneault, 1995, Astrophys. J. 441, 876.

Chaboyer, B., and J. P. Zahn, 1992, Astron. Astrophys. 253, 173, http://articles.adsabs.harvard.edu/full/1992A\%26A...253..173C.

Chaplin, W. J., G. Houdek, C. Karoff, Y. Elsworth, and R. New, 2009, Astron. Astrophys. 500, L21.

Chaplin, W. J., and A. Miglio, 2013, Annu. Rev. Astron. Astrophys. 51, 353.

Chaplin, W. J., et al., 2014, Astrophys. J. Suppl. Ser. 210, 1.

Charbonnel, C., and S. Talon, 2005, Science 309, 2189.

Charpinet, S., P. Brassard, N. Giammichele, and G. Fontaine, 2019, Astron. Astrophys. 628, L2.

Charpinet, S., G. Fontaine, P. Brassard, P. Chayer, F. J. Rogers, C. A. Iglesias, and B. Dorman, 1997, Astrophys. J. Lett. 483, L123.

Charpinet, S., G. Fontaine, P. Brassard, and B. Dorman, 2000, Astrophys. J. Suppl. Ser. 131, 223.

Charpinet, S., et al., 2011, Astron. Astrophys. 530, A3.

Chayer, P., G. Fontaine, and F. Wesemael, 1995, Astrophys. J. Suppl. Ser. 99, 189.

Chiappini, C., et al., 2015, Astron. Astrophys. 576, L12.

Chieffi, A., and M. Limongi, 2013, Astrophys. J. 764, 21.

Choi, J., A. Dotter, C. Conroy, and Y.-S. Ting, 2018, Astrophys. J. 860, 131.

Chontos, A., et al., 2019, Astron. J. 157, 192.

Christensen-Dalsgaard, J., 1984, in Proceedings of the Workshop on Space Research in Stellar Activity and Variability, edited by A. Mangeney and F. Praderie (Observatoire de Paris, Paris), p. 11.

Christensen-Dalsgaard, J., 1988, in Advances in Helio- and Asteroseismology, IAU Symposium Vol. 123, edited by J. ChristensenDalsgaard and S. Frandsen (Springer, New York), p. 295.

Christensen-Dalsgaard, J., 2002, Rev. Mod. Phys. 74, 1073.

Christensen-Dalsgaard, J., C. R. Proffitt, and M. J. Thompson, 1993, Astrophys. J. 403, L75.

Christensen-Dalsgaard, J., and M. J. Thompson, 1993, Astron. Astrophys. 272, L1, http://articles.adsabs.harvard.edu/full/1993A $\% 26$ A...272L...1C.

Christensen-Dalsgaard, J., et al., 2020, Astron. Astrophys. 635, A165.

Christophe, S., J. Ballot, R. M. Ouazzani, V. Antoci, and S. J. A. J. Salmon, 2018, Astron. Astrophys. 618, A47.

Claeskens, G., and N. L. Hjort, 2008, Model Selection and Model Averaging, Cambridge Series in Statistical and Probabilistic Mathematics (Cambridge University Press, Cambridge, England), https://doi.org/10.1017/CBO9780511790485.

Claret, A., and G. Torres, 2019, Astrophys. J. 876, 134. 
Compton, D. L., T. R. Bedding, W. H. Ball, D. Stello, D. Huber, T. R. White, and H. Kjeldsen, 2018, Mon. Not. R. Astron. Soc. 479, 4416.

Constantino, T., and I. Baraffe, 2018, Astron. Astrophys. 618, A177.

Constantino, T., S. W. Campbell, J. Christensen-Dalsgaard, J. C. Lattanzio, and D. Stello, 2015, Mon. Not. R. Astron. Soc. 452, 123.

Constantino, T., S. W. Campbell, and J. C. Lattanzio, 2017, Mon. Not. R. Astron. Soc. 472, 4900.

Corsaro, E., and J. De Ridder, 2014, Astron. Astrophys. 571, A71.

Corsaro, E., J. De Ridder, and R. A. García, 2015, Astron. Astrophys. 579, A83.

Córsico, A. H., L. G. Althaus, M. M. Miller Bertolami, and S. O. Kepler, 2019, Astron. Astrophys. Rev. 27, 7.

Costa, G., L. Girardi, A. Bressan, P. Marigo, T. S. Rodrigues, Y. Chen, A. Lanza, and P. Goudfrooij, 2019, Mon. Not. R. Astron. Soc. 485, 4641.

Cowling, T. G, 1941, Mon. Not. R. Astron. Soc. 101, 367.

Cox, J. P, 1980, Theory of Stellar Pulsation (PSA-2), Princeton Series in Astrophysics (Princeton University Press, Princeton, NJ).

Cox, J.P, and R. T. Giuli, 1968, Principles of Stellar Structure (Gordon and Breach, New York).

Cristini, A., C. Meakin, R. Hirschi, D. Arnett, C. Georgy, and M. Viallet, 2016, Phys. Scr. 91, 034006.

Cunha, M. S., P. P. Avelino, J. Christensen-Dalsgaard, D. Stello, M. Vrard, C. Jiang, and B. Mosser, 2019, Mon. Not. R. Astron. Soc. 490, 909.

Cunha, M. S., D. Stello, P. P. Avelino, J. Christensen-Dalsgaard, and R. H. D. Townsend, 2015, Astrophys. J. 805, 127.

Cunha, M. S., et al., 2007, Astron. Astrophys. Rev. 14, 217.

Cunha, M. S., et al., 2019, Mon. Not. R. Astron. Soc. 487, 3523.

Cuypers, J., et al., 2009, Astron. Astrophys. 499, 967.

Dalton, G., et al., 2018, Proc. SPIE Int. Soc. Opt. Eng. 10702, $107021 \mathrm{~B}$.

da Silva, R., C. Maceroni, D. Gandolfi, H. Lehmann, and A. P. Hatzes, 2014, Astron. Astrophys. 565, A55.

Daszyńska-Daszkiewicz, J., W. A. Dziembowski, A. A. Pamyatnykh, and M.-J. Goupil, 2002, Astron. Astrophys. 392, 151.

Daszyńska-Daszkiewicz, J., A. A. Pamyatnykh, P. Walczak, J. Colgan, C. J. Fontes, and D. P. Kilcrease, 2017, Mon. Not. R. Astron. Soc. 466, 2284.

Davies, G. R., et al., 2015, Mon. Not. R. Astron. Soc. 446, 2959.

Davies, G. R., et al., 2016, Mon. Not. R. Astron. Soc. 456, 2183.

Deal, M., G. Alecian, Y. Lebreton, M. J. Goupil, J. P. Marques, F. LeBlanc, P. Morel, and B. Pichon, 2018, Astron. Astrophys. 618, A10.

Deal, M., M. E. Escobar, S. Vauclair, G. Vauclair, A. Hui-Bon-Hoa, and O. Richard, 2017, Astron. Astrophys. 601, A127.

Deal, M., M. J. Goupil, J. P. Marques, D. R. Reese, and Y. Lebreton, 2020, Astron. Astrophys. 633, A23.

Deal, M., O. Richard, and S. Vauclair, 2016, Astron. Astrophys. 589, A140.

Debosscher, J., L. M. Sarro, C. Aerts, J. Cuypers, B. Vandenbussche, R. Garrido, and E. Solano, 2007, Astron. Astrophys. 475, 1159.

Debosscher, J., et al., 2013, Astron. Astrophys. 556, A56.

De Cat, P., and C. Aerts, 2002, Astron. Astrophys. 393, 965.

Deeming, T. J., 1975, Astrophys. Space Sci. 36, 137.

De Gerónimo, F. C., T. Battich, M. M. Miller Bertolami, L. G. Althaus, and A. H. Córsico, 2019, Astron. Astrophys. 630, A100. Degroote, P., et al., 2009, Astron. Astrophys. 506, 111.

Degroote, P., et al., 2010a, Nature (London) 464, 259.

Degroote, P., et al., 2010b, Astron. Astrophys. 519, A38.
Deheuvels, S., J. Ballot, P. G. Beck, B. Mosser, R. Østensen, R. A. García, and M. J. Goupil, 2015, Astron. Astrophys. 580, A96.

Deheuvels, S., J. Ballot, P. Eggenberger, F. Spada, A. Noll, and J. W. den Hartogh, 2020, Astron. Astrophys. 641, A117.

Deheuvels, S., I. Brandão, V. Silva Aguirre, J. Ballot, E. Michel, M. S. Cunha, Y. Lebreton, and T. Appourchaux, 2016, Astron. Astrophys. 589, A93.

Deheuvels, S., et al., 2010, Astron. Astrophys. 515, A87.

Deheuvels, S., et al., 2012, Astrophys. J. 756, 19.

Deheuvels, S., et al., 2014, Astron. Astrophys. 564, A27.

de Jong, R. S., et al., 2019, The Messenger 175, 3.

Derekas, A., et al., 2011, Science 332, 216.

De Ridder, J., G. Molenberghs, L. Eyer, and C. Aerts, 2016, Astron. Astrophys. 595, L3.

De Ridder, J., et al., 2009, Nature (London) 459, 398.

Deupree, R. G., 2001, Astrophys. J. 552, 268.

Di Mauro, M. P., et al., 2016, Astrophys. J. 817, 65.

Dintrans, B., A. Brandenburg, Å. Nordlund, and R. F. Stein, 2005, Astron. Astrophys. 438, 365.

Domiciano de Souza, A., K. Bouchaud, M. Rieutord, F. Espinosa Lara, and B. Putigny, 2018, Astron. Astrophys. 619, A167.

Domiciano de Souza, A., P. Kervella, S. Jankov, L. Abe, F. Vakili, E. di Folco, and F. Paresce, 2003, Astron. Astrophys. 407, L47.

Domiciano de Souza, A., et al., 2014, Astron. Astrophys. 569, A10.

Dorn-Wallenstein, T.Z., E. M. Levesque, K. F. Neugent, J. R. A. Davenport, B. M. Morris, and K. Gootkin, 2020, Astrophys. J. 902, 24

Dotter, A., C. Conroy, P. Cargile, and M. Asplund, 2017, Astrophys. J. 840, 99.

Dupret, M.-A., A. Grigahcène, R. Garrido, M. Gabriel, and R. Scuflaire, 2005, Astron. Astrophys. 435, 927.

Dupret, M.-A., et al., 2009, Astron. Astrophys. 506, 57.

Dziembowski, W. A., 1971, Acta Astronomica 21, 289, http://articles .adsabs.harvard.edu/full/1971AcA...21..289D.

Dziembowski, W. A., and P. R. Goode, 1992, Astrophys. J. 394, 670.

Dziembowski, W. A., D. O. Gough, G. Houdek, and R. Sienkiewicz, 2001, Mon. Not. R. Astron. Soc. 328, 601.

Dziembowski, W. A., and A. A. Pamyatnykh, 1991, Astron. Astrophys. 248, L11, http://articles.adsabs.harvard.edu/full/ 1991A\%26A...248L..11D.

Eckart, C., 1960, Phys. Fluids 3, 421.

Edelmann, P. V.F., R. P. Ratnasingam, M. G. Pedersen, D. M. Bowman, V. Prat, and T. M. Rogers, 2019, Astrophys. J. 876, 4.

Eggenberger, P., J. W. den Hartogh, G. Buldgen, G. Meynet, S. J. A. J. Salmon, and S. Deheuvels, 2019, Astron. Astrophys. 631, L6.

Eyer, L., et al. (Gaia Collaboration), 2019, Astron. Astrophys. 623, A110.

Fontaine, G., P. Brassard, S. Charpinet, E. M. Green, P. Chayer, M. Billères, and S. K. Rand all, 2003, Astrophys. J. 597, 518.

Foreman-Mackey, D., D. W. Hogg, D. Lang, and J. Goodman, 2013, Publ. Astron. Soc. Pac. 125, 306.

Frandsen, S., et al., 2002, Astron. Astrophys. 394, L5.

Frandsen, S., et al., 2013, Astron. Astrophys. 556, A138.

Freytag, B., H.-G. Ludwig, and M. Steffen, 1996, Astron. Astrophys. 313, 497, http://articles.adsabs.harvard.edu/full/1996A\%26A...313 ..497F.

Fuller, J., 2017, Mon. Not. R. Astron. Soc. 472, 1538.

Fuller, J., M. Cantiello, D. Stello, R. A. Garcia, and L. Bildsten, 2015, Science 350, 423.

Fuller, J., K. Hambleton, A. Shporer, H. Isaacson, and S. Thompson, 2017, Mon. Not. R. Astron. Soc. 472, L25.

Fuller, J., and D. Lai, 2011, Mon. Not. R. Astron. Soc. 412, 1331. 
Fuller, J., and D. Lai, 2012, Mon. Not. R. Astron. Soc. 420, 3126. Fuller, J., D. Lecoanet, M. Cantiello, and B. Brown, 2014, Astrophys. J. 796, 17.

Fuller, J., A. L. Piro, and A. S. Jermyn, 2019, Mon. Not. R. Astron. Soc. 485, 3661.

Gagnier, D., M. Rieutord, C. Charbonnel, B. Putigny, and F. Espinosa Lara, 2019, Astron. Astrophys. 625, A88.

García, R. A., and J. Ballot, 2019, Living Rev. Sol. Phys. 16, 4.

García, R. A., S. Mathur, D. Salabert, J. Ballot, C. Régulo, T. S. Metcalfe, and A. Baglin, 2010, Science 329, 1032.

García, R. A., S. Turck-Chièze, S. J. Jiménez-Reyes, J. Ballot, P. L. Pallé, A. Eff-Darwich, S. Mathur, and J. Provost, 2007, Science 316, 1591.

García, R. A., et al., 2009, Astron. Astrophys. 506, 41.

García, R. A., et al., 2011, Mon. Not. R. Astron. Soc. 414, L6.

García, R. A., et al., 2014, Astron. Astrophys. 568, A10.

Gaulme, P., and J.A. Guzik, 2019, Astron. Astrophys. 630, A106.

Gaulme, P., J. McKeever, J. Jackiewicz, M. L. Rawls, E. Corsaro, B. Mosser, J. Southworth, S. Mahadevan, C. Bender, and R. Deshpande, 2016, Astrophys. J. 832, 121.

Gautschy, A., and H. Saio, 1995, Annu. Rev. Astron. Astrophys. 33, 75.

Gautschy, A., and H. Saio, 1996, Annu. Rev. Astron. Astrophys. 34, 551.

Gehan, C., B. Mosser, E. Michel, R. Samadi, and T. Kallinger, 2018, Astron. Astrophys. 616, A24.

Georgy, C., S. Ekström, A. Granada, G. Meynet, N. Mowlavi, P. Eggenberger, and A. Maeder, 2013, Astron. Astrophys. 553, A24. Georgy, C., et al., 2019, Astron. Astrophys. 622, A66.

Giammichele, N., S. Charpinet, G. Fontaine, P. Brassard, E. M. Green, V. Van Grootel, P. Bergeron, W. Zong, and M.-A. Dupret, 2018, Nature (London) 554, 73.

Gilliland, R. L, et al., 2010, Publ. Astron. Soc. Pac. 122, 131.

Goldreich, P., and Y. Wu, 1999, Astrophys. J. 511, 904.

Goldstein, J., R.H. D. Townsend, and E. G. Zweibel, 2019, Astrophys. J. 881, 66.

Gossage, S., C. Conroy, A. Dotter, J. Choi, P. Rosenfield, P. Cargile, and A. Dolphin, 2018, Astrophys. J. 863, 67.

Goudfrooij, P., L. Girardi, A. Bellini, A. Bressan, M. Correnti, and G. Costa, 2018, Astrophys. J. Lett. 864, L3.

Gough, D., 1985a, Sol. Phys. 100, 65.

Gough, D., 1985b, Nature (London) 314, 14.

Gough, D. O, 1996, Observatory 116, 313, http://articles.adsabs .harvard.edu/full/1996Obs...116..313G.

Gough, D. O, and M. J. Thompson, 1990, Mon. Not. R. Astron. Soc. 242, 25.

Goupil, M.-J., and J. R. Buchler, 1994, Astron. Astrophys. 291, 481, http://articles.adsabs.harvard.edu/full/1994A\%26A...291..481G.

Grassitelli, L., L. Fossati, S. Simón-Diáz, N. Langer, N. Castro, and D. Sanyal, 2015, Astrophys. J. Lett. 808, L31.

Grec, G., E. Fossat, and M. Pomerantz, 1980, Nature (London) 288, 541.

Grosjean, M., M. A. Dupret, K. Belkacem, J. Montalban, R. Samadi, and B. Mosser, 2014, Astron. Astrophys. 572, A11.

Gruber, D., et al., 2012, Mon. Not. R. Astron. Soc. 420, 291.

Gruberbauer, M., D. B. Guenther, and T. Kallinger, 2012, Astrophys. J. 749, 109.

Gruberbauer, M., D. B. Guenther, K. MacLeod, and T. Kallinger, 2013, Mon. Not. R. Astron. Soc. 435, 242.

Guenther, D. B, P. Demarque, and M. Gruberbauer, 2014, Astrophys. J. 787, 164.

Guo, Z., 2020, Astrophys. J. 896, 161.
Guo, Z., J. Fuller, A. Shporer, G. Li, K. Hambleton, J. Manuel, S. Murphy, and H. Isaacson, 2019, Astrophys. J. 885, 46.

Guo, Z., D. R. Gies, and J. Fuller, 2017, Astrophys. J. 834, 59.

Guo, Z., D. R. Gies, and R. A. Matson, 2017, Astrophys. J. 851, 39. Guo, Z., and G. Li, 2019, Astrophys. J. Lett. 882, L5.

Guo, Z., A. Shporer, K. Hambleton, and H. Isaacson, 2020, Astrophys. J. 888, 95.

Guzik, J. A, A. B. Kaye, P. A. Bradley, A. N. Cox, and C. Neuforge, 2000, Astrophys. J. Lett. 542, L57.

Hambleton, K., D. W. Kurtz, A. Prša, S. N. Quinn, J. Fuller, S. J. Murphy, S. E. Thompson, D. W. Latham, and A. Shporer, 2016, Mon. Not. R. Astron. Soc. 463, 1199.

Hambleton, K., et al., 2018, Mon. Not. R. Astron. Soc. 473, 5165.

Hambleton, K. M, D. W. Kurtz, A. Prša, J. A. Guzik, K. Pavlovski, S. Bloemen, J. Southworth, K. Conroy, S. P. Littlefair, and J. Fuller, 2013, Mon. Not. R. Astron. Soc. 434, 925.

Handberg, R., and T. L. Campante, 2011, Astron. Astrophys. 527, A56.

Handler, G., et al., 2006, Mon. Not. R. Astron. Soc. 365, 327.

Handler, G., et al., 2017, Mon. Not. R. Astron. Soc. 464, 2249.

Handler, G., et al., 2019, Astrophys. J. Lett. 873, L4.

Handler, G., et al., 2020, Nat. Astron. 4, 684.

Hansen, C. J, S. D. Kawaler, and V. Trimble, 2004, Stellar Interiors: Physical Principles, Structure, and Evolution, 2nd ed., edited by C. J. Hansen, S. D. Kawaler, and V. Trimble (Springer-Verlag, New York).

Heger, A., N. Langer, and S. E. Woosley, 2000, Astrophys. J. 528, 368.

Hekker, S., and J. Christensen-Dalsgaard, 2017, Astron. Astrophys. Rev. 25, 1.

Hekker, S., Y. Elsworth, and G. C. Angelou, 2018, Astron. Astrophys. 610, A80.

Hekker, S., T. Kallinger, F. Baudin, J. De Ridder, C. Barban, F. Carrier, A. P. Hatzes, W. W. Weiss, and A. Baglin, 2009, Astron. Astrophys. 506, 465.

Hekker, S., et al., 2011, Astron. Astrophys. 525, A131.

Hekker, S., et al., 2012, Astron. Astrophys. 544, A90.

Henneco, J., T. Van Reeth, V. Prat, S. Mathis, J. S. G. Mombarg, and C. Aerts, 2021, arXiv:2101.04116.

Hermes, J. J., M. H. Montgomery, K. J. Bell, P. Chote, B. T. Gänsicke, S. D. Kawaler, J. C. Clemens, B. H. Dunlap, D. E. Winget, and D. J. Armstrong, 2015, Astrophys. J. Lett. 810, L5.

Hermes, J. J., et al., 2014, Astrophys. J. 789, 85.

Hermes, J. J., et al., 2017a, Astrophys. J. Suppl. Ser. 232, 23.

Hermes, J. J., et al., 2017b, Astrophys. J. Lett. 841, L2.

Herwig, F., 2000, Astron. Astrophys. 360, 952, http://ui.adsabs .harvard.edu/abs/2000A\%26A...360..952H/abstract.

Hjørringgaard, J. G., V. Silva Aguirre, T. R. White, D. Huber, B. J. S. Pope, L. Casagrande, A. B. Justesen, and J. Christensen-Dalsgaard, 2017, Mon. Not. R. Astron. Soc. 464, 3713.

Holdsworth, D. L, D. W. Kurtz, B. Smalley, H. Saio, G. Handler, S. J. Murphy, and H. Lehmann, 2016, Mon. Not. R. Astron. Soc. 462, 876.

Hon, M., D. Stello, R. A. García, S. Mathur, S. Sharma, I. L. Colman, and L. Bugnet, 2019, Mon. Not. R. Astron. Soc. 485, 5616.

Horne, J. H, and S. L. Baliunas, 1986, Astrophys. J. 302, 757.

Horst, L., P. V. F. Edelmann, R. Andrássy, F. K. Röpke, D. M. Bowman, C. Aerts, and R.P. Ratnasingam, 2020, Astron. Astrophys. 641, A18.

Houdek, G., N. J. Balmforth, J. Christensen-Dalsgaard, and D. O. Gough, 1999, Astron. Astrophys. 351, 582, http://articles.adsabs .harvard.edu/full/1999A\%26A...351..582H. 
Houdek, G., and M.-A. Dupret, 2015, Living Rev. Sol. Phys. 12, 8. Houdek, G., and D. O. Gough, 2007, Mon. Not. R. Astron. Soc. 375, 861.

Houdek, G., M. N. Lund, R. Trampedach, J. Christensen-Dalsgaard, R. Handberg, and T. Appourchaux, 2019, Mon. Not. R. Astron. Soc. 487, 595.

Houdek, G., R. Trampedach, M. J. Aarslev, and J. ChristensenDalsgaard, 2017, Mon. Not. R. Astron. Soc. 464, L124.

Howell, S. B., et al., 2014, Publ. Astron. Soc. Pac. 126, 398.

Hu, H., C. A. Tout, E. Glebbeek, and M.-A. Dupret, 2011, Mon. Not. R. Astron. Soc. 418, 195.

Huat, A. L., et al., 2009, Astron. Astrophys. 506, 95.

Huber, D., et al., 2008, Astron. Astrophys. 483, 239.

Huber, D., et al., 2012, Astrophys. J. 760, 32.

Huber, D., et al., 2013, Astrophys. J. 767, 127.

Huber, D., et al., 2017, Astrophys. J. 844, 102.

Jeffery, C. S, and H. Saio, 2006, Mon. Not. R. Astron. Soc. 372, L48.

Jiang, C., J. Christensen-Dalsgaard, and M. Cunha, 2018, Mon. Not. R. Astron. Soc. 474, 5413.

Johnson, R. A, and D. W. Wichern, 2007, Applied Multivariate Statistical Analysis, 6th ed. (Pearson Prentice Hall, Englewood Cliffs, NJ).

Johnston, C., C. Aerts, M. G. Pedersen, and N. Bastian, 2019, Astron. Astrophys. 632, A74.

Johnston, C., A. Tkachenko, C. Aerts, G. Molenberghs, D. M. Bowman, M. G. Pedersen, B. Buysschaert, and P. I. Pápics, 2019, Mon. Not. R. Astron. Soc. 482, 1231.

Jørgensen, A. C. S., J. R. Mosumgaard, A. Weiss, V. Silva Aguirre, and J. Christensen-Dalsgaard, 2018, Mon. Not. R. Astron. Soc. 481, L35.

Jørgensen, A. C. S., and A. Weiss, 2019, Mon. Not. R. Astron. Soc. 488, 3463.

Jørgensen, A.C.S., A. Weiss, G. Angelou, and V. Silva Aguirre, 2019, Mon. Not. R. Astron. Soc. 484, 5551.

Joyce, M., and B. Chaboyer, 2018, Astrophys. J. 856, 10.

Kallinger, T., J. De Ridder, S. Hekker, S. Mathur, B. Mosser, M. Gruberbauer, R. A. García, C. Karoff, and J. Ballot, 2014, Astron. Astrophys. 570, A41.

Kallinger, T., et al., 2010, Astron. Astrophys. 509, A77.

Kallinger, T., et al., 2017, Astron. Astrophys. 603, A13.

Karoff, C., T. L. Campante, J. Ballot, T. Kallinger, M. Gruberbauer, R. A. García, D. A. Caldwell, J. L. Christiansen, and K. Kinemuchi, 2013, Astrophys. J. 767, 34.

Keen, M. A., T. R. Bedding, S. J. Murphy, V. S. Schmid, C. Aerts, A. Tkachenko, R. M. Ouazzani, and D. W. Kurtz, 2015, Mon. Not. R. Astron. Soc. 454, 1792.

Kern, J. W., M.D. Reed, A. S. Baran, J.H. Telting, and R. H. Østensen, 2018, Mon. Not. R. Astron. Soc. 474, 4709.

Kervella, P., and A. Domiciano de Souza, 2006, Astron. Astrophys. 453, 1059.

Kilkenny, D., C. Koen, D. O’Donoghue, and R. S. Stobie, 1997, Mon. Not. R. Astron. Soc. 285, 640.

Kilkenny, D., C. Koen, D. O'Donoghue, F. van Wyk, K. A. Larson, R. Shobbrook, D. J. Sullivan, M. R. Burleigh, P. D. Dobbie, and S. D. Kawaler, 1999, Mon. Not. R. Astron. Soc. 303, 525.

Kippenhahn, R., A. Weigert, and A. Weiss, 2012, Stellar Structure and Evolution, Astronomy and Astrophysics Library (SpringerVerlag, Berlin).

Kjeldsen, H., and T. R. Bedding, 1995, Astron. Astrophys. 293, 87, http://articles.adsabs.harvard.edu/full/1995A\%26A...293...87K.

Kjeldsen, H., T. R. Bedding, and J. Christensen-Dalsgaard, 2008, Astrophys. J. Lett. 683, L175.
Kjeldsen, H., T. R. Bedding, M. Viskum, and S. Frandsen, 1995, Astron. J. 109, 1313.

Koch, D. G., et al., 2010, Astrophys. J. Lett. 713, L79.

Kollmeier, J. A., et al., 2017, arXiv:1711.03234.

Krtička, J., and A. Feldmeier, 2018, Astron. Astrophys. 617, A121.

Kumar, P., and E. J. Quataert, 1997, Astrophys. J. Lett. 475, L143.

Kurtz, D. W., 1985, Mon. Not. R. Astron. Soc. 213, 773.

Kurtz, D. W., 1990, Annu. Rev. Astron. Astrophys. 28, 607.

Kurtz, D. W., H. Saio, M. Takata, H. Shibahashi, S. J. Murphy, and T. Sekii, 2014, Mon. Not. R. Astron. Soc. 444, 102.

Kurtz, D. W., H. Shibahashi, S. J. Murphy, T. R. Bedding, and D. M. Bowman, 2015, Mon. Not. R. Astron. Soc. 450, 3015.

Kurtz, D. W., et al., 2005, Mon. Not. R. Astron. Soc. 358, 651.

Kurtz, D. W., et al., 2011, Mon. Not. R. Astron. Soc. 414, 2550.

Kurtz, D. W., et al., 2020, Mon. Not. R. Astron. Soc. 494, 5118.

Laplace, P. S., 1799, Traité de Mécanique Céleste (Imprimerie de Crapelet, Paris).

Lebreton, Y., and M. J. Goupil, 2014, Astron. Astrophys. 569, A21.

Lebreton, Y., M. J. Goupil, and J. Montalbán, 2014, EAS Publ. Ser., 65, 177.

Ledoux, P., 1951, Astrophys. J. 114, 373.

Ledoux, P., 1962, Bull. Cl. Sci., Acad. R. Belg. 48, 240.

Ledoux, P., and T. Walraven, 1958, Stellar Stability, Handbuch der Physik Vol. 51, p. 353, https://doi.org/10.1007/978-3-642-459085_6.

Lee, U., and I. Baraffe, 1995, Astron. Astrophys. 301, 419, http:// articles.adsabs.harvard.edu/full/1995A\%26A...301..419L.

Lee, U., and H. Saio, 1987a, Mon. Not. R. Astron. Soc. 224, 513.

Lee, U., and H. Saio, 1987b, Mon. Not. R. Astron. Soc. 225, 643.

Lee, U., and H. Saio, 1989, Mon. Not. R. Astron. Soc. 237, 875.

Lee, U., and H. Saio, 1997, Astrophys. J. 491, 839.

Lee, U., and H. Saio, 2020, Mon. Not. R. Astron. Soc. 497, 4117.

Lefever, K., J. Puls, and C. Aerts, 2007, Astron. Astrophys. 463, 1093.

Li, C., W. Sun, R. de Grijs, L. Deng, K. Wang, G. Cordoni, and A. P. Milone, 2019, Astrophys. J. 876, 65.

Li, G., T. R. Bedding, S. J. Murphy, T. Van Reeth, V. Antoci, and R.-M. Ouazzani, 2019, Mon. Not. R. Astron. Soc. 482, 1757.

Li, G., T. Van Reeth, T. R. Bedding, S. J. Murphy, and V. Antoci, 2019, Mon. Not. R. Astron. Soc. 487, 782.

Li, G., T. Van Reeth, T. R. Bedding, S. J. Murphy, V. Antoci, R.-M. Ouazzani, and N. H. Barbara, 2020, Mon. Not. R. Astron. Soc. 491, 3586.

Lignières, F., M. Rieutord, and D. Reese, 2006, Astron. Astrophys. 455, 607 .

Loi, S. T., 2020, Mon. Not. R. Astron. Soc. 496, 3829.

Loi, S. T, and J. C. B. Papaloizou, 2020, Mon. Not. R. Astron. Soc. 491, 708.

Longair, M., 2001, Publ. Astron. Soc. Pac. 113, 1.

Loumos, G. L., and T. J. Deeming, 1978, Astrophys. Space Sci. 56, 285.

Lovekin, C. C., and R. G. Deupree, 2008, Astrophys. J. 679, 1499.

Lovekin, C. C., R. G. Deupree, and M. J. Clement, 2009, Astrophys. J. 693, 677.

Lund, M. N., et al., 2017, Astrophys. J. 835, 172.

Lundkvist, M. S., et al., 2016, Nat. Commun. 7, 11201.

Maceroni, C., J. Montalbán, D. Gandolfi, K. Pavlovski, and M. Rainer, 2013, Astron. Astrophys. 552, A60.

Maceroni, C., et al., 2009, Astron. Astrophys. 508, 1375.

Maeder, A., 1999, Astron. Astrophys. 347, 185, http://articles.adsabs .harvard.edu/full/1999A\%26A...347..185M.

Maeder, A., 2003, Astron. Astrophys. 399, 263. 
Maeder, A., 2009, Physics, Formation and Evolution of Rotating Stars, Astronomy and Astrophysics Library (Springer, Berlin), https://doi.org/10.1007/978-3-540-76949-1.

Magic, Z., and A. Weiss, 2016, Astron. Astrophys. 592, A24.

Maia, M. T., J. Meléndez, D. Lorenzo-Oliveira, L. Spina, and P. Jofré, 2019, Astron. Astrophys. 628, A126.

Marques, J. P., et al., 2013, Astron. Astrophys. 549, A74.

Martins, F., and A. Palacios, 2013, Astron. Astrophys. 560, A16.

Mathias, P., et al., 2004, Astron. Astrophys. 417, 189.

Mathis, S., 2009, Astron. Astrophys. 506, 811.

Mathis, S., 2013, in Studying Stellar Rotation and Convection: Theoretical Background and Seismic Diagnostics, Lecture Notes in Physics Vol. 865, edited by M. Goupil, K. Belkacem, C. Neiner, F. Lignières, and J. J. Green (Springer-Verlag, Berlin), p. 23.

Mathis, S., and V. Prat, 2019, Astron. Astrophys. 631, A26.

Mathur, S., R. A. García, J. Ballot, T. Ceillier, D. Salabert, T. S. Metcalfe, C. Régulo, A. Jiménez, and S. Bloemen, 2014, Astron. Astrophys. 562, A124.

Mathur, S., R. A. García, L. Bugnet, Â. R. G. Santos, N. Santiago, and P. G. Beck, 2019, Front. Astron. Space Sci. 6, 46.

Mathur, S., R. A. García, D. Huber, C. Regulo, D. Stello, P. G. Beck, K. Houmani, and D. Salabert, 2016, Astrophys. J. 827, 50.

Mathur, S., et al., 2010, Astron. Astrophys. 518, A53.

Mathur, S., et al., 2011, Astrophys. J. 741, 119.

Mathur, S., et al., 2017, Astrophys. J. Suppl. Ser. 229, 30.

Matthews, J. M., 2007, Commun. Asteroseismol. 150, 333.

Maxted, P. F. L., et al., 2013, Nature (London) 498, 463.

Mazumdar, A., et al., 2014, Astrophys. J. 782, 18.

McKeever, J. M., S. Basu, and E. Corsaro, 2019, Astrophys. J. 874, 180.

Meakin, C. A., and D. Arnett, 2007, Astrophys. J. 667, 448.

Meibom, S., S. A. Barnes, I. Platais, R. L. Gilliland, D. W. Latham, and R. D. Mathieu, 2015, Nature (London) 517, 589.

Metcalfe, T. S., et al., 2014, Astrophys. J. Suppl. Ser. 214, 27.

Michaud, G., G. Alecian, and J. Richer, 2015, Atomic Diffusion in Stars, Astronomy and Astrophysics Library (Springer International Publishing Cham, Switzerland).

Michaud, G., O. Richard, J. Richer, and D. A. VandenBerg, 2004, Astrophys. J. 606, 452.

Michel, E., et al., 2008, Science 322, 558.

Michielsen, M., M. G. Pedersen, K. C. Augustson, S. Mathis, and C. Aerts, 2019, Astron. Astrophys. 628, A76.

Miglio, A., and J. Montalbán, 2005, Astron. Astrophys. 441, 615.

Miglio, A., J. Montalbán, F. Baudin, P. Eggenberger, A. Noels, S.

Hekker, J. De Ridder, W. Weiss, and A. Baglin, 2009, Astron. Astrophys. 503, L21.

Miglio, A., J. Montalbán, A. Noels, and P. Eggenberger, 2008, Mon. Not. R. Astron. Soc. 386, 1487.

Miglio, A., et al., 2010, Astron. Astrophys. 520, L6.

Miglio, A., et al., 2013, Mon. Not. R. Astron. Soc. 429, 423.

Mombarg, J. S. G., A. Dotter, T. Van Reeth, A. Tkachenko, S. Gebruers, and C. Aerts, 2020, Astrophys. J. 895, 51.

Mombarg, J. S. G., T. Van Reeth, M. G. Pedersen, G. Molenberghs, D. M. Bowman, C. Johnston, A. Tkachenko, and C. Aerts, 2019, Mon. Not. R. Astron. Soc. 485, 3248.

Monteiro, M. J. P. F. G., 2009, Ed., Evolution and Seismic Tools for Stellar Astrophysics, Vol. 316 (Springer, New York).

Monteiro, M. J.P. F. G., J. Christensen-Dalsgaard, and M. J. Thompson, 1994, Astron. Astrophys. 283, 247, http://articles .adsabs.harvard.edu/full/1994A\%26A...283..247M.

Monteiro, M. J. P. F. G., J. Christensen-Dalsgaard, and M. J. Thompson, 2000, Mon. Not. R. Astron. Soc. 316, 165.
Monteiro, M. J. P. F. G., and M. J. Thompson, 2005, Mon. Not. R. Astron. Soc. 361, 1187.

Montgomery, M. H., 2005, Astrophys. J. 633, 1142.

Montgomery, M. H., J. J. Hermes, D. E. Winget, B. H. Dunlap, and K. J. Bell, 2020, Astrophys. J. 890, 11.

Montgomery, M. H., and D. O’Donoghue, 1999, Delta Scuti Star Newsl. 13, 28, http://articles.adsabs.harvard.edu/full/1999DSSN.. .13...28M.

Moravveji, E., 2016, Mon. Not. R. Astron. Soc. 455, L67.

Moravveji, E., C. Aerts, P. I. Pápics, S. A. Triana, and B. Vandoren, 2015, Astron. Astrophys. 580, A27.

Moravveji, E., A. Moya, and E. F. Guinan, 2012, Astrophys. J. 749, 74.

Moravveji, E., R. H. D. Townsend, C. Aerts, and S. Mathis, 2016, Astrophys. J. 823, 130.

Moskalik, P., and W. A. Dziembowski, 1992, Astron. Astrophys. 256, L5, http://articles.adsabs.harvard.edu/full/1992A\%26A.. $.256 \mathrm{~L} \ldots 5 \mathrm{M}$.

Mosser, B., and T. Appourchaux, 2009, Astron. Astrophys. 508, 877.

Mosser, B., K. Belkacem, C. Pinçon, M. Takata, M. Vrard, C. Barban, M.-J. Goupil, T. Kallinger, and R. Samadi, 2017, Astron. Astrophys. 598, A62.

Mosser, B., C. Gehan, K. Belkacem, R. Samadi, E. Michel, and M. J. Goupil, 2018, Astron. Astrophys. 618, A109.

Mosser, B., C. Pinçon, K. Belkacem, M. Takata, and M. Vrard, 2017, Astron. Astrophys. 600, A1.

Mosser, B., M. Vrard, K. Belkacem, S. Deheuvels, and M. J. Goupil, 2015, Astron. Astrophys. 584, A50.

Mosser, B., et al., 2010, Astron. Astrophys. 517, A22.

Mosser, B., et al., 2012, Astron. Astrophys. 548, A10.

Mosser, B., et al., 2013, Astron. Astrophys. 559, A137.

Mosser, B., et al., 2014, Astron. Astrophys. 572, L5.

Mosumgaard, J. R, A. C. Sølvsten Jørgensen, A. Weiss, V. Silva Aguirre, and J. Christensen-Dalsgaard, 2020, Mon. Not. R. Astron. Soc., 491, 1160.

Murphy, S. J., D. Hey, T. Van Reeth, and T. R. Bedding, 2019, Mon. Not. R. Astron. Soc. 485, 2380.

Murphy, S. J., H. Shibahashi, and D. W. Kurtz, 2013, Mon. Not. R. Astron. Soc. 430, 2986.

Murphy, S. J., et al., 2013, Mon. Not. R. Astron. Soc. 432, 2284.

Neiner, C., U. Lee, S. Mathis, H. Saio, C. C. Lovekin, and K. C. Augustson, 2020, Astron. Astrophys., 644, A9.

Neiner, C., et al., 2012, Astron. Astrophys. 546, A47.

North, J. R., J. Davis, T. R. Bedding, M. J. Ireland, A. P. Jacob, J. O’Byrne, S. M. Owens, J. G. Robertson, W. J. Tango, and P. G. Tuthill, 2007, Mon. Not. R. Astron. Soc. 380, L80.

Østensen, R. H., J. H. Telting, M. D. Reed, A. S. Baran, P. Nemeth, and F. Kiaeerad, 2014, Astron. Astrophys. 569, A15.

Ouazzani, R.-M., M.-A. Dupret, and D. R. Reese, 2012, Astron. Astrophys. 547, A75.

Ouazzani, R.-M., S. J. A. J. Salmon, V. Antoci, T. R. Bedding, S. J. Murphy, and I. W. Roxburgh, 2017, Mon. Not. R. Astron. Soc. 465, 2294.

Ouazzani, R. M., F. Lignières, M. A. Dupret, S. J. A. J. Salmon, J. Ballot, S. Christophe, and M. Takata, 2020, Astron. Astrophys. 640, A49.

Ouazzani, R. M., J. P. Marques, M. J. Goupil, S. Christophe, V. Antoci, S. J. A. J. Salmon, and J. Ballot, 2019, Astron. Astrophys. 626, A121.

Pablo, H., et al., 2016, Publ. Astron. Soc. Pac. 128, 125001.

Pamyatnykh, A. A, 1999, Acta Astron. 49, 119, http://acta.astrouw .edu.p1/Vol49/n2/a_49_2_1.html.

Papaloizou, J., and J. E. Pringle, 1978, Mon. Not. R. Astron. Soc. 182, 423. 
Papaloizou, J. C. B., and G. J. Savonije, 1997, Mon. Not. R. Astron. Soc. 291, 651.

Pápics, P. I., 2012, Astron. Nachr. 333, 1053.

Pápics, P. I, 2013, Ph.D. thesis (KU Leuven), https://ui.adsabs .harvard.edu/abs/2013PhDT........6P/abstract.

Pápics, P. I., E. Moravveji, C. Aerts, A. Tkachenko, S. A. Triana, S. Bloemen, and J. Southworth, 2014, Astron. Astrophys. 570, A8.

Pápics, P. I., A. Tkachenko, C. Aerts, T. Van Reeth, K. De Smedt, M. Hillen, R. Østensen, and E. Moravveji, 2015, Astrophys. J. Lett. 803, L25.

Pápics, P. I., et al., 2013, Astron. Astrophys. 553, A127.

Pápics, P. I., et al., 2017, Astron. Astrophys. 598, A74.

Paquette, C., C. Pelletier, G. Fontaine, and G. Michaud, 1986, Astrophys. J. Suppl. Ser. 61, 177.

Paxton, B., L. Bildsten, A. Dotter, F. Herwig, P. Lesaffre, and F. Timmes, 2011, Astrophys. J. Suppl. Ser. 192, 3.

Paxton, B., et al., 2013, Astrophys. J. Suppl. Ser. 208, 4.

Paxton, B., et al., 2015, Astrophys. J. Suppl. Ser. 220, 15.

Paxton, B., et al., 2018, Astrophys. J. Suppl. Ser. 234, 34.

Paxton, B., et al., 2019, Astrophys. J. Suppl. Ser. 243, 10.

Pedersen, M. G., C. Aerts, P. I. Pápics, M. Michielsen, S. Gebruers, T. M. Rogers, G. Molenberghs, S. Burssens, S. R. G. Castaneda, and D. M. Bowman, 2021 (to be published).

Pedersen, M. G., C. Aerts, P. I. Pápics, and T. M. Rogers, 2018, Astron. Astrophys. 614, A128.

Pedersen, M. G., et al., 2019, Astrophys. J. Lett. 872, L9.

Pérez Hernández, F., R. A. García, S. Mathur, A. R. G. Santos, and C. Régulo, 2019, Front. Astron. Space Sci. 6, 41.

Petrenz, P., and J. Puls, 2000, Astron. Astrophys. 358, 956, http:// articles.adsabs.harvard.edu/full/2000A\%26A...358..956P.

Pigulski, A., et al., 2016, Astron. Astrophys. 588, A55.

Pinçon, C., M. J. Goupil, and K. Belkacem, 2019, Astron. Astrophys., arXiv:1912.06008.

Pinsonneault, M., 1997, Annu. Rev. Astron. Astrophys. 35, 557.

Pinsonneault, M. H., et al., 2018, Astrophys. J. Suppl. Ser. 239, 32.

Pope, B. J. S., et al., 2019, Astrophys. J. Suppl. Ser. 245, 8.

Prat, V., S. Mathis, K. Augustson, F. Lignières, J. Ballot, L. Alvan, and A. S. Brun, 2018, Astron. Astrophys. 615, A106.

Prat, V., S. Mathis, B. Buysschaert, J. Van Beeck, D. M. Bowman, C. Aerts, and C. Neiner, 2019, Astron. Astrophys. 627, A64.

Prat, V., S. Mathis, F. Lignières, J. Ballot, and P.-M. Culpin, 2017, Astron. Astrophys. 598, A105.

Prat, V., S. Mathis, C. Neiner, J. Van Beeck, D. M. Bowman, and C. Aerts, 2020, Astron. Astrophys. 636, A100.

Przybilla, N., M. F. Nieva, A. Irrgang, and K. Butler, 2013, EAS Publ. Ser. 63, 13.

Ramiaramanantsoa, T., et al., 2018, Mon. Not. R. Astron. Soc. 473, 5532.

Ratnasingam, R. P, P. V. F. Edelmann, and T. M. Rogers, 2019, Mon. Not. R. Astron. Soc. 482, 5500.

Rauer, H., et al., 2014, Exp. Astron. 38, 249.

Reed, M. D., H. Foster, J. H. Telting, R. H. Østensen, L. H. Farris, R. Oreiro, and A. S. Baran, 2014, Mon. Not. R. Astron. Soc. 440, 3809.

Reed, M. D., et al., 2011, Mon. Not. R. Astron. Soc. 414, 2885.

Reese, D., F. Lignières, and M. Rieutord, 2006, Astron. Astrophys. 455, 621.

Reese, D. R., V. Prat, C. Barban, C. van 't Veer-Menneret, and K. B. MacGregor, 2013, Astron. Astrophys. 550, A77.

Rendle, B. M., et al., 2019, Mon. Not. R. Astron. Soc. 490, 4465.

Richard, O., G. Michaud, and J. Richer, 2002, Astrophys. J. 580, 1100.

Richard, O., G. Michaud, J. Richer, S. Turcotte, S. Turck-Chièze, and D. A. VandenBerg, 2002, Astrophys. J. 568, 979.
Richer, J., G. Michaud, and S. Turcotte, 2000, Astrophys. J. 529, 338.

Ricker, G. R., et al., 2016, Proc. SPIE Int. Soc. Opt. Eng. 9904, 99042B.

Rieutord, M., F. Espinosa Lara, and B. Putigny, 2016, J. Comput. Phys. 318, 277.

Rogers, T., and G. Glatzmaier, 2005, Mon. Not. R. Astron. Soc. 364, 1135.

Rogers, T. M., 2015, Astrophys. J. Lett. 815, L30.

Rogers, T. M., D. N.C. Lin, J. N. McElwaine, and H.H.B. Lau, 2013, Astrophys. J. 772, 21.

Rogers, T. M., and J. N. McElwaine, 2017, Astrophys. J. Lett. 848, L1.

Romero, A. D., et al., 2017, Astrophys. J. 851, 60.

Rosenthal, C. S., J. Christensen-Dalsgaard, Å. Nordlund, R. F. Stein, and R. Trampedach, 1999, Astron. Astrophys. 351, 689, http:// articles.adsabs.harvard.edu/full/1999A\%26A...351..689R.

Rowe, J. F., et al., 2006, Commun. Asteroseismol. 148, 34.

Roxburgh, I. W., 2005, Astron. Astrophys. 434, 665.

Roxburgh, I. W., and S. V. Vorontsov, 2003, Astron. Astrophys. 411, 215.

Roxburgh, I. W., and S. V. Vorontsov, 2006, Mon. Not. R. Astron. Soc. 369, 1491.

Sahlholdt, C. L., and V. Silva Aguirre, 2018, Mon. Not. R. Astron. Soc. 481, L125.

Saio, H., 1981, Astrophys. J. 244, 299.

Saio, H., 1982, Astrophys. J. 256, 717.

Saio, H., 2014, in Precision Asteroseismology, IAU Symposium Vol. 301, edited by J. A. Guzik, W. J. Chaplin, G. Handler, and A. Pigulski (Cambridge University Press, Cambridge, England), pp. 197-204, https://doi.org/10.1017/S1743921313014324.

Saio, H., T. R. Bedding, D. W. Kurtz, S. J. Murphy, V. Antoci, H. Shibahashi, G. Li, and M. Takata, 2018, Mon. Not. R. Astron. Soc. 477, 2183.

Saio, H., and C. S. Jeffery, 2019, Mon. Not. R. Astron. Soc. 482, 758.

Saio, H., D. W. Kurtz, S. J. Murphy, V. L. Antoci, and U. Lee, 2018, Mon. Not. R. Astron. Soc. 474, 2774.

Saio, H., D. W. Kurtz, M. Takata, H. Shibahashi, S. J. Murphy, T. Sekii, and T. R. Bedding, 2015, Mon. Not. R. Astron. Soc. 447, 3264.

Saio, H., et al., 2006, Astrophys. J. 650, 1111.

Saio, H., et al., 2007, Astrophys. J. 654, 544.

Salaris, M., and S. Cassisi, 2017, R. Soc. Open Sci. 4, 170192.

Sana, H., S. E. de Mink, A. de Koter, N. Langer, C. J. Evans, M. Gieles, E. Gosset, R. G. Izzard, J. B. Le Bouquin, and F. R. N. Schneider, 2012, Science 337, 444.

Santos, A. R. G., et al., 2019, Astrophys. J. 883, 65.

Savonije, G. J., and J. C. B. Papaloizou, 1997, Mon. Not. R. Astron. Soc. 291, 633.

Scargle, J. D., 1982, Astrophys. J. 263, 835.

Schmid, V. S., and C. Aerts, 2016, Astron. Astrophys. 592, A116.

Schofield, M., et al., 2019, Astrophys. J. Suppl. Ser. 241, 12.

Schonhut-Stasik, J., D. Huber, C. Baranec, C. Lamman, M. Salama, R. Jensen-Clem, D. A. Duev, R. Riddle, S. R. Kulkarni, and N. M. Law, 2020, Astrophys. J. 888, 34.

Schwarzenberg-Czerny, A., 1991, Mon. Not. R. Astron. Soc. 253, 198. Schwarzenberg-Czerny, A., 1998, Mon. Not. R. Astron. Soc. 301, 831. Serenelli, A., et al., 2017, Astrophys. J. Suppl. Ser. 233, 23.

Serenelli, A., et al., 2021 (to be published) [arXiv:2006.10868].

Sharma, S., et al., 2019, Mon. Not. R. Astron. Soc. 490, 5335.

Shibahashi, H., 1979, Publ. Astron. Soc. Jpn. 31, 87, http://articles .adsabs.harvard.edu/full/1979PASJ...31...87S.

Shibahashi, H., and M. Takata, 1993, Publ. Astron. Soc. Jpn. 45, 617, http://articles.adsabs.harvard.edu/full/1993PASJ...45..617S.

Silva Aguirre, V., et al., 2012, Astrophys. J. 757, 99. 
Silva Aguirre, V., et al., 2015, Mon. Not. R. Astron. Soc. 452, 2127. Silva Aguirre, V., et al., 2017, Astrophys. J. 835, 173.

Silva Aguirre, V., et al., 2018, Mon. Not. R. Astron. Soc. 475, 5487. Silva Aguirre, V., et al., 2020, Astron. Astrophys. 635, A164.

Skumanich, A., 1972, Astrophys. J. 171, 565.

Smeyers, P., and A. Moya, 2007, Astron. Astrophys. 465, 509.

Smeyers, P., and T. Van Hoolst, 2010, Linear Isentropic Oscillations of Stars: Theoretical Foundations, Astrophysics and Space Science Library Vol. 371 (Springer-Verlag, Berlin), https://doi.org/10.1007/ 978-3-642-13030-4.

Sódor, Á., et al., 2014, Astron. Astrophys. 568, A106.

Sonoi, T., R. Samadi, K. Belkacem, H. G. Ludwig, E. Caffau, and B. Mosser, 2015, Astron. Astrophys. 583, A112.

Soszyński, I., et al., 2016, Acta Astron. 66, 131, http://acta.astrouw .edu.pl/Vol66/n2/a_66_2_1.html.

Soufi, F., M. J. Goupil, and W. A. Dziembowski, 1998, Astron. Astrophys. 334, 911, http://articles.adsabs.harvard.edu/full/1998A $\% 26 \mathrm{~A} . . .334 . .911 \mathrm{~S}$.

Southworth, J., D. M. Bowman, A. Tkachenko, and K. Pavlovski, 2020, Mon. Not. R. Astron. Soc. 497, L19.

Southworth, J., H. Bruntt, and D. L. Buzasi, 2007, Astron. Astrophys. 467, 1215.

Stello, D., H. Bruntt, H. Preston, and D. Buzasi, 2008, Astrophys. J. Lett. 674, L53.

Stello, D., M. Cantiello, J. Fuller, D. Huber, R. A. García, T. R. Bedding, L. Bildsten, and V. Silva Aguirre, 2016, Nature (London) 529, 364.

Stello, D., D. L. Compton, T. R. Bedding, J. Christensen-Dalsgaard, L. L. Kiss, H. Kjeldsen, B. Bellamy, R. A. García, and S. Mathur, 2014, Astrophys. J. Lett. 788, L10.

Stello, D., et al., 2015, Astrophys. J. Lett. 809, L3.

Stello, D., et al., 2017, Astrophys. J. 835, 83.

Suárez, J. C., M. J. Goupil, and P. Morel, 2006, Astron. Astrophys. 449, 673.

Suárez, J. C., M. J. Goupil, D. R. Reese, R. Samadi, F. Lignières, M. Rieutord, and J. Lochard, 2010, Astrophys. J. 721, 537.

Sundqvist, J. O., V. Petit, S. P. Owocki, G. A. Wade, and J. Puls (MiMeS Collaboration), 2013, Mon. Not. R. Astron. Soc. 433, 2497.

Szewczuk, W., and J. Daszyńska-Daszkiewicz, 2017, Mon. Not. R. Astron. Soc. 469, 13.

Szewczuk, W., and J. Daszyńska-Daszkiewicz, 2018, Mon. Not. R. Astron. Soc. 478, 2243.

Takata, M., 2012, Publ. Astron. Soc. Jpn. 64, 66.

Takata, M., 2016a, Publ. Astron. Soc. Jpn. 68, 109.

Takata, M., 2016b, Publ. Astron. Soc. Jpn. 68, 91.

Takata, M., R. M. Ouazzani, H. Saio, S. Christophe, J. Ballot, V. Antoci, S. J. A. J. Salmon, and K. Hijikawa, 2020, Astron. Astrophys. 635, A106.

Talon, S., and C. Charbonnel, 2008, Astron. Astrophys. 482, 597.

Tassoul, M., 1980, Astrophys. J. Suppl. Ser. 43, 469.

Tassoul, M., 1990, Astrophys. J. 358, 313.

Tayar, J., P. G. Beck, M. H. Pinsonneault, R. A. García, and S. Mathur, 2019, Astrophys. J. 887, 203.

Telting, J. H., et al., 2012, Astron. Astrophys. 544, A1.

Terquem, C., J. C. B. Papaloizou, R. P. Nelson, and D. N. C. Lin, 1998, Astrophys. J. 502, 788.

Théado, S., G. Alecian, F. LeBlanc, and S. Vauclair, 2012, Astron. Astrophys. 546, A100.

Themeß1, N., et al., 2018, Mon. Not. R. Astron. Soc. 478, 4669.

Thompson, S. E., et al., 2012, Astrophys. J. 753, 86.

Thoul, A. A., J. N. Bahcall, and A. Loeb, 1994, Astrophys. J. 421, 828.
Timmes, F. X., R. H. D. Townsend, E. B. Bauer, A. Thoul, C. E. Fields, and W. M. Wolf, 2018, Astrophys. J. Lett. 867, L30.

Tkachenko, A., K. Pavlovski, C. Johnston, M. G. Pedersen, M. Michielsen, D. M. Bowman, J. Southworth, V. Tsymbal, and C. Aerts, 2020, Astron. Astrophys. 637, A60.

Tkachenko, A., et al., 2014, Mon. Not. R. Astron. Soc. 438, 3093.

Tong, V., and R. García, 2015, Extraterrestrial Seismology (Cambridge University Press, Cambridge, England).

Torres, G., J. Andersen, and A. Giménez, 2010, Astron. Astrophys. Rev. 18, 67.

Townsend, R. H. D., 2003a, Mon. Not. R. Astron. Soc. 343, 125.

Townsend, R. H. D., 2003b, Mon. Not. R. Astron. Soc. 340, 1020.

Townsend, R. H. D., J. Goldstein, and E. G. Zweibel, 2018, Mon. Not. R. Astron. Soc. 475, 879.

Townsend, R. H. D., and S. A. Teitler, 2013, Mon. Not. R. Astron. Soc. 435, 3406.

Trampedach, R., M. J. Aarslev, G. Houdek, R. Collet, J. ChristensenDalsgaard, R. F. Stein, and M. Asplund, 2017, Mon. Not. R. Astron. Soc. 466, L43.

Triana, S. A., E. Moravveji, P. I. Pápics, C. Aerts, S. D. Kawaler, and J. Christensen-Dalsgaard, 2015, Astrophys. J. 810, 16.

Trimble, V., and P. J. T. Leonard, 1996, Publ. Astron. Soc. Pac. 108, 8. Turcotte, S., J. Richer, and G. Michaud, 1998, Astrophys. J. 504, 559.

Unno, W., Y. Osaki, H. Ando, H. Saio, and H. Shibahashi, 1989, 2nd ed., Nonradial Oscillations of Stars (University of Tokyo Press, Tokyo).

Uzundag, M., A. S. Baran, R. H. Østensen, M. D. Reed, J. H. Telting, and B. K. Quick, 2017, Mon. Not. R. Astron. Soc. 472, 700.

Van Beeck, J., V. Prat, T. Van Reeth, S. Mathis, D. M. Bowman, C. Neiner, and C. Aerts, 2020, Astron. Astrophys. 638, A149.

VandenBerg, D. A., O. Richard, G. Michaud, and J. Richer, 2002, Astrophys. J. 571, 487.

Van Eylen, V., C. Arentoft, M. S. Lundkvist, H. Kjeldsen, J. E. Owen, B. J. Fulton, E. Petigura, and I. Snellen, 2018, Mon. Not. R. Astron. Soc. 479, 4786.

Van Eylen, V., et al., 2014, Astrophys. J. 782, 14.

Van Grootel, V., S. Charpinet, P. Brassard, G. Fontaine, and E. M. Green, 2013, Astron. Astrophys. 553, A97.

Van Grootel, V., S. Charpinet, G. Fontaine, E. M. Green, and P. Brassard, 2010, Astron. Astrophys. 524, A63.

Van Grootel, V., et al., 2010, Astrophys. J. Lett. 718, L97.

Van Hoolst, T., 1994, Astron. Astrophys. 292, 471, http://articles .adsabs.harvard.edu/full/1994A\%26A...292..471V.

Van Reeth, T., A. Tkachenko, and C. Aerts, 2016, Astron. Astrophys. 593, A120.

Van Reeth, T., et al., 2015, Astrophys. J. Suppl. Ser. 218, 27.

Van Reeth, T., et al., 2018, Astron. Astrophys. 618, A24.

Van Saders, J. L., T. Ceillier, T. S. Metcalfe, V. Silva Aguirre, M. H. Pinsonneault, R. A. García, S. Mathur, and G. R. Davies, 2016, Nature (London) 529, 181.

Verma, K., H. M. Antia, S. Basu, and A. Mazumdar, 2014, Astrophys. J. 794, 114.

Verma, K., K. Raodeo, H. M. Antia, A. Mazumdar, S. Basu, M. N. Lund, and V. Silva Aguirre, 2017, Astrophys. J. 837, 47.

Verma, K., K. Raodeo, S. Basu, V. Silva Aguirre, A. Mazumdar, J. R. Mosumgaard, M. N. Lund, and P. Ranadive, 2019, Mon. Not. R. Astron. Soc. 483, 4678.

Verma, K., and V. Silva Aguirre, 2019, Mon. Not. R. Astron. Soc. 489, 1850.

Viallet, M., C. Meakin, D. Arnett, and M. Mocák, 2013, Astrophys. J. 769, 1.

Viallet, M., C. Meakin, V. Prat, and D. Arnett, 2015, Astron. Astrophys. 580, A61. 
Viani, L. S., S. Basu, M. Joel Ong J., A. Bonaca, and W. J. Chaplin, 2018, Astrophys. J. 858, 28.

von Zeipel, H., 1924, Mon. Not. R. Astron. Soc. 84, 665.

Vrard, M., T. Kallinger, B. Mosser, C. Barban, F. Baudin, K. Belkacem, and M. S. Cunha, 2018, Astron. Astrophys. 616, A94.

Wade, G. A., et al., 2016, Mon. Not. R. Astron. Soc. 456, 2.

Walker, G., et al., 2003, Publ. Astron. Soc. Pac. 115, 1023.

Walker, G. A. H., et al., 2005a, Astrophys. J. Lett. 635, L77.

Walker, G. A. H., et al., 2005b, Astrophys. J. Lett. 623, L145.

Weinberg, N. N., and P. Arras, 2019, Astrophys. J. 873, 67.

Weiss, W. W., et al., 2014, Publ. Astron. Soc. Pac. 126, 573.

Welsh, W. F., et al., 2011, Astrophys. J. Suppl. Ser. 197, 4.

White, T. R., et al., 2013, Mon. Not. R. Astron. Soc. 433, 1262.

White, T. R., et al., 2018, Mon. Not. R. Astron. Soc. 477, 4403.

Willems, B., 2003, Mon. Not. R. Astron. Soc. 346, 968.

Winget, D. E., et al., 1991, Astrophys. J. 378, 326.

Winget, D. E., et al., 1994, Astrophys. J. 430, 839.

Witte, M. G., and G. J. Savonije, 1999, Astron. Astrophys. 341, 842, http://articles.adsabs.harvard.edu/full/1999A\%26A...341..842W.

Wu, T., and Y. Li, 2019, Astrophys. J. 881, 86.

Wu, Y., 2001, Mon. Not. R. Astron. Soc. 323, 248.

Yu, J., T. R. Bedding, D. Stello, D. Huber, D. L. Compton, L. Gizon, and S. Hekker, 2020, Mon. Not. R. Astron. Soc. 493, 1388.
Yu, J., D. Huber, T. R. Bedding, D. Stello, M. Hon, S. J. Murphy, and S. Khanna, 2018, Astrophys. J. Suppl. Ser. 236, 42.

Zahn, J.-P., 1975, Astron. Astrophys. 41, 329, http://articles.adsabs .harvard.edu/full/1975A\%26A....41..329Z.

Zahn, J.-P., 1991, Astron. Astrophys. 252, 179, http://articles.adsabs .harvard.edu/full/1991A\%26A...252..179Z.

Zahn, J.-P., 1992, Astron. Astrophys. 265, 115, http://articles.adsabs .harvard.edu/full/1992A\%26A...265..115Z.

Zerbi, F. M, et al., 1999, Mon. Not. R. Astron. Soc. 303, 275.

Zhou, Y., M. Asplund, and R. Collet, 2019, Astrophys. J. 880, 13.

Zinn, J. C., M. H. Pinsonneault, D. Huber, D. Stello, K. Stassun, and A. Serenelli, 2019, Astrophys. J. 885, 166.

Zong, W., S. Charpinet, and G. Vauclair, 2016, Astron. Astrophys. 594, A46.

Zong, W., S. Charpinet, G. Vauclair, N. Giammichele, and V. Van Grootel, 2016, Astron. Astrophys. 585, A22.

Zwintz, K., 2008, Astrophys. J. 673, 1088.

Zwintz, K., W. W. Weiss, R. Kuschnig, R. Gruber, S. Frandsen, R. Gray, and H. Jenkner, 2000, Astron. Astrophys. Suppl. Ser. 145, 481.

Zwintz, K., et al., 2009, Astron. Astrophys. 502, 239.

Zwintz, K., et al., 2014, Science 345, 550. 